\title{
A method for the conceptual design of hybrid electric air-
}

craft

J. Zamboni

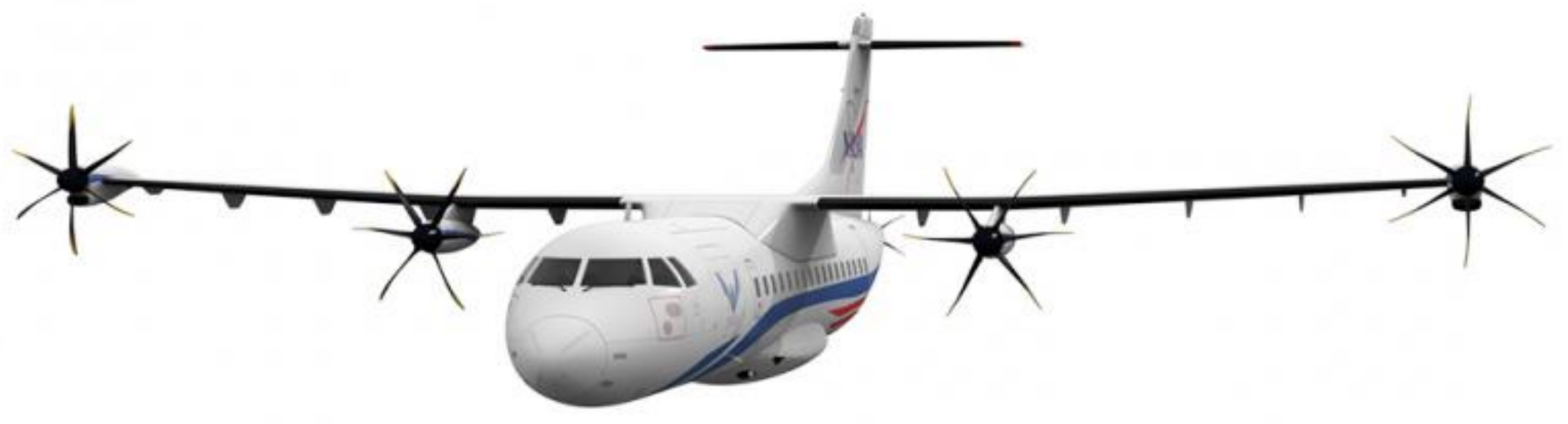





\section{A METHOD FOR THE CONCEPTUAL DESIGN OF HYBRID ELECTRIC AIRCRAFT}

by

\section{J. Zamboni}

in partial fulfillment of the requirements for the degree of

\section{Master of Science}

in Aerospace Engineering

at the Delft University of Technology,

to be defended publicly on Thursday August 23, 2018 at 1:00 PM.

$\begin{array}{lll}\text { Supervisor: } & \text { Dr. ir. R. Vos, } & \text { TU Delft } \\ \text { Thesis committee: } & \text { Prof. dr. ir. L. L. M. Veldhuis, } & \text { TU Delft } \\ & \text { Ir. P. C. Roling, } & \text { TU Delft } \\ & \text { Ir. R. de Vries, } & \text { TU Delft }\end{array}$

Cover picture: the PEGASUS hybrid electric aircraft concept by K.R. Antcliff and F.M. Capristan[1].

An electronic version of this thesis is available at http://repository.tudelft.nl/.

\section{TUDelft}





\section{ACKNOWLEDGEMENTS}

This thesis marks the conclusion of an intense yet exciting year of work characterized by many challenges but also much satisfaction in learning and growing as an engineer and a person. Looking back at the first struggles in learning the intricacies of a complex preliminary design software, at the long days spent debugging and reiterating the ideas that I wanted to implement, it seems now that it was worth it as along the way I deepened my knowledge in a discipline I love and I had the opportunity to share these moments with wonderful people. Without them I would have not been able to complete this project.

I would like to extend a special thanks to my supervisor, Roelof, as he believed in my capabilities and guided me throughout my permanence at TU Delft. I particularly enjoyed the discussions about new developments and projects in the aircraft design world as the passion he puts in his work transpires in these moments. Another thanks goes to Reynard and Malcom, that were always available to help me with suggestions and by sharing their findings whenever I was struggling with an obstacle. The already difficult process of learning to use and modify a full-fledged design application would have been impossible without the help of the people of Pace. A special thanks goes to Mathias, Carlos and Alexander that took time off their work to walk me through the new software version when I visited them in Berlin. I really enjoyed the Pace conference days and it was exciting to see so many people interested in the developments of hybrid electric aircraft.

The largest thank you goes to my friends and family as they are the people that supported me the most in the difficult times and cheered me on when I needed it. In particular, I cannot thank enough Andrea and Francesco with whom I shared these years at TU Delft, working together on many challenging projects. I value the every-day discussions about history, aviation, the future or whatever was coming to our minds those days. A big hug goes to my sisters that brought me a lot of joy every-time they came to visit, to my mother that made sure that I was eating enough and enjoying life, and to my father that supported and advised me along the way. Finally, to my fiancée, Xiaolu, I want to dedicate this work as you followed me to Delft supporting my decision of moving abroad to expand my horizons even if it meant leaving friends and work behind. I love every moment that I spend with you and I would not be surprised if, after all the times you had to listen to my blabbering about my work, you would be ready to also start a career in aerospace engineering.

J. Zamboni

Delft, August 2018 



\section{SUMMARY}

As the aviation sector keeps expanding, a growing interest in technologies that can reduce the dependency from non-renewable energy sources, both for economic and environmental reasons, has led researchers to investigate the opportunities offered by the electrification of flight. It is theorized that the use of electrically powered machines as means of propulsion could reduce the emission impact of future aircraft through a combination of a higher energy conversion efficiency and synergies with the overall aircraft system. However, fully electric designs are not viable in the near and medium term as the performance characteristics of the electric devices are still not comparable with the achievements in terms of high specific weights of modern fuel-burning propulsive system.

A proposed solution while the technology maturity level of the electric devices advances, is to electrify only a fraction of the aircraft system. The use of two energy sources in the propulsive system opens the design space and allows for the experimentation with novel aircraft configurations that could lead to interesting fuel and energy consumption reductions. However, established methods for aircraft design become obsolete as the complex configurations and control strategies cannot be modelled. Without fully taking advantage of the degrees of freedom introduced by the hybridization of the propulsive system, it is expected that little improvements will be seen as the resulting designs would not differ much from the modern aircraft.

The objective of this thesis is to develop a conceptual design procedure that can be applied to size and analyse any hybrid electric architecture using electro-chemical batteries as the electric energy source. A conceptual design method needs to be simple and adaptable so that a wide range of designs with morphologically different propulsive system architectures can be quickly compared and improved.

The proposed approach distils the main components required in a hybrid electric architecture into a base building block that can be used to model an arbitrarily complex system using few control parameters. The control strategy determines the power flows inside the propulsive system contemporaneously defining the type of architecture and allowing the estimation of power and energy requirements. The electric and conventional devices are sized with these requirements using low order methods that can be applied to a wide range of power ratings. The impact of the hybridization is considered both in the energy consumption estimation and in the evaluation of point performance constraints.

The proposed method is validated in two steps. First, the performance estimations derived from the main components models are compared to published data or higher order method results. Discrepancies and limitations are discussed and solutions to improve the accuracy are proposed. Then, a conventional twinengine turboprop aircraft is designed and the results are compared to assess if the integrated approach that puts the constraint analysis in communication with the point mass estimations and sizing procedures leads to valid results.

The method is then used for a design space exploration exercise where three different hybrid electric powertrain architectures are studied for varying technology maturity levels and mission control strategies. The most promising propulsive system operational strategy is then selected for each design and studied in more depth. The sensitivity of each aircraft to the main design parameters is then assessed in the final study.

The results indicate that the electrification of the propulsive system can lead to fuel and energy consumption reductions if the battery specific energy improves beyond $500 \mathrm{Wh} / \mathrm{kg}$, a value already double of what is possible with today's technologies. The improvement of the other devices such as electric motors and electric power distribution sub-system is less critical but still necessary if large fuel load reductions are to be accomplished. Finally, the full potential of the hybrid electric powertrain can be achieved only by taking advantage of synergies with the other aircraft disciplines and by carefully selecting an appropriate control strategy. 



\section{Contents}

List of Figures $\quad$ ix

List of Tables $\quad$ xi

Nomenclature $\quad$ xiii

List of Latin symbols ． . . . . . . . . . . . . . . . . . . . . . . . . . xiii

List of Greek symbols . . . . . . . . . . . . . . . . . . . . . xiv

List of subscripts . . . . . . . . . . . . . . . . . . . . . $\mathrm{xv}$

List of acronyms . . . . . . . . . . . . . . . . . . . . . xvi

1 Introduction 1

1.1 Problem definition . . . . . . . . . . . . . . . . . . . . . . 1

1.2 Research objective and approach . . . . . . . . . . . . . . . . . . . . . . . . . . . . . . . . . . . . .

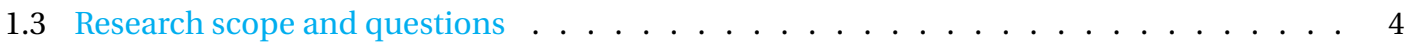

1.4 Documentstructure ......................... . . 4

2 Background Information $\quad 7$

2.1 Electric energy sources . . . . . . . . . . . . . . . . . . . . . . 7

2.1.1 Battery characteristics . . . . . . . . . . . . . . 7

2.1 .2 Lithium ion . . . . . . . . . . . . . . . . . . . . 8

2.1 .3 Lithium Sulphur . . . . . . . . . . . . . . . . . . . 9

2.1.4 Lithium oxygen . . . . . . . . . . . . . . . . . . . . 9

2.1.5 Batteries technology 2035 expectations . . . . . . . . . . . . . 11

2.2 Electrical energy distribution and conversion. . . . . . . . . . . . . . . . 11

2.2.1 Cryogenic vs non-cryogenic power distribution . . . . . . . . . . . . . 12

2.2 .2 Power distribution . . . . . . . . . . . . . . . . . . . . . 14

2.2 .3 Power modules. . . . . . . . . . . . . . . . . . . . . 15

2.3 Motor and generator technology . . . . . . . . . . . . . . . . . . 16

2.3.1 Non-cryogenic power machines . . . . . . . . . . . . . . . . 17

2.3.2 Cryogenic power machines . . . . . . . . . . . . . 18

3 Qualitative assessment of HEP configurations 19

3.1 Degree of Hybridization. . . . . . . . . . . . . . . . . . . . 19

3.2 Series and turbo-electric configuration . . . . . . . . . . . . . . . . 21

3.3 Parallel configuration . . . . . . . . . . . . . . . . . . . . . 22

3.4 Series-Parallel configuration . . . . . . . . . . . . . . . . . . . . . . . . . . . . . . . . . . . . . 23

3.5 Partial turbo electric configuration . . . . . . . . . . . . . . . . . . . . . . . . . . . . . . . . . . .

3.6 Qualitative comparison . . . . . . . . . . . . . . . . . . 24

4 Methodology 27

4.1 Propulsive Power Unit and control parameters definition. . . . . . . . . . . . . . . . 28

4.1.1 Propulsive Power Unit concept. . . . . . . . . . . . . . . . . . 29

4.1 .2 Control parameters . . . . . . . . . . . . . . . . . . 29

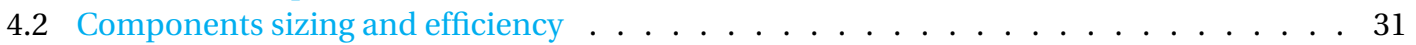

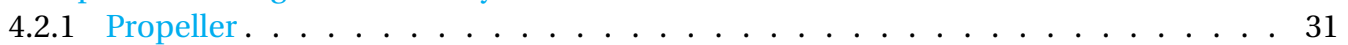

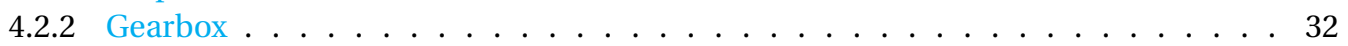

4.2 .3 Turbo-shaft engine. . . . . . . . . . . . . . . . . 33

4.2.4 Electric motor and generator. . . . . . . . . . . . . . . . . 33

4.2.5 High-power cabling system . . . . . . . . . . . . . . . . . 37

4.2.6 Electric Power Management and Distribution sub-system . . . . . . . . . . . . . 39

4.2 .7 Battery. . . . . . . . . . . . . . . . . . 39

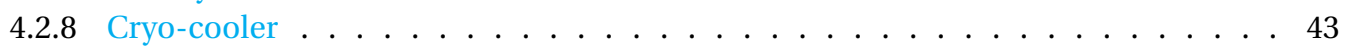




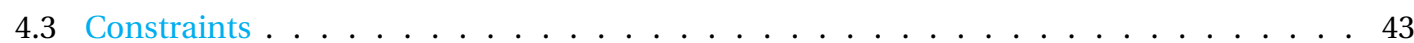

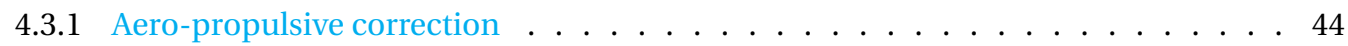

4.3.2 Propulsion efficiency correction . . . . . . . . . . . . . . . . . . . . . . . . . . . . . . 45

4.3.3 Power-lapse correction. . . . . . . . . . . . . . . . . . . . . . . . . . . . . . . . 45

4.3.4 One Engine Inoperative correction. . . . . . . . . . . . . . . . . . 47

4.4 Point performance analysis: HEP system analysis. . . . . . . . . . . . . . . . . . . . . . . . . . . . . . 48

4.4.1 Propulsive power evaluation . . . . . . . . . . . . . . . . . . . . . . . . . . . 48

4.4.2 Power-paths computations .................... 50

4.4.3 Energy consumption estimation . . . . . . . . . . . . . . 51

4.5 Hybrid electric architecture key performance indicators . . . . . . . . . . . . 51

4.5.1 Hybridization and efficiency. . . . . . . . . . . . . . . . 51

4.5 .2 Emissions . . . . . . . . . . . . . . . . . . 52

5 Validation $\quad \mathbf{5 5}$

5.1 Components validation . . . . . . . . . . . . . . . . . . . . 55

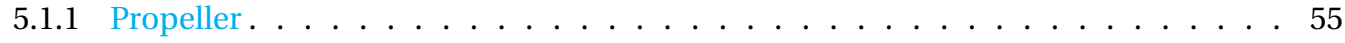

5.1 .2 Electric motor . . . . . . . . . . . . . . . . . . 57

5.1 .3 Cabling system. . . . . . . . . . . . . . . . . . . . 58

5.1 .4 Electrochemical Cell . . . . . . . . . . . . . . . . . . 59

5.2 Baseline design validation . . . . . . . . . . . . . . . . . . . 6 60

5.2 .1 Point performance constraints . . . . . . . . . . . . 6 61

5.2 .2 Mission requirements . . . . . . . . . . . . . . . . . 62

5.2 .3 Baseline results . . . . . . . . . . . . . . . . . . . 62

6 Results $\quad 65$

6.1 Hybrid Electric Powertrain architectures . . . . . . . . . . . . . . . . 65

6.1 .1 Parallel architecture . . . . . . . . . . . . . . . . 65

6.1.2 Parallel/series hybrid architecture . . . . . . . . . . . . . . 66

6.1.3 Distributed series architecture . . . . . . . . . . . . . . . . 67

6.2 Design space exploration . . . . . . . . . . . . . . . . . . . 69

6.2.1 Technology maturity level assumptions . . . . . . . . . . . . . . . . 69

6.2.2 Maximum take-off mass changes . . . . . . . . . . . . . . . . 70

6.2 .3 Mission fuel and total energy . . . . . . . . . . . . . . . . . 71

6.2.4 Overall efficiency, ESAR and well-to-propeller emissions . . . . . . . . . . 73

6.3 Hybrid Electric Aircraft analysis . . . . . . . . . . . . . . . . . . . . . 75

6.3.1 HEA constraint analysis . . . . . . . . . . . . . . . . 76

6.3 .2 Point mass analysis . . . . . . . . . . . . . . . . . . 78

6.3 .3 Resulting designs comparison . . . . . . . . . . . . . 85

6.4 Sensitivity analysis . . . . . . . . . . . . . . . . . . . . 88

7 Conclusions \& recommendation $\quad 93$

7.1 Conclusions. . . . . . . . . . . . . . . . . . . . . . 93

7.2 Limitations and recommendation . . . . . . . . . . . . . . . . . 95

$\begin{array}{ll}\text { Bibliography } & 97\end{array}$

$\begin{array}{lr}\text { A Conservative and HTS assumptions } & 103\end{array}$

$\begin{array}{lr}\text { B Hybrid Electric Aircraft schematics } & 111\end{array}$ 


\section{LIST OF FIGURES}

1.1 ACARE emissions reduction and system efficiencies goals $\ldots \ldots \ldots \ldots \ldots \ldots$

1.2 Examples of Hybrid Electric Aircraft designs . . . . . . . . . . . . . . . . . 2

2.1 Critical temperature of superconducting materials . . . . . . . . . . . . . . . . . 12

2.2 Superconducting tape and complete cable structure . . . . . . . . . . . . . . . . . 14

2.3 Aircraft megawatt generator research, development and vision . . . . . . . . . . . . . . 17

3.1 Degree of Energy and Power hybridization to describe HEP . . . . . . . . . . . . . . . . . 20

3.2 Universally electric architecture . . . . . . . . . . . . . . . . . . . 20

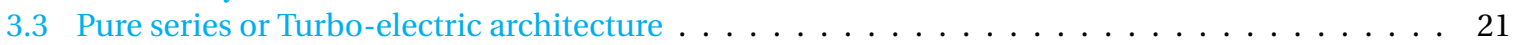

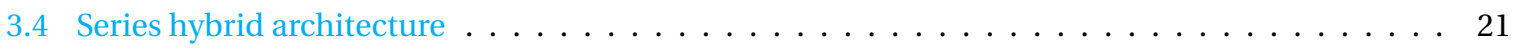

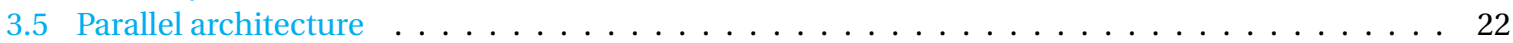

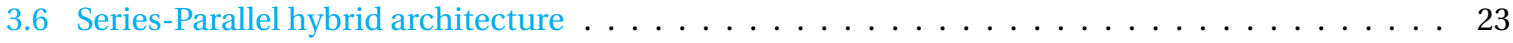

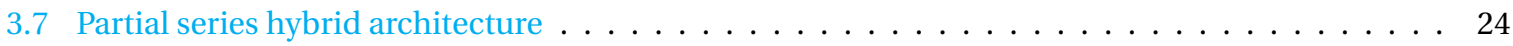

4.1 Flow chart of the proposed methodology for hybrid electric aircraft sizing. . . . . . . . . . . . 28

4.2 Schema of generic Propulsive Power Unit. . . . . . . . . . . . . . . . . . . . . . . 29

4.3 Propulsive power share used to differentiate between main (conventional) and auxiliary (electric) PPUs in a series-parallel architecture . . . . . . . . . . . . . . . . . . . . 30

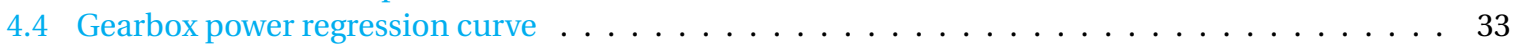

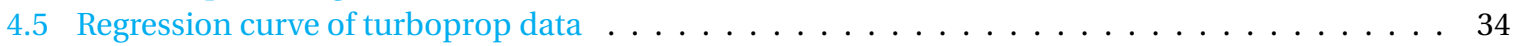

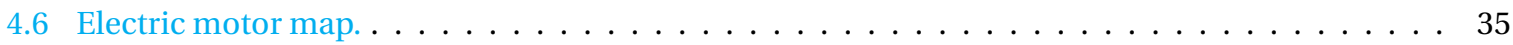

4.7 Electric motor characteristics with iso-efficiency lines. . . . . . . . . . . . . . . . 36

4.8 Cabling paths (red) along wing span connecting motor inverters (green) to the PMAD subsystem (black). . . . . . . . . . . . . . . . . . . . . . . 38

4.9 Electrochemical cell model, series and parallel battery connections. . . . . . . . . . . . . . . 40

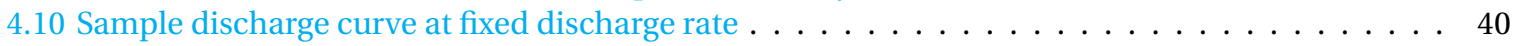

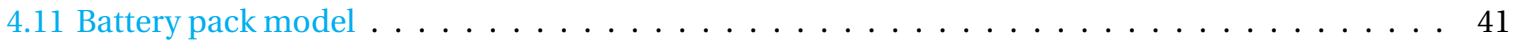

4.12 Ragone chart with battery characteristics predictions . . . . . . . . . . . . . . . 43

4.13 Changes in the power loading constraint diagram due to aero-propulsive interactions. . . . . . . 45

4.14 Changes in the power loading constraint diagram due to changes to the propulsive efficiency. $\quad$ • 46

4.15 Changes in the power loading constraint diagram due to the segment degree of electric hy-

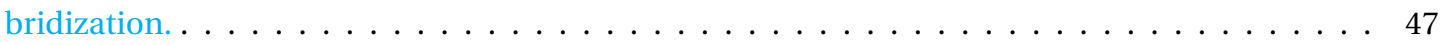

4.16 Changes in the power loading constraint diagram due to the number of propulsive devices. . . . 48

4.17 Free-body diagram of aircraft on ground. . . . . . . . . . . . . . . . . . . . 48

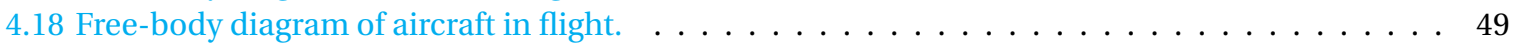

4.19 Global Warming Potentials of $\mathrm{CO}_{2}, \mathrm{H}_{2} \mathrm{O}$ and $\mathrm{NO}_{x} \ldots \ldots \ldots \ldots \ldots \ldots$

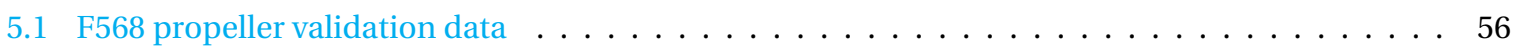

5.2 Flow chart of the proposed methodology for hybrid electric aircraft sizing. . . . . . . . . . . . 56

$5.3250 \mathrm{~kW}$ electric motor operation and efficiency charts comparison. . . . . . . . . . . . . . 57

5.4 Motor efficiency as function of shaft speed and power characteristics of $8000 \mathrm{hp}$ machine proposed for the Boeing's SUGAR Volt design . . . . . . . . . . . . . . . . . . . . . . 58

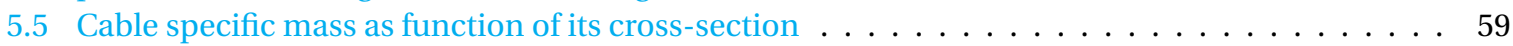

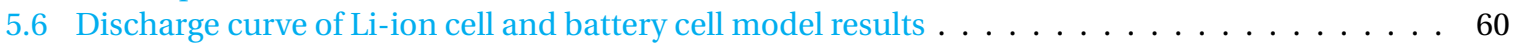

5.7 Baseline design power loading constraint diagram. . . . . . . . . . . . . . . . . . . 61

5.8 Baseline design altitude and power as function of mission time. . . . . . . . . . . . . . 63

5.9 ATR72 3-view drawing with overlay of baseline design result . . . . . . . . . . . . . . . . 64

6.1 Parallel aircraft external propulsive system configuration . . . . . . . . . . . . . . . 66 
6.2 Series/parallel aircraft external propulsive system configuration. . . . . . . . . . . . . . . . 66

6.3 Series aircraft external propulsive system configuration. . . . . . . . . . . . . . . . 68

6.4 MTOW change as a function of the control parameters for three HEP architectures and opti-

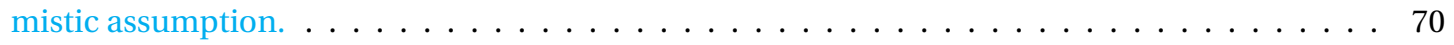

6.5 Response surface of the battery utilization as function of the control parameters. . . . . . . . . 71

6.6 Design space results for fuel mass as function of the control parameters. . . . . . . . . . . . 72

6.7 Total energy response surface for the three HEP architectures. . . . . . . . . . . . . . . . 72

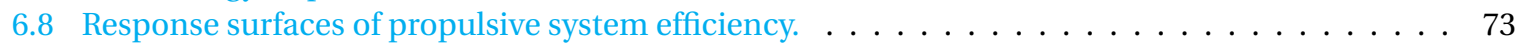

6.9 Energy Specific Air Range response surfaces. . . . . . . . . . . . . . . . . . . . . . . . . . . . . . . . . . . . . . . .

6.10 Emission $\mathrm{CO}_{2}$ equivalent impact response surfaces. . . . . . . . . . . . . . . . . 75

6.11 Power shaving control approaches with fully conventional cruise (up) and hybrid cruise (bottom). 76

6.12 Parallel architecture design constraint diagram with iso-mass and iso-energy curves. . . . . . . 77

6.13 Series/parallel architecture design constraint diagram with iso-mass and iso-energy curves. . . 78

6.14 Distributed series architecture design constraint diagram with iso-mass and iso-energy curves. 78

6.15 Power usage and mass change along mission for parallel HEP design. . . . . . . . . . . . . . 79

6.16 Electric motor map with operational requirement overlay for parallel HEP. . . . . . . . . . . . . 79

6.17 System power and battery discharge for parallel HEP. . . . . . . . . . . . . . . . . . . 81

6.18 Power usage and mass change along mission for series / parallel HEP design. . . . . . . . . . . . 82

6.19 Electric motor map with operational requirement overlay for series/parallel HEP. . . . . . . . . 82

6.20 System power and battery discharge for series/parallel HEP. . . . . . . . . . . . . . . . . . 83

6.21 Power usage and mass change along mission for series HEP design. . . . . . . . . . . . . . . 84

6.22 Electric motor map with operational requirement overlay for series HEP. . . . . . . . . . . . . . 84

6.23 System power and battery discharge for series HEP . . . . . . . . . . . . . . . . . . 85

6.24 Resulting designs mass fractions. . . . . . . . . . . . . . . . . . . . . . 86

6.25 Sensitivity study results in terms of MTOW and total energy changes relative to baseline design. 89

6.26 Sensitivity study results in terms of MTOW and total energy changes. . . . . . . . . . . . . 91

A.1 MTOM response surface conservative assumption. . . . . . . . . . . . . . . . . . . . 103

A.2 MTOM response surface futuristic assumption. . . . . . . . . . . . . . . . . . . . 103

A.3 Battery sizing requirements response surface conservative assumption. . . . . . . . . . . . . 104

A.4 Battery sizing requirements response surface futuristic assumption. . . . . . . . . . . . . . . 104

A.5 Fuel response surface conservative assumption. . . . . . . . . . . . . . . . . . . . . . . . . . . . . . . . . . . . . . .

A.6 Fuel response surface futuristic assumption. . . . . . . . . . . . . . . . . . . . . . . . . . . . . . . . . . . . . . . . .

A.7 Total energy response surface conservative assumption. . . . . . . . . . . . . . . . . 106

A.8 Total energy response surface futuristic assumption. . . . . . . . . . . . . . . . 106

A.9 System efficiency response surface conservative assumption. . . . . . . . . . . . . . . . . 107

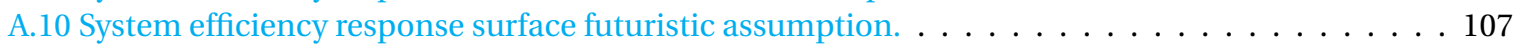

A.11 ESAR response surface conservative assumption. . . . . . . . . . . . . . . . . . . 108

A.12 ESAR response surface futuristic assumption. . . . . . . . . . . . . . . . . 108

A.13 Emissions response surface conservative assumption. . . . . . . . . . . . . . . . . . . . 109

A.14 Emissions response surface futuristic assumption. . . . . . . . . . . . . . . . . . . . . 109

B.1 Parallel architecture schematic. . . . . . . . . . . . . . . . . . . . . . . . . . . . 112

B.2 Series/parallel architecture schematic. . . . . . . . . . . . . . . . . . . 113

B.3 Series architecture schematic. . . . . . . . . . . . . . . . . . . . . . . . . 114 


\section{LIST OF TABLES}

2.1 Projected performance ranges of lithium-based batteries at cell level for the year 2035 . . . . 11

3.1 Summary of advantages and disadvantages of HEP configurations . . . . . . . . . . . . . 25

4.1 Material characteristics of conventional cables. . . . . . . . . . . . . . . . . . 38

4.2 Indicative emission indices for modern gas-turbine engine . . . . . . . . . . . . 53

5.1 Harmonic mission parameters for baseline design. . . . . . . . . . . . . . . . . . . 62

5.2 Reference and baseline conventional design. . . . . . . . . . . . . . . . . 63

6.1 Technology level assumptions for the design space exploration. . . . . . . . . . . . . . . . 69

6.2 Summary and comparison with respect to the baseline design of the three HEP analysed. . . . 87

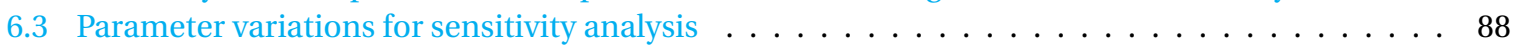





\begin{tabular}{|c|c|c|c|}
\hline $\mathrm{a}$ & $=$ & Sound speed & {$[\mathrm{m} / \mathrm{s}]$} \\
\hline A & $=$ & Area/Section & {$\left[m^{2}\right]$} \\
\hline $\mathrm{C}$ & $=$ & Capacity & {$[\mathrm{Ah}]$} \\
\hline$C_{L / D}$ & $=$ & Lift/Drag coefficient & {$[-]$} \\
\hline $\mathrm{D}$ & $=$ & Drag & {$[\mathrm{N}]$} \\
\hline $\mathrm{E}$ & $=$ & Energy & {$[\mathrm{J}]$} \\
\hline g & $=$ & Acceleration of gravity & {$\left[\mathrm{m} / \mathrm{s}^{2}\right]$} \\
\hline I & $=$ & Electric current & {$[\mathrm{A}]$} \\
\hline j & $=$ & Electric current area density & {$\left[\mathrm{A} / \mathrm{mm}^{2}\right]$} \\
\hline $\mathrm{J}$ & $=$ & Advance ratio & {$[-]$} \\
\hline k & $=$ & Correction coefficient & {$[-]$} \\
\hline 1 & $=$ & Length & {$[\mathrm{m}]$} \\
\hline $\mathrm{m}$ & $=$ & Mass & {$[\mathrm{kg}]$} \\
\hline M & $=$ & Mach & {$[-]$} \\
\hline$\dot{m}$ & $=$ & Mass flow & {$[\mathrm{kg} / \mathrm{s}]$} \\
\hline $\mathrm{n}$ & $=$ & Rotational speed & [rpm] \\
\hline $\mathrm{N}$ & $=$ & Number of & {$[-]$} \\
\hline $\mathrm{P}$ & $=$ & Power & {$[\mathrm{W}]$} \\
\hline $\mathrm{Q}$ & $=$ & Torque & {$[\mathrm{Nm}]$} \\
\hline $\mathrm{R}$ & $=$ & Electric resistance & {$[\Omega]$} \\
\hline S & $=$ & Surface & {$\left[m^{2}\right]$} \\
\hline $\mathrm{t}$ & $=$ & Time & {$[\mathrm{s}]$} \\
\hline $\mathrm{T}$ & $=$ & Thrust & {$[\mathrm{N}]$} \\
\hline $\mathrm{U}$ & $=$ & Electric voltage & {$[\mathrm{V}]$} \\
\hline $\mathrm{V}$ & $=$ & Flight speed & {$[\mathrm{m} / \mathrm{s}]$} \\
\hline $\mathrm{W}$ & $=$ & Weight & {$[\mathrm{N}]$} \\
\hline
\end{tabular}




\section{LIST OF GREEK SYMBOLS}

$\begin{array}{lll}\gamma=\text { Flight path angle } & {[\mathrm{deg}]} \\ \epsilon & =\text { Specific energy } & {[\mathrm{Wh} / \mathrm{kg}]} \\ \eta & =\text { Efficiency } & {[\%]} \\ \Theta & =\text { Propulsive power share } & {[\%]} \\ \vartheta & =\text { Relative cryogenic losses } & {[\%]} \\ \mu & =\text { Ground friction coefficient } & {[-]} \\ \rho & =\text { Air density } & {\left[\mathrm{kg} / \mathrm{m}^{3}\right]} \\ \hat{\rho}=\text { Specific power } & {[\mathrm{kW} / \mathrm{kg}]} \\ \sigma=\text { Specific resistivity } & {\left[\Omega \mathrm{mm}^{2} / \mathrm{m}\right]} \\ \Upsilon=\text { Power-lapse } & {[-]} \\ \phi & =\text { Shaft power ratio } & {[\%]} \\ \varphi=\text { Electric power ratio } & {[\%]} \\ \Phi & =\text { Supplied power ratio } & {[\%]} \\ \Phi_{b}=\text { Magnetic flux } & {[\mathrm{Wb}]} \\ \omega & =\text { Rotational speed } & {[\mathrm{rad} / \mathrm{s}]}\end{array}$




\section{LIST OF SUBSCRIPTS}

$$
\begin{aligned}
& \text { bat = Battery } \\
& \text { bemf }=\text { Back electro motive force } \\
& \text { c } \quad=\text { Cell } \\
& \mathrm{cb}=\text { Cable } \\
& \text { co }=\text { Electric conductor } \\
& \mathrm{D}=\text { Drag } \\
& \mathrm{E}=\text { Energy } \\
& \text { ec = Electric component } \\
& \text { eg }=\text { Electric generator } \\
& \text { egt }=\text { Electric generator }- \text { gasturbine system } \\
& \mathrm{em}=\text { Electric motor } \\
& \text { emg = Electric motor generator } \\
& \text { eq }=\text { Equivalent } \\
& \mathrm{f}=\text { Friction } \\
& \text { fuel }=\text { Fuel } \\
& \text { gt }=\text { Gas turbine } \\
& \mathrm{i}=\text { Internal } \\
& \text { I }=\text { Electric current } \\
& \text { iso }=\text { Isolant } \\
& \mathrm{L}=\mathrm{Lift} \\
& \lim =\text { Limit } \\
& \text { loss }=\text { Losses } \\
& \text { oc = Open-circuit } \\
& \text { ov }=\text { Overall } \\
& \mathrm{P}=\text { Propulsive power } \\
& \text { par }=\text { Parallel } \\
& \text { pr } \quad=\text { Propeller } \\
& \text { rd }=\text { Redundancy } \\
& \text { req }=\text { Required } \\
& \text { res }=\text { Result } \\
& \mathrm{s}=\text { System } \\
& \text { seg }=\text { Mission segment } \\
& \text { ser }=\text { Series } \\
& \text { sh }=\text { Shaft } \\
& \text { sheath }=\text { Cable protection } \\
& \text { th }=\text { Theoretical } \\
& \text { tip }=\text { Propeller tip } \\
& \text { Tot }=\text { Total }
\end{aligned}
$$




\section{LIST OF ACRONYMS}

\begin{tabular}{|c|c|c|}
\hline AC & $=$ & Alternating Current \\
\hline AEO & $=$ & All Engines Operative condition \\
\hline BLI & $=$ & Boundary Layer Ingestion \\
\hline CAS & $=$ & Calibrated Air Speed \\
\hline DC & $=$ & Direct Current \\
\hline DEP & $=$ & Distributed Electric Propulsion \\
\hline DoD & $=$ & Depth of Discharge \\
\hline DoH & $=$ & Degree of Hybridization \\
\hline EI & $=$ & Emission Index \\
\hline EMF & $=$ & Electro-Motive Force \\
\hline ESAR & $=$ & Energy Specific Air Range \\
\hline GWP & $=$ & Global Warming Potential \\
\hline HEA & $=$ & Hybrid Electric Aircraft \\
\hline HEP & $=$ & Hybrid Electric Powetrain \\
\hline HTS & $=$ & High Temperature Super-conductive \\
\hline KPI & $=$ & Key Performance Indicators \\
\hline Li-Air & $=$ & Lithium Air \\
\hline Li-Ion & $=$ & Lithium ion \\
\hline Li-S & $=$ & Lithium Sulphur \\
\hline MTOM/MTOW & $=$ & Maximum Take Off Mass/Weight \\
\hline OEI & $=$ & One Engine Inoperative \\
\hline OEM/OEW & $=$ & Operative Empty Mass/Weight \\
\hline PMAD & $=$ & Power Management And Distribution \\
\hline PPU & $=$ & Propulsion Power Unit \\
\hline ROD & $=$ & Rate Of Descent \\
\hline SLS & $=$ & Sea-level static condition \\
\hline SOC & $=$ & State Of Charge \\
\hline SRIA & $=$ & Strategic Research and Innovation Agenda \\
\hline TSPC & $=$ & Thrust Specific Power Consumption \\
\hline YEIS & $=$ & Year Entry Into Service \\
\hline
\end{tabular}




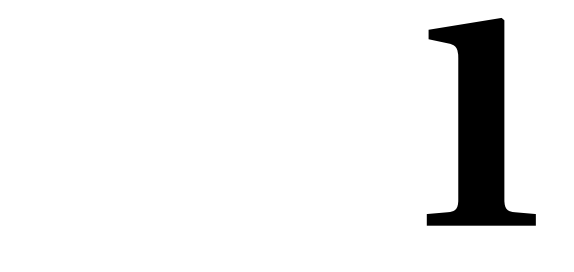

\section{INTRODUCTION}

In this introductory chapter, a quick overview of the reasons why the electrification of the aircraft propulsive system is given in section 1.1 alongside an overview of the main hybrid electric designs found in literature. The research objective, sub-goals and research approach are then explained in section 1.2. In section 1.3, the research questions are stated and the scope of this thesis is presented. The overall structure of this thesis is then given at the end of the chapter in section 1.4.

\subsection{Problem Definition}

The growing concern with global emissions and the rapidly expanding air-transport sector has brought many governmental and international agencies to elaborate aggressive emissions and noise reduction targets for the future. In Europe, the objective of Flightpath 2050 [2] is a reduction of $75 \%$ in $\mathrm{CO}_{2}$-emissions per passenger kilometre, a $90 \%$ in $N O_{x}$ emissions and a $65 \%$ in perceived noise relative to aircraft of the year 2000. On a shorter term, the Strategic Research and Innovation Agenda (SRIA) proposes the goals shown in figure 1.1 that have been recalibrated for a medium-term goal and a 2035-year entry into service aircraft.

\begin{tabular}{|c|c|c|c|c|c|c|}
\hline $\begin{array}{l}\text { Goals and } \\
\text { Key contributions }\end{array}$ & $\begin{array}{c}2000 \\
\text { (Reference) }\end{array}$ & $\begin{array}{l}2020 \\
\text { (Vision) }\end{array}$ & $\begin{array}{c}2020 \\
\text { (AGAPE) }\end{array}$ & $\begin{array}{l}2020 \\
\text { (SRIA) }\end{array}$ & $\begin{array}{c}2035 \\
\text { (SRIA) }\end{array}$ & $\begin{array}{c}2050 \\
\text { (SRIA) }\end{array}$ \\
\hline $\begin{array}{l}\mathrm{CO}_{2} \text { objective vs } 2000 \\
\text { ("HLG") }\end{array}$ & & $-50 \% * *$ & & & & $-75 \% *$ \\
\hline $\begin{array}{l}\mathrm{CO}_{2} \text { vs } 2000(\mathrm{~kg} / \mathrm{pass} \\
\mathrm{km})^{*}\end{array}$ & & $-50 \%$ & $-38 \%$ & $-43 \%$ & $-60 \%$ & $-75 \%$ \\
\hline $\begin{array}{l}\text { Airframe energy need } \\
\text { (Efficiency) }\end{array}$ & 1 & 0,75 & 0,85 & 0,8 & 0,7 & 0,32 \\
\hline $\begin{array}{l}\text { Propulsion \& Power } \\
\text { energy need (Efficiency) }\end{array}$ & 1 & 0,8 & 0,8 & 0,8 & 0,7 & \\
\hline ATM and Infrastructure & 1 & 0,88 & 0,95 & 0,93 & 0,88 & 0,88 \\
\hline $\begin{array}{l}\text { Non Infrastructure- } \\
\text { related Airlines Ops }\end{array}$ & 1 & 0.96 & 0.96 & 0.96 & 0,93 & 0,88 \\
\hline
\end{tabular}

- comparison with same transport capability aircraft and on a same mission in term on range and payload

* ACARE 2020 and ACARE 2050 High Level Goals for airframe, engine, systems and ATM/Operations

Figure 1.1: ACARE emissions reduction and system efficiencies goals [3]

It is expected that improvements in conventional technologies and architectures will not be enough to satisfy the goals set for the future. Several authors predict that even the most aggressive developments and advances in gas-turbine design and new airframe technologies would fall between 10 and $15 \%$ short of the $\mathrm{CO}_{2}$-emissions goal [4-7]. The electrification of the propulsion system is seen as one of the few approaches that will feasibly close this gap in the desired time-frame [4]. 
On the wave of the electric automotive success in lowering its environmental impact and the advances of electric storage technologies, more and more studies are looking into the benefit and feasibility of electric propulsion for the aviation sector. However, due to the lower performance parameters of electric components when compared to the conventional solutions, a fully electric aircraft is not yet seen as feasible for the civil aviation segment. Better results are instead expected using hybrid systems that combine conventional and electric propulsive technologies to decrease fuel burn and environmental impact. The expected benefits of using a hybrid electric power-train (HEP) in flight vehicles are summarised in reference [8] and reported below:

- Fewer emissions by reducing fuel burn.

- Less atmospheric heat release.

- Smaller noise impact for communities and quieter flight for passenger comfort.

- Better energy conservation and less dependence on fossil fuels.

- Better reliability by substitution of turbo-machinery with electric motors as means of propulsive power producers.

These benefits would mostly derive from the use of electric energy, that could be (partially) produced by renewable sources, of efficient and reliable electric motors that offer higher controllability ranges when compared to conventional turbine engines, and of a better integration and synergy between the propulsive system and the aircraft airframe allowed by the easier scalability of the electric components.

The possibilities offered by the hybrid and universally electric propulsive system has attracted the interest of many researchers. The introduction of this idea has also drastically opened the design space. Below a brief introduction of the most significant studies that can be found in the literature is given. It can be observed that the increased degrees of freedom in the design space has pushed the designers to experiment with new configurations that could make full use of the potential of the electrical propulsive system.

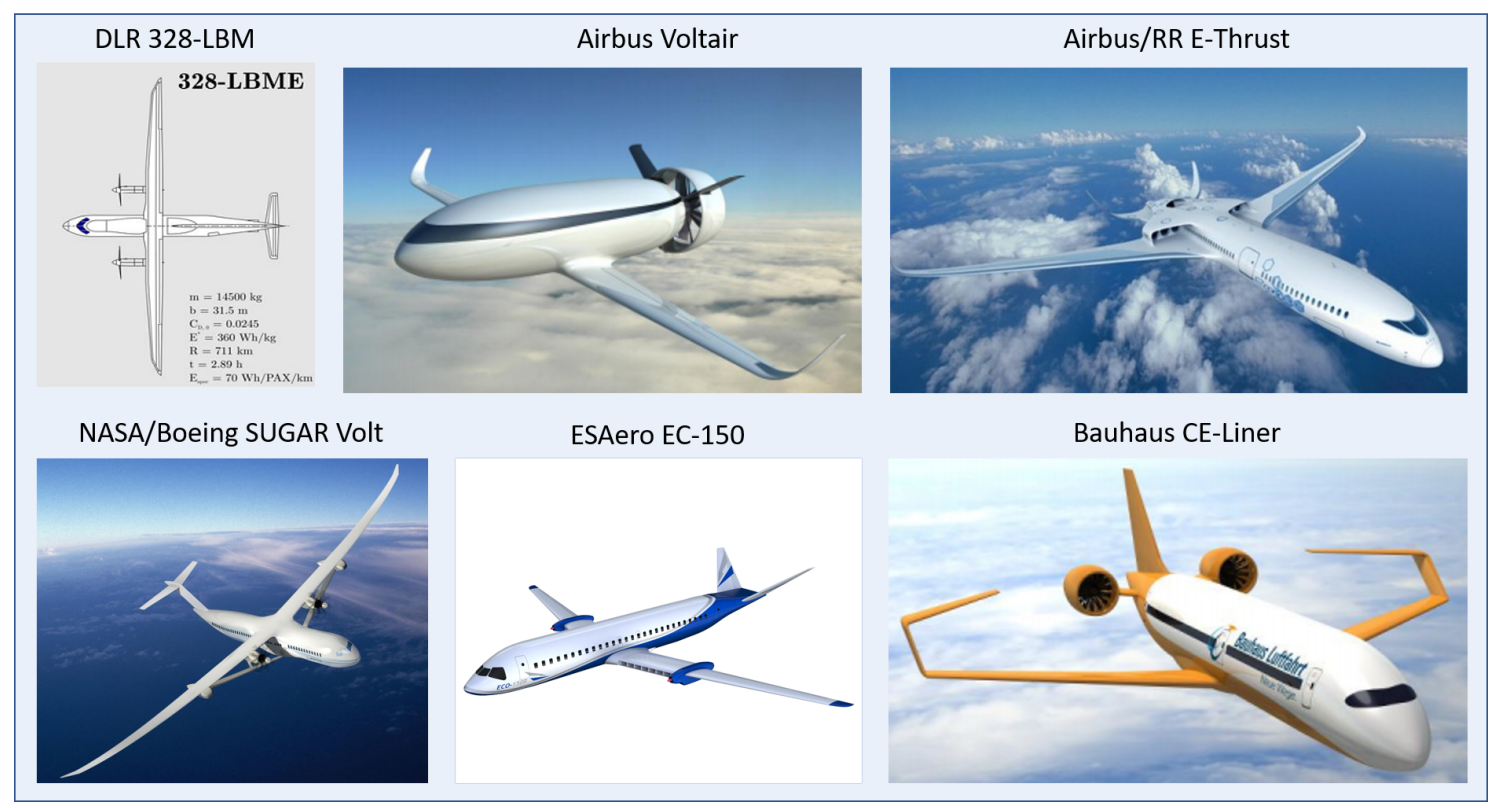

Figure 1.2: Examples of Hybrid Electric Aircraft designs

All the designs in figure 1.2 target a Year Entry Into Service (YEIS) of 2030+. From left to right, top to bottom:

- DLR 328-LBM: small electric turbo-prop that can carry around 30 passengers. It is based on a Dornier DO 328. It utilizes a universally electric configuration and very high aspect ratio wing. The $L B M E^{2}$ variant uses a $720 \mathrm{Wh} / \mathrm{kg}$ battery and offers an estimated range of $1400 \mathrm{~km}$ (without reserves) comparable to the kerosene baseline aircraft [7]. 
- Airbus Voltair: studied both as a fully-electric and hybrid electric concept, it is sized for 68 passengers, a $900 \mathrm{NM}$ range and a design speed of $500 \mathrm{~km} / \mathrm{h}$. It uses the electric motors to power a pair of shielded contra-rotating propellers mounted at the end of the fuselage to take advantage of the boundary layer ingestion to lower the overall air resistance. The authors estimate that the reference mission can be matched with $1000 \mathrm{Wh} / \mathrm{kg}$ Li-Air batteries [9].

- Airbus/RR E-Thrust: narrow body design for around 100 passengers. It uses a HEP system with 1000 $\mathrm{Wh} / \mathrm{kg}$ battery and a power turbine in the back of the fuselage. The HEP architecture is of the series kind and the thrust is produced by six electrically driven fans positioned to re-energies the aircraft wake. In addition, the fans can be used to partially re-charge the batteries during descent through fan wind milling. High Temperature Super-conductive (HTS) machines and distribution systems are enabling technologies for this design. Due to the higher technological gap, the expected YEIS is 2050 [10].

- Boeing SUGAR Volt: Hybrid Electric Aircraft design for 154 passengers. The design uses a parallel HEP configuration where electric motors supply part of the power needed by the two turboprops (or turbofan in a different variant). It is characterized by a very high aspect ratio braced wing with two large battery compartments hanging underneath it. Battery specific energy to become economically viable is stated in the range $750-1000 \mathrm{Wh} / \mathrm{kg}$ [11].

- ESAero EC-150: this aircraft is designed to fly up to 150 passengers. It employs a turbo-electric HEP meaning that the entirety of the energy is supplied by fuel-burning engines while batteries are not incorporated in the propulsive system. Two turboshafts are embedded in the wing and drive a series of small electric fans that increase the overall bypass ratio and help in filling the wake of the aircraft decreasing the wing drag. HTS technologies are needed to enable this type of aircraft [12].

- Bauhaus CE-Liner: universally electric aircraft designed for up to 189 passengers. The $1000-1500 \mathrm{Wh} / \mathrm{kg}$ batteries supply energy and power to two fuselage mounted fans driven by fully-superconducting electric motors. It uses a C-wing to reduce lift induced drag. Due to the high technology level requirements of battery and electric components, the YEIS is set as 2050[13].

This brief introduction of existing projects illustrates how vast the world of HEP can be. On top of the conventional degrees of design freedom, the aircraft designer needs now to also select electrical components for the HEP system and then estimate the best internal and external propulsive system configuration and operational strategy to maximize the benefits.

\subsection{RESEARCH OBJECTIVE AND APPROACH}

The goal of this study is to define a method for the sizing of generic hybrid electric powertrains complex enough to model all the most studied architectures found in literature and to be sensitive to top level requirements, propulsion architecture configuration and technology maturity level of its components while being simple enough to be used for extensive explorations of the design space. The added complexity and larger design space deriving from the use of a hybrid propulsion systems requires a modification of well-established design methods to fully capture its impact on the aircraft design. The co-existence of multiple energy sources and power paths disrupts commonly used sizing routines as the result is highly influenced by the relative size of the power systems and their operation along the mission flight path.

To satisfy the main objective of this research, several steps are required. Firstly, a literature study is carried out to identify the main components of hybrid electric powertrains and their performance expectations for the YEIS of 2035-2040 chosen as reference time frame for this study. Then, methods to size these components need to be implemented and tested to asses if they are suitable for the conceptual design phase. If no suitable method is found, either a lower fidelity approach is used, or a new process is proposed and validated.

A procedure to model the overall propulsive system in terms of mass and energy consumption is then developed. Attention is given to keeping a high level of abstraction so that the proposed approach can be used in the design of any hybrid electric system, no matter how complex. This is done by defining a basic building block that can be modelled into any HEP configuration using few control laws.

The models for each component and the overall sizing procedure are then implemented in a framework that permits their communication so that the entire propulsion system can be sized in an integrated approach. 
For this, the software Pacelab APD has been chosen as it offers a preliminary design environment that allows the user to implement new modules and analyse different aircraft configurations and system architectures. Before deriving conclusions from the proposed approach, the sizing process needs to be validated. This is done with experimental results or published data whenever possible or with higher fidelity methods when not.

With the validated model it is then possible to conduct investigations into the complex design space of hybrid electric aircraft. To do so, a small number of configurations is chosen, and their control strategy is used to investigate the changes in terms of Key Performance Indicators (KPI) such as the aircraft mass, fuel and energy consumption, emissions and system efficiency by comparing them to a baseline design. The results of the control strategy approach are then used to further investigate the sensitivity of the method to the technology level and mission requirements. From these studies, conclusions on the applicability of the developed method are drawn.

\subsection{RESEARCH SCOPE AND QUESTIONS}

Although the proposed method could be applied in the conceptual design of jet and propeller aircraft alike, the scope of this study is limited to turbo-prop regional aircraft. The main reason for this choice is that a preliminary background investigation indicated that the performance expectations of the electric components would lead to little to none benefits for larger aircraft in the considered time frame. The poor specific energy of the electric power sources limits the design range while constraints to the maximum power rating of the motors limits the amount of electrification that can be feasibly introduced on larger aircraft [8].

The proposed method is used to answer the following research questions:

- What are the merits of hybrid electric regional aircraft over a conventionally propelled one considering the technology level maturity of a YEIS of 2035 ?

- How do different HEP architectures compare in terms of KPI? How do they compare to a baseline design?

- What is the optimal control strategy for each HEP configuration considered?

- How are the KPI affected by different level of technology of the electric components of the propulsive system?

- Which are the critical components of the hybrid propulsive system in terms of high sensitivity to the sizing parameters variation?

- Among the studied HEP architectures, which one performs the best regarding the KPI?

- Which configuration is the most feasible if the technology level expectations are not reached?

- Which powertrain configuration is the most promising if the technology level expectations are exceeded?

\subsection{DOCUMENT STRUCTURE}

This document is structured as follows: in chapter 2 the main information regarding the electric components that are needed in an electric propulsive system are presented. Then, an overview of the possible ways in which these components can be connected is given in chapter 3 where the most common hybrid electric powertrain architectures are presented and compared qualitatively.

The methodology of the new conceptual design approach for hybrid electric aircraft is contained in chapter 4. The methods presented are then validated in chapter 5 by first validating the propulsive system components, 5.1, and then by comparing a baseline aircraft to known results from the literature research 5.2. This design is then used as a reference in the rest of the result analysis.

The results in chapter 6 are subdivided in four sections: first the HEP architectures under study and the relevant assumptions are presented in section 6.1, then a wide design space exploration is carried out to obtain insights on the best operational strategy for each hybrid electric architecture as presented in 6.2. The results 
of this design space exploration are further developed in section 6.3 to compare the HEP configurations in terms of key performance indicators and finally a sensitivity study presented in 6.4 is done to assess the most critical technologies and characteristics for the studied designs. Chapter 7 contains the conclusions of the thesis and recommendations for future developments. 



\section{2}

\section{BACKGROUND INFORMATION}

In the following chapter, the state of the art and expectations for the technologies that are needed in a hybrid electric propulsion system are identified and discussed. These technologies are grouped in energy storages (batteries), energy transmissions (cables and power modules) and energy converters (generators, motors and engines). Attention will be given in the description of the advantages and disadvantages of a given technology.

\subsection{ELECTRIC ENERGY SOURCES}

One the most important technology for the success of electric flight is a reliable energy storage that can provide the required power and energy while being light, compact and safe. There are three technologies that can somewhat offer this: electro-chemical batteries, ultra-capacitors and fuel cells.

This document will only discuss batteries as ultra-capacitors are only needed for high specific power applications but are severely lacking in terms of specific energy. Even the best estimates for the tageted YEIS put ultra-capacitors at specific energy on the level of advanced Li-Ion cells, well below the more advanced batteries such as Li-S (Lithium Sulphur) and Li-Air (Lithium Air) discussed below [14, 15].

Fuel cells, on the other hand, can be comparable to batteries in terms of performances [16] but they have critical issues when it comes to the storage of the fuel. The high specific energy of hydrogen is severely limited by its low energy density. Hydrogen based aircraft need either a large storage tank for gaseous hydrogen or a cryogenic high-pressure tank for carrying hydrogen in liquid form [4]. Both choices complicate the design of the already complex Hybrid Electric Aircraft (HEA) and therefore this energy source is omitted from this work.

\subsubsection{BATTERY CHARACTERISTICS}

When comparing batteries technology there are few parameters to take into consideration:

- Gravimetric energy density [Wh/kg]: also known as specific energy. It is defined as the amount of electrical energy stored for unit battery mass thus it is dependent on battery chemistry and packaging. It can be calculated as the product between the cell voltage [V] and the specific capacity $[\mathrm{Ah} / \mathrm{kg}]$.

- Specific Capacity [Ah/kg]: the gravimetric coulometric capacity represents the Amp-hours available when the battery discharges at a certain current, per unit of mass. Similarly, to the specific energy, limits to the discharge current are given by the cell chemistry and its weight is influenced both by the materials necessary in the electro-chemical process as well as the packaging of the cells.

- Specific Power [W/kg]: dependant on chemistry and packaging, it determines the weight required to achieve a given performance. It exists a strong trade-off between specific energy and specific power as for high discharge rates during high power demands, the battery capacity drops rapidly, significantly 
lowering the specific energy [17]. Although the maximum power that can be provided by a battery pack can be scaled by increasing the number of cells, its specific power remains virtually unchanged.

- Energy density $\left[\mathrm{Wh} / \mathrm{m}^{3}\right.$ ] or [Wh/l]: along with the energy consumption of the vehicle, it determines the volume occupied by the battery. It is function of packaging and chemistry.

- Stored Energy [J] or [Wh]: the energy stored is equal to the product of the battery voltage $\mathrm{V}$ and the capacity Q $[\mathrm{Ah}]$. The stored energy is function of how quickly a battery is charged or discharged as with increasing currents the internal losses grow.

- State of Charge (SOC) [\%]: it is defined as the ratio between the remaining capacity and the nominal capacity. It goes from $100 \%$ (fully charged) to $0 \%$ (fully discharged) but batteries usually have a practical lower SOC limit under which the cells are permanently damaged. This limit is usually around SOC $=20-30 \%$ for modern Li-Ion batteries but improvements to this value are expected as discussed in the following sections.

- Depth of Discharge (DoD) [\%]: it is the rate of discharged capacity over the nominal capacity. It is defined also as $\mathrm{DoD}=1-\mathrm{SOC}$.

- Cost (\$/Wh): depending on cost of raw materials and industrial processes required to manufacture the battery.

Based on the previous parameters, three battery technologies have been selected for further study: advanced Li-ion, Li-S and Li-air. All these technologies are based on a red-ox chemistry reaction concerning the chemical element Lithium. This is due to its high chemical potential and low weight. However, lithium high reactivity poses safety hazards and its cost could hinder the market potential on very large scales. Other promising chemistries such as molten salt and metal-air batteries have been omitted from this document because they are either less studied at the moment or the expectations for the year 2035 are lower than the lithium-based ones [18].

In the following sections a more detailed overview of the different battery technologies is given. Attention will be given to current technology, performance, safety concern, cost and prospects for the year 2035.

\subsubsection{LITHIUM ION}

In recent times, Lithium-ion has been the most widely used chemistry in batteries for electric vehicles thanks to its good specific energy and compactness [19]. A conventional Li-ion cell has a graphite anode, a cathode made of lithium metal oxide and an electrolyte made of an organic solvent and lithium salts in solution.

The maximum theoretical specific energy can be calculated based on the mass of the electrodes and their chemistry. The most commonly used cell chemistry is:

$$
L i_{x} C_{6} \rightleftharpoons \mathrm{Li}_{1-x} \mathrm{CoO}_{2}
$$

This reaction is characterized by a specific energy of $584 \mathrm{Wh} / \mathrm{kg}$ as calculated in reference [20]. However, the specific energy value is also dependant on the cell and case design. In practice, the maximum achievable gravimetric energy per cell is around $210 \mathrm{Wh} / \mathrm{kg}$ and for the battery it is around $150 \mathrm{Wh} / \mathrm{kg}$ [19, 20] while the energy density can be as high as $650 \mathrm{Wh} / 1[18,21]$.

Regarding safety, the lithium-ion batteries can prove dangerous at extreme operational conditions. In conditions such as the upper-limit of the charge process, the high local temperatures can release oxygen from the cathode. If the oxygen then reacts with the flammable organic electrolyte there is a serious risk of combustion and even explosion [21]. Possible measures to prevent the thermal runaway process are being studied, however, they negatively impact the energy performance parameters [19].

Until recently most of the work done to increase Li-ion performance has been spent on improving the casing and the fabrication processes, for example, by moving from a stainless-steel case to a lighter one made of aluminium [19]. Nowadays, the research focus is shifting to finding safer and better performing electrodes 
and electrolytes. Significant attention is being given to ionic liquids that could substitute the organic electrolyte increasing safety and reliability. Moreover, studies show that by changing the graphite anode with higher capacities materials (such as $L i_{4,4} S n$ and $L i_{4,4} S i$ ) the performance can be further improved [19, 21]. It is expected that by 2035 the lithium-ion batteries technology will have fully matured and it will reliably store between 250 and $350 \mathrm{Wh} / \mathrm{kg}$ at battery level [18, 20,21].

\subsubsection{Lithium Sulphur}

As discussed in the previous section, to increase the performances of lithium-based energy storages there is a need to experiment with new chemical combinations. To further increase the gravimetric energy, studies are being done to upgrade the cathode material composition. One of the most studied and promising one is represented by the lithium-sulphur battery characterized by the chemical reaction:

$$
S_{8}+16 L i \rightleftharpoons 8 \mathrm{Li}_{2} S
$$

This chemical reaction has a maximum specific energy of $2500 \mathrm{Wh} / \mathrm{kg}$ and an energy density of $2800 \mathrm{Wh} / \mathrm{l}$ that are almost an order of magnitude higher than the currently used in Li-ion batteries [19, 22].

In practice, gravimetric energy densities of 300-350 Wh/ kg at cell level and of 200-250 Wh/ kg at battery level are ready for commercialization $[18,23]$. In addition, a "laboratory type" battery characterized by a specific energy of $600 \mathrm{Wh} / \mathrm{kg}$ and energy density of $150 \mathrm{Wh} / \mathrm{l}$ (at cell level) is already being tested [22]. Another attractive characteristic of this technology is that sulphur is cheap and readily available thus helping in lowering the cost of the raw materials when compared to Li-ion energy storages [22].

This novel battery technology was investigated for several decades but its practical development had been hindered by several shortcomings until recently [19, 22-24], namely:

1. High solubility of poly-sulphides $L i S_{x}$ in the organic electrolyte: this causes a loss of active mass and low utilization of the cathode that negatively affects capacity decay.

2. Large volume expansion and contraction during charge/discharge cycles: this phenomenon is critical since the volume change cracks the material of the cathode disintegrating it in few cycles.

3. Low coulombic efficiency of sulphur: it affects the cell efficiency since the energy extraction is hindered and part of the sulphur is almost inaccessible.

In summary, the practical application of Li-S batteries has been kept back by very low life cycle and low efficiency that does not permit the full extraction of the chemical energy. Despite all, the interest in the technology is still important and many institutions are actively trying to solve these technical challenges. Some breakthroughs made using nano-structures in the cathode and the wrapping of sulphur in graphene structures are already proving successful in solving the challenges of volume expansion and high solubility cited above increasing both battery life (from less than 1000 to 1500 cycles) and efficiency [19, 23, 24]. Several researchers agree that the Li-S batteries is worthy of further study as life of 2500 cycles and specific energy of $600-700 \mathrm{Wh} / \mathrm{kg}$ at cell level are foreseeable in the medium and long term [18, 22].

\subsubsection{LITHIUM OXYGEN}

$\mathrm{Li}-\mathrm{O}_{2}$ battery share the same anode of Li-S batteries and they both have an active cathode that helps in increasing specific energy. This technology is characterized by the chemical reaction:

$$
2 \mathrm{Li}_{(s)}+\mathrm{O}_{2,(\mathrm{~g})} \rightleftharpoons \mathrm{Li}_{2} \mathrm{O}_{2,(s)}
$$

For this reaction to happen, the oxygen is dissolved in the electrolyte and reacts with the lithium on the surface of the porous cathode. When charging, the lithium peroxide $\mathrm{Li}_{2} \mathrm{O}_{2}$ is broken down and the reaction releases oxygen gas and plates the electrode with lithium. The specific energy and density of the reaction are the highest of the considered electric energy technologies at $3458 \mathrm{Wh} / \mathrm{kg}$ and $3445 \mathrm{Wh} / \mathrm{l}$ [25].

In practice, the battery presents still significant challenges similar to the Li-S battery. In fact, the cell has problems with safety, low charge and discharge rates, poor energy efficiency and limited life cycles. This is 
partly due to the challenge of having gaseous oxygen that need to be supplied or recirculated [20,26]. There are two ways to introduce the oxygen: harnessing it from the environment (open-cycle battery) or having it enclosed in the cell (closed-cycle battery) [25]. Both ways have their pros and cons that will be briefly described in the following sections.

\section{OPEN CYCLE}

The open-cycle architecture uses the oxygen freely available in the atmosphere. This has the advantage that part of the reactants does not need to be carried along all the time thus increasing the specific energy of the battery. However, this architecture presents also additional challenges due to the system components:

1. Separator: normal constituents of air, $\mathrm{CO}_{2}$ and $\mathrm{H}_{2} \mathrm{O}$, negatively impact the $\mathrm{Li}-\mathrm{O}_{2}$ chemistry reaction. Carbon dioxide reacts with the lithium peroxide forming $\mathrm{Li}_{2} \mathrm{CO}_{3}$ that is thermodynamically more stable, and it decompose at high voltages thus severely reducing the ability for recharge. Water vapour also critically affects the cell by spurring unwanted side reactions. It is therefore necessary to use a separator to reduce the contaminants concentration as much as possible.

2. Compressor: a compressing unit is needed to reduce the size of the absorption unit [25] and increase the air pressure at altitude.

3. Solvent addition/capture components: authors of reference [25] also suggest that the use of electrolyte solutions require that the oxygen is enriched with a solvent before entering the cell and purified from it at the outlet.

All these components add to the complexity, weight and cost of the open-cycle $\mathrm{Li}-\mathrm{O}_{2}$ battery. Moreover, the reaction has also the characteristic of gaining weight during discharge as the oxygen is bound to the lithium. The increase in weight is estimated at around $1.9 \cdot 10^{-4} \mathrm{~kg} / \mathrm{Wh}[27]$.

It is calculated that the mass increase for a closed-cycle architecture would be higher than for the open-cycle one [25]. Moreover, these calculations where performed with an auto-vehicle in mind and in a hybrid electric aircraft there would also be the choice of spilling air from the compressor of the gas-turbine engine, partly reducing the necessary components. Despite this, the use of this battery in an aviation setting must also consider the reduction of oxygen with the change in altitude, making the sizing process more complex.

Open-cycle $\mathrm{Li}-\mathrm{O}_{2}$ batteries are still in an early development phase but several analyses put their short-term specific energy for the overall system at around $450 \mathrm{Wh} / \mathrm{kg}$ and energy density at $450 \mathrm{Wh} / 1$ [18, 25]. The cell specific energy is then expected to greatly increase as the technical challenges are surmounted. Expectations of up to $1500 \mathrm{Wh} / \mathrm{kg}$ at cell level are cited in literature [27] however it is essential to consider the impact of the additional components required by this specific battery when selecting it.

\section{Closed CyCle}

The second option for oxygen introduction is to have a close-cycle architecture. This is possible as the cell would be inside a pressurized vessel containing pure oxygen [25, 27]. The vessel would naturally pressurize during the charge cycle with only small losses due to heat of the compression.

Clearly, this system does not need the complex components needed to separate air components and for the addition/removal of solvent thus making the choice cheaper and less complex. However, the technology still faces challenges such as:

1. High pressure vessel (authors suggest a 15-20 MPa vessel [25]), in which the cell stacks are installed.

2. Safety hazards given by the presence of flammable electrodes and pure oxygen in the pressurized vessel.

3. Non-optimal cooling and heating via heath sinks due to the vessel enclosure.

The expected short-term performance of the close-cycle architecture is around $260-380 \mathrm{Wh} / \mathrm{kg}$ and $410 \mathrm{Wh} / \mathrm{l}$ [18]. Cell level specific energy for the year 2035 is expected to increase up to $1200 \mathrm{Wh} / \mathrm{kg}$ [25, 27], however, to the challenges posed by the chemistry reaction and issues for its safe containment make these expectations highly uncertain. 


\subsubsection{BATTERIES TECHNOLOGY 2035 EXPECTATIONS}

Table 2.1 summarises the expectations of the three battery's technologies discussed so far. The table has been compiled mostly with projected performances found by the authors of reference [18]. However, since these projections were performed for the year 2025, part of the table has been updated with values from newer publications.

Table 2.1: Projected performance ranges of lithium-based batteries at cell level for the year 2035

\begin{tabular}{|c|c|c|c|c|c|}
\hline & Unit & Li-Ion & Li-S & $\mathrm{Li}-\mathrm{O}_{2, \text { open }}$ & $\mathrm{Li}-O_{2, \text { closed }}$ \\
\hline Specific Energy & $\mathrm{Wh} / \mathrm{kg}$ & $250-350$ & $600-700$ & 800-1500 & $600-1200$ \\
\hline Specific Power & $\mathrm{W} / \mathrm{kg}$ & $500-600$ & $350-500$ & $300-400$ & $300-400$ \\
\hline Energy Density & $\mathrm{Wh} / \mathrm{l}$ & $600-800$ & $300-350$ & $1000-1700$ & $1000-1600$ \\
\hline Charge/Discharge efficiency & $\%$ & 90-95 & $70-90$ & $60-85$ & $60-85$ \\
\hline Cycle life & \# cycles & $1000-3000$ & $1000-2500$ & $500-1000$ & $500-1000$ \\
\hline Degree of Discharge & $\%$ & $70-90$ & 90-100 & $70-90$ & $70-90$ \\
\hline Lifetime & yrs. & $7-15$ & $7-14$ & $5-10$ & $5-10$ \\
\hline Cost $(\$ 2010)$ & $\$ / \mathrm{kWh}$ & 250-350 & $250-500$ & $400-800$ & $300-700$ \\
\hline Uncertainty & - & low & medium & high & high \\
\hline
\end{tabular}

As evidenced in the table, an advanced Li-Ion battery is expected to be better in terms of specific power, efficiency, life-cycle and cost when compared to the other technologies assessed in this study. This is mostly since Li-Ion batteries are expected to be fully matured by the year 2035 where the more novel energy sources could possibly still face challenges in terms of weight optimization. However, several authors report that batteries will make HEP regional and single aisle aircraft competitive with jet-fuelled design only if they have specific energy of at least $500 \mathrm{Wh} / \mathrm{kg}$ for parallel hybrid system and of $750 \mathrm{Wh} / \mathrm{kg}$ for series and mixed hybrid systems $[4,8,28]$.

Li-ion batteries will not come anywhere close to these requirements while Li-S and Li-Air batteries could optimistically be ready for use in an aviation framework. These are also the batteries with the most uncertainty due to use of novel cathodes or gaseous reactants making the range of reported specific energy wide (600$1500 \mathrm{Wh} / \mathrm{kg}$ ). No definitive method to correlate the specific energy and power at cell level to the battery level could be found in literature. Thus, a correction factor between $70 \%$ and $80 \%$ has been derived from modern batteries to correct the battery gravimetric parameters. The other battery specifications in table 2.1 will also be considered for the design of the HEP. The necessary battery mass will be function of both the specific power and gravimetric energy highlighting that, depending on the propulsive powertrain operation, the better performing Li-Ion could still be of interest.

\subsection{ELECTRICAL ENERGY DISTRIBUTION AND CONVERSION}

A Hybrid Electric Aircraft has higher electric power requirements when compared to today's aircraft as the electric power is needed to (partially) supply the propulsive module and not only to supply the electric subsystems. For example, state of the art more-electric-aircraft such as the B787 employ around 1.5 MW aeroelectric system for non-propulsive "housekeeping" purposes [29]. On the other hand, regional aircraft have installed propulsive powers between 5 and $10 \mathrm{MW}[4,28]$ and although not all this power will be supplied by the electrical system, the electrical components will still require advances in technology before being safe and light enough to be employed with such large power requirements. This higher requirement is not easily satisfied with today's state of the art components as the power density and efficiency is not always enough. To circumvent this issue superconducting technology can be employed. This technology is presented in section 2.2.1.

The transfer of the power between the energy storages or generators and the electric motors should be as efficient as possible with minimal power loss in the form of heat. Enabling technologies and designing parameters for the high-power transmission are discussed in section 2.2.2.

The electrical energy can be produced and distributed in two ways: as direct current, DC, or as alternating current, AC. The power modules enable the conversion between different voltages and between AC and DC. 
Moreover, they can also be used to limit losses and control the engines. A brief description of power modules current technologies and their future developments is given in section 2.2.3.

\subsubsection{CRYOGENIC VS NON-CRYOGENIC POWER DISTRIBUTION}

\section{NON-CRYOGENIC}

Although the literature suggest that cryo-cooling and superconducting materials are the way forward for single and twin-aisle airplanes, a conventional non-cryogenic distribution system could be still feasible for regional aircraft $[4,30]$. The main benefit of conventional systems is that fewer components are needed as there is no need for the cryo-cooling system discussed below. This means that the electric system would be cheaper, simpler and more reliable at the cost of component's efficiency and weight.

The weight drawback could be limited or neutralized by carefully placing the electric components close together to minimise material used in the transmission or by limiting the power supplied by the electrical side of the hybrid system. However, these options would have an impact on the choice of HEP architecture as it will be discussed in chapter 3 and they could potentially restrict the maximum degree of hybridization (electric installed power over total installed power).

\section{CRYOGENIC}

Superconductivity is a phenomenon that enables electricity to travel inside certain materials, called superconductors, with minimal to no losses. A material becomes a superconductor when it is cooled below a characteristic critical temperature. This temperature can be extremely low $(<20 \mathrm{~K})$ and difficult to reach outside of lab setting. For this reason, researchers are focusing on the development of High Temperature Superconductors (HTS). HTS materials are super-conductive at temperatures above that of liquid nitrogen and are operated at temperatures between that of liquid neon $(27 \mathrm{~K})$ and liquid nitrogen $(77 \mathrm{~K})$. In figure 2.1 it is showed a time-line with the discovery of the most used super-conductive materials.

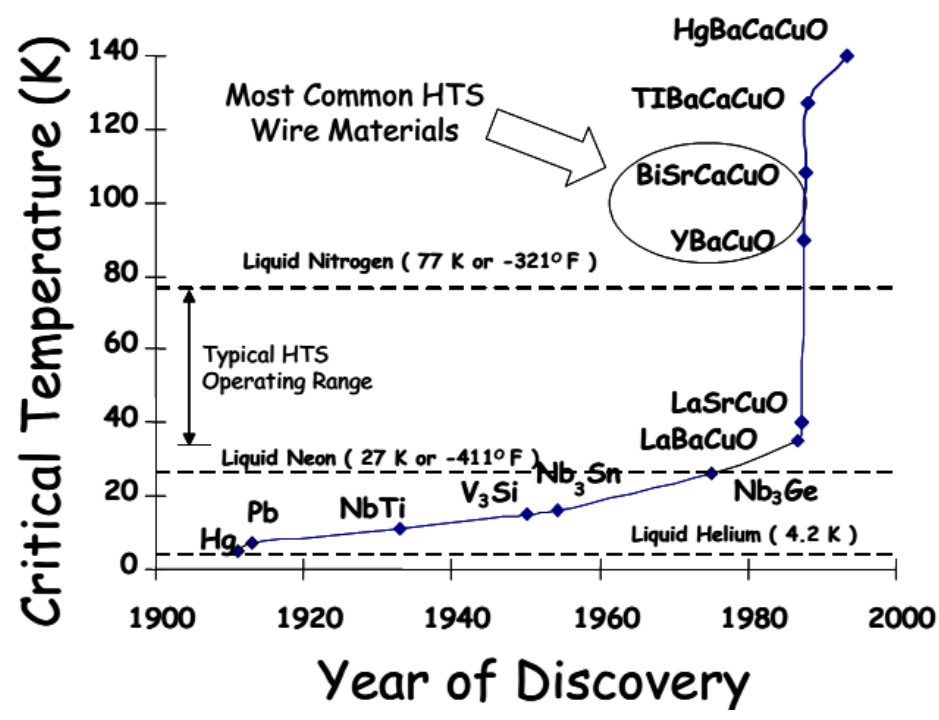

Figure 2.1: Critical temperature of superconducting materials [31]

Several authors [31-33] agree that HTS materials is one of the enabling technologies for hybrid electric and full electric aircraft propulsion when HEP architectures with very high electric power requirements (e.g. turboelectric) are chosen. This is due to the advantages that this technology brings to a high-power electrical system such as high-power density and low losses (high efficiency). The specific ways this technology influences the components will be described in the following sections.

Although the technology promises important advantages, it has also some drawbacks. The common one is that a new subsystem is needed on board of the aircraft to cool the components. Reference [34] and [30] introduce three different way a cryo-cooling system could work in an aircraft setting: 
1. Cryogen storage: the first way is to load cryogen at the airport that is enough for the entire flight plus a margin like the one used for fuel reserve. The insulated cryo-tank would then need to be refilled after each landing [30]. The main benefit is that it would minimize the weight of the system components by maintaining a low complexity. The main drawbacks are that the cryogen must be replaced after every flight and, if an open cycle is used, the choice of coolant is limited by environmental impact and cost reasons. It is also expected that the mass of cryogen will not be negligible when compared to the MTOW.

2. $H_{2, \text { liquid }}$ as cryogen: Like the previous point, it is suggested that to minimize the number of components the cryogen could be liquid hydrogen. Choosing this method would also increase the synergy of the cooling system as the cryogen can be used after it has been heated up since hydrogen can be burnt in a $5 \%$ to $95 \%$ ratio with fuel [30] or utilized in fuel cells to generate electricity. This method shares the same disadvantages as the previous one (need for on-ground refuelling facilities, large insulated tanks) and it also introduces the need for a complex fuel/coolant control system. Moreover, its biggest disadvantage is the poor energy density of hydrogen that requires heavy high pressure and insulated tanks to carry enough cryogen for the flight.

3. Cryocoolers: The third option is to use an active source of cooling that keeps the cryogen in the closedloop system at the required temperature. This method is the preferred choice as it is a "plug and play reliable system" [30] that can undergo the same maintenance cycles as the other components and it does not require neither much additional volume or ad-hoc facilities on ground for refuelling.

Another important drawback for superconducting technologies is that, at the moment, there are no suitable materials for alternating current (AC). This is an issue that severely limits all superconducting components as both motors and generators are at least partly working with AC. Regarding the transmission, it could be problematic for DC (Direct Current) cables as well if the system experience large transients [4]. To avoid transients, the distribution system must be de-coupled from the generators and motors with active controllers [35]. This adds weight and reduces the overall efficiency of the system.

\section{CRYO-COOLER}

A cryocooler is a refrigerator that can produce temperatures below $100 \mathrm{~K}$. It is typically connected in a closedcycle that pumps the refrigerant to the parts that need to be brought to cryogenic temperatures. The main figures of merit of a cryocooler are its weight to power ratio and its fraction of Carnot Efficiency. They are a major roadblock to the superconducting turbo-electric and hybrid-electric aircraft due to cost, weight and reliability $[8,34]$.

Regarding the weight, the most massive parts are usually the compressor with its drive motor and electronics [36] and the cold-head [30]. Proposed changes to make cryocoolers viable for airborne applications are to use an efficient turbo-compressor and use aeronautical materials such as aluminium and titanium for the cold-head [30].

State of the art cryocoolers achieve a power to mass ratio of around $0.06 \frac{\mathrm{kW}}{\mathrm{kg}}$ and $15 \%$ of the Carnot efficiency $[30,36]$. This is still too heavy for aerospace applications. However, an advanced study by NASA has produced a design of a turbo-Brayton cryocooler that achieves $30 \%$ of the Carnot efficiency with a reduced power to mass ratio of $0.3 \frac{\mathrm{kW}}{\mathrm{kg}}$ [34]. This encouraging design needs further validation but it promises to satisfy the requirements of airborne electric propulsion.

Regarding reliability, it is suggested to avoid using only one cryo-cooler as it would be a single point of failure and it would require long tubing to dispatch cooling everywhere it is needed. The suggested redundancy is one or two cryocoolers per generator and one every two electric motors [28]. The risk of so many added components is somewhat balanced out as the low temperatures reduces the degradation of insulation caused by thermally activated processes[34]. As the rate of these processes goes down exponentially with the temperature, this major cause of failure in high power electric systems is drastically reduced.

Another issue presented in the literature [4] is that if the cryo-cooler is sized only on the maximum thermal load, it could take days to cool the entire propulsive system from ambient to operating temperatures. Speeding up the process requires both an oversized cooling system while the cooling distribution system needs to withstand the resulting thermal shock. 


\subsubsection{POWER DISTRIBUTION}

\section{NON-CRYOGENIC}

With the advent of more-electric-aircraft designs the aero-electric system has grown to be able to carry around 1.5 MW of power. This high-power transmission is reached on the Boeing 787 thanks to the higher system voltage of $\pm 270 \mathrm{~V}$ (or $540 \mathrm{~V}$ ) [4]. However, this voltage is the maximum achievable with current cable's technologies as it is close to the physic limit referred as Paschen curve limit ${ }^{1}$. Since regional transports could require between 5 and $10 \mathrm{MW}$ of electric power just for propulsive needs, it is necessary to use cables that can safely carry voltages in the order of kilo-volts to maintain current and its related losses to reasonable values. This requires new insulation, electrical conductor's materials and spacing rules [4].

Cables are composed by a conductive core and three layers around it:

1. Core: it is made of a conductive material with enough current density to transfer the power while maintaining reasonable weight and cost. Common materials are copper and aluminium and new materials that are being studied are based on novel technologies such as polymers and carbon nanotubes [4, 35].

2. Dielectric layer: this layer resists the potential between the core and the magnetic shield layer. Its thickness is thus proportional to the nominal voltage. It is usually the most delicate part of the cable since the materials used degrades through thermochemical induced processes.

3. Magnetic shield: this layer is needed to shield the conductive core from current induced magnetic fields. It is usually made of aluminium and its thickness is proportional to the current.

4. Cooling sleeve: high power cables might need an active source of cooling to keep the temperature below the maximum allowable. Excessive temperature can damage the cable (mostly the dielectric layer) and the surrounding electronics and delicate components. The maximum temperature is usually set at $125^{\circ} \mathrm{C}$ [35] with operating temperature around $50^{\circ} \mathrm{C}$. The coolant can be water or oil-based.

\section{CRYOGENIC}

Superconducting cables promise very high-power densities and near lossless power transmission [28]. This is true for DC transmission but AC power transmission with state-of-the-art materials is yet not satisfactory $[31,34]$.

The structure of superconducting cables is also very different as it is commonly made of a tape consisting of several layers. The structure of the layer is shown in figure 2.2a. To efficiently cool the superconducting material, the tape is wrapped around a "former" [35] to give it shape and structural resistance. The wrapped former is then inserted in concentric tubing in which the cryogen is pumped and finally a thermal protection is put around to insulate the cable. The complete structure is shown in figure $2.2 \mathrm{~b}$.

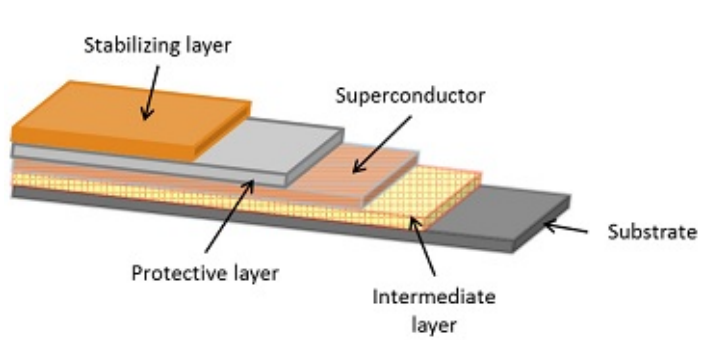

(a) HTS tape structure.

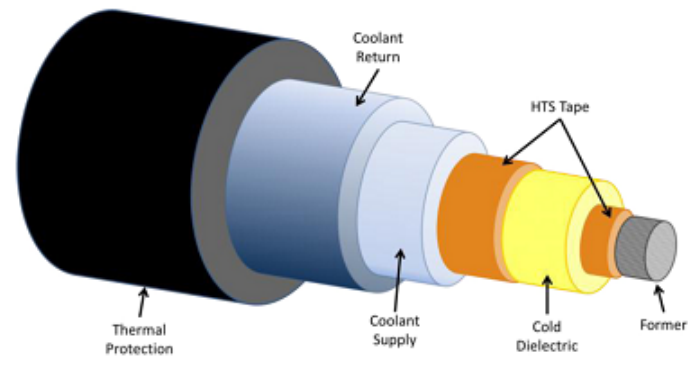

(b) HTS cable structure

Figure 2.2: Superconducting tape and complete cable structure [35]

State of the art super-conductive materials are YBCO and BSCCO that are cooled by liquid hydrogen and liquid nitrogen respectively.

\footnotetext{
${ }^{1}$ Paschen's law gives the voltage necessary to start a discharge between two electrodes given certain environment variables such as medium, pressure and gap length.
} 
The superconductive cable chosen in the design of the NASA N3X turbo-electric distributed propulsion design [29] weights $9.2 \mathrm{~kg} / \mathrm{m}$ and can carry $70 \mathrm{MW}$ with losses of only $5 \mathrm{~W} / \mathrm{m}$ if it is maintained below $77 \mathrm{~K}$ (liquid Nitrogen) [37]. Although this cable is clearly oversized for anything concerning a regional aircraft, it clearly shows what it is meant when the technology is presented as the solution for lossless high-power transmission. Naturally, if cryogenic power distribution is chosen for a specific design, it is essential to account also for the cooling power and cryo-cooler mass.

\section{AC VS DC ARCHITECTURES}

The transmission and distribution between the power sources and power users can take place in the form of direct or alternating current. The decision between the two is dependent on the type of electric propulsive system architecture. All in all, it appears that DC transmission offers more advantages for a HEP[35]:

1. If the system is using HTS materials and operates at cryogenic temperatures the cable has virtually no losses.

2. Batteries and other energy storages (fuel-cells, ultra-capacitors) can be connected only to DC systems.

3. If the motors can produce regenerative power, it can be easily returned to the bus for charging the energy storages or powering other motors.

4. Generators and motors are electrically de-coupled and they can run at the speed for their optimal performance.

On the other hand, DC systems needs rectifiers and inverters (section 2.2.3) if AC machines such as generators are connected to the DC bus. Moreover, they need controllers to keep the line free from transient loads. All these components increase the bulk of the system and decrease its efficiency.

Purely AC systems are limited to turbo-electric architectures as no batteries can be connected on the power bus. If the AC lines are efficient enough, this choice is the lightest as the generators are directly connected to the motors without the need for converters. Two drawbacks make this option less interesting: firstly, the controllability of the electric machines is sacrificed as the generators and motors' rotational speeds are coupled at a fixed ratio, like in a mechanical gearbox. Secondly, if one motor stops, it could prove impossible to start again while the system is under load [29]. For these reasons, in this study only DC power distribution will be modelled.

\subsubsection{POWER MODULES}

Electric systems need a way to transform and control the power flowing through the cable. The power modules used to satisfy this task are[28]:

1. Rectifier: it converts current from AC to DC. A rectifier would be needed if an electric generator is attached to a DC bus.

2. Inverter: it converts current from DC to AC. Inverters are needed to power the motors if the current is supplied as DC.

3. Transformer: it is used to change the voltage between DC-DC or AC-AC. DC transformer can be used to better integrate energy storages such as batteries and fuel-cells. AC transformers can be used to change the speed ratio between generators and motors in a turbo-electric configuration.

4. Circuit protectors: electronic circuit protection needed for the safety of the electrical system.

5. Mechanical breakers: mechanical circuit protections can be used with higher power systems but they are heavier and more complex than the electronic version.

The first three fall under the category of power converters while the last two falls under the category of circuit protectors. Technology expectations for conventional and cryogenic power modules are presented in the following sections. 


\section{NON-CRYOGENIC}

State of the art power converters are relatively efficient at 95\% [28], although this is still low for a high-power electric system as the wasted heat could be a large fraction of the expected fuel saving benefit. This becomes even more apparent if it is considered that power converters are connected to generators, motors and the distribution system in a HEP and thus the efficiency drops to values between $70 \%$ and $80 \%$ when considering the overall system [4]. Moreover, state of the art power electronics has capabilities up to only $0.25 \mathrm{MW}$. A possible solution with today's technologies is to use more power converters in parallel. This increases the efficiency and power capability in exchange for a higher weight [28].

Both power converters and electronic circuit protectors have similar specific power that today are around $2.2 \mathrm{~kW} / \mathrm{kg}$. It is envisioned that by 2035 the specific power will increase up to $9-15 \mathrm{~kW} / \mathrm{kg}$ and the electronic protectors will be usable up to $108 \mathrm{~kW}$ instead of being limited at $7 \mathrm{~kW}$ as today [4]. It is also expected that the power capability will improve by an order of magnitude to 1-3 MW [4] making their use viable for regional and smaller single-aisle HEA.

\section{Cryogenic}

Some cryogenic power converters have already been tested and, although the efficiency remained around $95 \%$, the specific power already surpass $8 \mathrm{~kW} / \mathrm{kg}$. Improvements in the use of superconducting technologies will allow to further push this boundary by decreasing internal resistances, increasing switching speeds and improving heat transfer [34]. It is expected that future cryogenic power converters will process $16.5 \mathrm{~kW} / \mathrm{kg}$ at $98.8 \%$ efficiency $[28,34]$.

Even more ambitious goals put the expected performance at $25 \mathrm{~kW} / \mathrm{kg}$ and $99.5 \%$ efficiency [38]. In both cases the cryo-cooler system is considered in the models. No information was found on cryogenic circuit protectors but it is expected that the electronic version will have similar characteristics to the power converters.

\subsection{Motor And Generator technology}

The last components that need to be discussed to have a complete picture of the electric part of the HEP system are the active power producers and users: generators and electric motors. At a high level of abstraction motor and generator can be modelled in the same way with the only difference being the electric power direction. There are other differences between the two that are due to their intended use since motors and generators differ mostly in their rated power and rotational speeds [34].

Generators for HEP systems can be designed such as their shaft speed is equal to the optimum speed of the power turbine (6500 and 15000 RPM designs where found in the literature [32]). This design option brings benefits both to the thermal engine and generator since a gearbox can be omitted, saving weight and efficiency loss, the power turbine can rotate at higher speeds than if it was directly connected to a fan, allowing it to be made smaller and lighter and finally the generator has a higher power density [34]. The upper limit to the rotational speed of the generator's rotor is given by mechanical and electrical reasons. The mechanical limit is caused by the high centrifugal forces that the rotor windings must sustain, the electrical one is due to the high heat that is generated in high power machines and the resulting high voltage as its proportional to the rotational speed.

The motors on the other hand can be either designed with shaft speeds compatible with fan or propeller optimal speeds, both slower than optimal turbo-machinery speeds or to be directly connected on the freeturbine of a turbo-shaft engine in case a parallel HEP architecture is chosen. For a fixed machine design (windings number and type, machine type and control system, materials used etc.) the rotational speed is proportional to the applied voltage and the torque is proportional to the current. Thus, the slower speed means that, for the same machine type and power, the motors will have lower applied voltages and higher current when compared to high-speed generators. The higher current will cause higher power losses, known as Ohmic losses, due bigger heat production as described by Joule's first law ${ }^{2}$. The higher losses must then be offset with a more powerful machine and heavier cooling system. On the other hand, connecting the motor directly to the propeller offers savings in terms of weight, efficiency and parts number as no gearbox is

\footnotetext{
${ }^{2}$ Joule's first law states that an electric current through a conductor produces heat power proportional to the conductor resistance and
} to the square of the current. 
required. Distributed propulsion offers a good solution to this issue since the propulsive power can be spread over more motors keeping them light and efficient.

In general, electric machines are reliable and have very long life-cycles. Their specific power should be increased further to make them more appealing for aeronautic purposes. Motor's performance requirements for parallel hybrid will be between 1-3 MW total propulsive power and at least $3 \mathrm{~kW} / \mathrm{kg}$ while for series architectures motor requirements are between 1.5-4 MW and 1-11 MW for the generators with at least $6.5 \mathrm{~kW} / \mathrm{kg}$ specific power [4]. State of the art performance and future expectations are discussed in the following sections differentiating the expectations between conventional machine and more technologically advanced cryogenic ones.

\subsubsection{NON-CRYOGENIC POWER MACHINES}

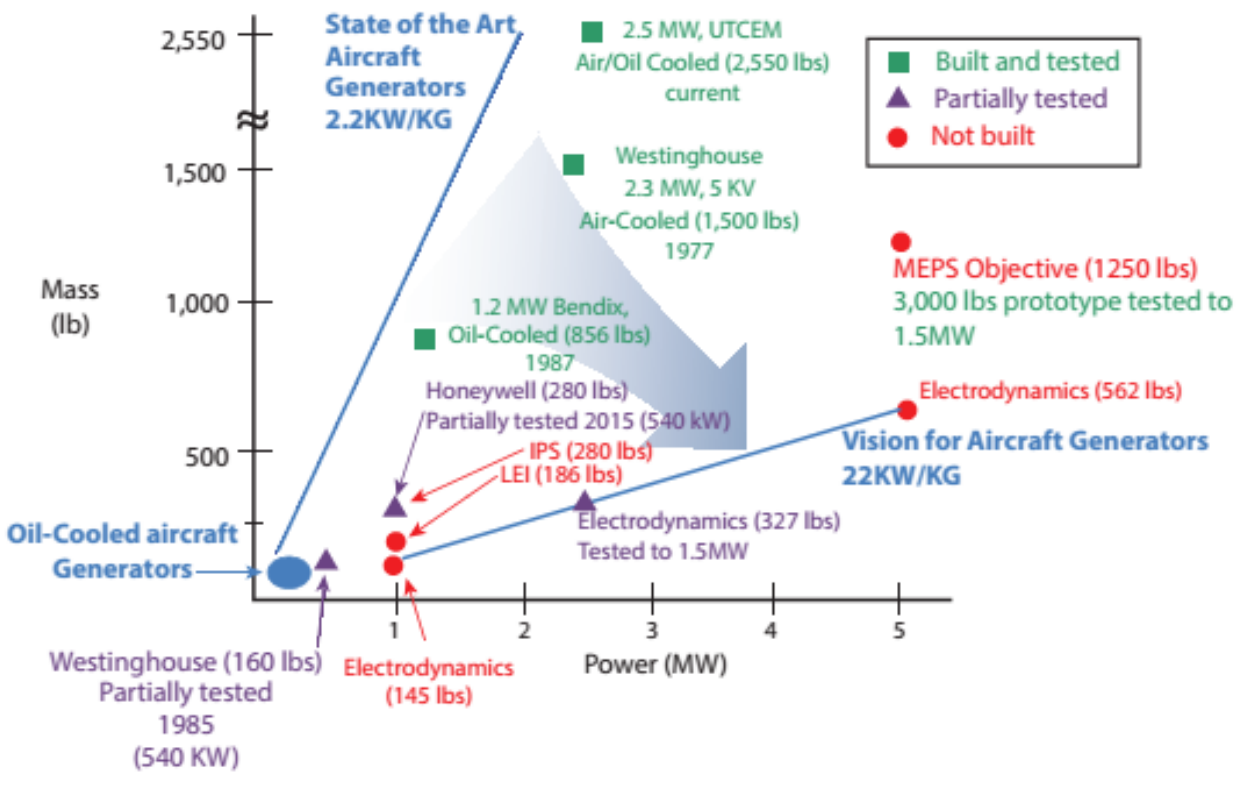

Figure 2.3: Aircraft megawatt generator research, development and vision [4]

State of the art electric machines are mostly being used in cars, ships and industrial applications. Simply scaling these designs is not a viable solution since their specific power are greatly reduced by aeronautical requirements such as stringent operating environment and unique safety certifications that add to their size and weight. State of the art non-cryogenic machines achieve specific powers of $2.2 \mathrm{~kW} / \mathrm{kg}$ and are limited to 0.25-0.5 MW of power. However, when considering the associated electronics, the weights double lowering the specific power to roughly $1.1 \mathrm{~kW} / \mathrm{kg}$ [4]. As stated above, this performance level is not deemed enough for hybrid electric propulsion $[4,30]$.

A solution to reduce size and weight of these machines is to increase their design shaft speed, increase generation and distribution voltage and increase conversion efficiency. The obstacles to these improvements are the limits imposed by mechanical stresses, the breakdown (or electric arc) voltage given by Paschen's law and the material limitations of silicon-based power electronics.

Future expectations vary between $9 \mathrm{~kW} / \mathrm{kg}$ for motors and a very ambitious goal of $22 \mathrm{~kW} / \mathrm{kg}$ for generators with power levels between 1-3 MW [4] shown in figure 2.3. Good results are already being achieved as demonstrated by Siemens new aeronautical grade electric motor. This motor produces up to $261 \mathrm{~kW}$ of mechanical power with a specific power of $5 \mathrm{~kW} / \mathrm{kg}$ and 95\% efficiency [39]. An Extra300 powered by this motor has recently set world records for climb performances, straight flight speeds and for being the first electric tow plane. Siemens declared that it is looking to scale this design up to $1 \mathrm{MW}$ to start testing solutions for future transports aircraft in collaboration with Airbus [39, 40]. 


\subsubsection{CRYOGENIC POWER MACHINES}

State of the art cryogenic machines are usually not fully superconducting as only the DC parts are. The components that work in AC, although they can be brought to cryogenic temperature, are not superconductive since a proper material has yet to be found [34]. In addition, cooling the rotor presents mechanical challenges due to the high rotational speed and complex design of superconducting wires [30]. Overall, the literature reports specific powers between 3 and $8 \mathrm{~kW} / \mathrm{kg}$ (considering cryo-cooler mass) making semi-superconducting machines slightly lighter than conventional ones and on comparable level with turbine engines estimated at around $5 \mathrm{~kW} / \mathrm{kg}[30,32,33]$. The benefit of these machines is thus expected to derive mostly from their higher efficiency.

The areas where cryo-machines offer greater advantages are their rated power and efficiency. Semi superconducting machines already can be scaled up to $1.5 \mathrm{MW}$ and they have improved overall efficiency of 98.5\% [34] making them already feasible options for the motors of small regional aircraft. Moreover, the very low internal resistance of the windings and the high voltage that they can carry allow very high rotational speeds to be achieved making the machines $25 \%$ smaller in volume when compared to conventional designs [34].

A notable machine is the generator designed by General Electrics [32]. This machine has a specific power of $7 \mathrm{~kW} / \mathrm{kg}$, power rating of 1.3 MW and 97\% efficiency (considering cryo-cooler power draw) at 10'000 RPM. This rotational speed has been made possible by putting a magnetic core in the rotor instead of having wire windings thus making the rotor more resistant to the high centrifugal stresses. Moreover, only the stator needs to be cooled and the coils here can be optimized without worrying about the rotational stresses allowing the use of more efficient insulators and cooling interfaces.

Regarding future expectations, the roadblock is finding an AC superconductive material. If that is achieved, fully superconducting machines can be designed. Specific power expectations for these machines are around $25 \mathrm{~kW} / \mathrm{kg}$ for motors and $50 \mathrm{~kW} / \mathrm{kg}$ for generators making them between two and three times lighter than same power non-cryogenic machines [30]. 


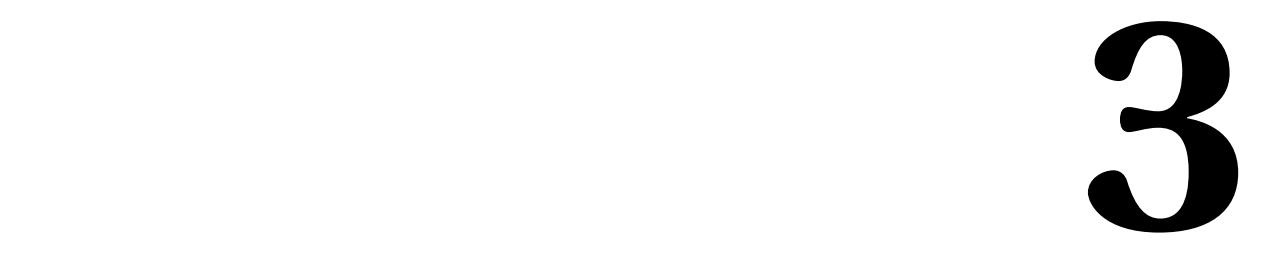

\section{Qualitative ASSESSMENT OF HEP CONFIGURATIONS}

In a hybrid power train different energy sources and their relative power conversion devices are combined to achieve a better overall performance than the one that would be obtainable by the sub-systems taken alone [41]. In this study, the energy reservoirs are electro-chemical batteries and jet fuel. The power converters are electric generators, electric motors and gas-turbine engines. The other components that can be used in the HEP configuration are fuel lines, mechanical transmissions and the Power Management And Distribution system (PMAD) that groups together all the electrical components required for the transmission and safe use of electric power (cables, inverters, rectifiers, transformers and circuit breakers).

Due to the multi energy sources characterizing hybrid systems, the design space for the possible propulsive system architectures is larger than in the conventional case. In the following sections a useful way to describe the electrification of HEP system is described. Then, the most studied and common HEP architectures for aviation purposes are presented and qualitatively described. The result of this work then forms the base for the assumptions necessary to define the design parameters used in the proposed HEA sizing method.

\subsection{Degree of Hybridization}

To classify a hybrid electric powertrain, it is necessary to describe both its configuration and the subdivision of the propulsive power provided by the sub-systems. Lorenz et al. [42] defined the Degree of Hybridization as:

$$
\mathrm{DoH}_{P}=\frac{P_{\text {em }}}{P_{\text {Tot }}}
$$

This definition is not the only one that is found in the literature to describe the power split in a hybrid system but it is the most widely used when describing aircraft's HEP.

In equation 3.1, $P_{\text {em }}$ is the power output of the electric engine and the total power, $P_{T o t}$, is the sum of $P_{\text {em }}$ and the output power of the gas turbine, $P_{g t}$ [42]. This parameter easily describes how much the sub-systems contribute to the total output power but it does not give any information about the split in energy storage. For this reason, a second parameter, the energy-based degree of hybridization, is introduced:

$$
D o H_{E}=\frac{E_{b a t}}{E_{T o t}}
$$

In this case, $E_{\text {bat }}$ stand for the energy stored in the batteries and the total energy, $E_{\text {Tot }}$, is given by the sum of $E_{\text {bat }}$ and $E_{\text {fuel }}$ where the latter is the energy stored as fuel. Both the parameters are bound between 0 , fully conventional power/energy source, to 1 , fully electrical propulsive power/energy source. 


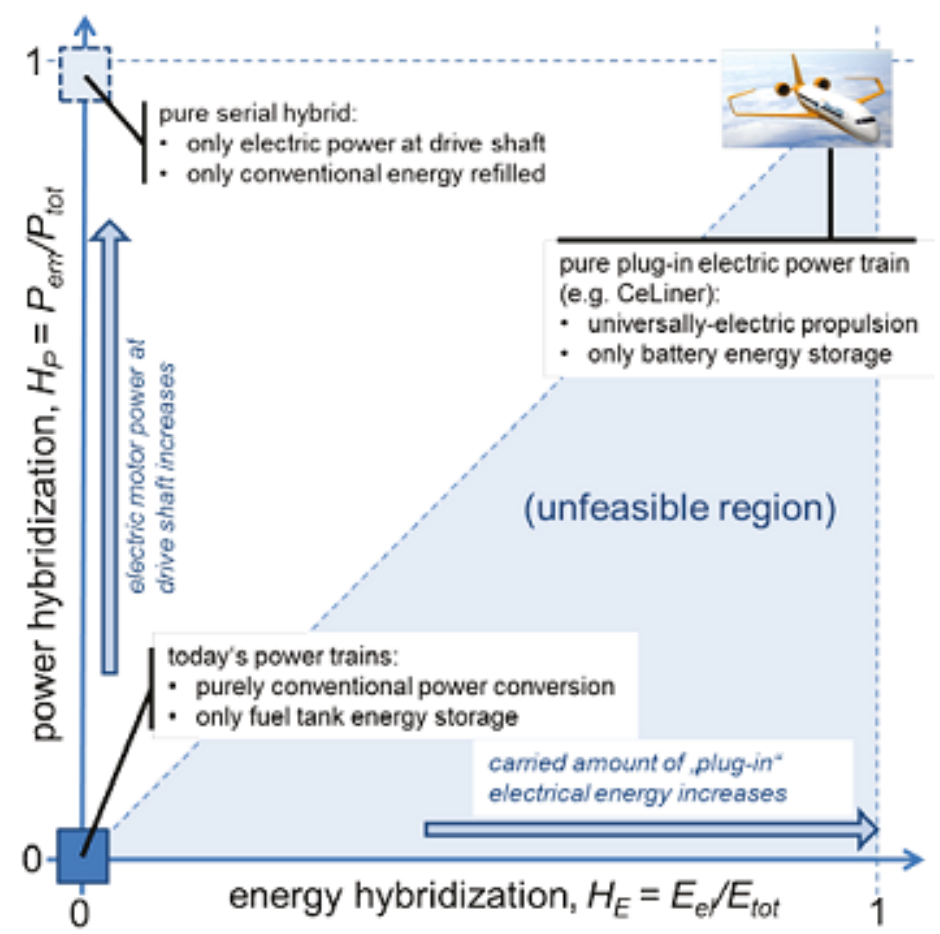

Figure 3.1: Degree of Energy and Power hybridization to describe HEP [42]

The graph shown in figure 3.1 illustrates the space for classifying hybrid electric powertrains and it contains some interesting characteristics. First, the line diagonally crossing the graph separates the space in a feasible and an un-feasible area. The un-feasible region exists since it does not make sense to store more electric energy than the one that the motors are able to extract (outside of the required reserves for critical conditions).

The line will not be linear as the slope varies depending on HEP architecture and the airplane mission. For example, altitude effects influence the slope of the boundary in a not negligible way [42]. Moreover, the slope could be more or less inclined depending on the relative efficiency of the sub-systems. For example, if the electric system has a higher efficiency than the conventional one, the slope will be higher as less electrical energy is needed for the same amount of electrical power output.

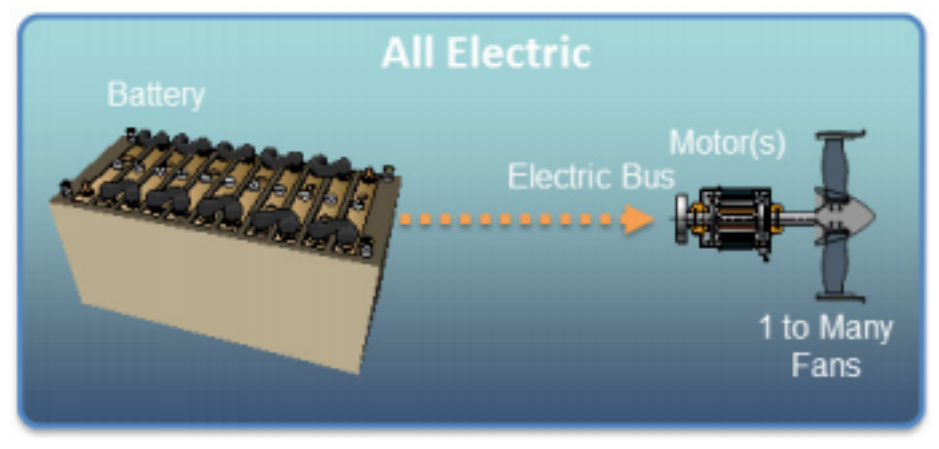

Figure 3.2: Universally electric architecture [4]

Other interesting parts of the graph are the edges of the graph at $(0,0),(0,1)$ and $(1,1)$. The first point identifies today's conventional propulsive system architecture where all energy stored for propulsion is in the form of fuel and the propulsive power is entirely produced by conventional gas turbine engines. The second point, $(0,1)$, identifies a pure serial configuration. This architecture is also called turbo-electric and, while the energy is stored only as fuel, the turbine is connected to a generator-motor system that produces $100 \%$ of the needed 
propulsive power. The last point, $(1,1)$, identifies the universally-electric concept where no fuel is used and the complete system uses only electricity [42] as showed in figure 3.2.

A commercially interesting universally electric aircraft requires high gravimetric energy density in the order of $1800-2000 \mathrm{Wh} / \mathrm{kg}$ and values of specific power for the electric motors that can be achieved only with the use of fully-superconducting machines [43]. As illustrated in the previous chapter, these performances will not be achieved by the year 2035, thus this architecture will not be further expanded upon in this document.

\subsection{SERIES AND TURBO-ELECTRIC CONFIGURATION}

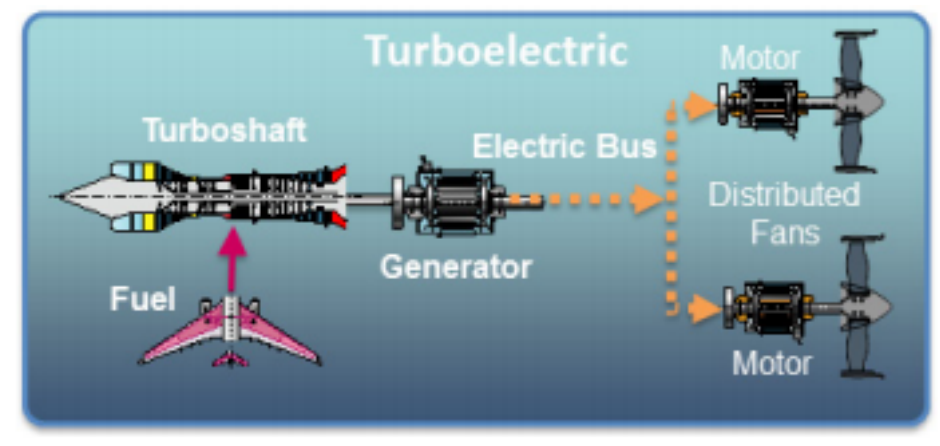

Figure 3.3: Pure series or Turbo-electric architecture [4]

The series configuration is defined by a $\mathrm{DoH}_{P}=1$ as all the propulsive power is produced by electric motors. Depending on the energy degree of hybridization there is then a distinction between pure serial (turboelectric), $D o H_{E}=0$ [44] and series hybrid configuration, $0<D o H_{E}<1$. All the series configurations share some common components such as fuel tanks, gas turbine engines, generators, electric power lines and electric components grouped in the PMAD, electric motors and finally the propulsive units (propeller or fan). Besides the turbo-electric configuration, the others have also an electric energy reservoir, usually in the form of batteries.

The pure series architecture, showed in figure 3.3, is distinct from others as it does not depend on advances in energy storage technologies. However, as discussed in the previous chapter, the high-power requirements require further advances in power electronics and power machines technology. The absence of electrical storage means also that the gas turbine engine must be sized for peak power demand thus nullifying some of the expected advantages of hybrid electric systems. The turbo-electric power train is also described as being less efficient and heavier when compared with the direct mechanical connection of the fan-turbine system or the propeller-gearbox-turbine system [41]. For this reason, if this configuration is applied directly to a conventional design it would probably fail to be competitive and it would not meet the expectations of reduced fuel burn and environmental impact. The saving point for turbo-electric power trains is that they offer a great deal of flexibility in the number and position of the electric motors thus it is a system that readily adapts to be used in non-conventional concepts such as distributed and boundary layer ingestion (BLI) designs [44].

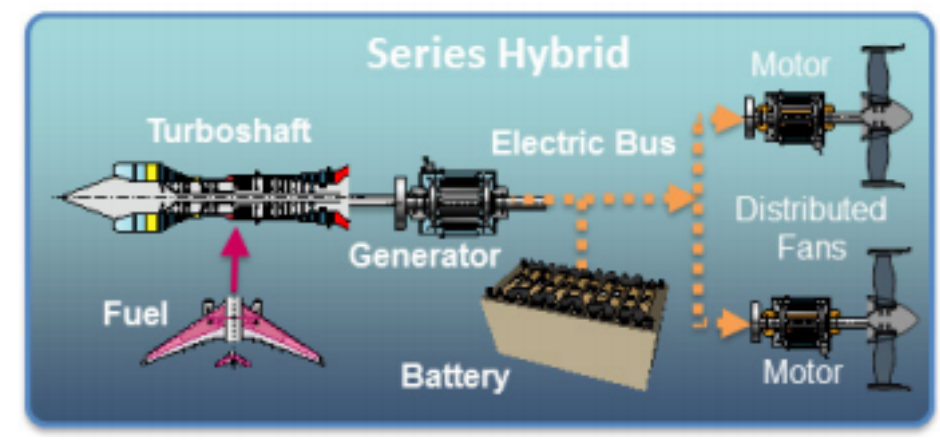

Figure 3.4: Series hybrid architecture [4] 
Series hybrid configurations, showed in figure 3.4, differ from the turbo-electric ones by having an electrical energy storage connected to an electric node before the electric motor. This configuration shares some disadvantages as the purely series one, namely higher weight and losses due to the many energy conversions $[45,46]$, but it partially balances them by having a more efficient gas turbine engine. This is because the engine is decoupled from the propulsive part and it can run at optimal speed. Moreover, depending on the energy degree of hybridization, $\mathrm{DoH}_{E}$, the engine can be smaller since it needs to supply only average power demands while the batteries are used to supply the rest during peak demand [45]. On the other hand, making the thermal engine too small and light could bring to an overall weight penalty due to the worse performance of batteries and electric power modules.

Another advantage of the series system, shared also by turbo-electric configurations, is that it is the most simplistic as there is no need for complex mechanical connections between the propeller, gas-turbine and electric motors [47]. Moreover, as for the turbo-electric configuration, there is a lot of flexibility in aircraft-engine integration thus allowing the use of many smaller fans/propellers distributed in effective ways [46, 47]. Lastly, the system's PMAD can be designed to allow two-ways current flow thus enabling the in-flight re-charge of the batteries. For example, this can happen during the cruise phase if the gas-turbine is run at a higher power than required from the propulsive device or, if a regenerative propellers technology is used, during the descent phase by converting part of the kinetic and potential energy into electrical energy [48].

\subsection{Parallel configuration}

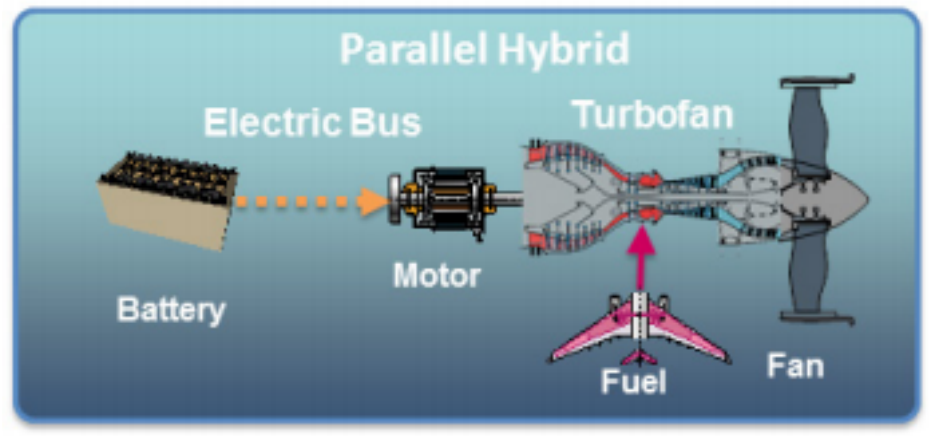

Figure 3.5: Parallel architecture [4]

Parallel configurations, shown in figure 3.5, are defined by having a mechanical node in which the power from conventional and electric engines are added together to drive a propulsive device [42]. This type of architecture has degree of hybridization $\mathrm{DoH}_{P}$ between 0 and 1 as at least part of the propulsive power is supplied by the conventional gas turbine engine without any conversion into electrical power. Common components of parallel configurations are fuel tanks and engines for the conventional sub-system and batteries, controllers and electric motors for the electrical one. Differently from the series architecture, there are no electric generators supplying power for propulsion.

The mechanical node can be direct (electric motor shaft is connected to the gas-turbine power shaft) or a gearbox with two input shafts. Depending on the configuration, the gearbox inputs can be always coupled or the shafts can be de-coupled with the use of clutches. The latter is feasible for small airplanes but having clutches in high power and high-speed power drives is not efficient and easily done [49]. Moreover, although this system allows the option of driving the propeller with only one power source, it does not allow a continuous control as the clutches should not slip and when they are locked, the speed ratio between motor and thermal engine is fixed.

On the other hand, if the shafts are always coupled directly or via a gearbox, it will result in less controllability as stopping the gas turbine engine is not an option and freewheeling the electric motor could cause a significant drag on the thermal engine [45]. The switched reluctance electric motors offer very little drag when not powered and they are thus one of the preferred solutions for parallel configurations [50].

Although the instantaneous power split between electrical and conventional system can be changed in flight, its range is more limited when compared to the series one as lowering too much the conventional power input 
would severely impact the turbine engine efficiency and could shut the engine off [47]. Another drawback of the dependency between conventional and electric systems is that the engine cannot be used to charge the batteries via a generator as in the series configuration. In flight charging is viable only if a motor/generator is used and the mechanical node splits the power between charging and supplying propulsive power citeArch4. Motor/generator are more complex and heavier than a normal electric motor due to the added electronic components required [4].

Parallel hybrid configurations have the additional benefit of redundancy since there are at least two power inputs that can drive the propulsive units [50] as ong as the machines ratings are comparable. The power degree of hybridization can be set also at low values and this translates positively if the specific power and efficiency of the conventional sub-system is higher since the HEP can be optimized for minimum weight. This is the main reason for which parallel systems are reported as being lighter than the series counterpart [46] where the totality of the propulsive power must be supplied electrically. A final drawback of this configuration is that it limits the aircraft design in the choice of aircraft/propulsion integration since long mechanical connections are not feasible for these high-power systems.

\subsection{SERIES-PARALLEL CONFIGURATION}

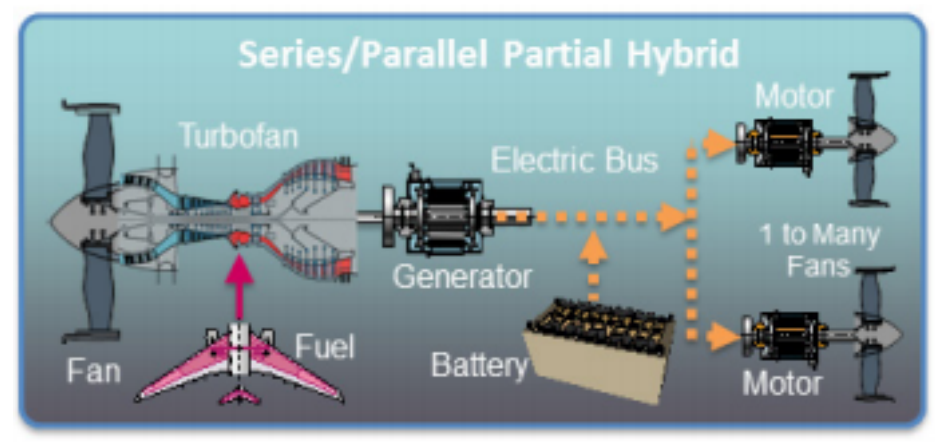

Figure 3.6: Series-Parallel hybrid architecture [4]

The series-parallel configuration shown in figure 3.6 is an architecture that aims to combine the advantages of the architectures discussed so far. It is characterized by power degree of hybridization between 0 and 1 and it possess both a mechanical and an electrical power node.

Different authors use this name for different configurations. For example, the authors of reference [4] use this name to describe a system in which the gas turbine engine is connected to a mechanical node (fan-shaft or propeller-gearbox) and to a generator. The power output from the generator is then directed to an electric bus that provides power to motors that are not connected in any mechanical way with the thermal engine. On the electric bus it is also present an energy storage that can supply/draw power from it [4]. Other authors such as the one in reference $[47,48]$ have the electric motor connected to the mechanical node of the turbine engine thus closely resembling both the series and parallel architectures. The advantage over parallel configuration in this case is that the batteries can be re-charged in flight.

As previously described, the parallel-series configuration tries to combine the advantages of both systems but in doing so it is plagued by a heavier weight and a more complex control strategy [47]. This is because there is an additional mechanical link when compared to series hybrid and additional generator and control electronics when compared to a parallel system [48].

\subsection{PARTial turbo EleCtric CONFIGURATiON}

The last configuration described is very similar to the parallel-series architecture with the only difference being that no battery is used in this configuration as shown in figure 3.7. This means that the gas-turbine drives both a mechanical shaft connected to a propulsive unit and to a generator, yet it does never receive power from it. 


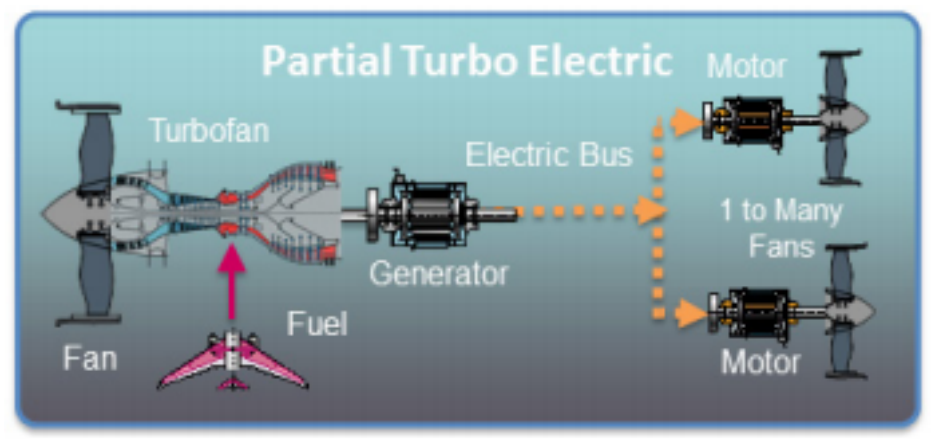

Figure 3.7: Partial series hybrid architecture [4]

This configuration is not widely discussed in the literature [4] but it should offer similar advantages as the series configuration while alleviating the power demands from the electrical propulsive sub-system. In this way, part of the flexibility in positioning the turbine engine is sacrificed for a less powerful, thus lighter, electric propulsion system.

This configuration would maintain conventional positions of the thermal based propulsion system and it would obtain further benefits by smartly positioning the electric motors. It would make sense to try to find places where the propulsion power would synergies with other factors. For example, by positioning small electric motors at the wing tips, the swirls produced by the propellers could help in counteracting the lift generated vortexes thus alleviating lift induced drag. Propellers in this position could also be used as an active yaw control, possibly allowing for a downsize of the vertical tail. Finally, small propellers/fans could be used to fill the fuselage or wing wake as in the concepts of BLI and distributed propulsion designs. This system would probably suffer from disadvantages of both series and parallel architectures. Depending too much on the electrical system could have weight penalties while lacking the battery electric node decreases the redundancy of the system.

\subsection{QUALITATIVE COMPARISON}

In table 3.1 a summary of the advantages and disadvantages of the discussed configurations is given. In general, researchers in the literature opt for a parallel architecture as it proves to be the lightest and it can be easily used to retro-fit a conventional aircraft [51]. However, it is observed that if the series architecture is properly integrated with the aircraft, important benefits could arise, making it an interesting option in the design of HEA. This fact can also be observed in the proposed turbo-electric and series designs briefly described in the introduction: Airbus E-Thrust [10] and ESAero EC-150 [12] both use distributed fans on the wing to increase equivalent bypass ratio (increasing propulsive efficiency) and to fill the aircraft wake (decreasing drag). For this reason, it is worthwhile to consider more architectures in the design process and observe the impact at aircraft level and not only at the propulsive system one. 
Table 3.1: Summary of advantages and disadvantages of HEP configurations

\begin{tabular}{|c|c|c|}
\hline $\begin{array}{l}\text { HEP con- } \\
\text { figuration }\end{array}$ & Advantages & Disadvantages \\
\hline $\begin{array}{l}\text { Turbo- } \\
\text { electric }\end{array}$ & $\begin{array}{l}\text { Does not depend on advances of en- } \\
\text { ergy storage technologies } \\
\text { - High design freedom for propulsion- } \\
\text { aircraft integration }\end{array}$ & $\begin{array}{l}\text { - High weight and low efficiency } \\
\text { - Gas turbine engine is sized for peak } \\
\text { power conditions } \\
\text { - Depend on advances in HTS material } \\
\text { technology }\end{array}$ \\
\hline Series & $\begin{array}{l}\text { Decoupled engine can run at optimal } \\
\text { RPM throughout mission } \\
\text { - High design freedom for propulsion- } \\
\text { aircraft integration } \\
\text { - Power split between conventional } \\
\text { and electrical power source is ad- } \\
\text { justable in flight } \\
\text { - Batteries can be re-charged in-flight }\end{array}$ & $\begin{array}{l}\text { - High weight and low efficiency } \\
\text { - Need a generator } \\
\text { Could depend on advances in HTS } \\
\text { material technology }\end{array}$ \\
\hline Parallel & $\begin{array}{l}\text { - No need for a generator makes it } \\
\text { lighter } \\
\text { - Fewer energy conversions, more effi- } \\
\text { cient } \\
\text { - Power split between conventional } \\
\text { and electrical power source is ad- } \\
\text { justable in flight } \\
\text { - Engine could be down-sized to pro- } \\
\text { vide only average continuous power }\end{array}$ & $\begin{array}{l}\text { - Could need a complex gearbox } \\
\text { Power split is changes are restricted } \\
\text { due to risk of engine off-design oper- } \\
\text { ation } \\
\text { - Engine is not decoupled from thrust } \\
\text { and cannot run at optimal RPM } \\
\text { - Mostly limited to conventional con- } \\
\text { figurations for engine-aircraft inte- } \\
\text { gration }\end{array}$ \\
\hline $\begin{array}{l}\text { Series- } \\
\text { Parallel }\end{array}$ & $\begin{array}{l}\text { Better design freedom when com- } \\
\text { pared to parallel configuration } \\
\text { - Batteries can be re-charged in-flight }\end{array}$ & $\begin{array}{l}\text { Extra generator increases weight } \\
\text { - Complex control strategy } \\
\text { Engine not fully decoupled from pro- } \\
\text { peller }\end{array}$ \\
\hline $\begin{array}{l}\text { Partial } \\
\text { turbo- } \\
\text { electric }\end{array}$ & $\begin{array}{l}\text { Does not depend on advances of en- } \\
\text { ergy storage technologies } \\
\text { - Good design freedom for electric } \\
\text { motors-aircraft integration }\end{array}$ & $\begin{array}{l}\text { Depending on degree of hybridiza- } \\
\text { tion could be heavy and inefficient } \\
\text { - Gas turbine engine is not decoupled } \\
\text { from thrust generation }\end{array}$ \\
\hline
\end{tabular}





\section{4}

METHODOLOGY

To study and compare the impact that different propulsion system architectures have on the aircraft key performance indicators and overall design, it is first necessary to define a method that allows for the modelling of generic hybrid electric powertrains. This method needs to be flexible enough that at least all most discussed HEP architectures found in literature as presented in the previous chapter can be re-created.

A possible way to describe a HEP is through the use of power-based control parameters that describe the relative size in terms of nominal power of the electrical machines when compared to the total installed power. This power-based description has been already presented in the past for generalized dual-energy source systems $[52,53]$ that can be used to model unconventional powertrains where the relative size of the propulsive devices is kept homogeneous (i.e. the electric motors have all the same nominal shaft power).

Looking at the power-based control parameters, the methods described in literature can be differentiated between the ones that specify the hybridization at aircraft level and assume that it remains constant throughout the mission [54] and the ones that allow a large degree of controllability to the designer by assuming that the relative power ratios between electric and conventional can change along each mission segment $[55,56]$. The latter option further increases the complexity of the sizing method as more inputs are required to fully describe the problem but it is argued that the a more optimal design could be obtained with the more refined search of the design space. For this reason, the method developed for this study makes use of variable control parameters derived from user defined control laws defined at segment level. Moreover, differently from previously existing methods, the formulation for the sizing process allows also for the modelling of propulsive architectures making use of non-homogeneous (i.e. with different power ratings) propulsive units thus introducing an additional degree of freedom. The definition of controls and their segment specific law is given in section 4.1 .

The added complexity and larger design space deriving from the use of a hybrid propulsion systems requires a modification of well-established design methods to fully capture its impact on the aircraft design. The coexistence of multiple energy sources and power paths disrupts commonly used sizing routines as the result is highly influenced by the relative size of the power systems and their operation along the mission flight path.

The presence of a second energy source does not only have an impact on the number and type of required powertrain components and on the mission energy consumption but, depending on the architecture and degree of hybridization, it influences also the results of the constraint requirement analysis. The proposed sizing method, shown in figure 4.1, is therefore made up of three main modules: the constraint analysis, the point mass analysis and the aircraft/component sizing.

These modules require several inputs in the form of configuration parameters (e.g. number of propulsive devices, aerodynamic corrections due to aero-propulsive interaction and technology level of the components), top level requirements such as point performance constraints and mission requirements, and performance maps used to compute fuel flow and components efficiencies. As the latter inputs are not usually known at the conceptual design phase, semi-empirical and analytical procedures as described in section 4.2 are used 


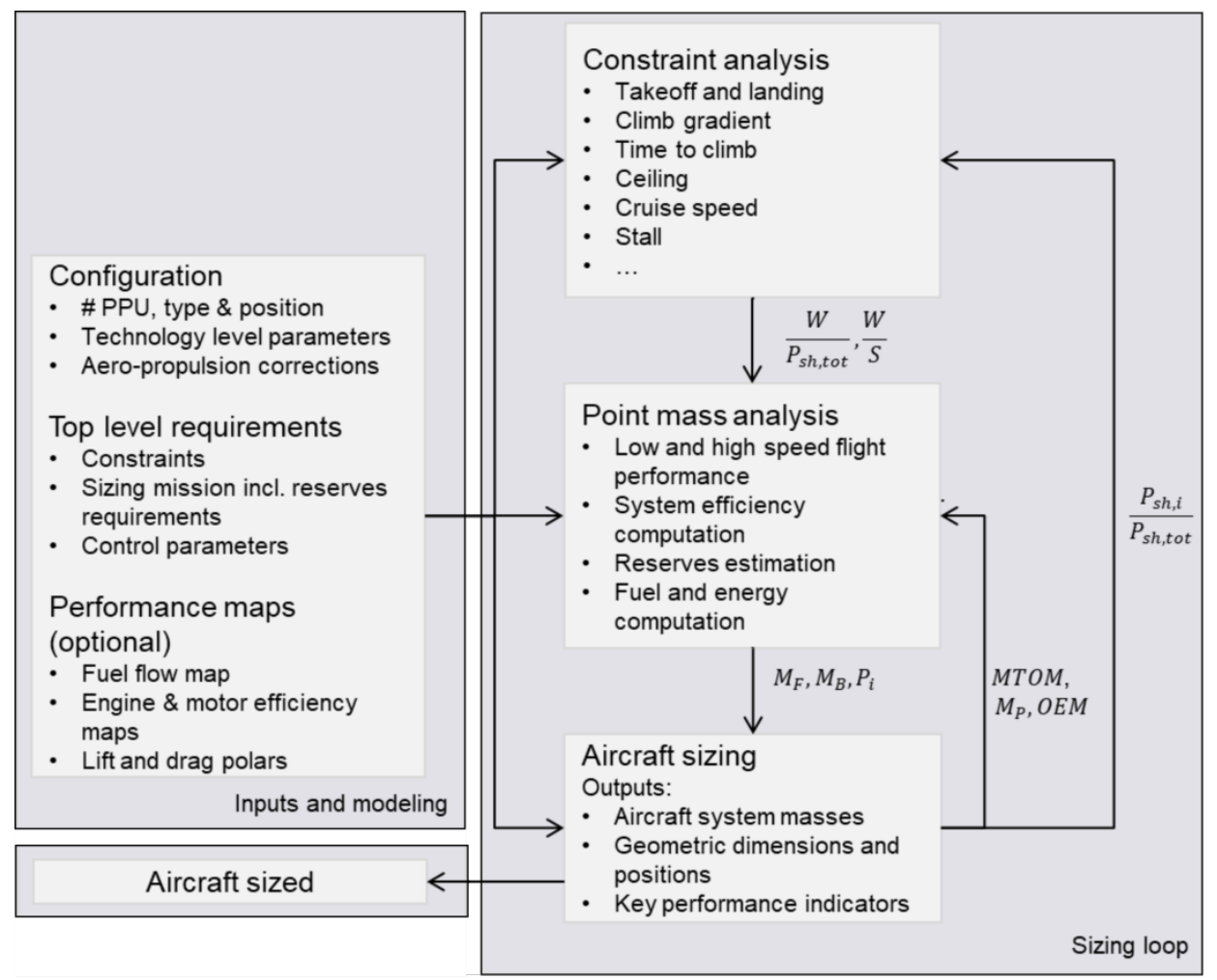

Figure 4.1: Flow chart of the proposed methodology for hybrid electric aircraft sizing.

to compute the missing information.

Looking at the three main modules, the constraint analysis computes the shaft power loading and wing loading required to satisfy the regulations and point performance requirements. The results are influenced by both the external (aero-propulsive) and internal definition of the propulsive system architecture as explained in section 4.3. As the method allows for differently powerful devices to co-exist in the same architecture, the shaft power loading is not directly used to size the powerplants as in other commonly used conceptual sizing methods [57]. Instead, the power to weight ratio is used as a starting point in the mission performance analysis.

The main objective of the point performance analysis is to compute the energy consumption as a function of the mission requirements. However, in the presented work it is also used to provide the information for the sizing of each object composing the propulsive system architecture. Using the segment specific control laws and component's efficiency rules, the computed propulsive power for a given point during the mission is propagated "upstream" all the way to the energy source following a concept of power path as presented in [56]. Once the power at the source is known, the energy requirement is computed through their time integration. This procedure is described in more detail in section 4.4 .

The maximum values computed in the mission analysis module at each component power-path location are corrected for flight condition (altitude, speed, air temperature) and then used in the sizing module to compute the weights and volumes of each propulsion system object. Finally, by adding these results together the aircraft weights (OEW, MTOW) are estimated. As the previous modules are affected by the overall masses and power ratios, the sizing loop is repeated until convergence is achieved and the design is fully sized.

\subsection{Propulsive Power Unit and control Parameters definition}

The proposed method aims to obtain a sizing procedure that enables the designer to obtain any of the propulsive system architectures discussed in chapter 3. To do so, the architectures possibility have been distilled down to a common object that, depending on the hybridization controls, can model any of the previously 
mentioned systems. This generic object has been named Propulsion Power Unit (PPU) and it is a collection of all the components that can be present in a hybrid electric powertrain. The number of PPUs in a conventional design is usually one for smaller general aviation aircraft and two to four for larger airplanes that require more redundancy for safety reasons. However, the upper limit for this number can be quite high as for example it is shown in distributed propulsion designs that utilize many small propellers to enhance the lift capabilities of the wing [58, 59].

\subsubsection{Propulsive Power Unit CONCEPT}

The PPU, showed in figure 4.2, is divided in two distinct sub-systems. The one on the left is exclusive to each PPU as it contains the propulsive device (P) such as propellers or fans, the mechanical gear-box (GB), and the power machines that provide the required shaft power, the gas-turbine (GT) and electric motor-generator (EMG). On the other side, there are the components that are shared between more PPUs such as the Power Management and Distribution system (PMAD), the electric generator (EG) and the energy reservoirs, fuel (F) and electro-chemical battery (B).

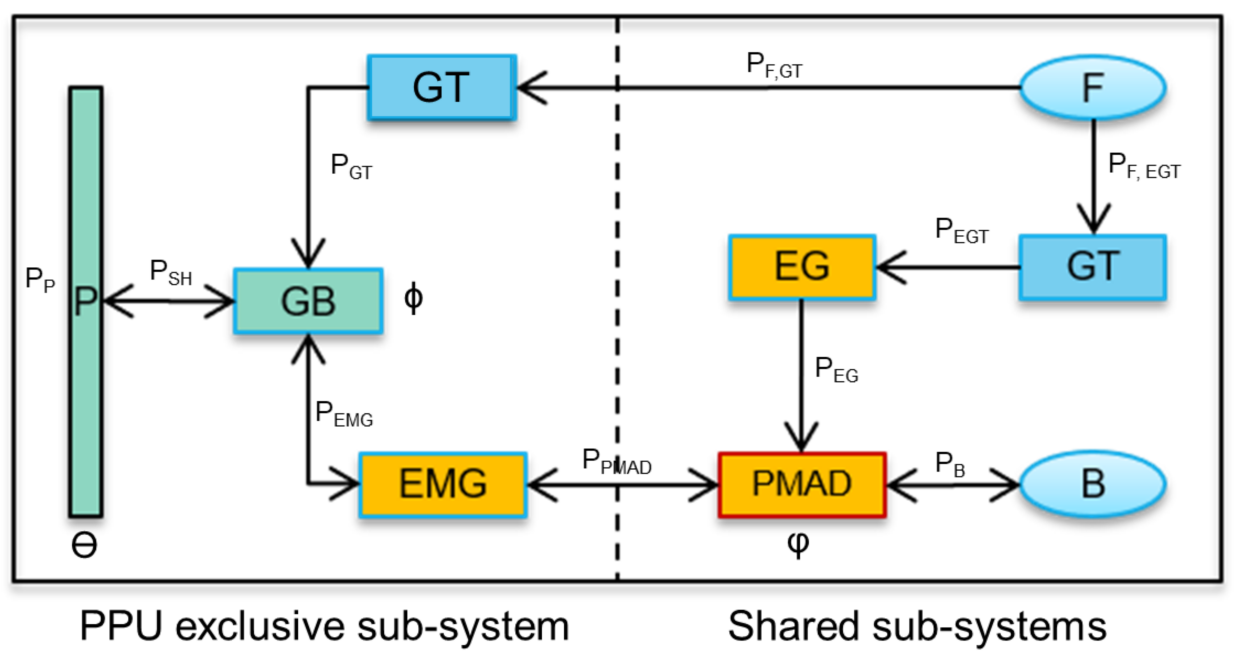

Figure 4.2: Schema of generic Propulsive Power Unit.

The distinction between the propulsive and system sides is required as it is expected that in a hybrid electric design there would not be as many fuel tanks, battery packs and generators as there are electric motors and gas-turbines. The system side components are sized by taking a given fraction of the sum of the power or energy requirements as computed in the mission module.

The direction of the arrows in figure 4.2 indicates the direction of the power flow along a power-path. It can be noticed that the electric power-path (propeller, motor-generator, PMAD and battery) offers the option of in-flight recharge of the battery either through a wind-milling propeller or by extracting excess power from the electric generator.

\subsubsection{CONTROL PARAMETERS}

To fully define the PPU power-paths one control at aircraft level and two at PPU level are used. The first parameter, the propulsive power share, is used to define the relative size of the PPUs through the ratio of the PPU propulsive power over the total propulsive power produced at the given instant in time as shown in figure 4.3:

$$
\Theta_{i}=\frac{P_{P, i}}{P_{P}}
$$

The bounds for this parameter usually vary between 0 and 0.5 for a twin-engine design as the two propulsors will provide up to half the required propulsive power each. If a propeller-motor system with regenerative 
capabilities is used, the control can be set to a negative value for part of the segment indicating that the propulsive device is being used to recharge the batteries. As this procedure decreases the total energy of the aircraft, it could be used to follow a steeper descent path or to off-set a powered-on approach as required from propulsion-enhanced high lift concepts (e.g. NASA X-57).

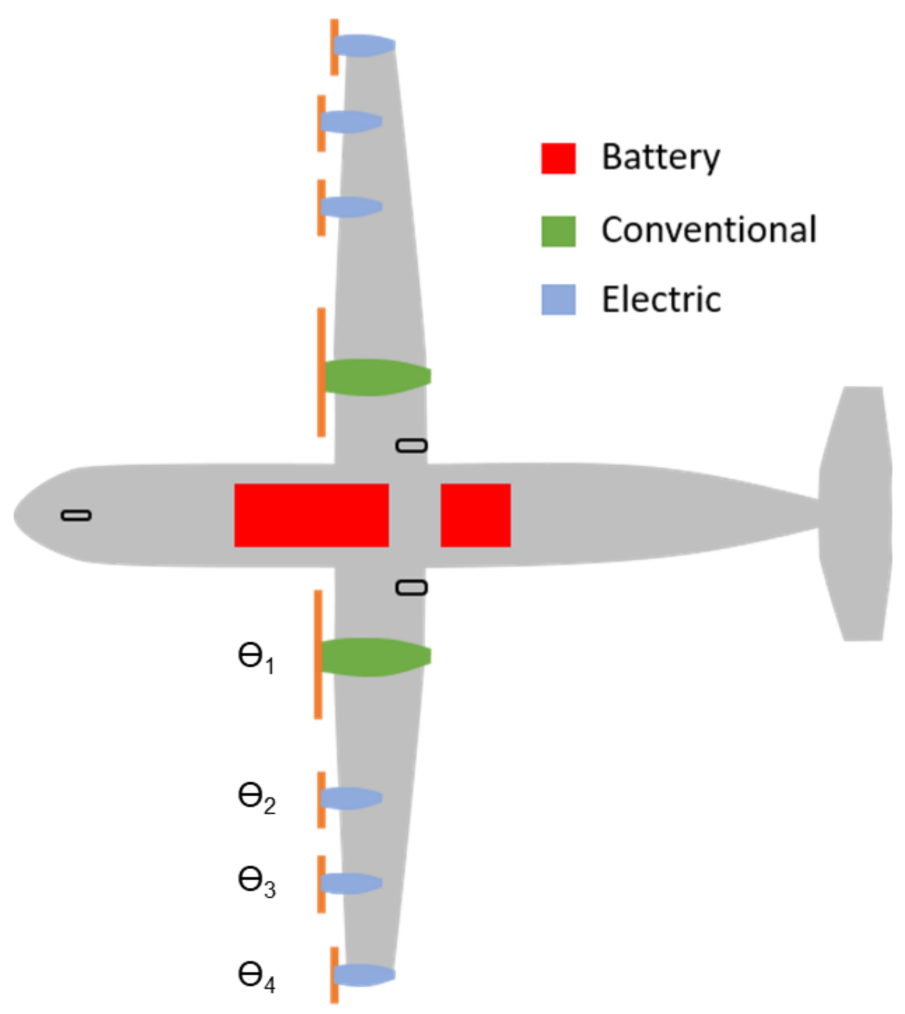

Figure 4.3: Propulsive power share used to differentiate between main (conventional) and auxiliary (electric) PPUs in a series-parallel architecture.

The remaining two control parameters are needed to solve the two power nodes present in each PPU. The first one is the shaft power ratio and it describes how much shaft power at the gearbox is generated electrically over the total instantaneous shaft power:

$$
\phi=\frac{P_{e m g}}{P_{\text {emg }}+P_{g t}}=\frac{P_{e m g}}{P_{\text {sh }, g b}}
$$

This control is constantly zero for fuel-based PPU and one for electrically driven propellers/fans. However, in the case of parallel architectures, it could vary along the mission in the interval $[0,1]$. As the electric motor is mechanically coupled to the gas-turbine engine, the range would be more limited to avoid compressor surge or other unwanted behaviours.

The final control parameter is the electric power ratio and it is needed to solve the electric node at the PMAD as it is assumed that in the presence of both a battery and an electric generator there would be a connection between the two electric buses. This ratio is defined as the instantaneous electric power provided by the battery over the sum of battery and generator power:

$$
\varphi=\frac{P_{b a t}}{P_{b a t}+P_{e g}}=\frac{P_{b a t}}{P_{P M A D}}
$$

This ratio is similar to the supplied power ratio $\Phi$ defined in [55] and [60] when the electric generator and battery efficiencies are considered: 


$$
\varphi=\frac{\eta_{b a t} P_{b_{s}}}{\eta_{b a t} P_{b a t_{s}}+\eta_{g t, s} \eta_{e g} P_{b a t_{s}}}=\frac{\eta_{b a t}\left(P_{b, s}+P_{f u e l, s}\right)}{\eta_{b a t} P_{b a t_{s}}+\eta_{g t, s} \eta_{\text {eg }} P_{b a t_{s}}} \Phi
$$

For turbo-electric architectures the parameters remain constantly equal to zero and conversely to one for designs that do not make use of generators such as the universally electric and parallel ones. For series architectures is can vary along the mission segments and when negative it indicates that the excess power produced by the generator-gas turbine system is being used to re-charge the batteries in flight. This could be used to shorten turn-around times in case of a fixed battery pack that cannot be easily replaced at the airport, albeit it would increase the fuel consumption and emissions generation.

As each PPU requires three parameters for its full definition, it would be time expensive to set up the tool for architectures using many PPUs such as the concepts of Distributed Electric Propulsion (DEP). However, it is expected that the PPU in such designs cab be aggregated by type so the tool allows the option to equate the controls of a PPU to another thus creating an "array of PPUs". In this way, the number of control laws that the designer needs to provide does not grow linearly with the number of PPUs but with the number of PPUs arrays instead (e.g. a conventional twin engine design needs only three control laws as the PPUs are symmetric and the same is valid for a DEP design using 10 PPUs).

\subsection{COMPONENTS SIZING AND EFFICIENCY}

In the sections below, the methods used in the sizing process of the HEP components are presented. Attention is given to the power conversion efficiency models as they are key in differentiating between propulsive system architectures. When the mass is not estimated directly from a technology assumption parameter (i.e. specific power and energy) the method used to evaluate the mass is also provided.

\subsubsection{PROPELLER}

Regardless of the mission segment that is being solved, the propagation of the power requirements through the propulsive power system starts with the evaluation of the required thrust power and its conversion to shaft power. To ensure that the point mass analysis is sensitive to the change of flight speed and altitude, a variable propulsive efficiency needs to be implemented in the tool.

The actuator disk method is chosen as the computation of the propeller efficiency is near instantaneous thus allowing its use in a conceptual design tool that needs to compute thousands of points for each mission. The propeller efficiency derived from the actuator disk theory is:

$$
\eta_{P, t h}=\frac{2}{1+\sqrt{1+\frac{T}{\frac{1}{2} \rho \frac{\pi}{4} D^{2} V^{2}}}}
$$

Where the subscript $t h$ denotes that this is the theoretical upper limit attainable as the disk actuator theory does not account for losses due to non-uniform axial velocities or rotational kinetic energy losses. For these reasons, the method is not accurate for low advance ratios typical of low speed, high power segments such as take-off. To use the propeller efficiency defined in equation 4.5 a correction factor is introduced. Sources report the theoretical propeller efficiency overestimates the actual efficiency by 10 to $15 \%$ in climb and cruise conditions thus the theoretical efficiency can be corrected with a constant value that can be calibrated depending on the propeller type.

For take-off conditions, the thrust might be limited by the maximum thrust coefficient. Reference [61] affirms that this can be considered using a limiting efficiency as shown in equation 4.6:

$$
\eta_{P, \text { limit }}=1.8 \rho B_{p r}^{0.15}\left(n D_{p r}\right)^{3.7} J P_{s h, 0}^{0.095} \cdot 10^{-7}
$$

Where $B_{p r}$ is the number of blades, $n$ is the propeller rotational speed in rev/s, $D_{P}$ the propeller diameter, $P_{s h, 0}$ the shaft power in $\mathrm{kW}$ and $\mathrm{J}$ is the advance ratio defined as the ratio between the flight speed and the product of propeller diameter and shaft rotational speed:

$$
J=\frac{V}{n D_{p r}}
$$


In constant speed propellers typical of turbo-prop engines, the propeller speed is kept constant throughout the mission and the propulsive power requirement is satisfied through the change of the blade pitch by an automatic governor. The propeller speed is limited by efficiency and noise requirements with a maximum Mach number at the tip, usually set at $M_{t i p}=0.7(250 \mathrm{~m} / \mathrm{s}$ at sea-level [62] $)$ but it can increase if highly swept propellers are used. As a first estimation, the flow velocity at the tip is function of the flight speed, the rotational speed and the propeller diameter:

$$
V_{t i p}=\sqrt{V^{2}+\left(\omega \frac{D_{p r}}{2}\right)^{2}}
$$

By rewriting this equation as a function of the maximum tip and flight Mach numbers, the rotational speed of the propeller in $\mathrm{rad} / \mathrm{s}$ becomes:

$$
\omega_{\max }=\frac{a}{\frac{1}{2} D_{p r}} \sqrt{M_{\text {tip }}^{2}-M^{2}}
$$

where $a$ is the sound speed at the considered flight altitude.

This method has the added benefit that the diameter and number of blades are the only required propeller geometry information. To fully parametrize this method and to make sure that the propeller changes alongside the aircraft dimension, the disk loading defined as the ratio between shaft power and propeller area is used as a design parameter. From equation 4.5 it can be deduced that a lower disk loading (i.e. larger propeller diameter for the same shaft power) increases the propulsive efficiency. However, the larger diameter also means that the propeller will become more massive as shown in the empirical equation 4.10 used to calculate the propeller mass [62]:

$$
m_{p r}=0.124 N_{p r}\left(D_{p r} \sqrt{B_{p r}} P_{s h, 0}\right)^{0.78174}
$$

where $N_{p r}$ is the number of propellers on each shaft.

\subsubsection{GEARBOX}

Even the more advanced propellers that use a large number of swept blades have rotational speeds that are limited when compared the optimal turbine speed of turbo-shaft engines that are around an order of magnitude larger. Similarly, electric machines size decreases with shaft speed so high rotational speed are beneficial to the mass and volume of electric motors and generators.

A gearbox is therefore required to mechanically couple the propeller to the shaft power source. Reference [63] provides a mass correlation that depends on the gearbox nominal power and the reduction speed ratio:

$$
m_{g b}=k_{g b} \cdot \frac{\left(P_{g b}\right)^{0.76} \cdot\left(n_{\text {in }}\right)^{0.13}}{\left(n_{\text {out }}\right)^{0.89}}
$$

where the power is provided in $\mathrm{kW}$, the rotational speeds in RPM and the result is in $\mathrm{kg} . k_{g b}$ is a technology factor used to correct the mass accounting for future technology improvements. Reference [64] suggests values of 34 for modern gearboxes and 26 for future applications.

Gearbox for high power, high speed applications as the one required in the aviation sector make use of epicycloid gearing that remain highly efficient throughout the speed and power envelope. The study of reference [65] shows that for a fixed shaft speed, the friction losses in the gearbox are a function of the power. Through a power regression of the study results, the correlation of equation 4.12 is obtained.

$$
\eta_{g b}=99.45-\frac{0.6771 \cdot P_{g b}}{P_{s h}}
$$

The method has a correlation coefficient $R$ of 0.998 but it needs a lower bound for the power fraction to avoid negative values when the required power nears zero. This is set at $P_{s h} / P_{g b}=0.01$ (i.e. $\eta_{g b} \tilde{=} 32 \%$ ). Figure 4.4 shows the power regression curve and the results of study [65]. 


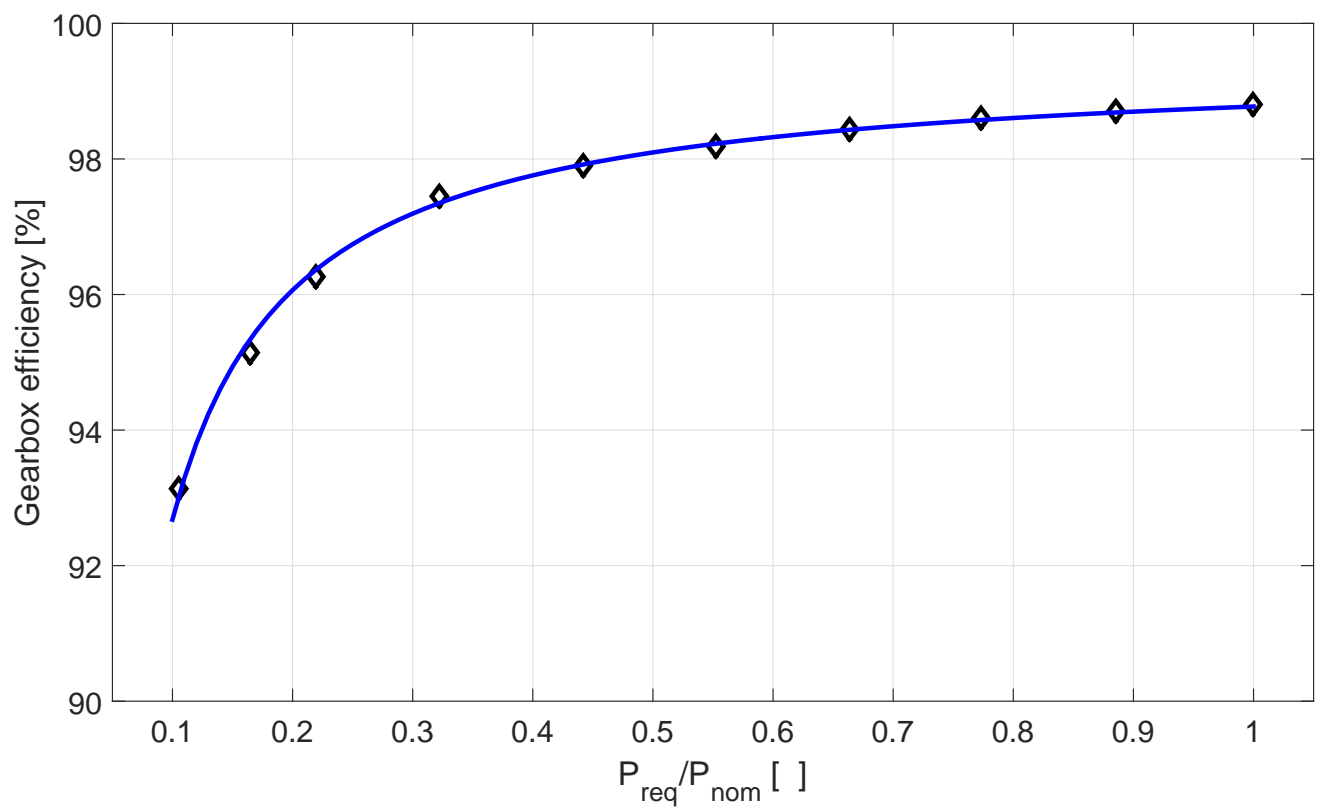

Figure 4.4: Gearbox efficiency results of reference [65] and power regression curve.

\subsubsection{TURBO-SHAFT ENGINE}

The turbo-shaft engine component in this study is used to provide mechanical power to the propeller or to an electric generator. The dry mass of the engine is related to its shaft power as suggested by [66]. By using the engine values collected by Meier $^{1}$, a power regression curve is obtained described by equation:

$$
m_{g t}=0.9594 \cdot k_{g t} \cdot P_{s h, g t}^{0.7976}
$$

where the factor $k_{g t}$ can be used to account for expected improvement in turbo-machinery technology.

Figure 4.5 shows the engine data with its regression curve in blue and the results of Raymer's regression curve in red [66]. For shaft powers between 200 and $1500 \mathrm{~kW}$ the regression curve approximates the data well while the increasing spread from $1500 \mathrm{~kW}$ onwards indicates that this curve is not as reliable for larger machines. Nevertheless, the curve is used as no better methods that could be used to estimate the engine mass at a conceptual design level could be found.

Turbo-shaft engine efficiency is not directly assessed. Instead, a fuel flow map normalized by the engine nominal shaft power is used to first compute the fuel flow as a function of flight state (altitude and Mach number) and throttle Consequently the efficiency can be computed as the ratio between shaft power and fuel power (product of fuel flow and fuel specific energy):

$$
\eta_{g t}=\frac{P_{g t}}{\epsilon_{\text {fuel }} \dot{m}_{\text {fuel }}}
$$

\subsubsection{ELECTRIC MOTOR AND GENERATOR}

At a low level of detail electric motors and generators can be modelled in the same way as they are based on the same working principles. In this section only the electric motor characteristics will be presented but the same are valid for generators by considering that input and output power directions are reversed.

An electric motor is a machine that transforms electric power into mechanical power. The shaft power produced by an electric motor is defined by the product of its shaft speed and torque:

$$
P_{\text {em }}=\omega Q
$$

\footnotetext{
${ }^{1}$ Nathan Meier. Jet engine specification database. http://www.jet-engine.net, 2005. Accessed on 19/02/2018.
} 


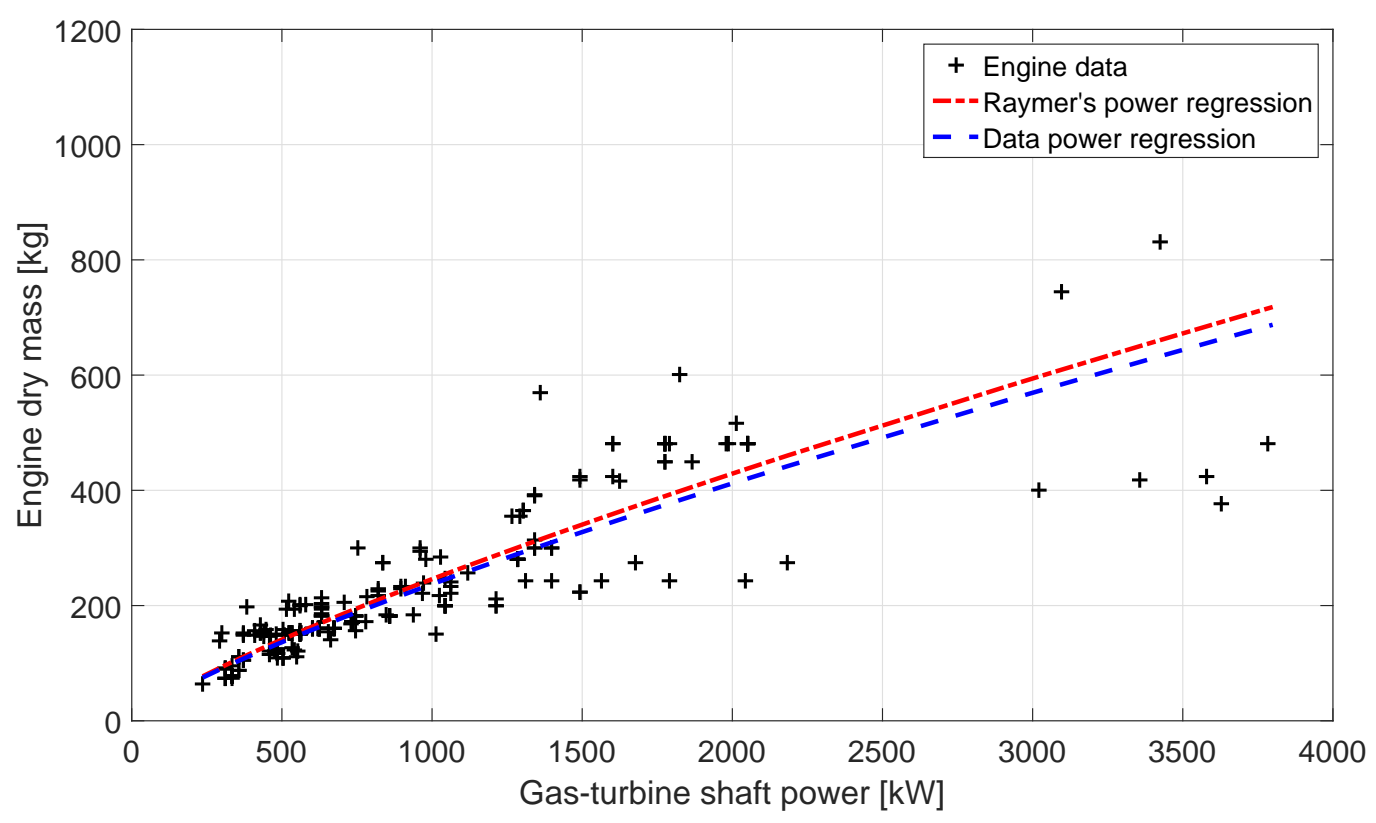

Figure 4.5: Regression curve of turboprop data and comparison with result from [66]

these two values are function of the electric input power and motor characteristics. The torque that the motor can produce is directly proportional on the electric current, I, the magnetic flux, $\Phi_{b}$, and on several motor aspects (e.g. number of pole pairs, turns for each coil etc.) grouped in the parameter $k_{e m}$ that, in first approximation, can be considered constant [67]. As the magnetic flux is usually generated by permanent magnets, it can also be considered constant and grouped with $k_{e m}$ in the constant $k_{I}$ :

$$
Q=k_{e m} \Phi_{b} I=k_{I} I
$$

When conductive wires move in a magnetic field, an electric potential is generated. This voltage, $U_{\text {bemf }}$, is usually called back EMF (Electro-Motive Force) as it is in opposition to the supplied motor voltage, $U_{e m, s}$. The magnitude of the back EMF in a rotating coil depends on the magnetic field strength, the dimensions of the coil (number of wire turns, length, radius) and most importantly on the rotational speed, $\omega$. The electric current passing through the motor coils is function of the net voltage applied to the armature and of the coil internal resistance. As the net voltage results from the difference between supply voltage and back EMF, the current becomes [67]:

$$
I=\frac{\Delta U}{R_{a}}=\frac{U_{e m, s}-U_{b e m f}}{R_{e m, i}}=\frac{U_{e m, s}}{R_{a}}-\frac{k_{I}}{R_{e m, i}} \omega
$$

By substituting equation 4.17 into equation 4.16, it results that the motor torque decreases linearly for a fixed magnetic flux intensity. The motor speed can easily be modulated through the change of the supplied voltage or, if electro-magnets are used instead of permanent ones, through the change of magnetic flux.

Practical limits exist that restrict the torque-speed characteristics of an electric motor as shown in the envelope of figure 4.6. In the first region between shaft speed 0 RPM and $\omega_{\text {rated }}$, the maximum current cannot achieve the value of $\frac{U_{e m, s}}{R_{e m, i}}$ as this would imply excessively high currents that could damage the motor [68]. Therefore, the torque is kept constant up to the point at which the back EMF limits the current that can be drawn from the supply. This point is the used to size the electric motor and it is characterized by the equation:

$$
P_{\text {rated }}=\omega_{\text {rated }} Q_{\text {rated }}
$$

Further increasing the motor shaft speed is possible but due to the increasing back EMF, the torque will decrease more rapidly. This limit is well approximated by a constant power curve [68]. This curve remains valid up to the maximum shaft speed, $\omega_{\text {limit }}$, at which point losses due to speed become predominant and the 


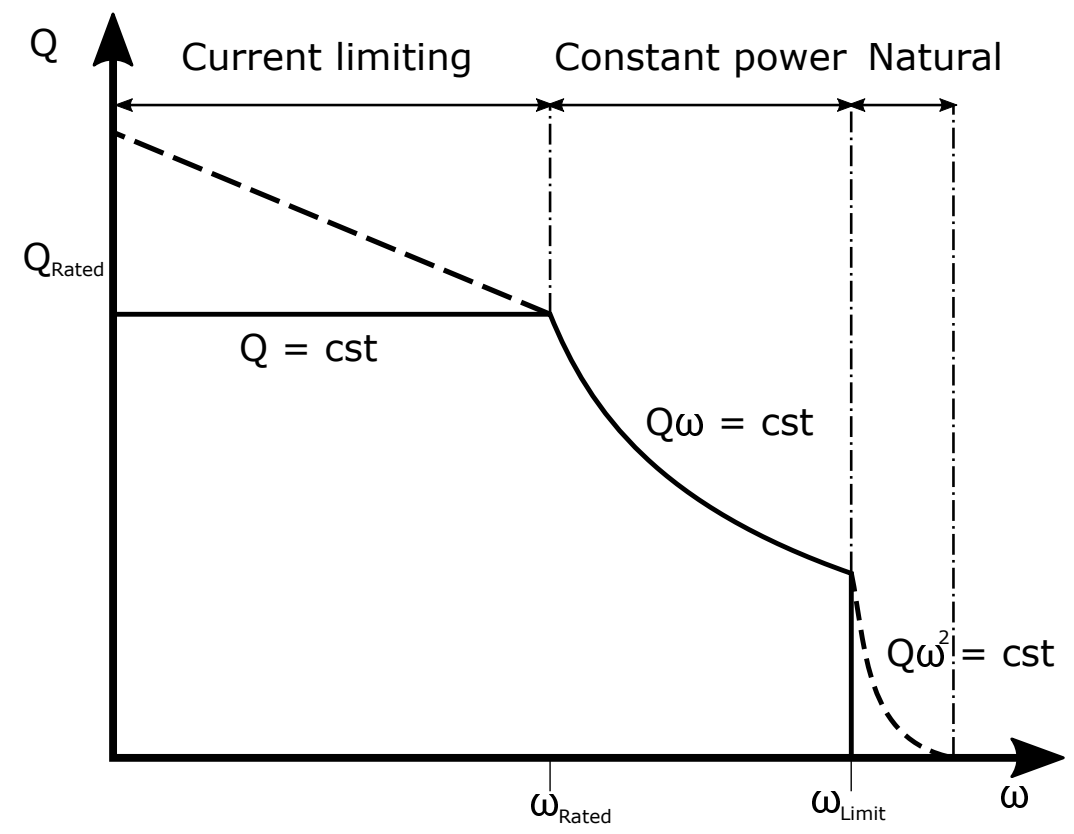

Figure 4.6: Electric motor map.

usable torque rapidly falls. The operation in this region is possible but of low interest as it is characterized by high losses and very low torque values.

The source of power loss in an electric motor can be grouped into four categories as described in [67]:

- Ohmic losses: these losses, also called copper losses, are caused by turning part of the supplied electric power into heat energy when electric current flows through a resistance as defined in equation 4.29. As the motor torque depends on the armature electric current, these losses are proportional to the square of the shaft torque: copper losses $=k_{\text {copper }} Q^{2}$.

- Core losses: also known as iron losses, are dependent on two phenomena that are developed when a conductive material is subjected to a variable magnetic field as it is the case for the cyclically changing magnetic field seen by a rotating coil. The first loss is due to the energy required to continually magnetise and demagnetise the material. As the material dipoles alignment lags behind the magnetic field orientation (hysteresis), energy is lost at each cycle. The second loss is due to the currents induced in the conductive material as the magnetic field changes. As the currents rotates in the material, these losses are called "eddy current losses". Both the hysteresis and eddy current losses directly depend on the frequency at which the magnetic field changes, or in other words, on the rotational speed of the coils: iron losses $=k_{\text {iron }} \omega$.

- Friction losses: these losses will be experienced by the rotor as shaft power is produced. These losses depend on the mechanical friction in the bearings and on the air-drag experience by the rotating components. This inefficiency grows with the cube of the rotational speed: friction losses $=k_{f} \omega^{3}$.

- Constant losses: these are losses that do not depend on the rotational speed or torque required from the motor. Examples of these are the power required to excite the electro-magnets or for the electronic circus that are required to control the machine. These losses are denoted with the letter $\mathrm{C}$.

By combining all the sources of energy loss as described above, we obtain:

$$
P_{\text {loss }}=k_{\text {copper }} Q^{2}+k_{\text {iron }} \omega+k_{f} \omega^{3}+C
$$

by then defining the motor efficiency as the ratio between output and input power we have:

$$
\eta_{\text {em }}=\frac{P_{\text {em }}}{P_{\text {em }}+P_{\text {loss }}}=\frac{\omega Q}{\omega Q+P_{\text {loss }}}=\frac{\omega Q}{\omega Q+k_{\text {copper }} Q^{2}+k_{\text {iron }} \omega+k_{f} \omega^{3}+C}
$$


The coefficients required to compute the power loss depend on the motor type and internal configuration. This means that directly computing the coefficients would be possible if characteristics of the electric machine such as number of poles, coil windings, bearing, rotor diameter and others are known a priori.

However, this makes the method non-scalable and difficult to use for large aircraft design space explorations. The study of reference [68] presents a solution to this problem by describing the losses coefficients as functions of four parameters that are independent from the internal characteristics of the motor. These are the optimum efficiency, torque and shaft speed, $\hat{\eta}, \hat{Q}, \hat{\omega}$ and the parasitic (i.e. constant) loss ratio, $k_{0}$. The losses coefficients become:

$$
\begin{gathered}
k_{\text {copper }}=\frac{\hat{\omega}(1-\hat{\eta})}{2 \hat{Q} \hat{\eta}} \\
k_{\text {iron }}=\frac{-3 C_{0}}{2 \hat{\omega}}+\frac{\hat{Q}(1-\hat{\eta})}{4 \hat{\eta}} \\
k_{f}=\frac{C_{0}}{2 \hat{\omega}^{3}}+\frac{\hat{Q}(1-\hat{\eta})}{4 \hat{\eta} \hat{\omega}^{2}} \\
C=k_{0} \frac{\hat{\omega} \hat{Q}}{6} \frac{1-\hat{\eta}}{\hat{\eta}}
\end{gathered}
$$

Three additional coefficients, $k_{\omega}, k_{P}, k_{Q}$, are required to determine the limits of the torque-speed envelope and define the optimum values as function of the rating parameter:

$$
\begin{gathered}
P_{\text {rated }}=k_{P} \hat{P}=k_{P} \hat{\omega} \hat{Q} \\
Q_{\text {rated }}=k_{Q} \hat{Q} \\
\omega_{\text {rated }}=\frac{k_{P}}{k_{Q}} \hat{\omega} \\
\omega_{\text {limit }}=k_{\omega} \hat{\omega}
\end{gathered}
$$

With these seven parameters the power loss characteristics of a given motor are completely defined. The result of a sample $150 \mathrm{~kW}$ motor is shown in figure 4.7. It can be noticed that electric machine is characterized by a very high-power conversion efficiency throughout the working envelope. The efficiency quickly falls only for low speed or no-load operations where the power requirement is so limited that there is no impact on the sizing of the rest of the system.

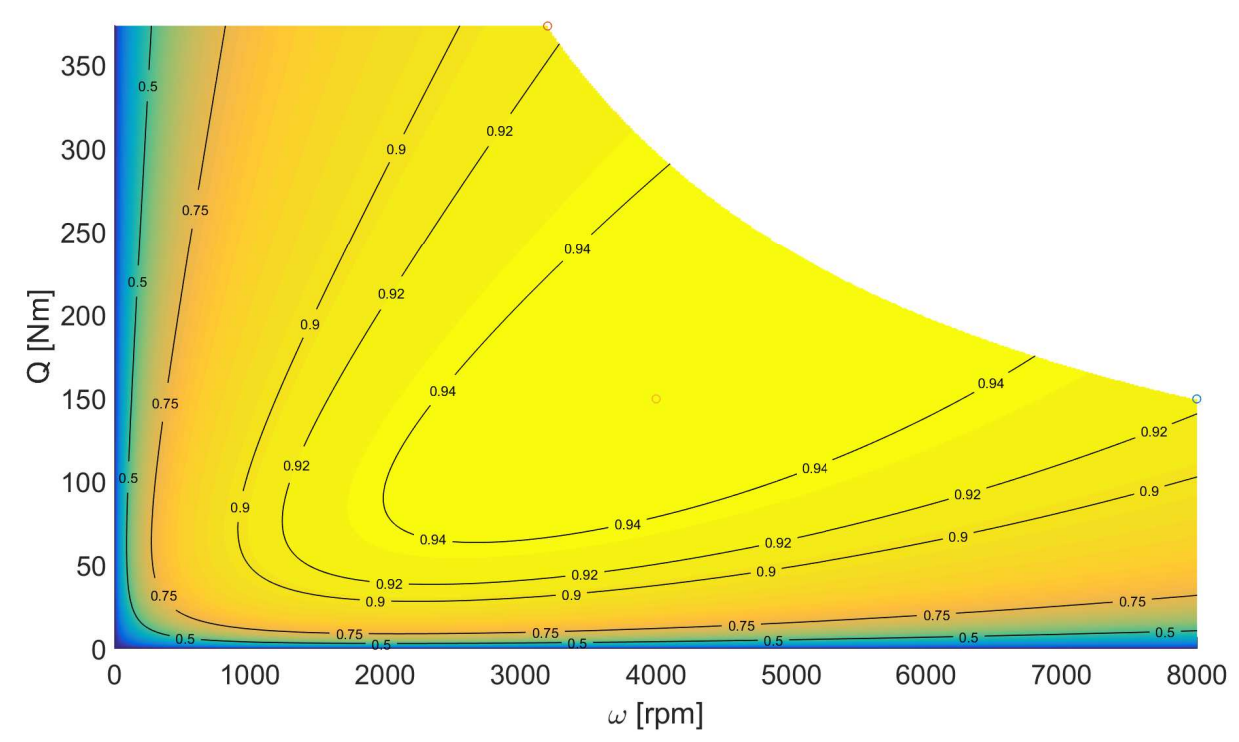

Figure 4.7: Electric motor characteristics with iso-efficiency lines. 
The mass of the electric machines is calculated using the specific power parameter. The scalability of electric motors encourages the use of this assumption. However, it exists a maximum power rating at which the assumption of constant specific power with increasing motor dimension is not valid any more. As an electric machine is scaled up with increasing power, the ratio between external surface and internal volume decreases making the cooling of the machine more difficult [64]. After a certain threshold, the heat management requirements become critical in the sizing of a motor. To solve this issue, either a more complex cooling system is installed, or the current density of the windings is reduced. Both options increase the motor mass, so the gravimetric power of an electric machine should increase with the value of its maximum continuous power. This effect is not modelled in this study as it would require methods to find the size at which heat production and dissipation reach an equilibrium making it too complex for a conceptual design tool. Nevertheless, it should be kept in mind that distributing the power requirement over more electric motors could be a more feasible solution for designs with high degree of hybridization (e.g. universally electric, series architectures).

\subsubsection{HigH-POWER CABLING SYSTEM}

Cables are necessary for the transmission of electric power between the components of the HEP. In a DC cable, the power transmitted is proportional to the product of the system voltage, $U$, and the electric current, I, while the losses grow with the square of I as determined in Ohm's law:

$$
P_{\text {co,loss }}=I^{2} R_{\text {co }}
$$

The heat generated in this way limits the minimum area of the cable as the resistance grows with its inverse. This limit is captured with the current area density, $\mathrm{j}$ defined as the maximum electric current that can be transmitted through a cable conductor of section $A_{c o}$ :

$$
j=\frac{I}{A_{c o}}
$$

As the resistance and heat dissipation characteristics depends on the conductor material and isolation, the current density can be used as design variable.

Once the cable area is known, the length specific weight, $w_{c b}^{*}$, is computed as a function of the conductor, isolation and sheath materials' volumetric densities as explained in [64]. The isolation is required to avoid short circuits with other cables or the surrounding environment. These are due to the difference in electric potential so the isolation material thickness, and consequently its weight, are a function of the system voltage. The sheath is used for protection against physical damage, so its thickness is function of the cable diameter [64].

$$
w_{c b}^{*}=w_{c o}^{*}+w_{i s o}^{*}+w_{\text {sheath }}^{*}
$$

Typical materials used in conventional cables are copper and aluminium. The first offers low resistance at the cost of a higher density while the latter is lighter but due to higher losses it is limited to lower current densities. These values are reported in table 4.1. The length specific resistance of the conductor is function of its section and specific resistivity, $\sigma$ measured in $\Omega \mathrm{mm}^{2} / \mathrm{m}$ :

$$
R_{c o}^{*}=\frac{\sigma_{c o}}{A_{c o}}
$$

The resistivity of a metal conductor increases with temperature. This temperature is affected by the heat generated due to Ohmic losses and the heat dissipation of the cable. Depending on the power rating of the cable, the heat dissipation can be passive or improved with the use of coolant pumped in a sleeve surrounding the cable. Heat balance calculation and temperature transients during the mission are not modelled in this study so, to consider the impact of temperature on the resistivity, the values at $90{ }^{\circ} \mathrm{C}$ (maximum operating temperature of the insulator) are used for copper or aluminium conductors as reported in table 4.1.

The length of the cable is automatically estimated depending on the relative distance of the components that need to be connected. These are the inverters of electric motors to the PMAD and the connection between the modulation sub-system and the power producers (battery packs and electric generators). For each of these cables a system voltage can be defined alongside the cable materials proprieties. Moreover, a DC connection 


\begin{tabular}{lccc}
\hline & & Copper & Aluminium \\
\hline$\rho_{c o}$ & {$\left[\mathrm{~g} / \mathrm{cm}^{3}\right]$} & 8.9 & 3.3 \\
$\sigma_{c o, 20^{\circ} \mathrm{C}}$ & {$\left[\frac{\Omega \mathrm{mm}^{2}}{\mathrm{~m}}\right]$} & $1.8 \cdot 10^{-2}$ & $2.9 \cdot 10^{-2}$ \\
$\sigma_{c o, 90^{\circ} \mathrm{C}}$ & $2.3 \cdot 10^{-2}$ & $3.7 \cdot 10^{-2}$ \\
\hline & & Insulation and Sheath \\
\hline$\rho_{\text {iso }}$ & {$\left[\mathrm{g} / \mathrm{cm}^{3}\right]$} & \multicolumn{2}{c}{1.3} \\
$\rho_{\text {sheath }}$ & {$\left[\mathrm{g} / \mathrm{cm}^{3}\right]$} & \multicolumn{2}{c}{1.5} \\
\hline
\end{tabular}

Table 4.1: Material characteristics of conventional cables.

requires a positive and negative pole, so each connection is multiplied by two. As these cables provide the power required for propulsive purposes, they are considered critical components so a further multiplication factor, $k_{r d}$ (usually set at two) is used to compute the total weight of a line. Figure 4.8 shows the cabling paths along the wing used to estimate cable length, $l_{c b}$.

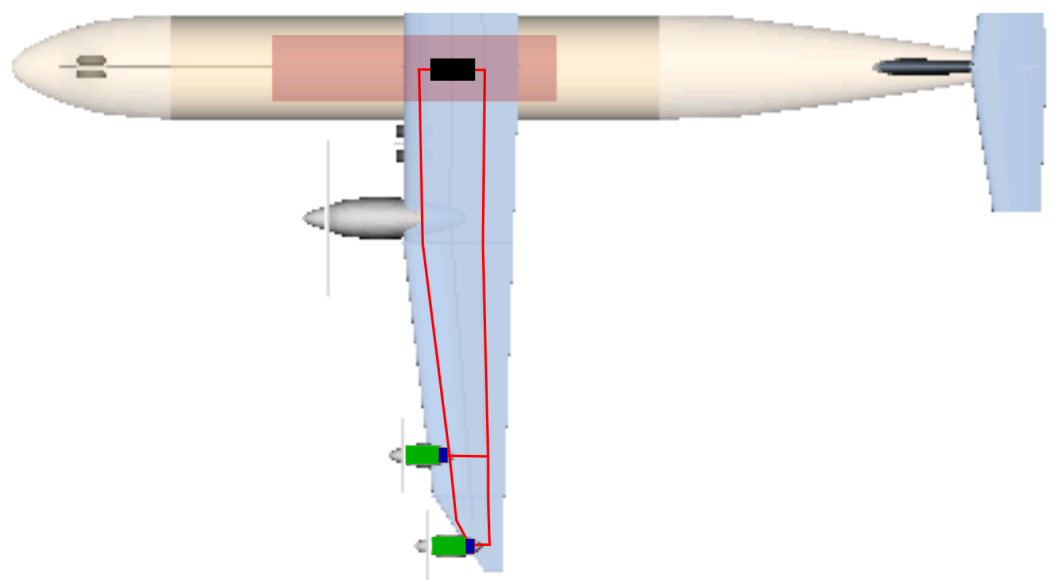

Figure 4.8: Cabling paths (red) along wing span connecting motor inverters (green) to the PMAD sub-system (black).

With the cable resistance known, the efficiency of the power transmission is computed as a function of the power requirement at a given instant:

$$
\eta_{c b}=\frac{P_{\text {out }}}{P_{\text {out }}+P_{\text {co,loss }}}=\frac{\frac{I}{k_{r d}} U}{\frac{I}{k_{r d}} U+\left(\frac{I}{k_{r d}}\right)^{2} R_{c o}}=\frac{1}{1+\frac{1}{k_{r d}} \frac{I \cdot R_{c o}}{U}}
$$

Since during normal operations each cable line can be used to provide power, losses can be reduced by keeping a constant system voltage and splitting the electric current in as many parallel legs as there are lines instead of using only one line. This is accounted in the efficiency computation with the line redundancy factor $k_{r d}$.

\section{High Temperature Superconducting CABLES}

As seen in chapter 2.2.2, HTS cables could be an interesting technology for electric power distribution as they offer lossless transmission and their very high current density could translate into weight savings. The main drawback is that, to keep the conductor material below its critical temperature, a cryogenic cooling system is required increasing weight and complexity.

In a superconducting DC cable there are no ohmic losses. The mass estimation method applied for conventional electricity transmission based on the maximum current density is not applicable any more as there is no clear way to correlate the cable cross section to the power losses. Moreover, the weight of an HTS cable is dominated by the non-conductive materials such as the insulator, cryo coolant and protective sheath layers [64]. For these reasons the length specific mass of the superconducting cable is assumed as non-influenced by voltage and current changes and set at a constant value of $5 \mathrm{~kg} / \mathrm{m}$ as reported in [69, 70]. 
Although negligible heat is produced by the conductor, the imperfect insulation and losses at the cable terminals require that the transmission is actively cooled through the removal of heat by a cryo-cooler machine. Reference [69] indicates that the heat generation due to defects in the insulation can be modelled as a length specific power loss of $5 \mathrm{~W} / \mathrm{m}$ while reference [71] states that each cable terminal introduces an amount of heat dependent on the electric current in $\mathrm{kA}$ and number of connecting leads per cable (usually 2) as defined in equation 4.34:

$$
P_{\text {loss, terminal }}=45 N_{\text {leads }} I+200[W]
$$

The total heat power that needs to be extracted from the cryo-coolant is therefore a function of the electric current and by consequence of the instantaneous electric power requirement. The maximum value found during the point mass analysis will be then used as a sizing requirement for the cryo-cooler.

\subsubsection{Electric Power Management and Distribution sub-system}

The PMAD in this method is used as a simplification of the electric sub-system required to distribute and modulate the electric power. Electric power can be supplied as direct or alternating current and at different voltage levels. However, for each of these conversions part of the power is lost in the form of heat as the electric components are not perfectly efficient. As a simplification, it is assumed that all the electric components connected in a PMAD are characterized by the same efficiency behaviour as more or less power is drawn from the electric sources. Reference [4] suggests that by making this assumption, the overall efficiency of the PMAD becomes the product of the $N$ electric components connected in series:

$$
\eta_{P M A D}=\prod_{i=1}^{N_{e c}} \eta_{e c, i}=\eta_{e c}^{N_{e c}}
$$

The number of components in the PMAD is a design variable and it depends on the type of the electric power internal architecture. As presented in the qualitative study of chapter 3 , the number of components could go from zero for a turbo-electric HEP architecture run completely in AC and with a fixed ratio between motor and generator shafts speeds, to three or more for HEP using both generators and batteries as sources of electric power as a rectifier, a transformer and an inverter would be necessary at the minimum.

Constant conversion efficiencies in the range of $95 \%$ for conventional electric components are assumed in this study. In superconducting technologies are used, conversion as high as $99.8 \%$ are considered achievable [72]. The mass of the PMAD sub-system can be derived from the number of expected components and the average specific power:

$$
m_{P M A D}=\sum_{i=1}^{N_{e c}}\left(\frac{P_{P M A D}}{\hat{\rho}_{e c, i}}\right)=N_{e c} \frac{P_{P M A D}}{\hat{\rho}_{e c, i}}
$$

Values for the gravimetric specific power of electric components, both conventional and super-conducting, are reported in the background information presented in chapter 2.2.

\subsubsection{BATTERY}

Electro-chemical batteries are one of the two sources of electric power considered in this work alongside electric generators. A battery is defined as a system of two or more electric cells that convert chemical energy into electric energy. The fundamental block of the battery, the cell, is composed by a positively and negatively charged electrodes joined by an electrolyte. When the two electrodes are connected to a circuit, the electric potential between the two elements generates an electric DC current [67].

The energy conversion process inside a cell is not perfectly efficient as part of the power produced is lost due to ohmic, activation and concentration losses that depend on the cell State Of Charge, SOC, and battery current, $I_{b a t}$ [73]. The usable voltage of a cell can therefore be described by equation 4.37 where $U_{c, o c}$ is the open-circuit voltage of the cell and $R_{c}$ its total internal resistance:

$$
U_{c}=U_{c, o c}-R_{c} I
$$

This simple model of a battery cell, as shown in figure 4.9, can be used to determine several of the battery characteristics if the number of cells and their configuration in the battery pack are known. 

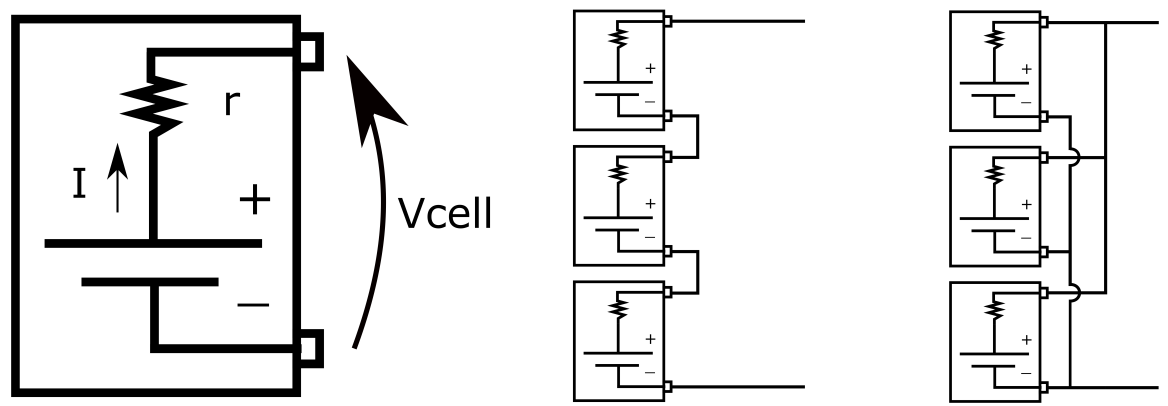

Figure 4.9: Electrochemical cell model, series and parallel battery connections.

Both the cell open-circuit voltage and internal resistance depends on several factors such as for example the cell remaining capacity and the environment temperature. This makes realistic battery modelling very difficult, so simplified models need to be used for obtaining results in low order design approaches. Cinar [17] and Vratny [74] present two different methods to obtain the characteristic discharge curve of lithiumbased batteries.

An example of a cell discharge curve from [17] is shown in figure 4.10 where the cell voltage changes as the cell is discharged. If the cell is discharged at a higher rate (i.e. higher current) the curve shifts downward following equation 4.37. The method presented in [75] and used by Vratny obtains similar results but instead of defining points on the discharge curve as [17] does, it uses experimentally derived functions for the opencircuit voltage and internal resistance. Both methods neglect temperature dependencies and self-discharge effects. Moreover, the method proposed by Cinar further assumes a constant internal resistance (i.e. it does not account for concentration losses).

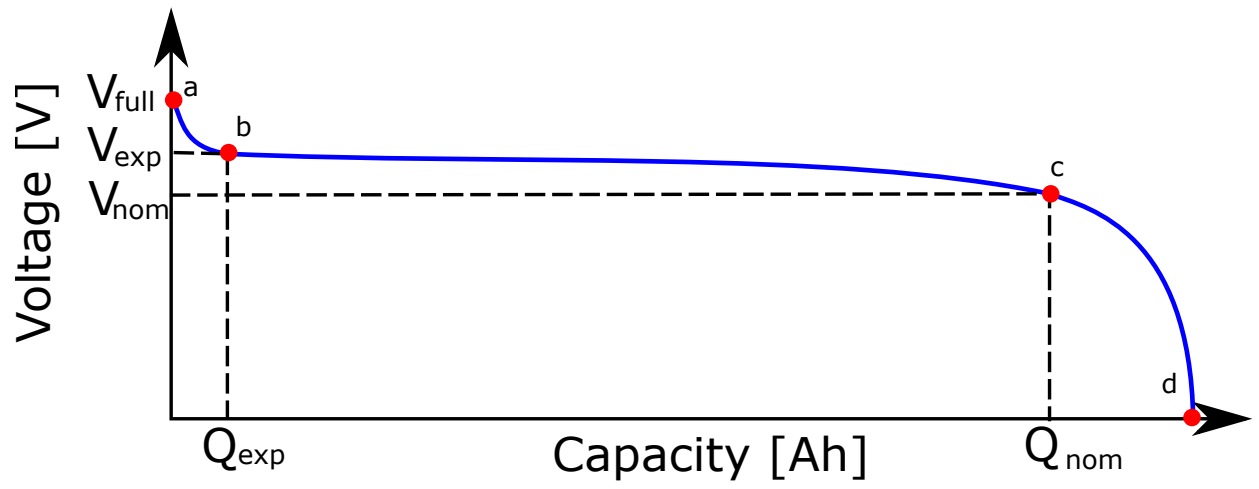

Figure 4.10: Sample discharge curve at fixed discharge rate adapted from [17]

The issue of both methods is that they require the knowledge of the cell chemistry and battery proprieties to solve the system making them difficult to implement in a conceptual design method. Instead, in this study a simplified approach is proposed.

The first assumption behind this new approach is that the highly variable voltage drops between points $a-b$ and c- $d$ of the curve in figure 4.10 can be omitted. This assumption is based on the fact that the exponential segment ( $a-b)$ is usually negligibly small for lithium-based cells and the cell should not be used after point $c$ to avoid permanently damaging the cell. In this way, the concentration losses are negligible and the voltage drop between fully charged and discharged cell can be assumed linear.

The second assumption is that the power required at the battery changes slowly enough that the highly dynamic effects can be omitted. In this way also the activation losses can also be neglected thus allowing the use of a constant cell resistance as in the study of reference [17]. Therefore, the entire cell and battery characteristic can be described with the open-circuit voltages for a fully charged and discharged cell, its internal resistance and the electric system power and voltage requirements. The discharged cell voltage, also called 
cut-off voltage, can be defined as a fraction of the open-circuit fully charged cell voltage as the ratio remains constant for different discharge rates [64].

Figure 4.9 shows the two ways in which electric cells can be connected to build a battery. When connecting cells in series (i.e. connecting opposed charged electrodes), the voltages and resistances of the cells are added together to get the equivalent circuit. A series of cells connected in this way is called a battery module [74]. By defining the electric system voltage and the cell cut-off voltage the required number of cells in series becomes:

$$
N_{\text {ser }}=\frac{U_{s}}{U_{c, \text { cutoff }}}=\frac{U_{s}}{k_{\text {cutoff }} U_{c, o c}}
$$

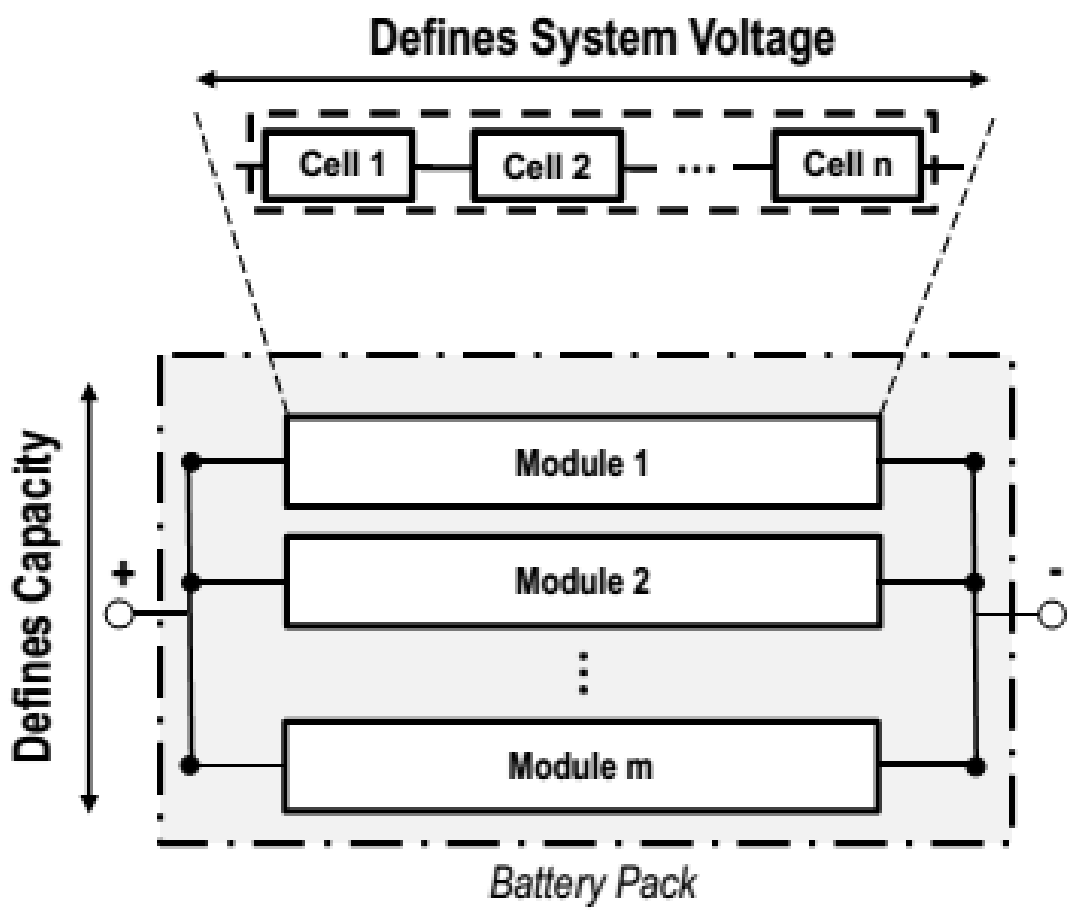

Figure 4.11: Battery pack model [74]

Using equation 4.37 and depending on the cell internal resistance, the current at which the cell voltage equals the cut-off potential is:

$$
I_{c, \max }=\frac{U_{c, o c}-U_{c, \text { cutoff }}}{R_{c}}
$$

This is the maximum current admissible at cell level and it can be used to compute the number of battery modules required to supply the battery rating power. Connecting modules in parallel (i.e. connecting electrodes with the same sign together) also sums the capacity of the modules together as indicated in figure 4.11. Therefore, the number of modules in parallel, $N_{p}$, needs to be the maximum value between the power and energy requirements, $N_{\text {par,I }}$ and $N_{\text {par, }}$ respectively:

$$
N_{\text {par }}=\max \left(\left\{\begin{array}{l}
N_{\text {par }, I}=\frac{I_{b, \max }}{I_{c, \text { max }}}=\frac{P_{b}}{n_{s} U_{c, \text { cutoff }}} \cdot \frac{R_{c}}{U_{c, o c}-U_{c, \text { cutoff }}} \\
N_{\text {par }, C}=\frac{E_{b}}{U_{s} C_{c}}
\end{array}\right)\right.
$$

From Ohm's law and assuming same current through cells in a module, the module internal resistance becomes the sum of the cell's resistances:

$$
R_{m}=\sum_{i=1}^{N_{s e r}} R_{c}=N_{s e r} R_{c}
$$

where the assumption of perfectly equal cells is used to simplify the equation. 
The total battery resistance is then computed from the assumption that modules connected in parallel share the same voltage difference at their extremes:

$$
R_{\text {bat }}=\sum_{i=1}^{N_{\text {par }}}\left(\frac{1}{R_{m}}\right)^{-1}=\left(\frac{N_{\text {par }}}{R_{m}}\right)^{-1}=\frac{N_{\text {ser }}}{N_{\text {par }}} R_{c}
$$

where equation 4.41 is used to fully define the battery resistance as a function of the cell resistance and configuration.

The assumption of linearly decreasing voltage as a function of the battery state of charge depends on the open-circuit potential, the cut-off to initial voltage ratio and the minimum SOC allowed to avoid damaging the battery pack:

$$
U_{b a t, o c}(S O C)=N_{s e r} U_{c, o c}\left(1-\frac{1-k_{c u t o f f}}{1-S O C_{l i m}} \cdot(1-S O C)\right)
$$

The battery state of charge can be computed from the previous step in the mission analysis as defined in equation 4.44. Here it is assumed that between two time-steps the electric requirements in term of battery voltage and current remain constant. This approximation improves as more time-steps are considered so it depends on the granularity chosen in the point mass analysis.

$$
S O C_{i+1}=S O C_{i}-\int_{t_{i}}^{t_{i+1}} \frac{1}{C_{b a t, i}} d t=S O C_{i}-\int_{t_{i}}^{t_{i+1}} \frac{U_{b a t, i} I_{b a t, i}}{E_{b a t, i}} d t
$$

With the battery fully defined at a given state of charge, the combination of battery voltage and current that satisfy the power requirement from the electric system are iteratively computed with the system of equations 4.45:

$$
\left\{\begin{array}{l}
I_{b a t}=\frac{P_{b a t, r e q}}{U_{b a t}} \\
U_{b a t}=U_{b a t, o c}(S O C)-I_{b a t} R_{b a t} \\
P_{b a t, \text { res }}=I_{b a t} U_{b a t}
\end{array}\right.
$$

The system is converged when the relative difference between the power requirement, $P_{b a t, r e q}$, and the power produced, $P_{\text {bat,res }}$, falls below the convergence threshold of $10^{-6}$ as suggested in [75].

The battery efficiency at a given step in the mission is function of the characteristics computed so far and of a technology factor, $\eta_{\text {bat,max }}$, that is used as the upper limit for the efficiency:

$$
\eta_{b a t}=\eta_{b a t, \text { max }}-\frac{I_{b a t}^{2} R_{b a t}}{U_{b a t, o c}(S O C) I_{b a t}}=\eta_{b a t, \text { max }}-\frac{I_{b a t} R_{b a t}}{U_{b a t, o c}(S O C)}
$$

Validation of the battery model is discussed in chapter 5.1.

Battery mass calculation differs from the other components of the hybrid electric powertrain as it depends both on the energy and power requirements. Given a specific energy, $\epsilon_{b a t}$, and power, $\hat{\rho}_{b a t}$, values at battery level, the mass is equal to:

$$
m_{\text {bat }}=\max \left(\frac{E_{\text {bat }}}{\epsilon_{\text {bat }}}, \frac{P_{\text {bat }}}{\hat{\rho}_{\text {bat }}}\right)
$$

Further exacerbating this constraint, the two values are inherently interdependent. As seen above, increasing the power requirement at the battery also increases the losses so more energy is extracted for the same result thus reducing the storage capability. As the losses are directly related to the internal resistance, a high-power battery would be designed with more conductive material and thinner electrolyte layers thus improving conductivity in the cells. However, the higher ratio between passive and active materials in the cells have the unwanted consequence of decreasing specific energy. This phenomenon is usually plotted on Ragone plots as the one in figure 4.12 where the battery specific energy is on one axis and its gravimetric power on the other.

Methods to model this phenomenon could not be found in literature so no correlation between the battery mass sizing parameters is provided. Nevertheless, since these values are main design variables set depending on technology expectations, this trade-off is kept in mind during the design space exploration and sensitivity studies. 


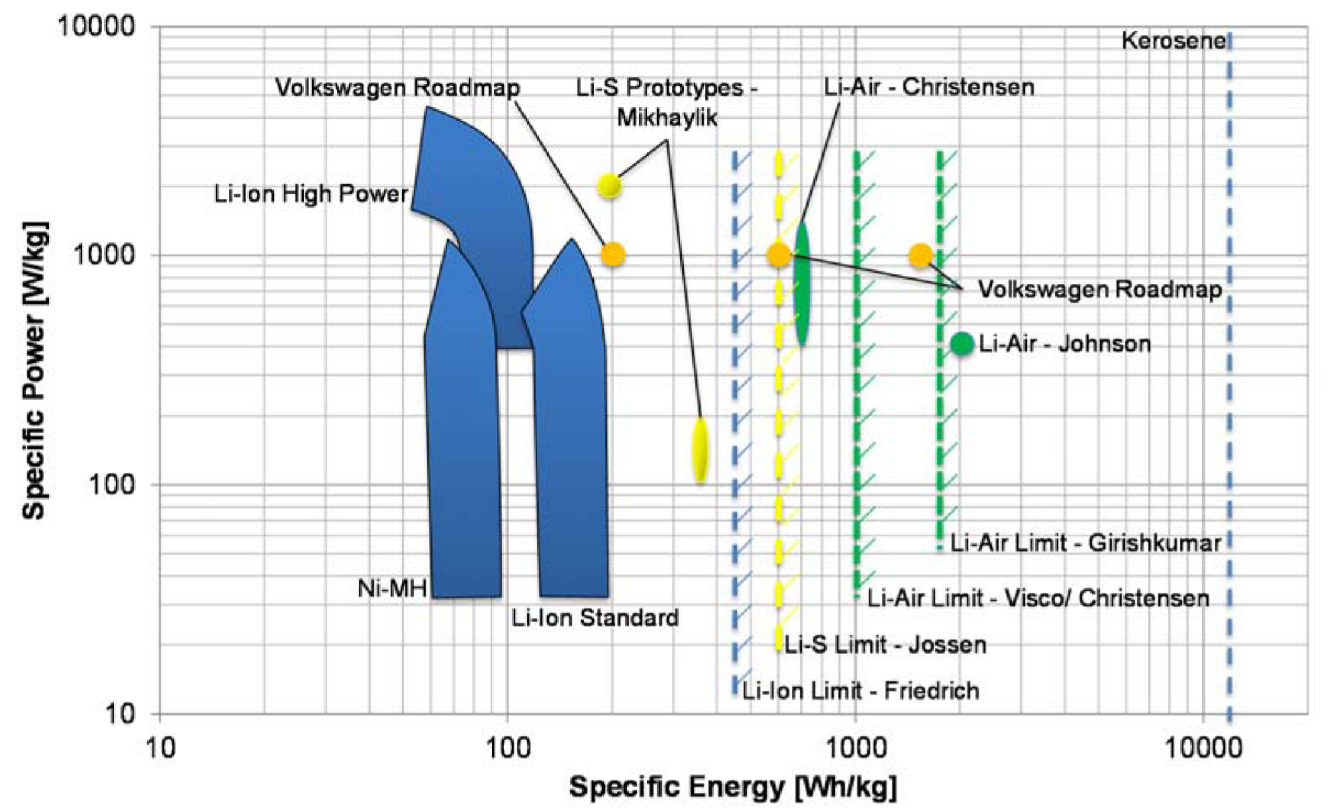

Figure 4.12: Ragone chart with battery characteristics predictions from reference [64].

\subsubsection{CRYO-COOLER}

As explained in section 2.2.1, using High Temperature Superconductors at cryogenics temperatures should allow for higher power densities, better specific power and efficiency by lowering all the aforementioned losses. HTS main target are the ohmic losses in the rotor and stator [76]. The impact of a cryo-cooler on an electric motor can be estimated at a preliminary level with the method proposed by Seitz et al. in reference [76]. Defining $P_{\text {input }}$, the required power input of the cooling device and $P_{\text {load }}$ its sizing power, the cooling performance coefficient relates them with the equation $C_{\text {cool }}=P_{\text {input }} / P_{\text {load }} . C_{\text {cool }}$ can also be defined in relation to the Carnot efficiency and mechanical efficiency of the cooling system:

$$
C_{\text {cool }}=\frac{C_{\text {Carnot }}}{\eta_{\text {mech }}} \quad \text { and } \quad C_{\text {Carnot }}=\frac{T_{\text {sink }}-T_{\text {load }}}{T_{\text {load }}}
$$

$T_{\text {sink }}$ is temperature of the external heat extraction unit and $T_{\text {load }}$ is the temperature of the system component that is being cooled. In the point mass analysis, the Carnot efficiency is kept constant at the value indicated by the designer. State of the art cryo-coolers reach an efficiency of $15 \%$ but a more optimistic value of $30 \%$ can be used for a YEIS of 2035.

Further defining $\vartheta_{e m}$ as the ratio between cryogenic heat losses and all the other motor losses, it is now possible to evaluate the HTS electric motor efficiency as a function of the EM efficiency without cooling, $\eta_{\text {em,bare }}$ and the parameters defined so far:

$$
\eta_{\text {em }}=\frac{P_{\text {em,out }}}{P_{\text {em,in }}}=\eta_{\text {em,bare }}-C_{\text {cool }} * \frac{\vartheta_{\text {em }}}{\vartheta_{\text {em }}+1} *\left(\eta_{\text {em,bare }}-1\right)
$$

Depending on the critical temperature and power load, the cryo-cooler impact on efficiency can thus be important and should not be neglected in the electric motor/generator sizing process.

\subsection{CONSTRAintS}

An essential part of commonly used conceptual design procedures is the constraint analysis [57, 66] that allows to translate the customer and legislation requirements into values for the wing and power loading (or thrust loading) such that the final design satisfies the constraints. As the internal components of a HEP are 
sized through their power requirements, a power-loading constraint diagram is preferred to a thrust to weight ratio one regardless of the propulsive device used.

The locations at which the power constraints are satisfied are represented by curves on a diagram with the power loading on one axis and the wing loading on the other. On a power loading diagram, the space above a curve represents the $[\mathrm{W} / \mathrm{P}, \mathrm{W} / \mathrm{S}]$ combinations that do not satisfy the constraint as the propulsive system is under-powered while the area below is the feasible design space bounded on the right by the maximum wing-loading (i.e. the smallest wing admissible to satisfy the lift constraints).

The power constraint is obtained from statistical data or by solving the equilibrium of forces along the longitudinal axis of the aircraft. These constraints are function of the wing loading, aircraft state (i.e. altitude, speed, climb/descent gradient, weight fraction), aerodynamic characteristics and other aircraft performance parameters. The wing loading constraints are obtained by solving the equilibrium system along the vertical axis. These are generally function of the maximum lift coefficient and flight state. However, when the propulsion system is used to augment the lift capabilities of the aircraft, the wing loading is affected also by the amount of propulsive power.

The result obtained for the propulsive power are valid for any HEP internal architecture as the underlying assumption of equilibrium of forces is not affected by the type and relative size of the propulsion system components. However, the propulsive power loading cannot be directly used to size the powertrain, but several corrections must be applied to translate it into shaft power loading at take-off conditions. The corrections considered in this study are:

- Aero-propulsion system interactions

- Propulsive efficiency

- Atmospheric and flight speed conditions

- One Engine Inoperative (OEI) conditions

These values are affected in different ways by the number, type and relative size of the propulsive system elements. Thus, depending on architecture type and hybridization factor, it is possible that more beneficial power and wing loading (i.e. less powerful propulsors and smaller wing) can be used as starting point in the design process. Each correction is discussed in more detail in the following sections.

\subsubsection{AERO-PROPULSIVE CORRECTION}

Modern day aircraft tend to be designed with only two engines as turbo-machinery is expensive to acquire and maintain. Installing more engines is usually necessary only when very powerful or large aircraft are designed as the improvement to engine reliability allows the use of only two propulsors to satisfy even the more stringent certifications (e.g. ETOPS) [77]. Moreover, an excessive downsize of turbo-machinery negatively impacts its overall efficiency and specific weight.

On the other hand, electric machines suffer little to no penalty due to scalability, so they can be sized to the required power while maintaining very high efficiencies. For this reason, the use of electric motors opens the way to novel aircraft configurations that would leverage beneficial aero-propulsive interactions. Example of this are the use of several small propellers spread along the wing span that could improve the wing lift capabilities while reducing its weight thanks to a more favourable weight distribution [58, 59]. Using wing-tip mounted propellers could also be a way to reduce the strength of vortexes generated when lift is produced thus reducing the overall drag [78]. Finally, electric motors are highly controllable in terms of output power and can be switched on and off in short time and without the need of additional sub-systems. Thus, they could be used as means of aircraft control through the production of differential thrust, possibly allowing for the downsizing of the control surfaces [79].

Implementing accurate predictions for the aero-propulsion interaction is outside the scope of the current study, however their benefits are deemed essential in the study of novel propulsion system architectures made feasible by the introduction of electric machines. Therefore, the method allows the correction of the lift and drag polar using segment specific parameters such as $C_{L}$ increments in take-off, climb, cruise and landing and multiplicative factors for each drag coefficient component. These polars are used both in the constraints 
analysis and in the point mass estimations thus ensuring that the designer assumptions are coherently considered throughout the sizing process.

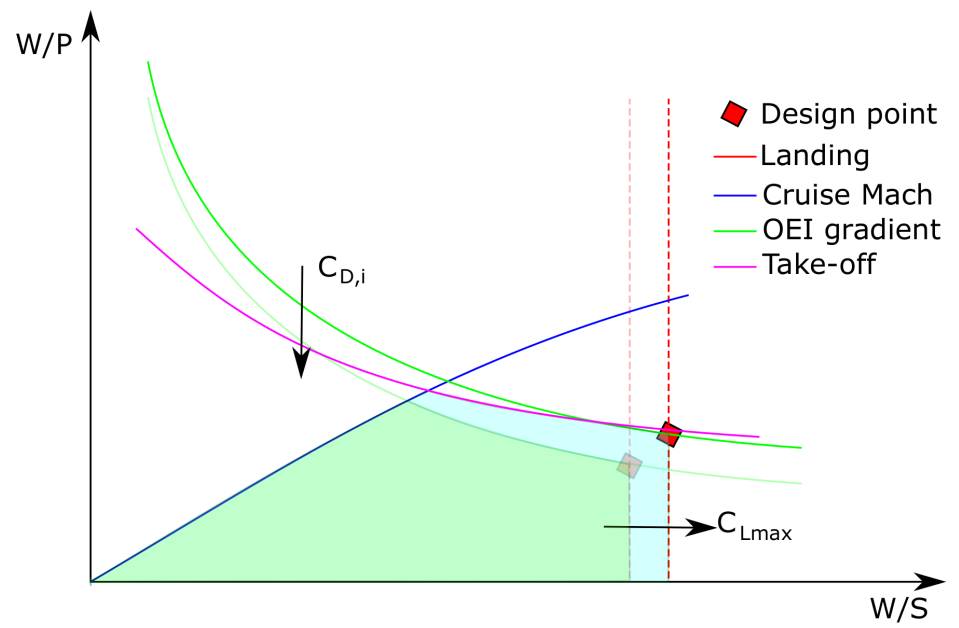

Figure 4.13: Changes in the power loading constraint diagram due to aero-propulsive interactions.

Figure 4.13 shows a sample constraint diagram where assumption for higher $C_{L, \text { max }}$ and lower induced drag have been made. The net result is an increase of the feasible design area with an improvement to both the shaft power and wing loadings.

\subsubsection{PROPULSION EFFiCIENCY CORRECTION}

The results obtained from the equilibrium of forces along the aircraft longitudinal axis cannot be directly used for sizing purposes as they do not consider the losses due to the propulsive device. In this study, the transformation of shaft power into usable thrust is accomplished by a propeller and the characteristics of the propulsive device losses are described by the propeller efficiency defined as the ratio between propulsive power and shaft power:

$$
\eta_{P}=\frac{P_{P}}{P_{s h}}=\frac{T \cdot V}{P_{s h}}
$$

A variable propeller efficiency estimation based on the disk actuator theory has been implemented to capture the influence of the flight state on this ratio as explained in section 4.2.1. As for the aero-propulsion interaction correction, it is possible that the choice of propulsive system external architecture influences the propulsive efficiency and consequently the power loading results. However, the propulsive efficiency of modern turboprops is already very high when the flight Mach number is kept below 0.6-0.7 so little to none improvements are expected with the electrification of the powertrain. Larger benefits could be instead obtained for turbofan designs due to the possibility of increasing the bypass ratio or the de-coupling of the fan from the turbine stages [72].

As the propulsive efficiency is computed for each PPU depending on flight state and propeller geometry, the impact of design parameters such as the propeller disk loading or number of blades can still be studied on the constraint diagram as shown in figure 4.14. Improvements to overall propulsive efficiency lead to a higher power loading requirement thus allowing for smaller machines to be installed while still satisfying the constraints.

\subsubsection{POWER-LAPSE CORRECTION}

Air breathing engines performances are affected by the atmospheric conditions. The impact that the flight altitude has on the performance of a power machine is captured in the power lapse coefficient defined as the ratio between shaft power produced at a given flight condition over the corrected shaft power at sea-level static (SLS) conditions. This coefficient depends on the machine characteristics, the flight altitude and flight Mach number. If these relations are known, they can be supplied to the tool in the form of a table taking as 


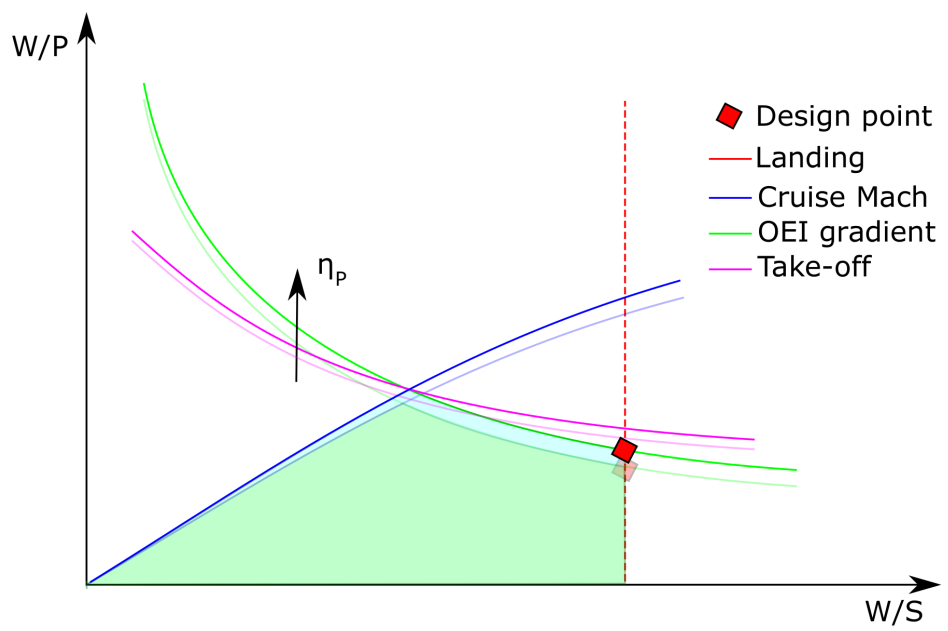

Figure 4.14: Changes in the power loading constraint diagram due to changes to the propulsive efficiency.

inputs the altitude, flight Mach and control setting and having the power lapse as output. However, when the engine map is not provided, an analytical estimation of the power lapse as explained in reference [80] is used in its place.

For gas-turbine engines in subsonic conditions, the power lapse factor depends mainly on the flight altitude. As the altitude increases, the air density decreases thus diminishing the mass flow rate and limiting the power that can be extracted from the turbine. On the other hand, the increase in altitude is related to lower air temperatures that improve the specific thrust/power of the engine. The degradation of mass flow and improvements of specific power are combined to obtain the variation of the power with altitude as defined in equation 4.51:

$$
\Upsilon=\frac{P_{s h, g t}}{P_{s h, g t, 0}}=\left(\frac{\rho}{\rho_{0}}\right)^{k}
$$

Where $\rho$ is the air density at altitude, $\rho_{0}$ is the ISA air density at sea-level and $\mathrm{k}$ is a coefficient that has a value of roughly 0.75 for the troposphere.

Electric machines such as electric motors and generators are not affected by the changes in altitude. Their power lapse can be assumed equal to one indicating that they are not appreciatively influenced by changes in altitude or flight Mach. Therefore, the propulsive power loading can be converted to shaft power loading using equation 4.52:

$$
W / P_{s h, 0}=\frac{W / P_{s h, g t}}{\Upsilon}+W / P_{s h, e m}=W / P_{s h} \cdot\left(\frac{1-D o H_{P, s e g}}{\Upsilon}+D o H_{P, s e g}\right)
$$

Where the variable $\mathrm{DoH}_{P, \text { seg }}$ is introduced to describe the ratio between electric and total shaft power at altitude as defined in equation 4.53:

$$
\mathrm{DoH}_{P, \mathrm{seg}}=\frac{P_{s h, e m}}{P_{s h}}=\sum_{i=1}^{N_{P P U}}\left(\frac{P_{s h, i, e m}}{P_{s h, i}} \cdot \frac{P_{s h, i}}{P_{s h}}\right)
$$

Differently from the degree of hybridization defined in chapter 3.1, this parameter changes during the mission and it will be different for each constraint considered depending on the intended operation of the hybrid electric powertrain. Since the conventional engine power lapse is less than 1 for altitudes above sea level, in figure 4.15 it can be observed how a higher electrification of the powertrain allows for a relaxation of the power loading requirements that need to be satisfied at high altitudes. Little to no impact will be observed for constraints at sea-level. The increase of power loading is usually regarded as a beneficial change as it leads to a lighter and less expensive propulsion system. However, these improvements are counteracted by the increase of weight mainly due to the mass of the battery and electric system. 


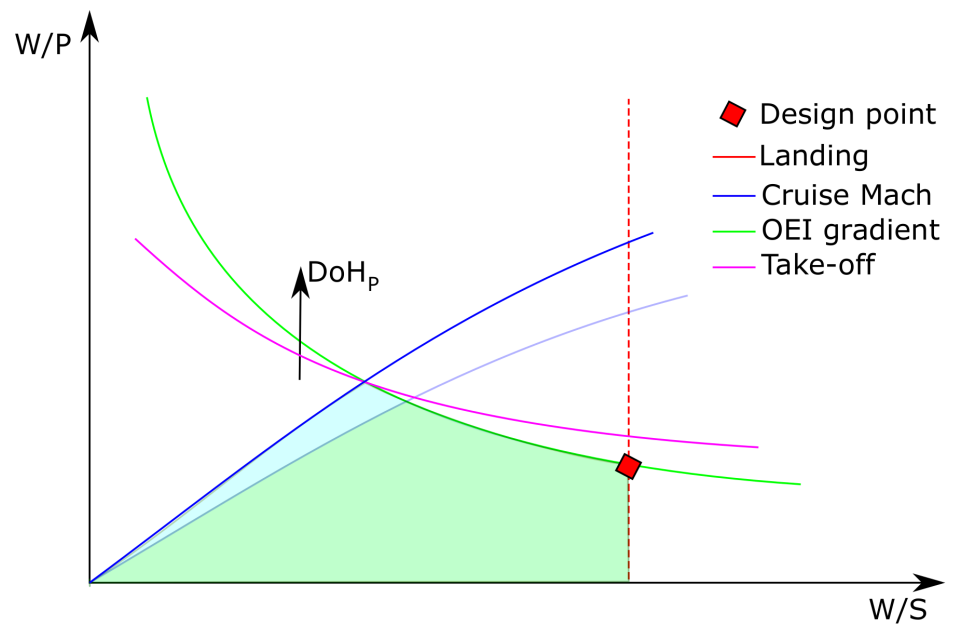

Figure 4.15: Changes in the power loading constraint diagram due to the segment degree of electric hybridization.

\subsubsection{OnE ENGINE INOPERATIVE CORRECTION}

Aviation agencies indicate that to certify an aircraft it needs to be able to satisfy certain regulations even in the event of a propulsive device failure. On the power constraint diagram this can translate to a restriction of the design space for twin engine designs as each powerplant needs to be twice as large as initially predicted from the force balance analysis for the considered constraint. For increasing number of powerplants the loss of a PPU is less critical, however this is compensated by a more stringent requirement in the case of the climb gradient regulations.

In non-hybrid multi-engine aircraft the powerplants are all the same type and power rating to keep the acquisition and maintenance cost contained. This then means that all the propulsive units can produce the same amount of thrust. The most critical condition is realized when the powerplant furthers from the aircraft centre of gravity fails as this will generate the highest yaw moment for a set loss of thrust that then needs to be trimmed with the control surfaces. The power loading equation needs to be re-computed accounting for the additional trim drag and eventual drag due to wind milling/stopped propeller. Then, the OEI power loading correction factor is simply:

$$
k_{O E I}=\frac{N_{P P U}-1}{N_{P P U}}
$$

When more of one type of powerplants is installed, it is possible that the power ratings will be different, and this needs to be considered in the OEI constraint computation. When distributing the thrust in a heterogeneous way the correction factor becomes function of the largest propulsive power unit:

$$
k_{O E I}=\frac{P_{P}-\max \left(P_{P, i}\right)}{P_{P}}=1-\max \left(\Theta_{i}\right)
$$

Where $\Theta_{i}$ is the average value attained by the propulsive power share for the considered constraint. If the propulsive system makes use of parallel power units (i.e. the propeller shaft is driven simultaneously by a conventional turbo-shaft engine and an electric motor), not all shaft power at the propeller would be lost in case of an engine failure. Equation 4.55 is applicable if a total PPU failure is expected, however, in case that only the gas-turbine stops working, the OEI correction coefficient becomes:

$$
k_{O E I}=\frac{P_{P}-\max \left(P_{P, g t, i}\right)}{P_{P}}
$$

The partial PPU failure lessens the severity of the OEI constraint so it should be chosen under the assumption that the regulations would permit the fulfilment of the requirements in this way. This will not be the case for this study as the more conservative assumption is used when designing a parallel HEA.

Figure 4.16 shows how an increase of the number of PPU can affect the design space in terms of constraint requirements. Clearly, only OEI constraints are affected by this correction, so it is possible that improvements to the power loading are limited as an All Engine Operative (AEO) requirement could be the sizing one. 


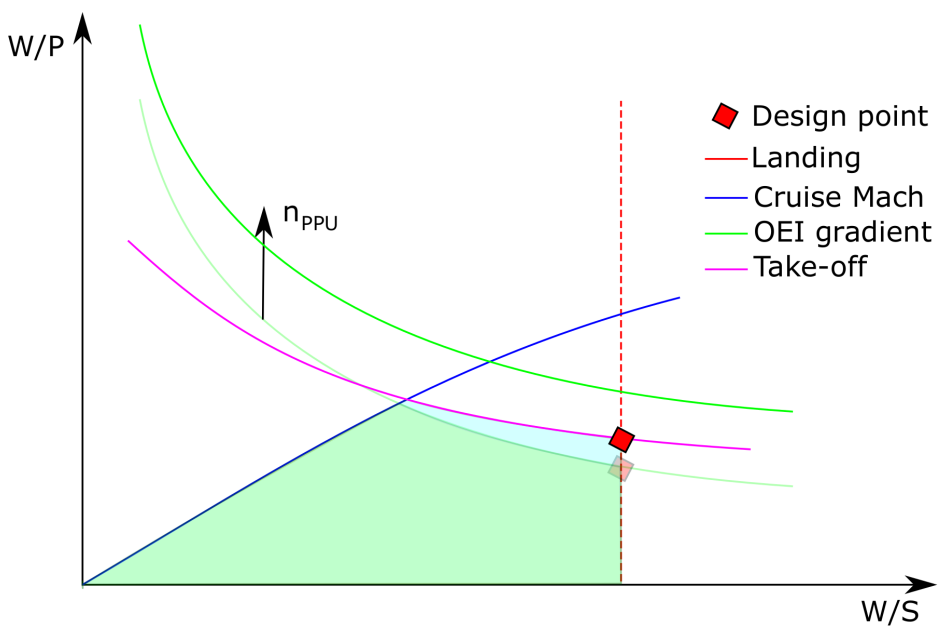

Figure 4.16: Changes in the power loading constraint diagram due to the number of propulsive devices.

\subsection{Point PERFormance ANALYSis: HEP SySTEM ANALYSis}

The proposed method uses the mass point analysis module to first compute the propulsive power necessary to fly the mission and then propagate it "upstream" along the power-paths constituting the HEP system. Through efficiency corrections at each component, the power at the energy sources can be evaluated for each step in the mission. By time-integration of these values, it is then possible to compute the energy consumption for the sizing mission. The values of power attained along the power-paths depend both on the HEP control parameters and on each components power conversion efficiency as explained in the following sections. The point performance analysis is not only used to compute the energy consumption, but the maximum values of the power obtained at a given point in the mission, corrected to sea-level static when necessary, are used as sizing parameters in the mass and geometry estimations.

\subsubsection{Propulsive power eVAluation}

With the conclusion of the constraint analysis, estimations for the power and wing loading are obtained. Using the MTOM computed in previous process iterations, the total shaft power can be used in the point mass analysis to evaluate the energy consumption and propulsive system power requirements. The first step for each estimation as part of the point mass analysis is the computation of the propulsive power requirement. In this regard, the mission segments are subdivided in two groups.

The first group comprehend all the segments in which the propulsive power can be directly computed from the flight condition and aerodynamic characteristics of the aircraft. These segments are the taxi, cruise, descent and landing phases and they are characterized either by a uniform motion (taxi, cruise, landing) or by an acceleration that can be derived directly from the flight condition. The second group comprehend the take-off and climb phases and it is characterized by the need of an additional equation to compute the aircraft acceleration as a function of the total shaft power derived from the constraint analysis.

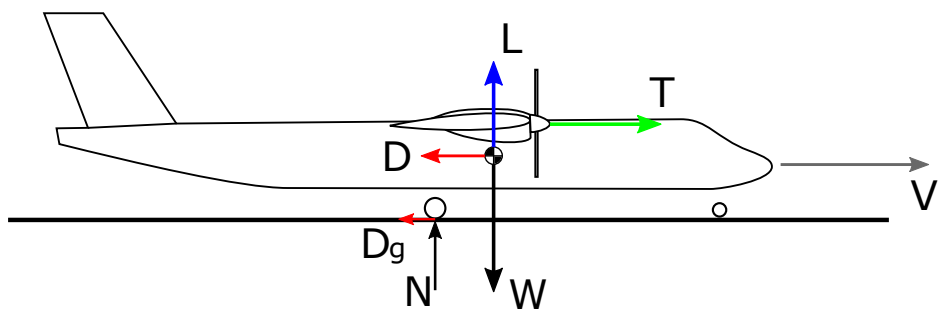

Figure 4.17: Free-body diagram of aircraft on ground.

Figure 4.17 shows the free-body diagram for an aircraft moving on the ground. The system of equations of 
motion that define this condition is:

$$
\left\{\begin{array}{l}
F=m \cdot \frac{d V}{d t}=T-D-D_{g} \\
D=\frac{1}{2} \rho V^{2} S C_{D} \\
C_{D}=C_{D, 0}+k \cdot C_{L}^{2}+\Delta C_{D}, D_{g}=\mu N=\mu(W-L)=\mu\left(m \cdot g-\frac{1}{2} \rho V^{2} S C_{L}\right)
\end{array}\right.
$$

In these equations, the acceleration of the aircraft is function of the thrust, $\mathrm{T}$, the aerodynamic drag, $\mathrm{D}$ and the ground friction, $D_{g}$. The air-density, $\rho$, is function of the altitude and air temperature, the reference area, $\mathrm{S}$, is fixed during the point mass analysis and the runway friction coefficient, $\mu$, is provided by the user depending on the surface type. The aircraft mass, $\mathrm{m}$, and speed, $\mathrm{V}$, depend on the previous condition and on the changes due to fuel consumption and acceleration respectively. The aerodynamic coefficients $C_{L}$ and $C_{D}$ depends on the aircraft geometry, orientation with which it moves through air and Mach number:

$$
\begin{gathered}
C_{L}=C_{L, 0}+C_{L_{\alpha}} \cdot \alpha+\Delta C_{L, \text { flap }} \\
C_{D}=C_{D, 0}+k \cdot C_{L}^{2}+\Delta C_{D, \text { flap }}+\Delta C_{D, \text { undercarriage }}+\Delta C_{D, \text { wave }}
\end{gathered}
$$

The system is under-defined and another condition is required for its solution. For the taxi phase, the taxiing speed is assumed constant. In this way the thrust becomes equal to the sum of the aerodynamic and friction drag forces and the system is solved. For the take-off phase, a constant throttle percentage is assumed so that the thrust becomes a function of the shaft power and flight state as the propeller efficiency changes with speed and air density.

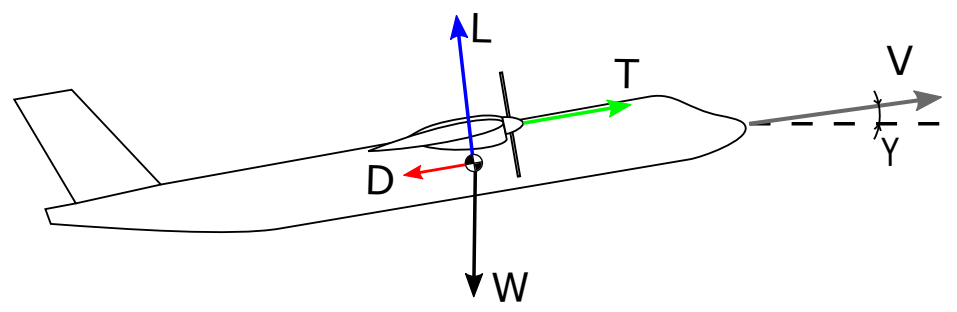

Figure 4.18: Free-body diagram of aircraft in flight.

Figure 4.18 shows the free-body diagram for a flying aircraft. In this case the system of equations become:

$$
\left\{\begin{array}{l}
F=m \cdot a=T-D-m \cdot g \cdot \cos (\gamma) \\
D=\frac{1}{2} \rho V^{2} S C_{D} \\
L=\frac{1}{2} \rho V^{2} S C_{L}=m \cdot g \cdot \sin (\gamma)
\end{array}\right.
$$

Two extra conditions are required to solve the system:

- Take-off: for the airborne part of the segment, the two extra conditions are the throttle percentage and the climb-out flight angle, $\gamma$.

- Climb: similarly to the take-off, for the climb phase the throttle percentage is specified to correlate the thrust to the propulsive system shaft power and flight condition. Instead of defining the flight path angle, the acceleration is specified as a function of the flight state by assuming a constant Calibrated Air Speed (CAS) up to the cruise Mach number and then a constant Mach number until the top of the climb segment is reached. Maximum CAS limitations also apply under FL100 so if such speed is achieved below this flight level, the velocity is kept constant leading to a larger share of propulsive power usable for climbing.

- Cruise: for the cruise condition it is assumed that the motion is uniform (i.e. $d V / d t=0$ ) and that the aircraft remains at constant altitude (i.e. $\gamma=0$ ).

- Descent: in descent, the acceleration is function of the flight state using the same approach as for the climb condition. However, a constant rate of descent $(R O D=V \cdot \sin (\gamma))$ is assumed instead of a constant shaft power percentage. 
- Landing: for the airborne phase of the landing segment, a constant approach speed and approach angle are assumed.

With this, the propulsive power at each step of the mission can be computed as the product of flight speed and thrust:

$$
P_{P}=T \cdot V
$$

And then propagated to the rest of the propulsive system as explained in the following section.

\subsubsection{POWER-PATHS COMPUTATIONS}

The shaft power produced by the electric motor and turbo-shaft engine are combined in a parallel PPU and supplied to the shaft of the propeller. Since the required propulsive power and propeller efficiency are already known at this stage, it is possible to compute the gearbox output shaft power as a function of the propulsive power ratio, $\Theta$, as defined in equation 4.62 :

$$
P_{g b}=\Theta_{i} \frac{P_{P}}{\eta_{P}}
$$

Then, depending on the shaft power ratio, the shaft power required from the electric and conventional machines can be evaluated by considering any mechanical transmission loss using the gearbox efficiency:

$$
\begin{gathered}
P_{e m}=\varphi \frac{P_{g b}}{\eta_{g b}} \\
P_{g t}=\Upsilon(1-\varphi) \frac{P_{g b}}{\eta_{g b}}
\end{gathered}
$$

$P_{g t}$ is the shaft power that a gas-turbine engine needs to produce during flight. However, as seen previously, the performances of air-breathing engines degrade with altitude so the sizing power for this machine needs first to be corrected by the power lapse, $\Upsilon$, as defined in equation 4.51 . Then the maximum of these resulting values along the entire mission can be used as the reference value for weight and size estimations of the engine.

The output shaft power of the engine is used to compute the fuel mass flow using its specific device map that takes as other inputs the flight Mach number, the altitude and temperature difference from the ISA standard. To evaluate the overall system efficiency, the fuel flow can be expressed in terms of fuel power through its specific heating value (i.e. specific energy) $\epsilon_{f u e l}$. The efficiency of the conventional machine is then expressed as the ratio between gas-turbine shaft power and fuel source power:

$$
P_{f u e l}=\epsilon_{f u e l} m_{\dot{f u e l}}=\frac{P_{g t}}{\eta_{g t}}
$$

The power converted by the electric motor to turn the propeller is carried through cables and sourced by the PMAD:

$$
P_{P M A D}=\frac{P_{e m}}{\eta_{e m} \eta_{c b}}
$$

If part of the power provided to the PMAD is produced by an electric generator - turbo-shaft system, the shaft power of the conventional machine is corrected by the losses of the generator, the cables and PMAD:

$$
P_{e g t}=\Upsilon(1-\phi) \frac{P_{P M A D}}{\eta_{e g} \eta_{c b} \eta_{P M A D}}=\Upsilon \frac{P_{e g}}{\eta_{e g}}
$$

Where the sizing power of the electric generator, $P_{e g}$, is function of the electric power ratio and the efficiencies of the PMAD and cables connecting them together. As the turbo-shaft is an air-breathing machine, its shaft power used for sizing purposes is first corrected by the power lapse coefficient. With the gas-turbine power known, its fuel flow and fuel power can be computed as previously presented and added together with all fuel-using PPUs to evaluate the overall consumption.

The rest of the power provided to the PMAD comes from the battery packs. The power that needs to be generated by the batteries, $P_{b a t}$ depends on the PMAD efficiency, the electric power ratio and the cable efficiency. 
Depending on the relative position of battery and PMAD, the cable efficiency can change as its losses are a function of its length. By also using the battery efficiency, variable with power and voltage requirements, the battery power equivalent of fuel power (i.e. at the energy source) can be computed:

$$
P_{\text {bat }}=\phi \frac{P_{P M A D}}{\eta_{\text {bat }} \eta_{c b} \eta_{P M A D}}=\frac{P_{\text {bat }, \text { out }}}{\eta_{\text {bat }}}
$$

As for the electric generator, the total battery nominal power is the sum of all the power requirements at a point in time. If more than one electric generators and battery packs are in the design, the user indicates how much of the total power or energy requirement is taken upon by the single object. This coefficient can also be used to ensure the required redundancy of the critical power producers for safety concerns as the single component can be over-sized in terms of power and energy (only the battery).

\subsubsection{ENERGY CONSUMPTION ESTIMATION}

Total energy results from the produced power at the energy sources and time interval for which this power is required. Given $N$ time-steps during the total mission, the fuel and battery energy are computed using the Riemann trapezoidal sum:

$$
\begin{gathered}
E_{f u e l}=\sum_{i=1}^{N}\left[\left(\frac{\dot{m}_{f u e l, i}+\dot{m}_{f u e l, i-1}}{2}\right) \cdot \epsilon_{f u e l} \cdot\left(t_{i}-t_{i-1}\right)\right] \\
E_{\text {bat }}=\sum_{i=1}^{N}\left[\left(\frac{P_{b a t, i}+P_{b a t, i-1}}{2}\right) \cdot\left(t_{i}-t_{i-1}\right)\right]
\end{gathered}
$$

Electric batteries have a minimum capacity under which they become irreparably damaged, so they should never be fully discharged. This critical energy capacity is usually defined through the minimum State Of Charge (SOC) defined as the ratio between minimum and fully charged capacity. This value changes depending on the chemistry of the selected battery and it is supplied as a design variable along the electro-chemical cell specific energy and power. With this parameter known, the total energy required for the sizing mission is over-sized accordingly.

With this, the point mass analysis of the mission is concluded and the sizing powers and energy requirements are translated into masses and dimensions of the propulsive system components by the aircraft sizing module. If the outputs have not yet converged, another iteration with the new estimates for the relative shaft power values and updated weights is performed.

\subsection{HyBRID ELECTRIC ARCHITECTURE KEY PERFORMANCE INDICATORS}

When it comes to aircraft design, novel technologies and propulsion system architectures, there is the need to define a select group of parameters to understand if the use of hybrid electric propulsion can produce benefits. These are identified with the acronym KPIs (Key Performance Indicators). The KPI identified for this study and discussed in the sections below are the design masses, energy consumption and system efficiency, pollutant emissions and direct operating cost.

\subsubsection{HYBRIDIZATION AND EFFICIENCY}

Commonly used KPI when comparing aircraft are the design masses such as their maximum take-off mass, the operative empty mass and the ones related to their performances and mission, the payload and fuel mass. The latter one is of high interest as its reduction is one of the main objectives that drive the research into the electrification of the propulsive system. The fuel mass reduction is supported both by airline operators and regulatory agencies as it directly correlates with lower operating costs and fuel related emissions.

The problem with using the fuel mass as the only objective in a HEP is that the global minimum can be achieved by simply removing all fuel consuming machines and designing a universally electric aircraft. However, due to the disadvantageous exchange of electric and fuel energy in terms of mass, the final design would weight much more possibly leading to an increased total mission energy requirement. As there are costs 
related to the production of electric energy, both in terms of money and emissions, the universally electric aircraft could then be more expensive to operate and more polluting. Therefore, it makes sense to include the total energy among the KPIs when comparing hybrid propulsion systems.

The amount of energy required to fly a certain mission is also influenced by how efficiently the source power is converted to usable thrust. With the power requirements at the propulsive devices and energy sources known for each timestep along the mission, it is possible to derive the overall propulsive system efficiency defined as the ratio between propulsive power and supplied power [72]:

$$
\eta_{\text {ov }}=\frac{P_{P}}{P_{\text {bat }}+P_{\text {fuel }}}=\frac{P_{P}}{P_{\text {Supply }}}
$$

Although the electric power-path has more energy conversions when compared with a fuel based sub-system, the efficiency of each electric component is usually very high (around 90\%). For this reason, it is expected that an increase of electrification of the propulsive system could reduce the overall losses between source and propulsive device. If the mass penalty of this increased hybridization is not excessive, the higher efficiency translates into lower total energy consumption and consequently lower overall emissions.

Another way to assess the efficiency of the design is to compare the distance that it can cover for unit of energy consumed. In a fuel burning aircraft this can be done through the Specific Air Range (SAR) defined as the distance flown for mass of fuel consumed. Clearly, this parameter cannot be used in HEP estimations as it would grow to infinity as the electrification share is increased. On the other hand, the parameter can be re-defined as a function of total energy as done by the authors of reference [76]. The Energy Specific Air Range (ESAR) function as a generalization of the SAR for a generic energy source and it is defined as the change in range due to a change in total energy:

$$
E S A R=\frac{d R}{d E}=\frac{V \cdot L / D}{T S P C \cdot W}=\frac{\eta_{o v} \cdot L / D}{W}
$$

where the Thrust Specific Power Consumption (TSPC) is the ratio between sources' power and generated thrust. By dividing the TSPC by the flight velocity $V$, the system overall efficiency is introduced in this figure of merit. This parameter can be used as an objective function if the goal of the optimization is to fly a mission with the least amount of energy. Differently from other figures of merit discussed above (block fuel and energy), the ESAR is directly influenced by the aircraft weight so it could prove a better choice in the investigations of hybrid electric propulsion as the mass penalty of the aircraft electrification is better represented.

\subsubsection{EMISSIONS}

One of the main reasons why the electrification of the propulsive system is of high interest in recent years is that electric energy has zero in-flight emissions. This is seen as a possible way to achieve the targets sets by the international aviation regulatory agencies in terms of pollutions and greenhouse gases reduction. Due to this importance, the emissions of the design are incorporated among the studied KPIs.

As a full engine model is not implemented in the selected software and its complexity would exceed the requirements of a conceptual design tool, the mass of pollutants released during fuel combustion can be defined through their Emission Index. The EI is defined as the ratio between the mass of pollutant in grams over the mass of fuel consumed in kilograms [81]:

$$
\text { EI } I_{\text {substance }}=\frac{m_{\text {substance }}}{m_{\text {fuel }}}
$$

Table 4.2 reports the emission index for the substances that are released by air-breathing engines. Carbon dioxide $\left(\mathrm{CO}_{2}\right)$ and water vapour $\left(\mathrm{H}_{2} \mathrm{O}\right)$ are products of the complete (i.e. ideal) combustion and their EI can be computed from the stoichiometric analysis of the fuel oxidation process. Therefore these EIs are only function of the fuel used. On the other hand, the amount of carbon monoxide (CO), un-burnt hydrocarbons (UHC), soot and nitrogen oxides $\left(N O_{x}\right)$ depend on the thrust setting, engine type, flight altitude and airspeed [82]. Of these pollutants, $\mathrm{CO}_{2}$ and $\mathrm{NO}_{x}$ have the largest impact on global warming. The latter has complex 


\begin{tabular}{ccc}
\hline Substance & Emission Index [g/kg fuel] & Emission depends on \\
\hline $\mathrm{CO}_{2}$ & 3150 & fuel consumption \\
$\mathrm{H}_{2} \mathrm{O}$ & 1250 & \\
\hline $\mathrm{CO}$ & $2.3-36.4$ & thrust setting: maximum \\
$\mathrm{UHC}$ & $0.1-4.4$ & produced at idle \\
\hline Soot & $0.2-2.6$ & thrust setting: maximum \\
$\mathrm{NO}_{x}$ & $4.3-20.8$ & produced at full thrust \\
\hline
\end{tabular}

Table 4.2: Indicative emission indices for modern gas-turbine engine adapted from [82, 83]

interactions with the atmosphere depending on the altitude as it contributes to the reduction/increase of the ozone concentration at stratospheric and tropospheric altitude respectively.

To more easily consider the impact of different designs and operations strategies on the environment, a Global Warming Potential model can be used. The $\mathrm{CO}_{2}$ equivalent GWP of a substance is defined as the global warming due to a kilogram of the pollutants with respect to the global warming through emission of a kilogram of $\mathrm{CO}_{2}$ [84]:

$$
G W P_{\text {eq, substance }}=\frac{G W P_{\text {substance }}}{G W P_{\mathrm{CO}_{2}}}
$$

The GWP depends on the photo/chemistry interactions of the substance, the altitude at which it is emitted and by the time horizon chosen for the comparison [85]. The time horizon needs to be chosen as different substances have different lifetime in the atmosphere with common lengths between 20 and 100 years.

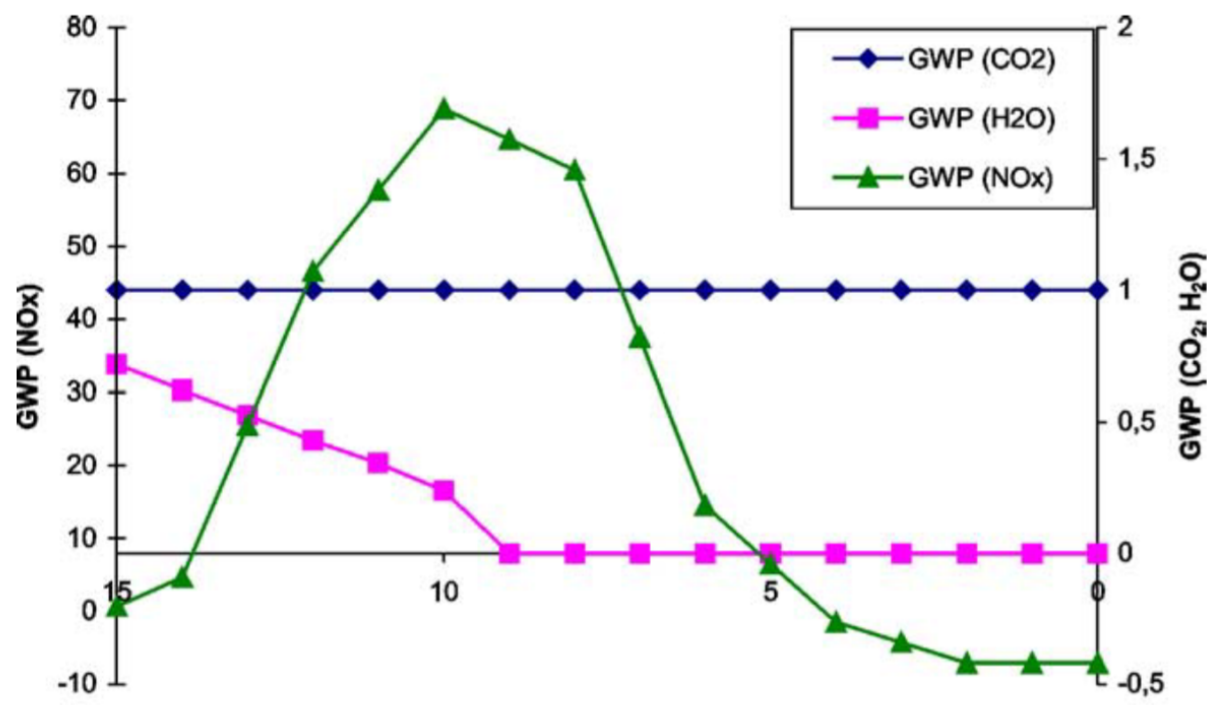

Altitude [km]

Figure 4.19: Global Warming Potentials of $\mathrm{CO}_{2}, \mathrm{H}_{2} \mathrm{O}$ and $\mathrm{NO}_{x}$ as reported in [86]

The evaluation of the substance GWP requires complex 3D climate models but, as modelling these exceeds the scope of this study, the parametric method presented in [86] is used to derive the GWPs. In this study, the impact of the main pollutants with a time horizon of 100 years is tabulated as a function of altitude so it can be directly used in the evaluation of the total GWP obtained as the sum of the substances warming potential integrated along the sizing mission.

Figure 4.19 shows the change in GWP with altitude as reported in [86]. It can be observed that at altitude of interest for turbo-prop aircraft (between sea-level and $8 \mathrm{~km}$ ), the water vapour has negligible global warming impact. The $N O_{x}$ emissions have a negative GWP below $4 \mathrm{~km}$ (i.e. they have a cooling effect) as at these altitudes they react with methane $\left(\mathrm{CH}_{4}\right)$, a gas with very strong greenhouse effects. However, the GWP of the nitrogen oxides grows rapidly reaching a peak of 70 times the strength of $\mathrm{CO}_{2}$ at the limits of the troposphere 
where the $N O_{x}$ reacts with the ozone layer, reducing the shielding effect against ultraviolet radiation from the sun.

With the inclusion of the GWP correction, an estimation of the tank-to-propeller $\mathrm{CO}_{2}$ emissions is done. This figure of merit is the one usually considered in HEP studies as it is the one used also by airlines and aircraft manufacturers. However, similarly to the fuel case, optimizing a design with respect to this performance indicator pushes the design to a universally electric aircraft as the electric energy with which the fuel is substituted does not have any costs in terms of in-flight emissions. As discussed in [87], it is interesting to also observe the well-to-propeller emissions as this incorporates the $\mathrm{CO}_{2}$ emissions related to the production of electric energy, thus making this result sensitive to the increase of total energy related to the higher mass associated with battery-based systems.

To account for the inefficiency in the fuel supply chain, $0.61 \mathrm{~kg} \mathrm{CO}_{2} / \mathrm{kg}$ of fuel [88], equivalent to $0.05 \mathrm{~kg}$ $\mathrm{CO}_{2} / \mathrm{kWh}$ of fuel energy assuming $11.9 \mathrm{kWh} / \mathrm{kg}$ for kerosene [41], are added to the emissions estimations. For the electric energy part, the production and distribution have an average of $0.42 \mathrm{~kg} \mathrm{CO}_{2} / \mathrm{kWh}$ electricity [89]. This value depends on the way the electric energy is produced; it tends to zero as renewable energies make up a larger share of the electricity production share. 


\section{5}

\section{VALIDATION}

In this chapter the models presented in chapter 4 are compared to experimental data to check their accuracy and precision in predicting the components characteristics and aircraft KPIs. Firstly, the propulsion system components are compared with data found in literature. The models are used in isolation from the rest of the sizing model to validate their prediction. Then, a conventional turbo-prop design is compared to the chosen reference aircraft to validate the overall model, the mass computation and the point performance estimations.

\subsection{COMPONENTS VALIDATION}

The validity of the methods to estimate the performances of the electric components of the HEP is discussed in this section. The PMAD sub-system elements are not present in this validation work as a constant efficiency is assumed throughout their working range.

\subsubsection{PROPELLER}

The chosen reference aircraft, the ATR72, uses two modern Hamilton-Sunstrand F568 propellers to convert the shaft power into usable thrust. The F568, visible in figure 5.1a, is a six-bladed propeller with a diameter of $3.93 \mathrm{~m}$ and highly swept blades [90].

The propeller nominal rotational speed is $1200 \mathrm{rpm}$ at the design shaft power of $2244 \mathrm{~kW}$ [91]. The rotational speed of the propeller is automatically set by an electronic control unit as a function of the flight segment and throttle. The controller has the additional tasks of changing the blade pitch (including reversing and feathering conditions), protecting the propeller-turboshaft system from dangerous conditions such as overspeed. It can also automatically synchronizes the propellers' phases to minimize the noise impact.

Filippone [90] indicates that when the controller is not able to modify the pitch to match the torque, the rotational speed of the propeller is reduced. This is the case in climb and cruise where the rotational speed is reduced to $82 \%$ (984rpm) of the maximum value. This result is in accordance with equation 4.9 where the maximum rotational speed at cruise conditions (M=0.41 at FL180) is estimated at $963 \mathrm{rpm}$ when the maximum tip Mach number is set to 0.76 to account for the high blade sweep.

Figure 5.1b shows the results obtained by Filippone [90] for the propeller efficiency as a function of advance ratio and blade pitch. These are the results of a tool using the Blade Element Method (BEM) approach with corrections for the convergence of highly loaded propellers. Actual experimental results could not be found for this type of propeller, so this study was chosen as the next best thing for validation purposes. It can be noted that by appropriately varying the blade pitch, the propeller efficiency can be kept nearly constant at flight speeds typical for climb (170 kts) and cruise (254 kts at FL180). The maximum values of the efficiency at each blade pitch angle are used as validation points in figure 5.2.

Figure 5.2 shows the results of the Actuator Disk Theory model used to compute the propeller efficiency in 


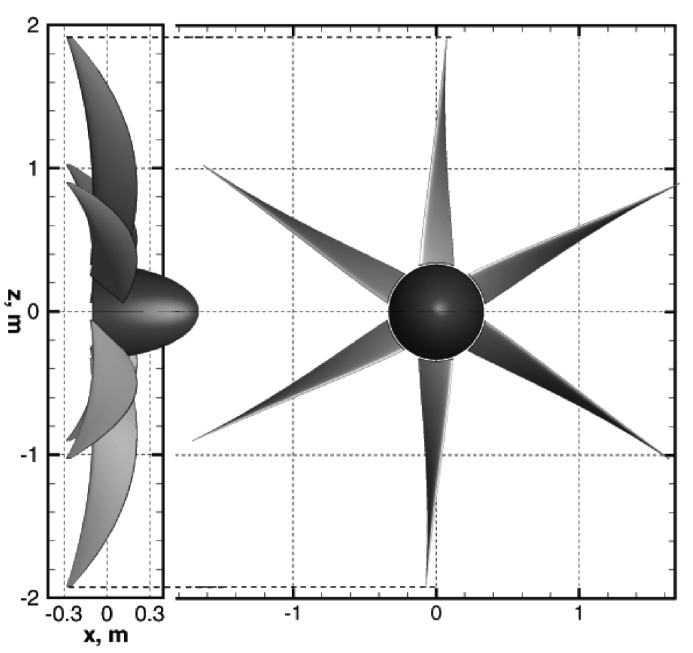

(a) Reconstruction of Hamilton F568 propeller used on ATR72 turbo-prop aircraft [91].

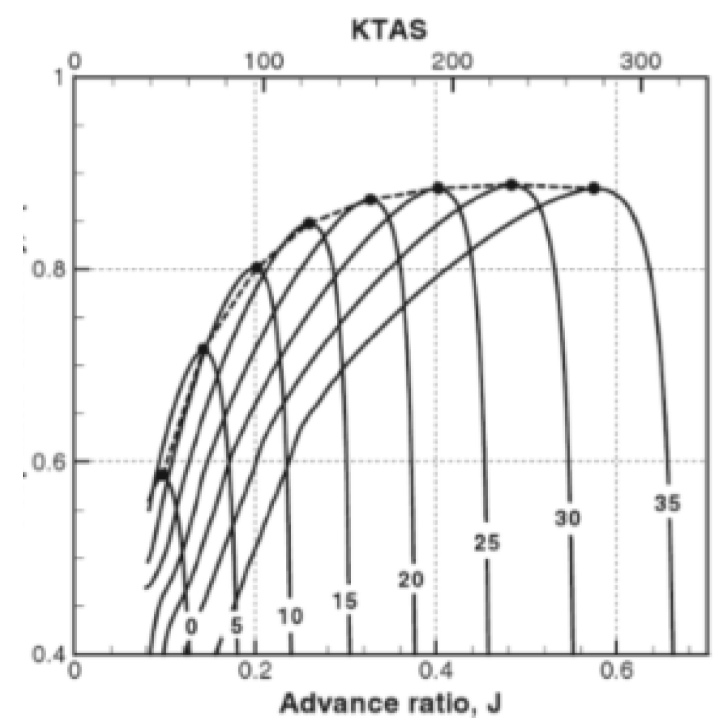

(b) F568 propeller efficiency as function of advance ratio J and blade pitch [90].

Figure 5.1: F568 propeller validation data from reconstruction of prof. Filippone [90, 91].

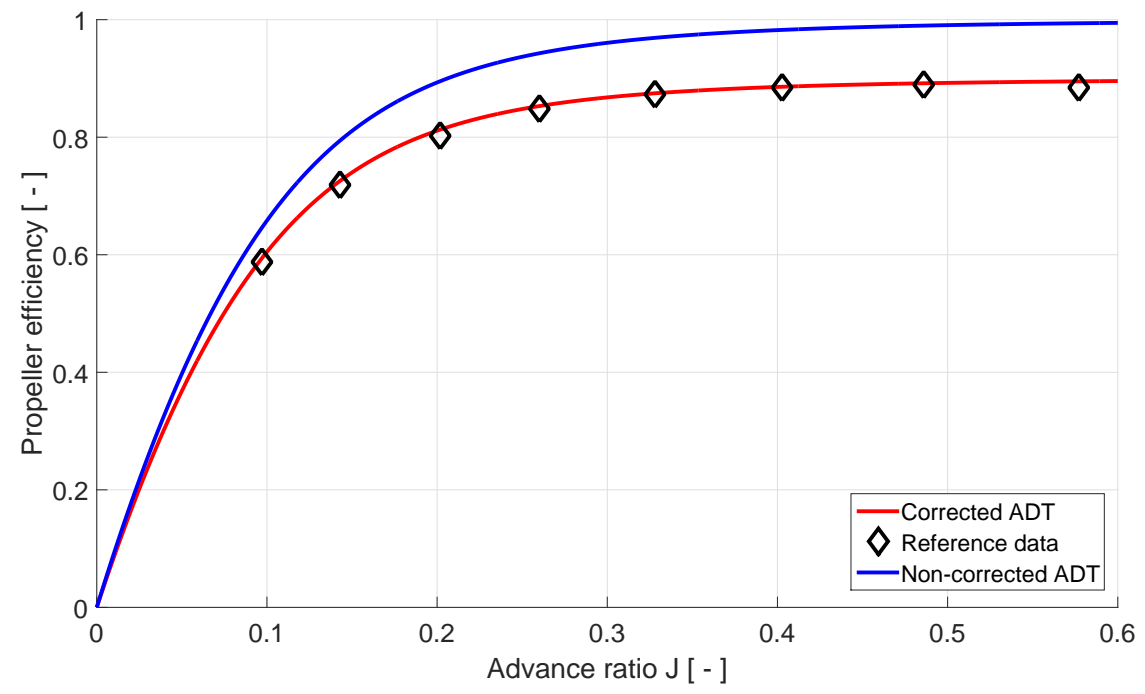

Figure 5.2: Flow chart of the proposed methodology for hybrid electric aircraft sizing.

this study. The blue curve represents the theoretical result as estimated from equation 4.5 where no correction factors are applied. The corrected propeller efficiency, in red, is obtained through the calibration of the correction factor. A value of 0.88 shows good accordance with the reference data and it in line with the expected overestimation of the ADT theory (10 to 15\%).

The reference study does not report efficiency estimations for advance ratios below 0.1 . For a propeller rotational speed of $1200 \mathrm{rpm}$ and diameter of $3.93 \mathrm{~m}$, the flight speed for this advance ratio is $49.4 \mathrm{~m} / \mathrm{s}$, just above the stall speed in landing configuration $(47 \mathrm{~m} / \mathrm{s})$ for the ATR72 [92]:

$$
V_{s, L}=\sqrt{\frac{M T O M \cdot g}{\frac{1}{2} \rho S C_{L, \text { max }}}}=\sqrt{\frac{23000 \mathrm{~kg} \cdot 9.81 \mathrm{~m} / \mathrm{s}^{2}}{0.5 \cdot 1.225 \mathrm{~kg} / \mathrm{m}^{3} \cdot 61 \mathrm{~m}^{2} \cdot 2.73}} \tilde{=} 47 \mathrm{~m} / \mathrm{s}
$$

Therefore, it is not possible to check the validity of the propeller model for the ground-based mission segments. These segments (taxi, take-off and landing) make up a small part of the mission so the uncertainty of 
the model in this range should not have a large impact on the overall energy consumption estimation.

Another limitation of the implemented propeller model is that the efficiency keeps increasing with the advance ratio approaching the limit set by the correction coefficient. As shown in figure 5.1a, the efficiency quickly drops as the velocity increases and the blades are at maximum pitch angle. This phenomenon is not of interest in this study as it is assumed that the propeller is sized with correct combination of aerodynamic and geometric proprieties to remain efficient even at the highest advance ratios encountered during the design mission.

\subsubsection{ELECTRIC MOTOR}

The electric motor model proposed in [68] is highly parametrized requiring only seven parameters to fully define the efficiency map and operational envelope. The seven parameters used in this validation are: the rated power, the maximum shaft speed, the maximum efficiency (i.e. a technology factor) and the coefficients $k_{0}, k_{P}, k_{Q}$ and $k_{\omega}$ identifying the point of optimal operation on the torque-speed graph. Two power machines have been chosen as references for the validation of the model.

The first is a $250 \mathrm{~kW}$ electric motor produced by $\mathrm{UQM}^{1}$ characterized by a maximum torque of $900 \mathrm{Nm}$ and a shaft speed of $5500 \mathrm{rpm}$. Seen as the rated power is around a tenth of the ATR72 mounted turbo-shaft engine, the motor could be used to supplement additional power to the engine when mounted in a parallel configuration, or directly connected to a small propeller is a distributed series architecture.

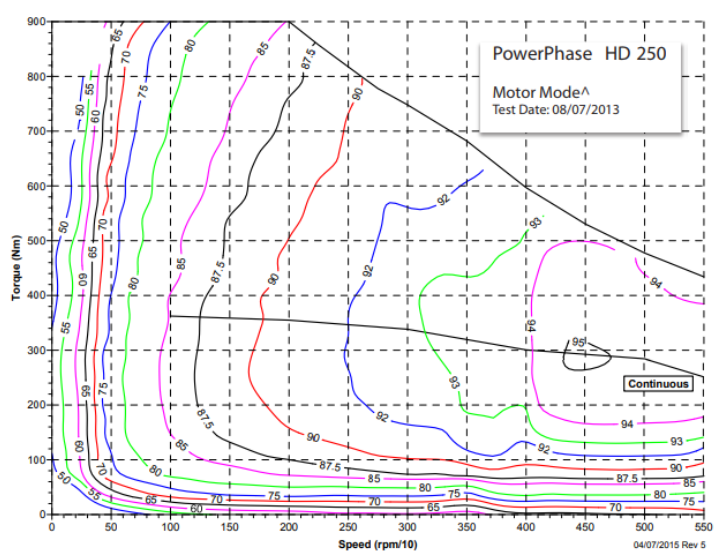

(a) Torque-speed operational map of UQM PowerPhase HD 250 with iso-efficiency lines.

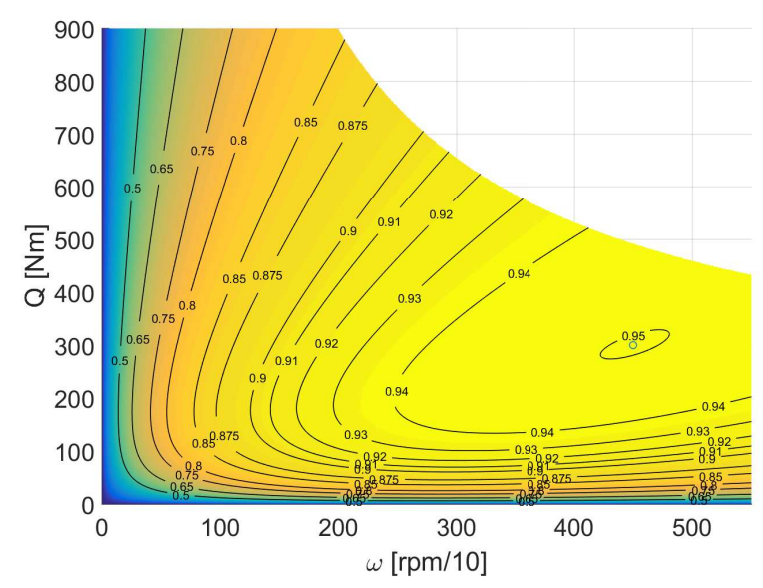

(b) Efficiency map obtained with electric motor model.

Figure 5.3: $250 \mathrm{~kW}$ electric motor operation and efficiency charts comparison.

Figure 5.3a show the performance chart published by the producer of the motor. It can be noticed that the point of optimal use is for rather high shaft speed and low torque. As the torque that the motor can generate depends on the current running through the armature, the optimum shows that, for a given power requirement, the losses are lowest when the applied voltage is higher (i.e. less current related losses). Overall, the performance maps show how an electric machine remains highly efficient throughout its working range. The efficiency only drops for lower shaft speeds and torque.

Assuming the same values of rated power and maximum shaft speed ( $250 \mathrm{~kW}$ and $5500 \mathrm{rpm}$ ), the electric motor coefficients $\left[\eta_{\max }, k_{0}, k_{P}, k_{Q}, k_{\omega}\right]$ are set to $[0.95,1,1.8,3,1.2]$ to obtain the performance map in figure 5.3b. The resulting torque-speed chart is remarkably similar to the experimental results of figure 5.3a.

A difference in the maximum power curve is however observed. The experimental results show an almost linear boundary between maximum torque and shaft speed where the method prediction has a hyperbolic curve. This happens because the motor model assumes a constant shaft power line where the experimental power changes between $190 \mathrm{~kW}$ at the maximum torque point and $250 \mathrm{~kW}$ at the maximum speed one. The

\footnotetext{
${ }^{1}$ Company Website, "Powerphase HD 250" , 2015. URL: https://www.neweagle.net/support/wiki/ProductDocumentation/EV Software and Hardware/Electric Motors/UQM/PowerPhase. Accessed on: 17/04/2018
} 
lower rated power at maximum torque could be explained by cooling capabilities that cannot effectively remove all the heat generated due to the high current related losses. Regarding the efficiency predictions, the model results follow the same trend as the iso-lines of figure 5.3a. Small differences are noted in the shape of the iso-lines as the model prediction is slightly more optimistic closer to the rated power curve.

The second reference machine is an electric motor proposed by NASA as part of their SUGAR Volt design in collaboration with Boeing [11]. This electric motor has a similar rotational speed as the previously shown motor $(4500 \mathrm{rpm}$ ) but it is rated for a much higher shaft power of $8000 \mathrm{hp}$ (almost $6 \mathrm{MW}$ ). This power makes it too large for use on an ATR72 like design using a parallel propulsion architecture. However, a similarly powerful machine could be used as an electric generator in a series architecture with mission requirements similar to the baseline aircraft.

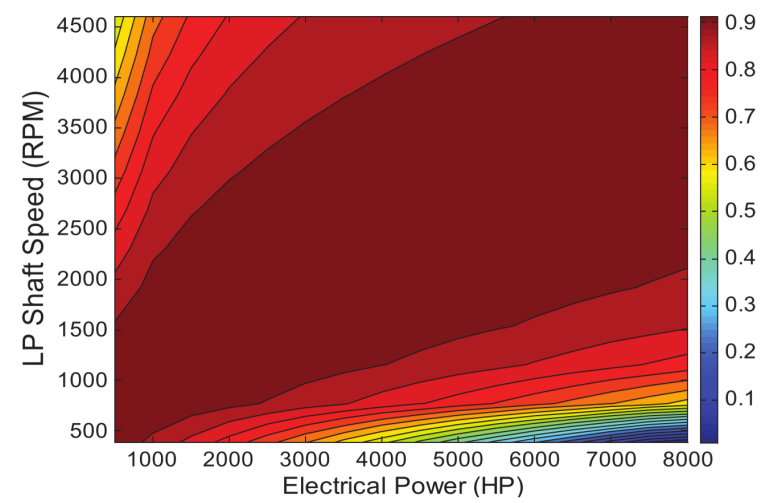

(a) Performance map of high power electric motor used in NASA study on hybrid electric aircraft [28].

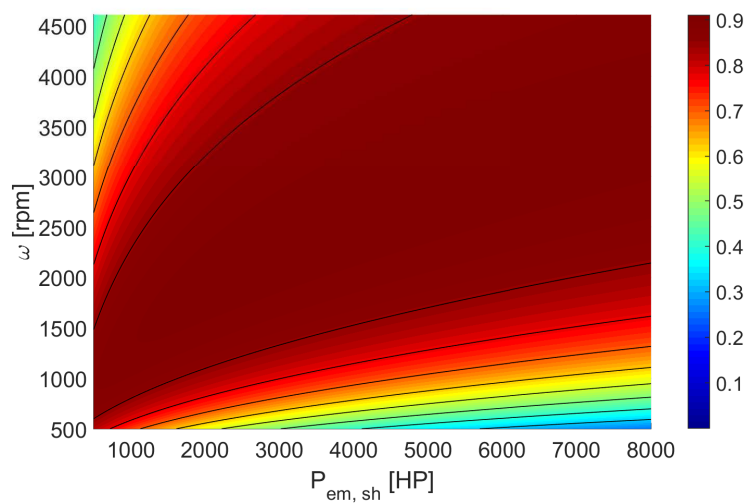

(b) Resulting electric motor performance map using method proposed in [68].

Figure 5.4: Motor efficiency as function of shaft speed and power characteristics of 8000hp machine proposed for the Boeing's SUGAR Volt design $[11,28]$.

The machine performances of report [28] are reported with the aid of a speed-power chart as shown in figure 5.4a. The results of this study have been obtained with a higher-level method taking into consideration motor dimensions and material specific losses. Similarly to the UBQ 250, this motor shows high efficiency values throughout the working envelope with rapid decreases near the low-speed, high-power range (i.e. high torque and current) and toward the no-load operation point characterized by low-shaft power and high rotational speeds.

The electric motor model results are shown in figure $5.4 \mathrm{~b}$ beside the NASA performance map to facilitate the comparison. The model coefficients $\left[\eta_{\max }, k_{0}, k_{P}, k_{Q}, k_{\omega}\right]$ used in this case are set to $[0.91,0.5,2,2,1.75]$. Similarly, to the previous comparison, the efficiency results show a good accordance between the reference and estimated efficiency maps. The only notable differences are seen in the prediction of the high-load lowspeed characteristics where the computed efficiency remains overall higher than the reference study. This might indicate that the used method is optimistic when evaluating the losses due to current in the motor. As the generator can be directly attached to the low-pressure spool of a turbo-shaft, the rotational speed would remain high to avoid engine flame-out or compressor surge.

Overall, the implemented model for the motor efficiency and operational boundaries show accordance with data found in literature. The model seems to remain valid for a large range of rating power allowing its use in the design space exploration.

\subsubsection{CABLING SYSTEM}

Power transmission lines have efficiency values close to $100 \%$ as the only source of loss considered in DC applications comes from heat generation due to the conductor internal resistance. Therefore, their efficiency variation is expected to not have a significant impact on the result of the sizing process. On the other hand, depending on their cross-section and system voltage, they can add non-negligible mass to the propulsion system. 


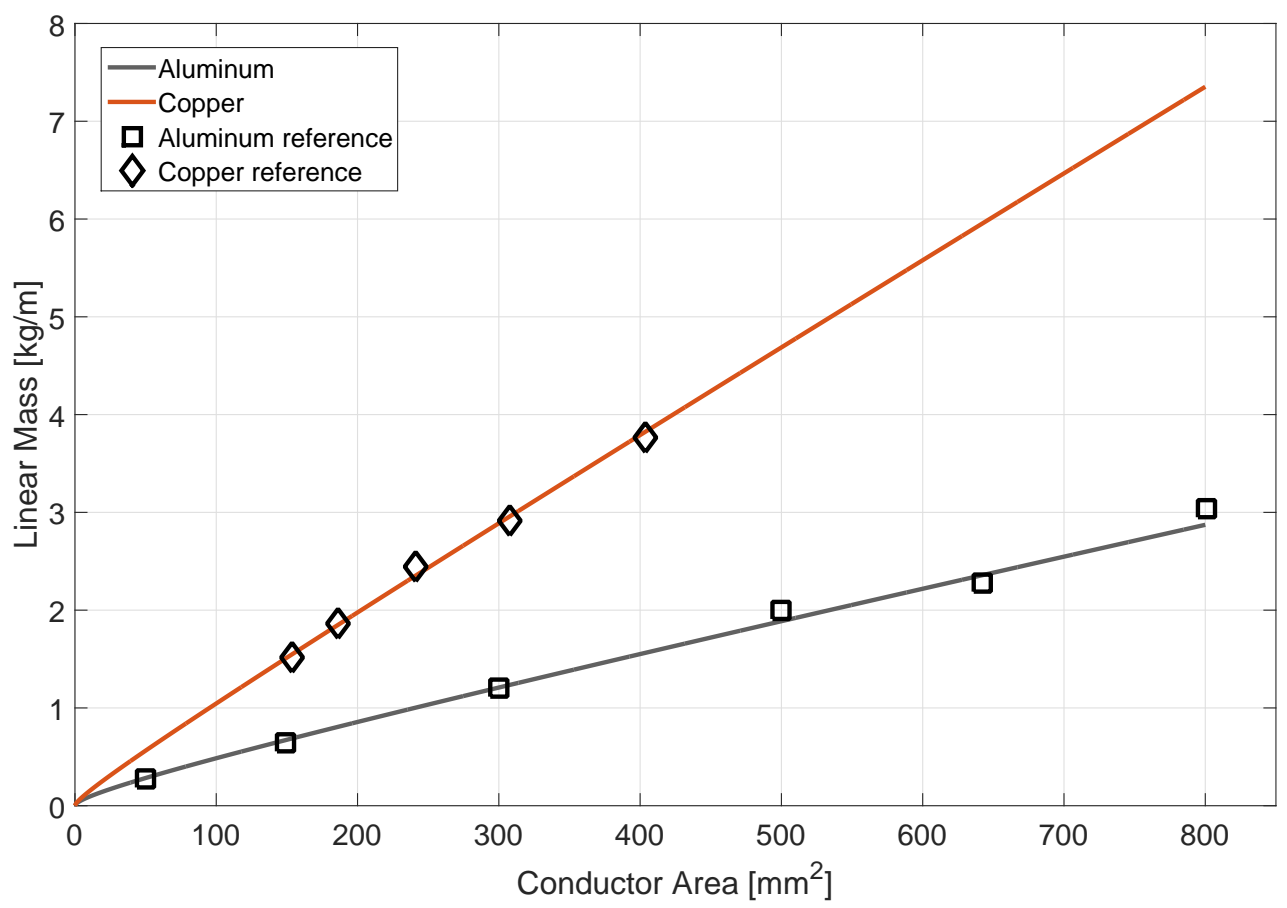

Figure 5.5: Cable specific mass as function of its cross-section. Reference data as reported in reference [64].

Figure 5.5 reports the cable linear mass estimations using the method proposed in [64] alongside manufacturer data ${ }^{2}$ for high power cables rated for voltages between 1 and $30 \mathrm{kV}$. These cables are made for groundbased power installations however, since no aircraft is yet using the amount of electric power at the required voltages expected in this study, they are the only source of reference material. It is possible that aircraft-grade cables could have different specific weights, but it is not clear if the trend would be toward lighter (use of better technologies, lighter insulators) or heavier (increase sheath and insulation to improve safety). Nevertheless, conventional cable technology is mature, so no large differences are expected.

The results have been obtained by varying the power requirement between $1 \mathrm{~kW}$ and $2 \mathrm{MW}$ at a fixed system voltage of $1.5 \mathrm{kV}$. The model shows good prediction capabilities for the cable linear specific mass on the entire range of conductor cross-sections considered. The weight advantage of the aluminium conductor is clear both from the results different slopes and from the absence of reference points for the copper cable past a certain diameter highlighting the higher efficiency does not compensate other key parameters such as cost and weight.

\subsubsection{ELECTROCHEMicAl CELL}

To validate the cell model, its predictions are compared to experimental data of the Panasonic NCR20700A battery cell ${ }^{3}$. This cell is characterized by a rated capacity of $3100 \mathrm{mAh}$, nominal voltage of $3.6 \mathrm{~V}$ and a mass of around $60 \mathrm{~g}$ resulting in a gravimetric specific energy of $190 \mathrm{Wh} / \mathrm{kg}$.

Figure 5.6 shows the model results overlay on to the experimental data. The cell discharge curve is reported for four increasing discharge rates. The reference results show that with increasing discharge currents the cell voltage is overall lower as expected from equation 4.37 . Outside the fully charged and discharged area the voltage drops linearly with the degree of discharge of the cell. Around a state of charge of $10 \%$ the voltage quickly drops.

The cell is modelled with a $4.2 \mathrm{~V}$ open-circuit voltage at $100 \%$ SOC and a cell internal resistance of $0.016 \Omega$.

\footnotetext{
${ }^{2}$ Nexans S.A., "Power Cables 1-30 kV," 2010, URL: www.nexans.de. Accessed on: 20/04/2018.

${ }^{3}$ Company website. "Panasonic NCR20700A Data Sheet", URL: https://akkuplus.de/mediafiles/Datenblatt/Panasonic/Panasonic NCR20700A.pdf, Accessed on: 20/04/2018
} 


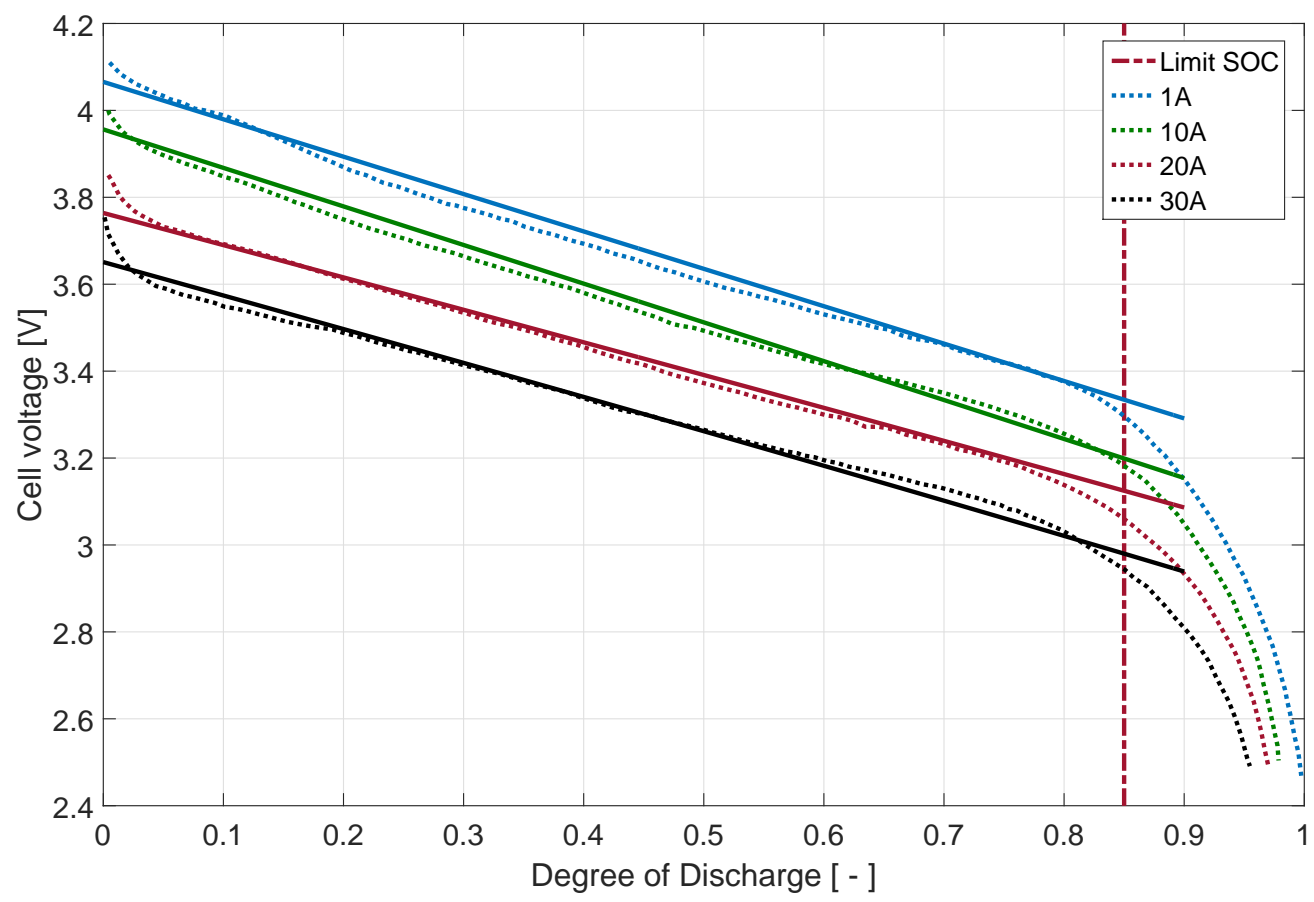

Figure 5.6: Discharge curve of Li-ion cell[93] and battery cell model results.

The cut-off voltage has been set to $83 \%$ of the fully charged potential. To avoid damages to the battery cell, the SOC lower boundary is set to $15 \%$. The results of the model show a good match with the experimental data in the DoD range considered. The predicted voltage starts lower than the actual reference fully charged potential as the exponential area is not modelled. The internal resistance increases due to concentration issues as the cell nears full discharge state but since the model assumes a constant internal resistance, the difference grows rapidly in this range. The cell would be used in this range only in an emergency scenario (e.g. diversion to alternate airport) while during normal operation the battery would not be discharged below the SOC limit to avoid shortening its life so uncertainty over the mission can change depending on the SOC assumed at the end of the hold/diversion. A end-of-diversion SOC of $20 \%$ is assumed in this study to improve the method accuracy and as an additional safety measure.

\subsection{BASELINE DESIGN VALIDATION}

With the components of the hybrid electric propulsive system validated, the method is used to design a baseline aircraft with conventional (i.e. fuel based) propulsion. This baseline design is used both as a further validation for the sizing loop and all the methods previously implemented in the design tool and as the reference point for the result analysis.

Studies found in literature tend to agree that the performance characteristics of the electric components required in a hybrid aircraft will lead to feasible and interesting (economically or ecologically) solutions only on smaller aircraft[94]. Even the most optimistic previsions of the battery specific energy cannot compare with the $12 \mathrm{kWh} / \mathrm{kg}$ offered by jet-fuel thus the proposed solutions become less and less interesting as the mission range increases. Moreover, as mentioned in chapter 4 , the electric motor specific power tends to drop as the rating increases after the cooling requirements become the constricting factor in the machine design. Therefore, studies indicate that by the year 2035, taken as reference for this study, hybrid electric propulsion could make sense only on aircraft with installed propulsive power in the order of 3-7 MW and with mission ranges below $2000 \mathrm{~km}$ [8].

The selected reference aircraft is the ATR72-600, a 70-passenger design with a total installed power of around $4 \mathrm{MW}$ and a harmonic range of $1500 \mathrm{~km}$. The aircraft has been chosen has it is widely successful in the 
turbo-prop regional market thanks to its range and payload capabilities that make it an interesting choice for regional routes. Moreover, the main design parameters and characteristics can be easily found in the literature as the ATR family has been used as a reference in a multitude of studies. This simplifies the baseline design validation and calibration and it has the added benefit of allowing the comparison of the results of the investigations into hybrid electric powertrain of this study with works that chose the same starting point such as $[64,87,95]$.

\subsubsection{POINT PERFORMANCE CONSTRAINTS}

The stating point in the design process proposed in this study is the definition of the constraint for the computation of power and wing loading values. The constraints are a combination of regulation requirements (FAR and CS25) that needs to be satisfied for safety reasons, and specifications usually derived from customer and market analysis expectations ${ }^{4}$. The constraints considered in this study are:

- Landing distance of $1067 \mathrm{~m}$ : this constraint is not affected by the installed power and results in a maximum allowable wing loading characterized by a vertical straight line on the constraint diagram as shown in figure 5.7 .

- Maximum span constraint of $36 \mathrm{~m}$ : as the study is interested in designing an aircraft able to compete in the regional market, the span limitation used in regional airports (ICAO code $\mathrm{C}$ ) should be used to limit the minimum wing loading. This constraint is represented by a vertical boundary in the constraint diagram. As the estimated MTOM of the design increases, this constraint could become larger than the maximum wing loading thus no-feasible design space would remain. A way to solve this issue is to allow the aspect ratio to decrease once the minimum and maximum wing loading coincide. A lower aspect ratio leads to a stubbier wing that is usually lighter but less aerodynamically efficient as the liftinduced tip vortexes become stronger. This has detrimental effects on the power requirements of high lift segments (take-off, climb and landing).

- Take-off distance of $1333 \mathrm{~m}$.

- Minimum Rate Of Climb at ceiling altitude: $30 \mathrm{~m} / \mathrm{min}$ at $3000 \mathrm{~m}$ in OEI conditions.

- FAR25/CS25: These are climb gradient requirements during critical flight phases as reported in reference [57]. Some of these constraints needs to be satisfied in OEI conditions so the value of the climb gradient changes depending on the number of propulsive units.

- Time to climb to cruise altitude: 17.5 minutes at $5400 \mathrm{~m}$.

- Maximum cruise of 0.45 at cruise altitude.

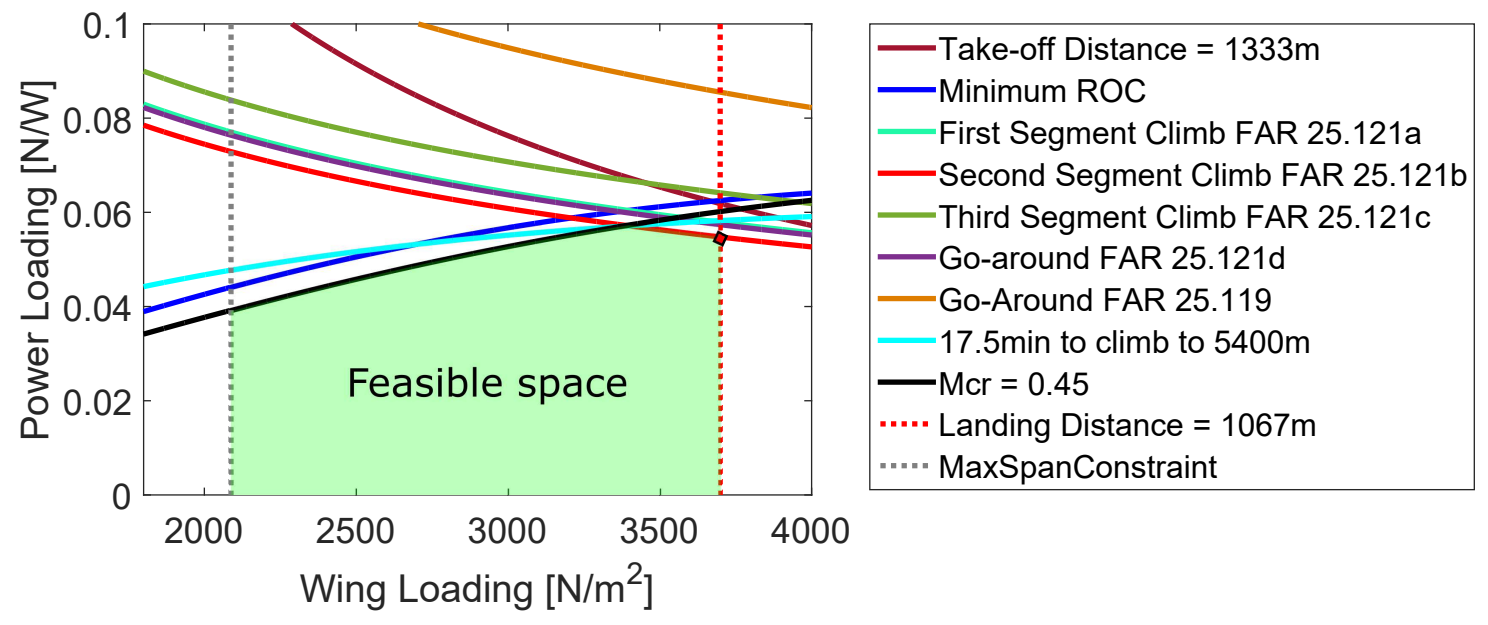

Figure 5.7: Baseline design power loading constraint diagram.

\footnotetext{
${ }^{4}$ ATR website brochure. "ATR Family", 2014, URL: http://www.atraircraft.com/products app/media/pdf/FAMILY septembre2014.pdf,
} Accessed on: 15/06/2018. 
Figure 5.7 shows the constraint diagram derived from the ATR72 requirements. The design space is delimited on the left by the maximum span constraint, on the right by the landing distance requirement and on the top by the power loading constraint, the cruise speed and second segment climb in OEI constraints. The design point can be chosen anywhere in this space, but the minimum weight is achieved for the highest combination of power and wing loading as their increase means smaller (and lighter) propulsion system and wing area. For the baseline design the active boundaries are the landing distance, resulting in a wing loading of approximately $3700 \mathrm{~N} / \mathrm{m}^{2}$, and the OEI climb gradient during the second segment climb resulting in a power loading of $0.055 \mathrm{~N} / \mathrm{W}$.

\subsubsection{MiSSION REQUIREMENTS}

Table 5.1 reports the harmonic mission requirements for the ATR72-600 as found in literature [92]. These mission inputs will be kept constant for the rest of this study in the HEP design space exploration and analysis.

\begin{tabular}{lcc}
\hline \multicolumn{3}{c}{ Harmonic Mission } \\
\hline Passengers & $70(@ 95 \mathrm{~kg} /$ PAX $)$ & $\mathrm{PAX}$ \\
Payload & 7500 & $\mathrm{~kg}$ \\
Cruise range & 1530 & $\mathrm{~km}$ \\
Cruise altitude & 5500 & $\mathrm{~m}$ \\
Cruise speed & 0.43 & $\mathrm{Mach}$ \\
& 500 & $\mathrm{~km} / \mathrm{h}$ \\
TOFL & 1333 & $\mathrm{~m}$ \\
LFL & 1067 & $\mathrm{~m}$ \\
Taxi & $4+4$ & $\mathrm{~min}$ \\
\hline \multicolumn{3}{c}{} \\
\hline Diversion range & 182 & $\mathrm{~km}$ \\
Diversion altitude & 3000 & $\mathrm{~m}$ \\
Diversion speed & 0.4 & $\mathrm{Mach}$ \\
Hold time & 470 & $\mathrm{~km} / \mathrm{h}$ \\
\hline
\end{tabular}

Table 5.1: Harmonic mission parameters for baseline design.

Figure 5.8 shows the change in altitude of the harmonic mission and diversion to the alternate airport in the top picture and the power requirements on the bottom one. The climb phase is carried out at constant CAS below $3000 \mathrm{~m}$ then the airspeed is increased up to the cruise Mach number of 0.43 . The cruise segment is carried out at constant altitude as the optimization of the altitude as the aircraft weight changes depends on the degree of hybridization between battery and fuel energy. Albeit it could be an interesting result to analyse, it was decided to keep the altitude constant to simplify the comparison between architectures. The descent is carried out at a constant rate of descent of $1000 \mathrm{fpm}$ as suggested in [80]. Take-off and landing fields are assumed to be at sea-level with ISA temperature.

Regarding the power requirements of figure 5.8, blue is used for the total propulsive power, black for the shaft power (i.e. corrected by the propeller efficiency) and red for the equivalent shaft power at sea-level (i.e. corrected by the power lapse of the PPU). The take-off segment is done at full throttle, while for the mission climb the power requirement is reduced at $90 \%$. The diversion climb is done at $96 \%$ of the take-off power to reach the diversion altitude faster. Taxi, cruise, descent and landing power requirements are computed as explained in chapter 4 .

\subsubsection{BASELINE RESULTS}

Using the implemented method and the inputs discussed previously, the baseline design is sized. The results of the sizing loop are compared to the reference values of the ATR72-600 in table 5.2. Overall, the computed values are in good accordance with the reference data. The sizing tool tends to slightly underestimate the fuel consumption leading also to a lower maximum take-off mass. The largest differences are in the power loading 

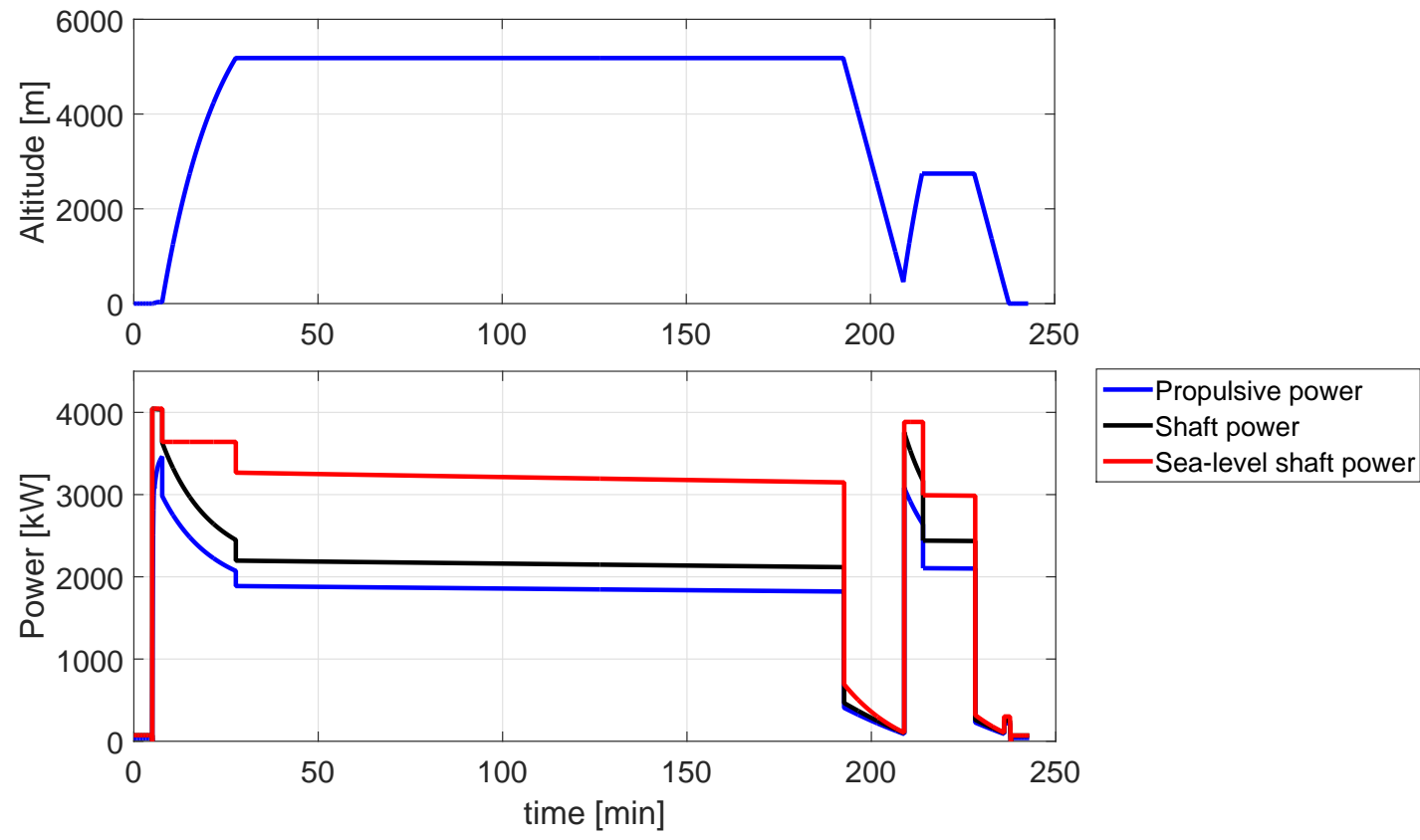

Figure 5.8: Baseline design altitude and power as function of mission time.

constraint result at $-1.2 \%$ and the total shaft power at $-1.3 \%$. this could contribute to the underestimation of the fuel consumption as the fuel flow estimation is directly sensitive to the power loading as the fuel map is normalized by the engine installed power.

\begin{tabular}{lc|ccc} 
& & Reference & Baseline & \% difference \\
\hline MTOM & {$[\mathrm{kg}]$} & 23000 & 22800 & -0.9 \\
OEM & {$[\mathrm{kg}]$} & 13500 & 13500 & +0.2 \\
Payload & {$[\mathrm{kg}]$} & 7500 & 7500 & 0.0 \\
Mission fuel mass & {$[\mathrm{kg}]$} & 2000 & 1980 & -1.1 \\
PW constraint & {$[\mathrm{kW} / \mathrm{kg}]$} & 0.18 & 0.18 & -1.2 \\
Wing loading & {$\left[\mathrm{kg} / \mathrm{m}^{\wedge} 2\right]$} & 377 & 378 & +0.3 \\
Total shaft power & {$[\mathrm{MW}]$} & 4.10 & 4.05 & -1.3 \\
Total mission energy & {$[\mathrm{kWh}]$} & 23800 & 23500 & -1.1
\end{tabular}

Table 5.2: Reference and baseline conventional design.

Finally, figure 5.9 shows the baseline design three view drawing overlain on an ATR72-600 drawing showing that also the external geometry of the aircraft is in good accordance with the expectations. The largest differences are seen in the vertical tail dimensions and position. With this the validation of the propulsion system components and overall method is deemed satisfactory and the baseline results can be used as reference for the results discussion. Values for the constraint and mission requirements are kept invariant for the results presented in chapter ch: results. 


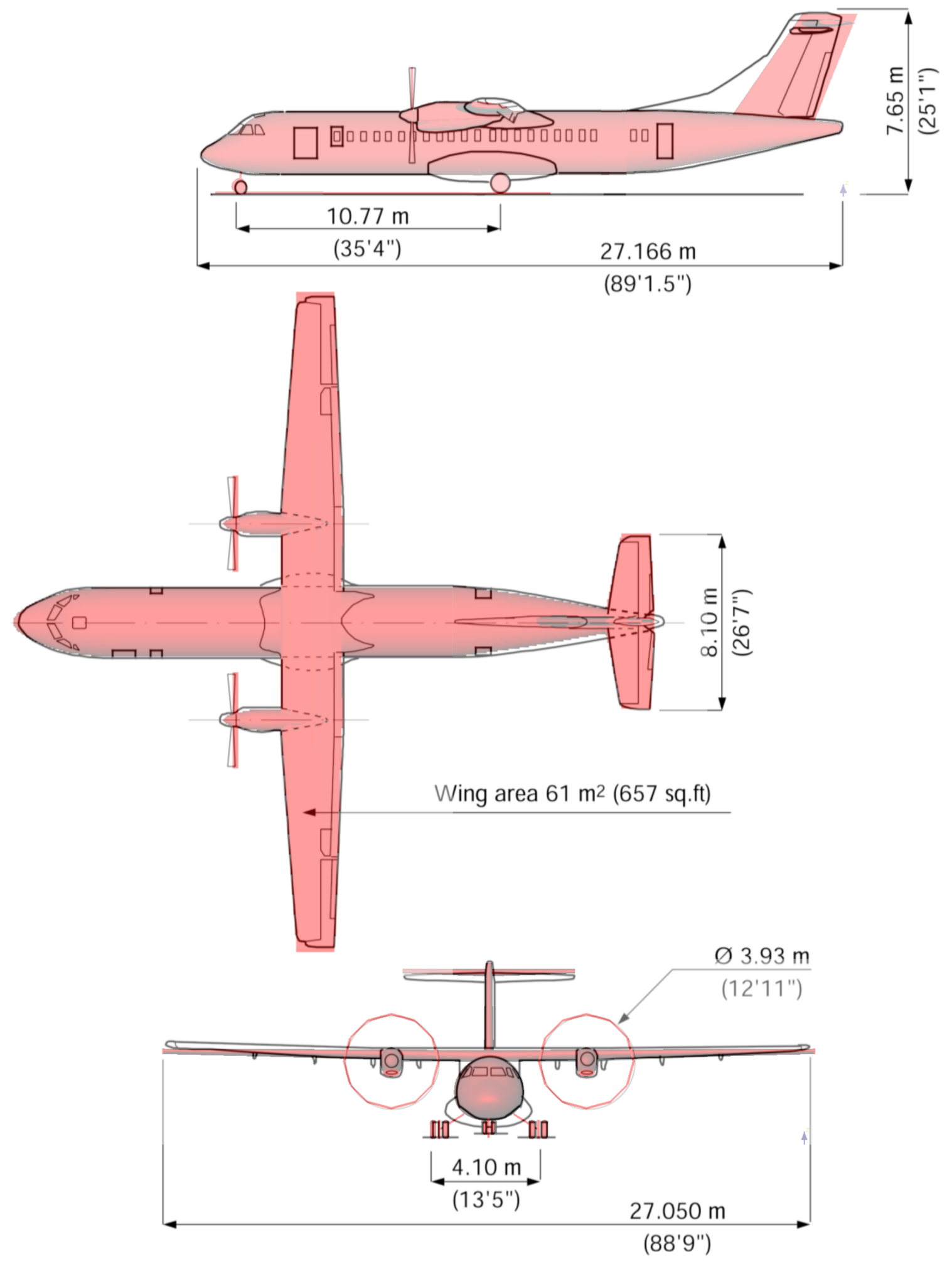

Figure 5.9: ATR72 3-view drawing [92] with overlay of baseline design result in red. 


\section{6}

\section{RESULTS}

In this chapter the method presented in 4 is used to analyse the differences deriving in terms of key performance indicators when hybrid electric powertrains are used in substitution of a conventional propulsive system. The constraints and harmonic mission presented in chapter 5 are kept unchanged and used in the sizing process to allow a fair comparison between the resulting designs and the baseline aircraft.

The results are subdivided in four main sections. Firstly, three HEP architectures are presented and discussed with attention given to the assumptions used in the sizing process. Then, a design space exploration for variable control approaches is performed for three levels of technology of the electric components allowing the individuation of optimal design points. Starting from these results, the three architectures are further improved and an in-depth discussion of the constraint diagram, point mass analysis and KPIs results in presented in the third section. Finally, a sensitivity study is carried out to assess which components and characteristics are the most critical for the hybrid electric designs.

\subsection{Hybrid Electric Powertrain architectures}

In this section, three distinct HEP architectures have been chosen among the ones described in chapter 3 to cover the most promising solutions. These architectures have also been chosen as their HEP system can be fully described with only one of the three control parameters as further explained in their respective sections: 6.1.1, 6.1.2 and 6.1.3. Architecture schematics to better understand the differences between these configurations are shown in appendix B.

\subsubsection{Parallel architecture}

The first design considered is defined by a conventional two propeller configuration driven by parallel hybrid PPUs each containing a turbo-shaft engine, an electric motor and a gearbox connecting them to the propeller. Figure 6.1 shows the external geometry of the design. As expected, from the exterior, the design does not show any difference from a conventional turbo-prop. However, this aircraft has a more complex propulsive system when compared to the baseline design as electrochemical batteries, a high-power cabling system and electric modules are needed to enable the electric side of the HEP.

Due to the conventional disposition and number of propulsive units, no benefits in terms of aero propulsion interactions are expected and the constraint analysis results will be affected by the power lapse correction only. As the motor and engine are mechanically coupled, no improvements to the gas-turbine specific fuel consumption are considered for this architecture. The electric motors are directly connected to the engine power turbine as the higher rotational speed allows for a smaller motor for a given power rating.

The propellers are sized with the same disk loading $\left(170 \mathrm{~kW} / \mathrm{m}^{2}\right)$ as the baseline design that is then kept constant throughout the design space exploration so that the propeller area changes alongside the shaft power of the PPU. The fuselage dimensions depend only on the passenger and internal volume requirements, that do not change with the variation of the degree of hybridization. However, the same variation has a large impact 


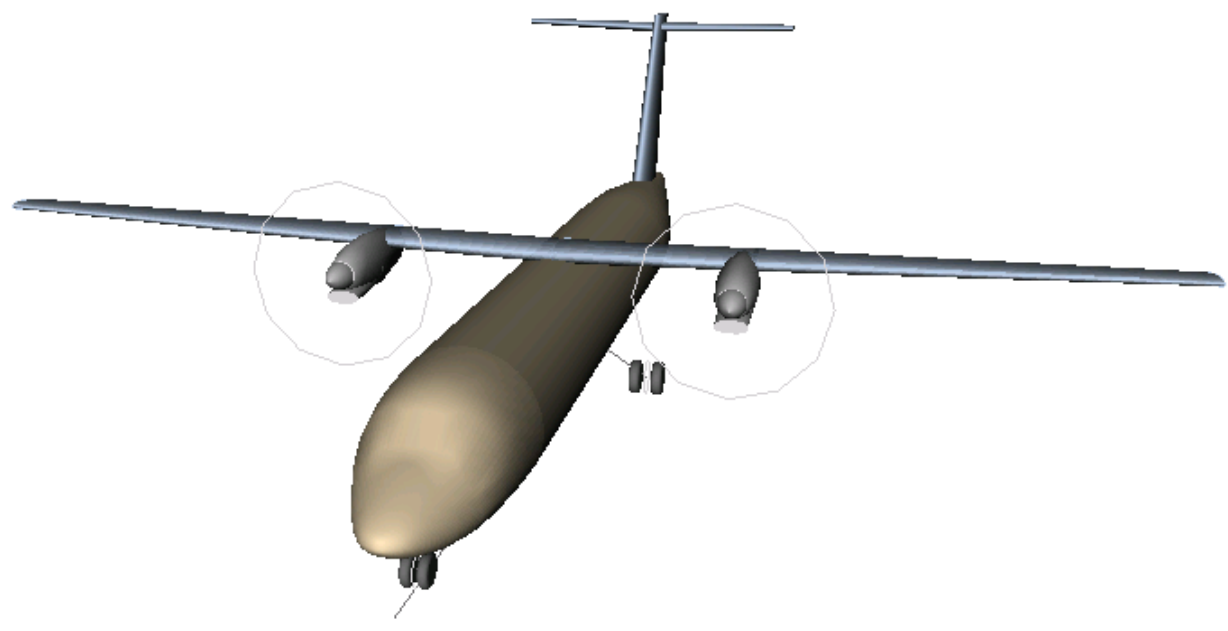

Figure 6.1: Parallel aircraft external propulsive system configuration

on the aircraft mass and installed power thus for a constant disk loading, the propeller diameter increases with the shaft power. If the diameter increases excessively, the safety margins between the propeller tip and ground might not be respected any more. If the wing is not moved (or the PPU position with respect to it), two ways to solve the issue are to increase the landing gear height or the disk loading. The first has a detrimental effect on the design mass while the latter has detrimental effects on the propulsive efficiency. To simplify the result analysis, this change is not implemented under the assumption that it would have small impact on the overall design. The assumption is then tested in the sensitivity analysis of chapter 6.4.

The gearboxes in the parallel PPUs are the only power nodes present in this architecture as there is no turboelectric generator providing electric power to the motors. The electric power ratio, $\varphi$, is thus set to 1 (i.e. electric power completely sourced from the battery packs). Moreover, since only two PPU are present, it is expected that they will be symmetrical in terms of power rating meaning that the propulsive power share, $\Theta$, of each PPU will remain equal to 0.5 for the entirety of the mission. Therefore, the HEP can be fully described with the use of the shaft power ratio, $\phi$, that will be varied between 0 (propeller driven by engine only) to 1 (propeller driven by motor only). An example schematic of a parallel architecture is shown in figure B.1.

\subsubsection{PARALLEL/SERIES HYBRID ARCHITECTURE}

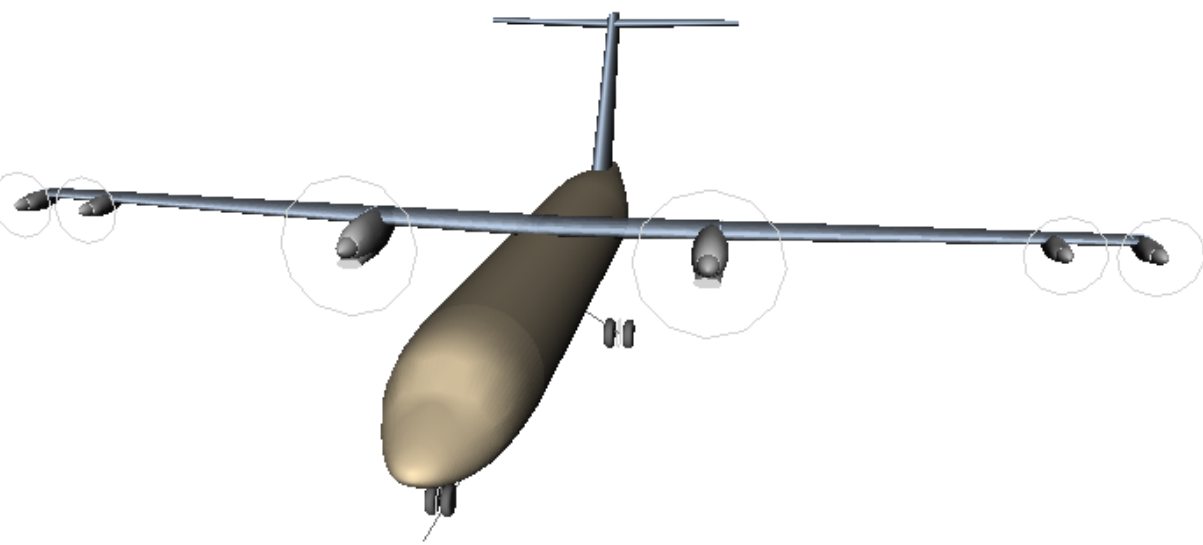

Figure 6.2: Series/parallel aircraft external propulsive system configuration.

The second architecture taken into consideration is a parallel/series hybrid with a low degree of propulsion distribution. Similar designs have been proposed by DLR [95], NASA [1] and other researchers [87] as an 
interesting concept to leverage the good scalability and controllability of electric motors.

The internal propulsion system is composed by a battery pack in the cargo bay supplying energy to the PMAD and the four electric motors mounted at the tip of the wing. The fuel-based system uses wing-tanks for the energy storage and two turbo-shaft engines providing power to the larger inboard propellers. The resulting design is visible in figure 6.2. This is the same configuration studied in [87, 95] and it differs from the NASA design [1] in that NASA opted to mount two parallel PPUs at the wing tip and make the inboard power units fully electric with foldable propellers. It is assumed that the electric motors are less likely to fail when compared to the more fragile turbo-machinery component in the parallel PPU. This means that the parallel power machine is more critical and mounting it at the tip of the wing would exacerbate the problems due to OEI conditions (large yaw moment due to non-symmetric thrust and drag). In contrast, the DLR design uses two sets of electric motors that are independently controlled so that in case of a motor failure, the aircraft can be more easily stabilised. A disadvantage of this disposition is that the cable system will be much longer and heavier due to the battery position in the centre of the cargo hold.

An advantage of the external PPUs configuration is that the tip propellers can be designed to improve the aerodynamic characteristic of the aircraft. References $[96,97]$ indicate that placing a propeller power unit at the wing tip has three main effects:

- Wing effect on propeller: the wing induces a change in angle of attack seen by the propeller blades as a consequence of lift generation. This can have the effect of increasing the produced thrust for a set torque requirement thus effectively increasing the propeller efficiency.

- Propeller effect on wing: when a wing produces lift, a vortex system is shed at the tip. This vortex induces a downwash effect on the wing requiring a higher angle of attack to compensate the effect thus tilting the aerodynamic resultant backwards. This phenomenon is known as induced drag. By placing a propeller that rotates in the opposite direction of the vortex, its strength is reduced thus attenuating the lift induced drag.

- End plate effect: the motor nacelle provides some blockage to the flow field around the wing akin to an end-plate placed at the wing extremity. The limitations of the interaction between upper and lower surface flows partially reduces the strength of the tip vortexes.

These are complex effects that depend on a multitude of characteristics among which there are the wing shape, its aspect ratio, propeller thrust, diameter, angle of attack and lift distribution shape and magnitude $[78,98]$. Modelling these phenomena is outside the scope of the thesis. However, the possible improvements to aircraft aerodynamics is the main driver for this configuration so it would not make sense to completely omit the effect. The benefit magnitude highly depends on the strength of the tip vortexes, so it will be higher for low-aspect ratio wing and high-lift scenarios. Reference [1] takes results from a low-aspect ratio study [97] and compares it to the results for a high-aspect ratio wing for low speed missions [58] and deriving an $18 \%$ improvement to the propeller efficiency. This assumption is also used in this study by applying the improvement to the outboard propellers using the efficiency corrector factor $k_{\eta_{P}}$.

The parallel/series configuration defined above has the characteristic that the fuel-based sub-system and the electric one is completely separated from one another. Therefore, the inboard power unit is modelled as a parallel PPU where the shaft power ratio remains constantly at 0 . Similarly, the out-board propellers are driven by motors that receive power from the battery packs only, thus the electric power ratio is fixed at 1 . Therefore, the only remaining way to control the degree of hybridization is to use the propulsive power share during the segment. As for the parallel design, the high-power segments and lesser demanding ones are grouped in two separate groups and their respective control parameters are varied in bulk during the design space investigation. The free control parameter is the power share of the fuel-based PPU while the electric propellers' control depends on it. Therefore a $\Theta=1$ means that the thrust is fully produced by the fuel-based engines, while $\Theta=0$ indicates fully electric propulsion. Figure B.2 shows the schematic for the parallel hybrid architecture used in this study.

\subsubsection{Distributed SERIES ARchitecture}

The third architecture chosen for the design space exploration is a series HEP characterized by distributed propulsion along the entirety of the wing span. This propeller configuration is often presented alongside hy- 


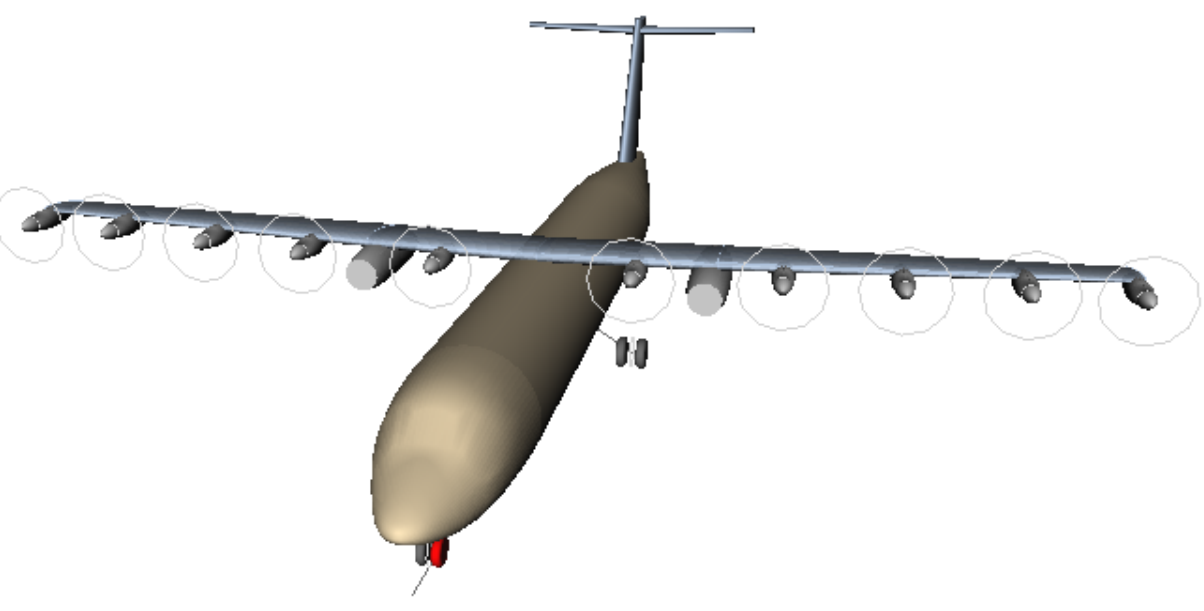

Figure 6.3: Series aircraft external propulsive system configuration.

brid or full-electric powertrain as turbo-machinery engines lose a substantial amount of thermal efficiency as they are scaled down. On the other hand, electric motors can be scaled almost without any penalty and the distribution of the power requirement further helps with their cooling requirements. This type of configuration is usually known as Distributed Electric Propulsion (DEP) and similar designs as the one depicted in figure 6.3 have been previously studied (e.g. [58, 59, 95, 99]). These studies usually focus on the aerodynamic and operation characteristics as the aero-propulsive coupling typical of the configuration render them quite complex. Less focus is given to the internal structure of the powertrain as most of the time it is assumed that the aircraft will be fully electric [99].

The proposed design makes use of ten identical electric motors distributed along the wing with constant spacing, beside a small gap at around $30 \%$ of the span to provide the required space for the generator-turbo shaft system. This disposition is chosen to maximize the surface of the wing that is immersed in the propellers slipstream. Propellers produce thrust by axially accelerating a mass of air. If a lifting surface is immersed in this stream, it will be subjected to a higher dynamic pressure thus producing a larger lift and profile drag [59]. This increase is more pronounced during low-speed segments as the relative increase in dynamic pressure is larger. This interaction can be leveraged to reduce the wing area as it is usually sized for landing requirements. A smaller wing is lighter, produces less drag in high-speed conditions and costs less making this choice highly interesting. Drawbacks are that the lift is coupled to the thrust setting, making the control of the aircraft more difficult and the design less safe in case of propulsion system failures.

In this study, this phenomenon is modelled through correction coefficients used in the computation of the aircraft lift polar. As the effect is stronger in high-thrust low-speed conditions, the take-off airfoil lift coefficient, $C_{l, \text { max }}$, has the largest change at $+15 \%$. Landing has the problem that thrust cannot be too large otherwise descent and deceleration during approach cannot be achieved so a lower increase of $+10 \%$ is assumed. A small $2 \%$ improvement in airfoil lift is assumed for high-speed segments. Moreover, to account for the more turbulent flow hitting the wing, an increase of $10 \%$ of the airfoil drag coefficient is also used.

The internal structure of the hybrid propulsive system is composed by the ten fully electric PPUs that are then connected to the PMAD through electric cables. The PMAD receives electric power from two distinct sources: batteries and electric generators. Two electric generators are mounted below the wing to be close to the propulsors. Other possible positions could be above the wing [95] or at the aft of the fuselage although worse airflow or heavier cable system would result from these configurations respectively. It is assumed that the generators are directly coupled to the free turbine of the turbo-shaft engine so that no gearbox is required. The generator-engine system is oversized by a factor of two to ensure that electric power can be provided even in the eventuality of an OEI condition.

The thrust is split uniformly among the ten propellers, so the propulsive share ratio is kept constant at a value of 0.1 for each PPU. Moreover, the propeller is driven by fully electric PPUs so also the shaft power ratio remains constantly equal to 1 throughout the mission. The only remaining degree of freedom for the PPU control is then the electric power ratio, $\varphi$, that is varied between 0 (electricity produced by generators only) 
to 1 (electricity provided entirely by batteries). The complex internal architecture resulting for this HEP is shown in figure B.3. As for the other designs, to simplify the design space exploration, the control parameters are grouped by mission segment and are kept constant for their duration.

\subsection{DESIGN SPACE EXPLORATION}

In this chapter the aircraft sizing method developed as part of this thesis is applied to study and compare the impact of the propulsion system architecture on some key performance indicators. The HEP sizing method allows for the specification of the propulsive system controls at the segment level. These operation parameters have a large impact on the design result as they control the power and energy ratios between fuel and energy based propulsive sub-systems. By grouping the high-power segments (take-off, climb, diversion climb) together in one group and the others in a second one (taxi, cruise, descent, hold, diversion descent and landing), only two control inputs are defined so that a series of response surfaces of the KPIs can be shown. This design space exploration is useful to show the differences between architectures and to highlight trends as the power and energy degree of hybridization change as a function of the control strategy.

It is assumed that hybrid electric aircraft are highly sensitive to the technology maturity level achieved by the propulsive system components, in terms of specific power, energy and power conversion efficiency. To test this assumption, the design space exploration is repeated three times for three increasingly optimistic assumptions. The full definition of these assumptions is described in section 6.2.1.

\subsubsection{TECHNOLOGY MATURITY LEVEL ASSUMPTIONS}

Technology advancement of the components of the HEP have a large impact on the aircraft design. Moreover, depending on how well the electric components compare to the fuel-based system in terms of efficiency and specific weights, the optimal degrees of hybridization will also change. Therefore, the design space exploration is performed for three level of assumptions for the technology advancement of the electric components as summarized in table 6.1.

\begin{tabular}{lcccc}
\hline Input & Unit & Conservative & Optimistic & HTS technology \\
\hline$\varrho_{e m} / \varrho_{e g}$ & {$[\mathrm{~kW} / \mathrm{kg}]$} & $5 / 12$ & $9 / 20$ & $15 / 25$ \\
$\eta_{e m}$ & {$[\%]$} & 95 & 95 & 97 \\
\hline$\varrho_{e c} / \varrho_{c r y o}$ & {$[\mathrm{~kW} / \mathrm{kg}]$} & $9 / \mathrm{NA}$ & $15 / \mathrm{NA}$ & $16.5 / 5$ \\
$\eta_{e c} / \eta_{c r y o}$ & {$[\%]$} & $90 / \mathrm{NA}$ & $95 / \mathrm{NA}$ & $98.8 / 30$ \\
\hline$\rho_{c b, c o}$ & {$\left[\mathrm{~g} / \mathrm{cm}^{3}\right]$} & 3.3 & 3.3 & $5 \mathrm{~kg} / \mathrm{m}$ \\
$\sigma_{c b}$ & {$\left[\Omega \cdot \mathrm{mm}^{2} / \mathrm{m}\right]$} & $3.7 \cdot 10^{-2}$ & $3.7 \cdot 10^{-2}$ & $5 \mathrm{~W} / \mathrm{m}+P_{\text {loss, terminal }}$ \\
$\mathrm{j}$ & {$\left[A / \mathrm{mm}^{2}\right]$} & 2.5 & 2.5 & derived \\
\hline$\varrho_{b a t}$ & {$[\mathrm{~kW} / \mathrm{kg}]$} & 0.6 & 0.8 & 0.8 \\
$\epsilon_{b a t}$ & {$[\mathrm{Wh} / \mathrm{kg}]$} & 500 & 750 & 750 \\
$\eta_{b a t}$ & {$[\%]$} & 85 & 90 & 90 \\
\hline
\end{tabular}

Table 6.1: Technology level assumptions for the design space exploration.

All three assumptions are about an expected year entry into service of 2035-2040. The first technology expectation is the most conservative; the specific mass and efficiency of the motors and components are almost achievable with today's state of the art technology while the cable system is made of aluminium conductors to save as much weight as possible. The only components that has yet to achieve the conservative expectations is the battery, in terms of gravimetric energy density as a specific energy of $500 \mathrm{Wh} / \mathrm{kg}$ has been assumed. Looking back at the background research in chapter 2.1.5, it can be seen that this specific energy is already double what currently can be achieved with lithium-ion cells and it also exceeds the theoretical limits for those chemistry combinations. However, lithium-sulphur should be able to provide this level of performance and their technology level is quickly improving as found in the literature research.

The second technology level assumption is more optimistic in every regard. The expectations for the power modules and electric components are close to what is regarded as the technology limit without the use of super-conductive technologies. The battery specific energy is further improved to $750 \mathrm{Wh} / \mathrm{kg}$, a very optimistic value that could be achieved by lithium-air closed cycle batteries. 
Lastly, the improvements in efficiency and specific mass allowed using super conductive materials are studied with the last technology level assumption. Motors, generators and other components are sized with improved values as found in literature while the cabling system is changed to a constant linear mass and losses model. The battery characteristics are left unchanged. Moreover, two cryo-coolers are added in the hybrid electric powertrain to provide the cooling requirements. These modules are sized by taking as a reference the maximum power loss of motors, generators and cabling system, and a Carnot efficiency of $30 \%$.

To facilitate the reading of this chapter, the response surfaces resulting from the design space exploration for conservative and super conductive assumptions are moved to appendix A. The result analysis will nevertheless touch upon the trends observed as technology assumption becomes more and more optimistic.

\subsubsection{MAXIMUM TAKE-OFF MASS CHANGES}

\section{Relative MTOW change [\%] Optimistic assumption}

Right: Parallel architecture

Bottom: Parallel/Series hybrid architecture

Bottom right: Distributed series architecture
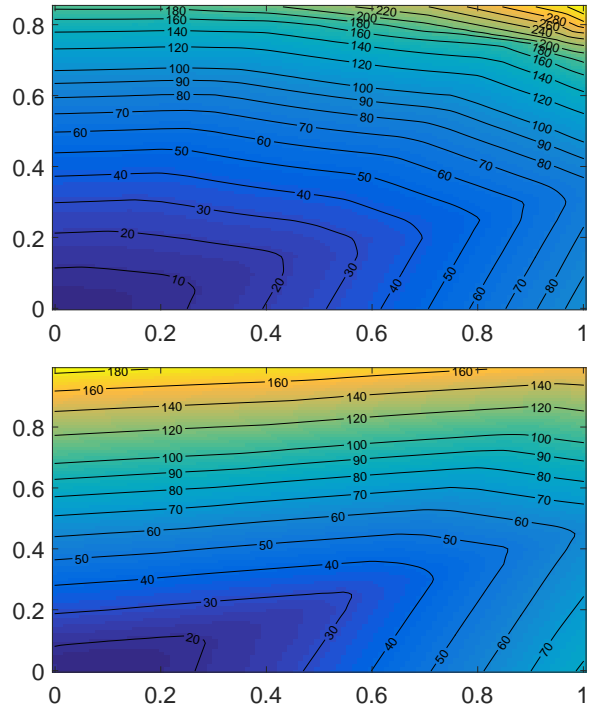

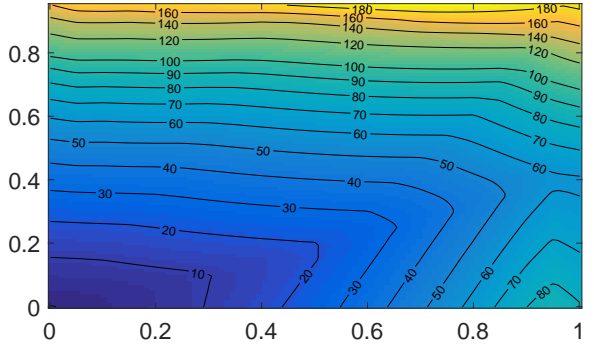

$\mathrm{DoH}_{\text {climb }}[\%]$

Figure 6.4: MTOW change as a function of the control parameters for three HEP architectures and optimistic assumption.

The first key performance indicator analysed is the MTOM of the design resulting by the combination of technology level, HEP control strategy and HEP architecture. Figures A.1, 6.4 and A.2 show the response surfaces resulting from the design space exploration with conservative, optimistic and HTS assumptions respectively. On the $\mathrm{x}$-axis there is the power Degree of Hybridization during the high-power segments while on the y-axis there is the low-power segments one. The DoH can be read as a general term to indicate the free control parameter that characterizes each configuration (i.e. a DoH $=1$ equals to $\phi=1$ for the parallel architecture, $\Theta$ $=0$ for the series/parallel HEP and $\varphi=1$ for the series configuration).

For all three level of technology considered, the reference mass of the design increases with the overall degree of hybridization. This change in mass is a consequence of the disadvantageous exchange of fuel for electricity as means of power source. As highlighted by the rapid MTOW increase as the $\mathrm{DoH}_{\text {cruise }}$ grows, this is due to the energy requirement at the battery and its very poor gravimetric energy density. On the other hand, the change in mass is weakly influenced by the increase of $\mathrm{DoH}_{\text {climb }}$ up to a certain point. This indicates that the exchange of propulsive fuel-based technologies for electric ones has little impact as their specific powers are comparable.

After a certain high-power segments DoH, each response surface shows a rapid increase in MTOW. This is explained by looking at figure 6.5 for the optimistic assumption and to figures A.3 and A.4 for the others. These figures show the electric energy surplus in the battery packs defined as the maximum usable energy contained in the batteries (i.e. not considering the minimum State of Charge set at 20\%) minus the energy consumed for the reference mission. It can be noticed that the for most of the design space the difference is zero. However, for low $\mathrm{DoH}_{\text {cruise }}$ and high $\mathrm{DoH}_{\text {climb }}$, the difference rapidly grows. This is due to the change 


\section{Electrical Energy surplus [\%] \\ Optimistic assumption}

Right: Parallel architecture

Bottom: Parallel/Series hybrid architecture

Bottom right: Distributed series architecture
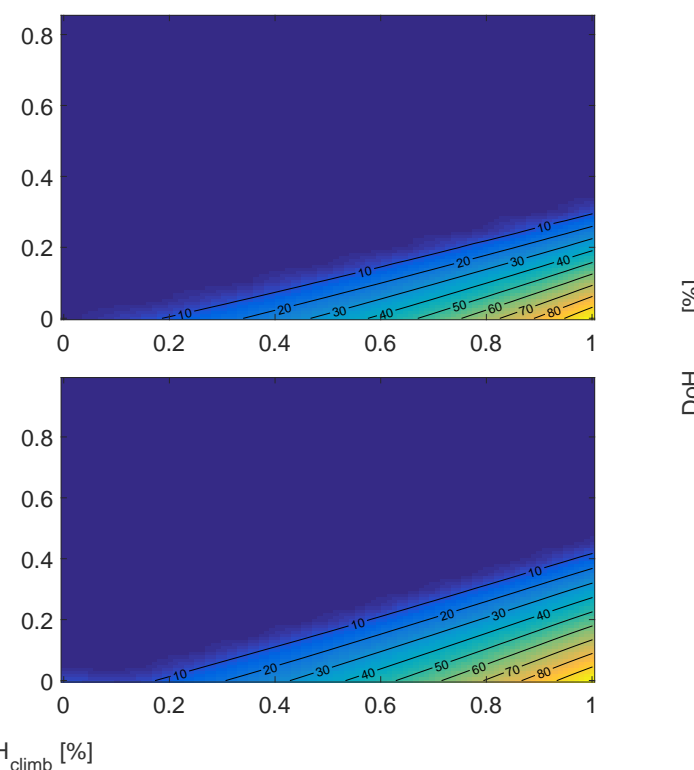

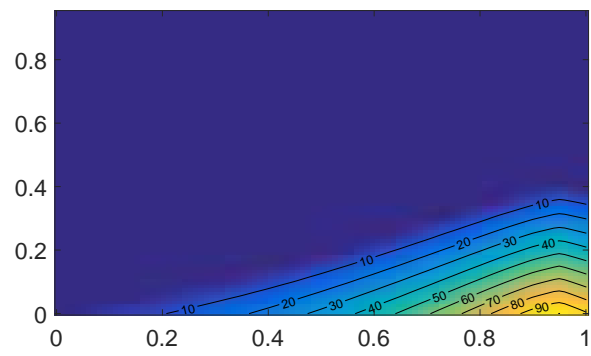

$\mathrm{DoH}_{\text {climb }}[\%]$

Figure 6.5: Response surface of the battery utilization as function of the control parameters.

in sizing requirement from energy to power. The boundary between these two regions is where the requirements coincide and in terms of MTOW they offer the largest electrification for the smallest increase.

Comparing the three levels of technology, the design mass shows a large decrease between the conservative and optimistic assumptions for all three architectures. Taking the point $\left[\mathrm{DoH}_{\text {climb }}, \mathrm{DoH} \mathrm{H}_{\text {cruise }}\right]=[0.2,0.2]$ as a reference, the parallel MTOW changes form $40 \%$ to $20 \%$ more than the baseline result, the parallel/series goes from $35 \%$ to $15 \%$ and the series shows the greatest reduction going from $80 \%$ to $30 \%$. The change in mass between the optimistic and futuristic (i.e. HTS technology) assumptions is more limited. This is in part due to the smaller change in performance parameters but also due to the addition of heavy systems (cryo-coolers and HTS cables).

The series design is overall the heaviest, but it is also the one that shows the largest sensitivity to the improving technology levels. This is because the thrust is produced only electrically requiring a heavier electric system for using and distributing the electricity.

\subsubsection{Mission FUEL AND TOTAL ENERGY}

As the energy degree of hybridization increases, the share of fuel to electric energy decreases. Thus, it would be expected that the mass of fuel required to fly the reference mission would decrease as the $\mathrm{DoH}_{\text {climb }}$ and DoH $H_{\text {cruise }}$ increase. This is partly confirmed for optimistic and HTS technology assumptions as shown in the response surfaces in figures 6.6 and A.6. For all three architectures, the assumption of high performance of electric components pushes the minimum fuel consumption towards the universally electric design $\left(\left[\right.\right.$ DoH $_{\text {climb }}$, Do $\left.\left.H_{\text {cruise }}\right]=[1,1]\right)$ that is in fact the global minimum for this parameter.

However, the trend is reversed if the degree of hybridization is increased by keeping one of the axes constant and moving the other, if the variable is the $\mathrm{DoH}_{\text {cruise }}$. This can be explained by the larger aircraft MTOW as the decrease of fuel mass due to electrification of the propulsion system is offset by the increase of power and energy required to fly the mission. This is further confirmed when looking at figure A.5 where the fuel consumption for the three architectures is mapped for the conservative technology level assumption. In this case, the trade-off between fuel and overall aircraft weight is so disadvantageous that the minimum of the surfaces falls closer to the conventional fully fuel-based design. Improvements of less than $10 \%$ can be achieved for this level of technology while the more optimistic ones allow for $100 \%$ reduction if the weight increase is not limited.

Comparing the three architectures shows that parallel and series/parallel have similar trends in fuel mass changes for all three technology assumptions. The DEP architecture has the main difference that the fuel 
Relative fuel mass change [\%]

Optimistic assumption

Right: Parallel architecture

Bottom: Parallel/Series hybrid architecture

Bottom right: Distributed series architecture
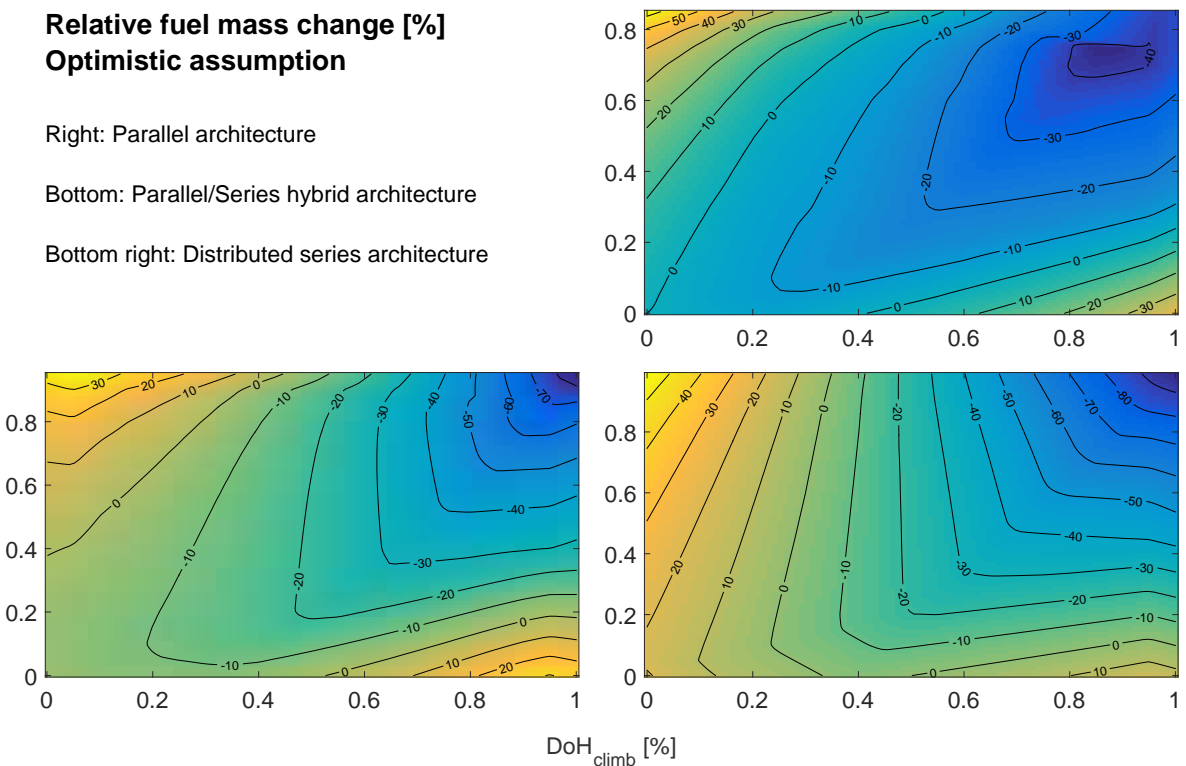

Figure 6.6: Design space results for fuel mass as function of the control parameters.

consumption is higher in the low DoH region even for conservative estimations. The design at $[0,0]$ represents the turbo-electric architecture defined by a fully electric propulsion group yet a fully fuel-based energy source. The overall worse performance of this architecture is due to the longer energy conversion chain that is required to convert the fuel power into electricity and then into shaft and propulsive power. The larger number of components are detrimental both to the aircraft mass and the system efficiency when compared to a conventional mechanical gearbox as used in the other two architectures. The fuel requirement for a turboelectric tends to the reference value for the most optimistic assumption thanks to the overall high efficiency of the superconductive system but the weight remains around $15 \%$ higher.

\section{Relative Energy change [\%] Optimistic assumption}

Right: Parallel architecture

Bottom: Parallel/Series hybrid architecture

Bottom right: Distributed series architecture

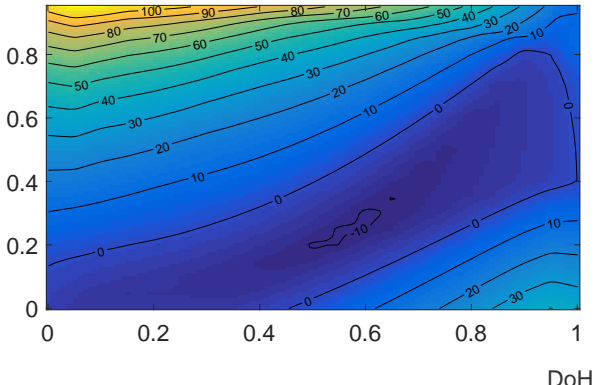

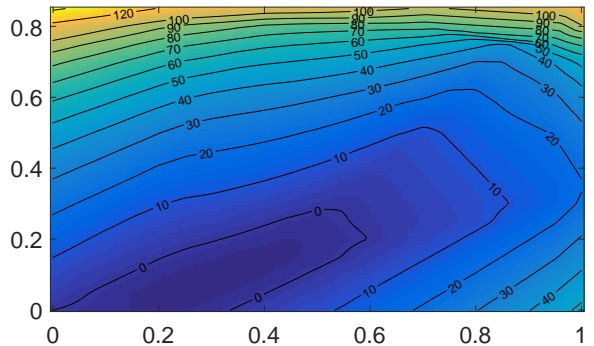

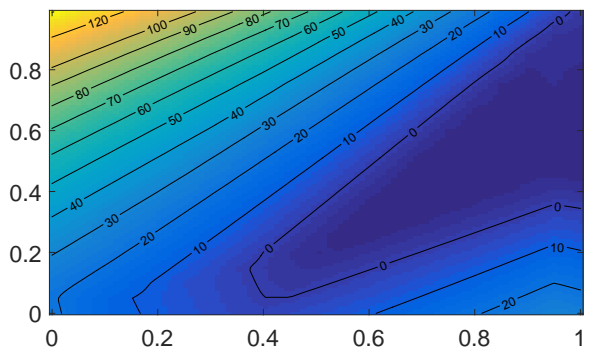

$\mathrm{DoH}_{\mathrm{climb}}[\%]$

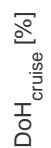

Figure 6.7: Total energy response surface for the three HEP architectures.

Figures A.7, 6.7 and A.8 show the results for the total energy change as a function of the HEP control parameters. The total energy accounts for both the fuel and electric energy stored in the tanks and battery packs respectively so it offers a more complete picture of the design effectiveness in carrying out the sizing mission. 
For low specific energy and power assumption, the energy result does not differ much from the fuel ones as the cost of a more electric propulsion system is not favourable. For the optimistic assumption, the minimum changes depending on the architecture. The parallel architecture shows that a low energy degree of hybridization in combination with a $20 \% \mathrm{DoH}_{\text {climb }}$ is the best choice for minimizing total energy consumption. For the series/parallel architecture, the minimum is moved to a more electric solution that is characterized by a $20 \%$ utilization of electricity in cruise and up to $55 \%$ during take-off and climb. Finally, the distributed series architecture shows a clear improvement when moving away from the turbo-electric solution and going toward a fully electric one. These trends are further magnified with the HTS technology assumption as the efficiency of the energy conversion is further improved.

For the lowest assumption, no reduction in terms of total energy are found in the first two architectures and a $35 \%$ increase characterizes the best DEP control strategy. Even for the better expectations, the total energy of the parallel solution cannot be reduced by more than $5 \%$ while for the others, improvements of $25 \%$ and $30 \%$ are possible. From these results, it can be concluded that the choice of architecture is highly influenced by the advancement of the electric components: parallel architectures are less sensitive to this change and offer the lightest design at the cost of smaller improvements, while the more electric architectures characterized by higher $\mathrm{DoH}_{P}$ offer more substantial improvements at the cost of more massive and complex designs.

\subsubsection{OVERALL EFFICIENCY, ESAR AND WELL-TO-PROPELLER EMISSIONS}

The last performance indicators considered in the design space exploration are the overall system efficiency, the Energy Specific Air Range and the $\mathrm{CO}_{2}$-equivalent emission impact considering well-to-propeller pollutants production.

\section{HEP overall efficiency change [\%] Optimistic assumption \\ Right: Parallel architecture \\ Bottom: Parallel/Series hybrid architecture \\ Bottom right: Distributed series architecture}
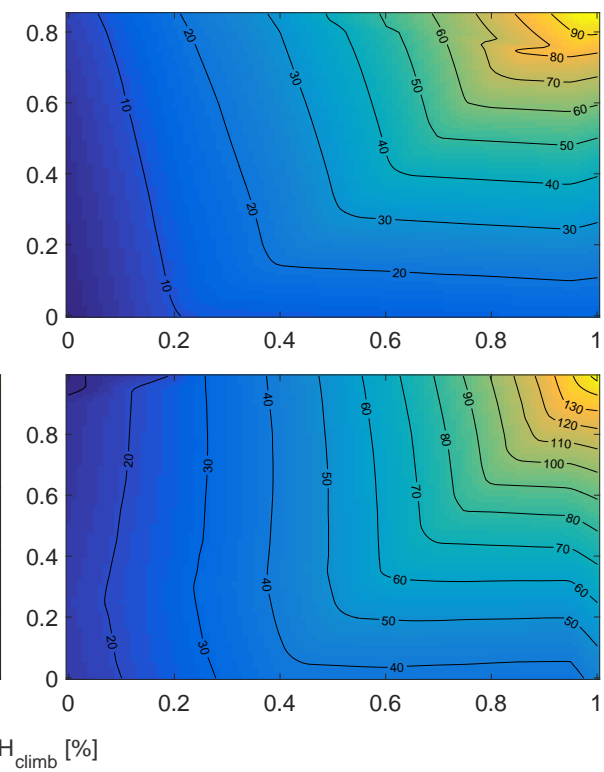

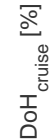

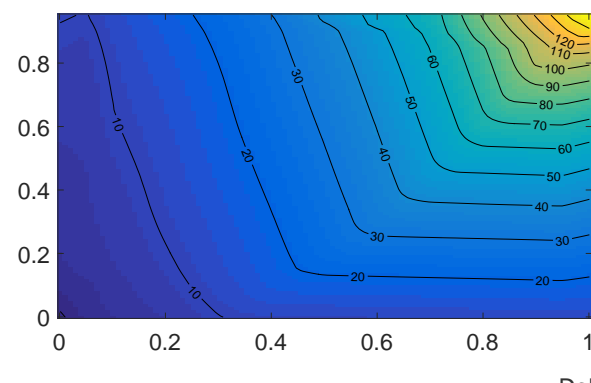

$\mathrm{DoH}_{\text {climb }}[\%]$

Figure 6.8: Response surfaces of propulsive system efficiency.

Figures A.9, 6.8 and A.10 report the change in system efficiency with the change of control parameters. The overall efficiency is defined as the time-based average of the propulsive power over the sum of fuel and electric power during the entire sizing mission. Two main trends are observed from these surfaces. Firstly, the efficiency monotonically increases with the increased electrification of the propulsive system regardless of architecture or technology level assumption. This verifies the assumption that an electric propulsive system is more efficient than a fuel based one. Secondly, the improvement is largest along the diagonal line that represents the battery sizing requirements boundary as seen in figure 6.5. This highlights the importance that the battery mass has on the overall design of hybrid electric aircraft as over-sizing in terms of energy or power nullifies the main improvement that could be derived from the electrification of the system. With fully electric HEP system, the reference efficiency of around $26 \%$ can be improved to a maximum of $36 \%, 62 \%$ and $75 \%$ for the three level of technologies studied. 
Using the Energy Specific Air Range, the combination of changes in overall efficiency, lift-to-drag ratio (i.e. aerodynamic efficiency) and mass can be analysed in a single parameter. Figures A.11, 6.9 and A.12 show the response surface of this KPI. For its definition, the larger the ESAR the better the design is considered as it will be able to fly the longest distance for a given amount of energy or conversely, fly the mission range with the least energy consumption.

\section{Relative ESAR change [\%] Optimistic assumption}

Right: Parallel architecture

Bottom: Parallel/Series hybrid architecture

Bottom right: Distributed series architecture
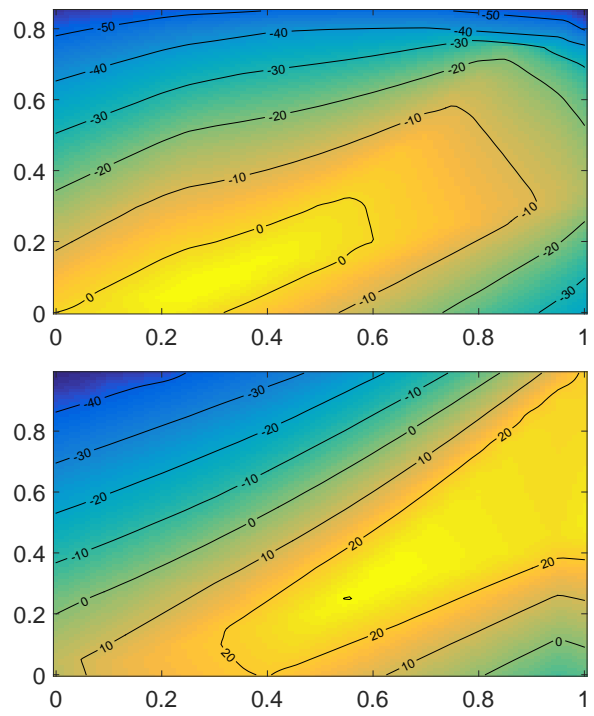

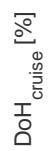

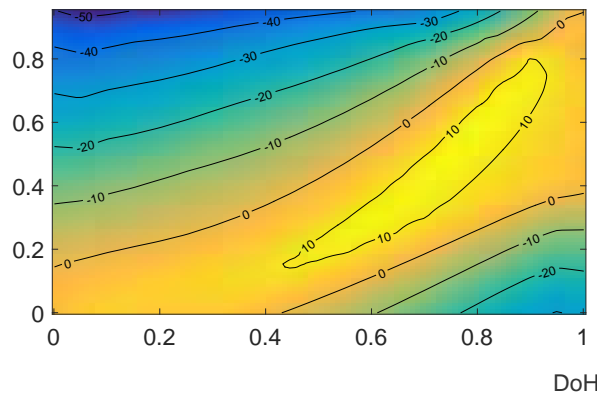

$\mathrm{DoH}_{\text {climb }}[\%]$

Figure 6.9: Energy Specific Air Range response surfaces.

For the conservative assumption shown in figure A.11, the tendency observed in the previous chapter is confirmed as the optimal designs of the three architectures are characterized by overall low degrees of hybridization with little to none use of electric power during cruise and between $10 \%$ and $40 \%$ electrification during take-off and climb for the series/parallel and series architectures respectively. For the super-conductive technologies expectations, the maximum ESAR for the series/parallel and series HEP is clearly the universally electric solution as it offers the largest increase of system efficiency that exceed the larger MTOW. On the other hand, the parallel solution shows that the maximum ESAR is constant along a diagonal line that connects the lowest fuel and the lowest energy points. this architecture also shows the lowest improvement in ESAR at around 5\%.

For the optimistic assumption shown in figure 6.9, the maximum ESAR for the parallel configuration is characterized by a $20 \%$ use of the electric system in climb and fully conventional cruise. This control combination slightly improves the ESAR by moving the sizing requirement of the fuel-based PPU from the climb/take off condition to the cruise one thus allowing savings in terms of fuel consumption in exchange for a slightly heavier design. The other two architectures reach their maximum ESAR for higher utilization of the electric system achieving increases of 15 and $30 \%$ with respect to the reference design. These points are used as starting values for the manual optimization of the designs that are then presented in more detail in chapter 6.3 and used in the sensitivity study of chapter 6.4.

Finally, the last KPI considered is the emission impact of the designs also taking into consideration the emissions due to electricity production and fuel refinement and transportation. As described in the methodology chapter 4.5, an increase of 0.05 and $0.43 \mathrm{kgCO}_{2} / \mathrm{kWh}$ are added to the flight emissions for fuel and electricity respectively. If electric energy is produced with a higher share of renewable energies, the response surfaces shown in figures A.13, 6.10 and A.14 would become the same as the results computed for the fuel mass change as the electric energy would not have any impact on the overall emissions.

No improvements to $\mathrm{CO}_{2}$ emissions can be obtained with any of the studied architectures if the more optimistic technology maturity levels are not achieved. In fact, the series architectures show a minimum increase of $40 \%$ emissions if the technology of batteries and motors does not grow beyond the conservative assumptions. With the more optimistic expectations, the well-to-propeller emissions can be reduced by about $5 \%$ for all architectures. Finally, for the most futuristic assumption, series/parallel and series designs could achieve 


\section{Relative Emissions change [\%] Optimistic assumption}

Right: Parallel architecture

Bottom: Parallel/Series hybrid architecture

Bottom right: Distributed series architecture
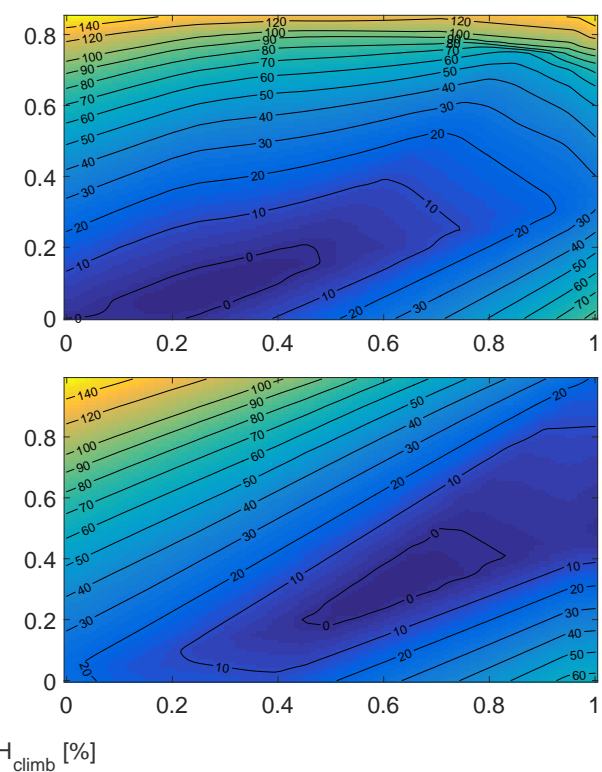

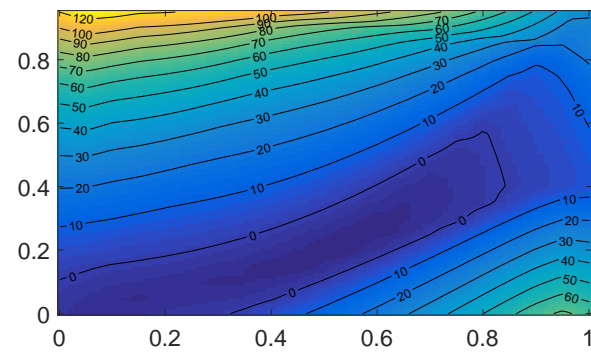

$\mathrm{DoH}_{\text {climb }}[\%]$

Figure 6.10: Emission $\mathrm{CO}_{2}$ equivalent impact response surfaces.

up to $15 \%$ reductions where the parallel one does not see significant improvements with respect to the previous expectation.

These improvements are rather small, but it is essential to consider that they are obtained with energy production emissions typical of today industrialized countries. It can be expected that by 2035 the share of renewable sources will be larger thus allowing more impactful reductions.

\subsection{Hybrid ELECTRIC AirCRAFT ANALYSIS}

With the design space exploration completed, one design point for each architecture is chosen and further improved in terms of performances. As the previous explorations has been carried out at constant control parameters for a group of segments, the resulting design is not optimized for its mission as part of the propulsion system could be oversized. The tool utilized for the sizing method implementation does not have a built-in optimizer, so a starting point is chosen from the design space and then improved manually by using linearly changing control parameters for each mission segments. Only the optimistic assumption is used for this analysis as little to no benefits can be found using the more conservative values and the super-conductive option have a large degree of uncertainty on the YEIS considered for this study.

The starting point for this manual optimization is found using the ESAR response surface as the parameter is useful in minimizing total energy requirements while still accounting for the increase of mass. The parallel architecture shows the best results for low battery utilization in cruise and around $25 \%$ electrification in flight. The series design has its maximum ESAR at around $55 \% D_{0} H_{\text {climb }}$ and $20 \% D o H_{\text {cruise }}$ indicating that a higher electrification is preferable for this design. Finally, the series/parallel architecture shows a flat top in its ESAR response surface. As the ESAR does not show significant changes in this area, the lowest degree of hybridization is chosen as it limits the increase of aircraft weight.

During the optimization process, it was noticed that the fuel and aircraft mass could be minimized near the starting point by making sure that the fuel-based system would work at a constant power during the mission. This control approach effectively uses the electric motors to move the sizing point of the conventional engines from the take-off segment to the cruise one. Consequently, the electric motor needs to provide power over a wider range but, as it is not affected by altitude and their efficiencies remain high over a wide envelope, this difference is relatively smaller when compared to the conventional case where to produce power at altitude the engines must be oversized to account for air density reduction. An example of this power shaving approach is shown in figure 6.11 where the electric motors are used to supply the excess power in take-off and climb only (upper figure) and as thrust generators also during the cruise to further decrease the fuel con- 


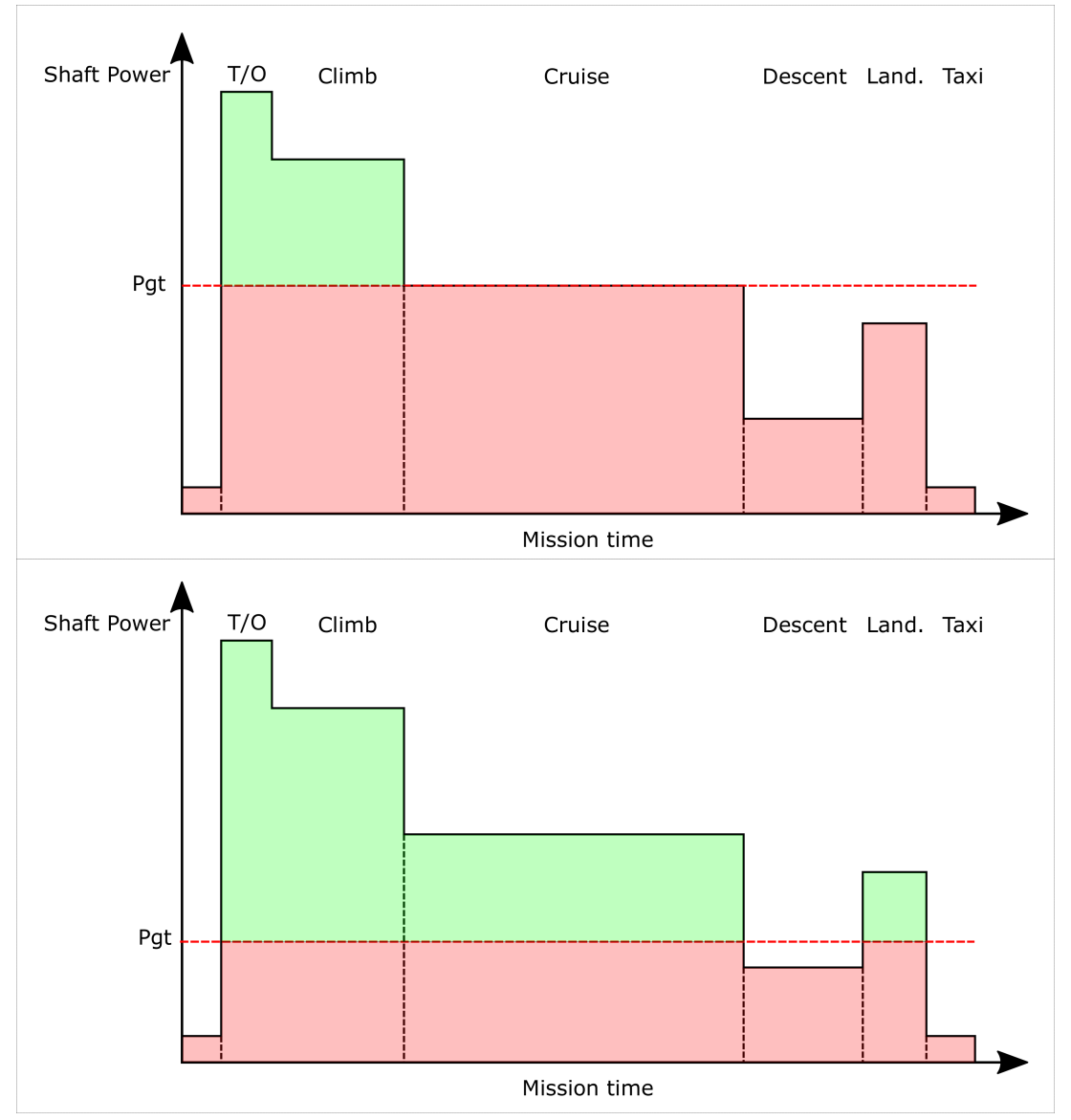

Figure 6.11: Power shaving control approaches with fully conventional cruise (up) and hybrid cruise (bottom).

sumption. As this approach offers better results than the starting points and it can be easily obtained with linearly changing controls along a mission segment, it was decided to use this control strategy for the final designs of the three architectures.

In the following sections, the results of the sizing process are discussed. Firstly, the constraint diagram is presented highlighting the sizing requirements and the choice of design point. Then the point mass analysis results are discussed concentrating on the power variation during the mission and the resulting energy consumption. Finally, the three designs are compared in terms of key performance indicators to indicate which architecture would be the most interesting for the given mission and YEIS.

\subsubsection{HEA CONSTRAINT ANALYSIS}

In this section, the constraint diagrams for the selected HEP architectures are shown and discussed. The constraints considered are the same used for the sizing of the reference aircraft where the climb-gradient regulation requirements are changed to the four engines case for the series/parallel and series architectures as they mount six and ten PPUs respectively. The results depicted in figures 6.12, 6.13 and 6.14 show a zoomedin image of the diagram that focuses on the design point and most constricting requirements only.

The sizing point on the constraint diagram is chosen as the combination of maximum wing and power loadings so that both the size of the wing and of the propulsive system can be minimized. It can happen that these two optimum values do not coincide so further information are used to differentiate between the two. In this study, this is done by plotting iso-MTOW (solid lines) and iso-energy (dashed lines) curves on the constraint diagram as suggested in [62] so that then the power and wing loadings combination that offers the largest energy savings for the lowest mass increase can be found.

Figure 6.12 shows the constraint diagram for the parallel HEP architecture. This design is characterized by 
two propulsive units so in the event of a failure, half the power is lost thus making the OEI second segment climb gradient requirement sizing. The design point is set at the intersection between this constraint and the landing requirement derived by the maximum lift capability of the aircraft. On the diagram, the intersection between the time-to-climb and OEI constraints offer a higher power loading (i.e. less installed power required) at the cost of a larger wing that could be chosen as a different design point. However, both the mass and energy curves indicate that the former intersection is the best choice.

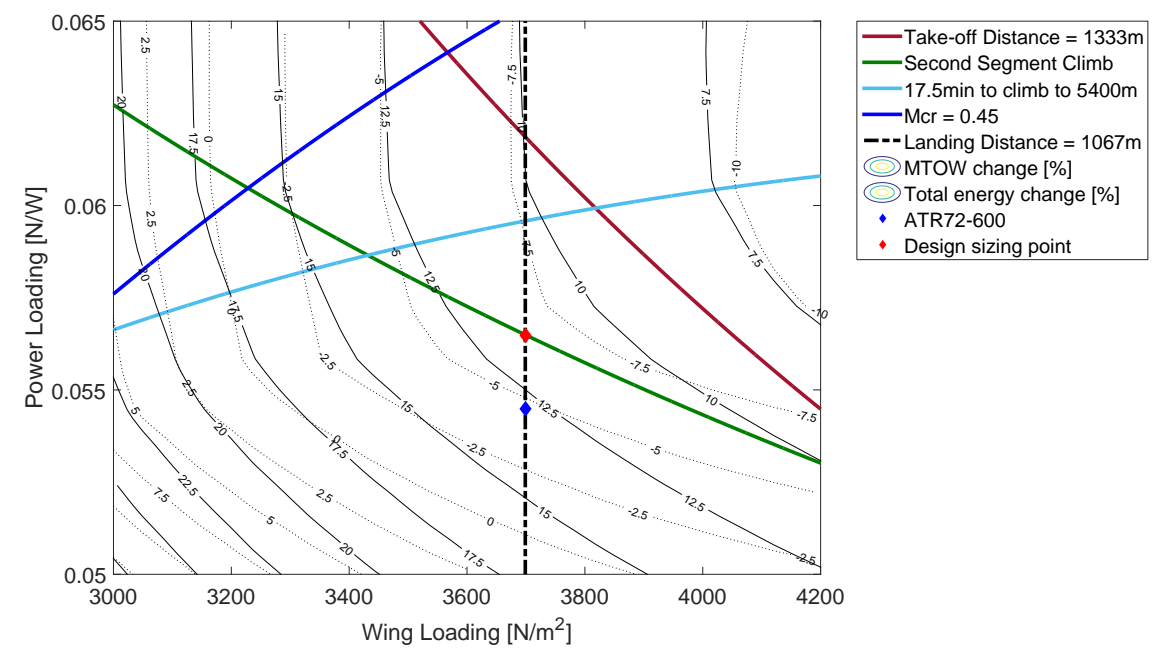

Figure 6.12: Parallel architecture design constraint diagram with iso-mass and iso-energy curves.

With respect to the ATR72 design point, drawn in blue on the diagram, the parallel architecture has the same wing loading but a higher power loading. The wing loading constraint does not change as the propulsive system external architecture is the same in both designs and no change to the maximum lift coefficient are thus assumed. The electrification of the propulsive system has a limited impact on the OEI constraint as it was assumed that in case of engine failure, the motor would not be used to drive the propeller by itself. The resulting power loading is improved by around $1 \%$ with respect to the reference value.

The constraint diagram of figure 6.13 shows the result for the parallel hybrid architecture. Even though the OEI climb gradient constraint are harsher than for the reference aircraft, the active power loading constraint is the take-off requirement as the propulsive power is spread over six units. Thanks to the improvement of the lift induced drag allowed using tip-propellers, the climb constraint is also less severe. In this case there are two optimum points, one for the smallest wing and one for the lightest propulsive system but as they fall extremely close together, the choice between the two does not have a sizeable impact on the overall results.

The tip-propellers help in the reduction of the wing drag but not in providing additional lift as they are relatively small and only part of the flow interacts with the wing upper surface. Therefore, the wing loading constraint is unchanged from the reference study. This design offers an improvement of the power loading of around $9 \%$. The thumbprint curves indicate also that further increasing the power loading would not have a large impact on mass or total energy. This is due to the approach used to in the sizing method where the powertrain is sized by considering requirements derived both from the constraint analysis and from the point mass analysis. For excessively high-power loadings, the mission requirements in terms of maximum climb time or cruise Mach number become sizing for the design and the new power loading overwrites the constraint analysis result.

Lastly, the results for the distributed serial architecture are shown in figure 6.14. Thanks to the higher lift coefficient allowed by the distribution of thrust along the wing span, the wing loading constraint is increased by around $32 \%$ with respect to the ATR72. The improved lift characteristics has also a large impact on the take-off boundary making it less severe. The OEI conditions are also easily satisfied as the loss of one of the PPU decreases the maximum thrust by only $10 \%$.

For this architecture, the sizing point is unambiguous as the optimal power and wing loadings coincide at the intersection of the time-to-climb and landing constraints. The power loading is improved by around 


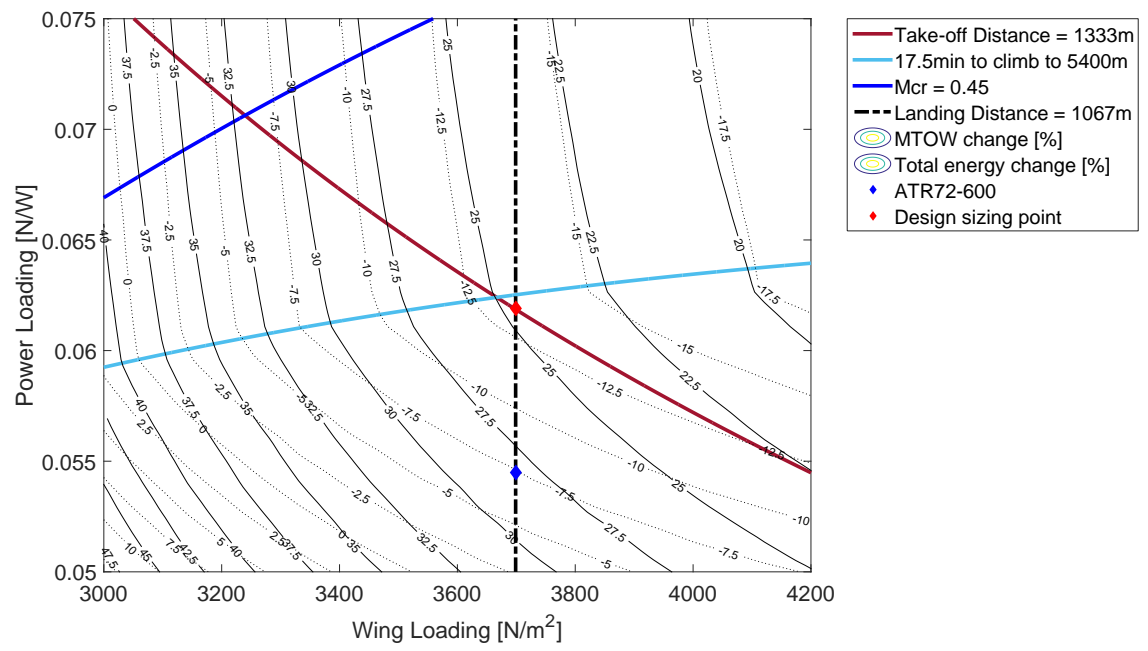

Figure 6.13: Series/parallel architecture design constraint diagram with iso-mass and iso-energy curves.

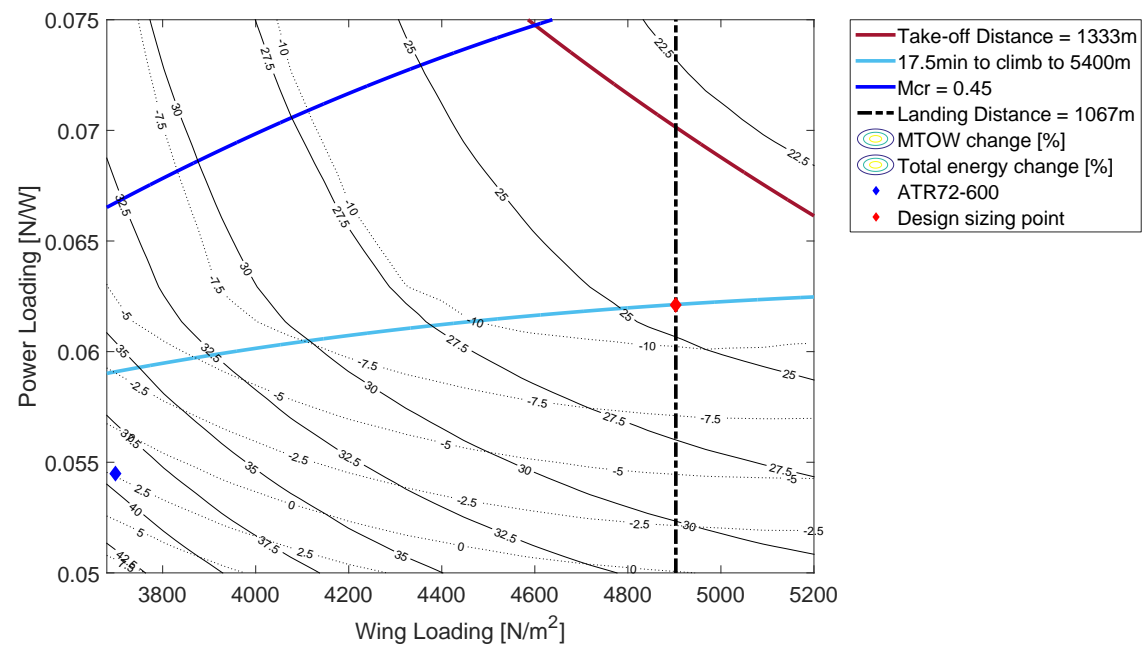

Figure 6.14: Distributed series architecture design constraint diagram with iso-mass and iso-energy curves.

$11 \%$ with this architecture. It can be noticed that if the time-to-climb requirement would be relaxed, a much higher power loading could be attained. As this is not a regulatory requirement, the relaxation would depend on other criteria related to the climb capability of the aircraft and customer desires. However, the constraint is kept unchanged to allow the comparison of the designs in terms of propulsion architecture only.

\subsubsection{POINT MASS ANALYSIS}

In this section the point mass analysis module results are investigated. Attention is given to the power usage along the mission on the propulsive (propeller, gearbox, gas-turbine and motors) and system (battery and generator) sides.

\section{PARALLEL ARCHItecture}

Figure 6.15 shows the power usage of the parallel architecture during the harmonic range mission including the $180 \mathrm{~km}$ diversion. The total propulsive power is drawn in blue, the shaft power, obtained by correcting the propulsive power with the propeller efficiency, is denoted by a black line and the total electric motor and gas-turbine power are in green and red respectively. While propulsive and gearbox shaft power refer 
to the in-flight condition, the electric motor and turbo-shaft are corrected with their respective power lapse coefficients to show the sizing requirements for these machines.
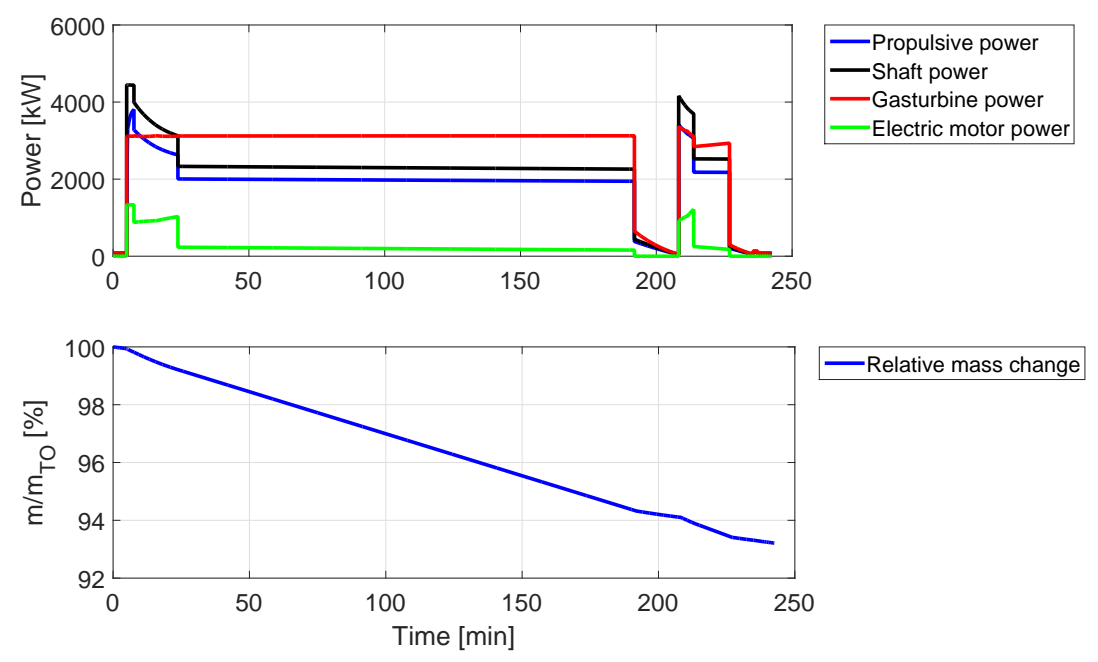

Figure 6.15: Power usage and mass change along mission for parallel HEP design.

In figure 6.15 it can be clearly noticed how the use of electric motors to supply excess power allows the operation of the air-breathing engines at constant power setting. Moreover, it can be observed that, although the propulsive power diminishes with altitude during the climb segment, the electric motor must supply slightly more power to the propeller as the turbo-shaft engine performance degrades with altitude due to the power lapse effect. Overall, the optimal control for this design leads to a low degree of hybridization with the electric motor used mostly during climb and take-off. In the figure below, the change in aircraft mass as the mission proceeds is shown. This change in mass is entirely due to fuel consumption as closed cycle Li-air batteries are used for this design and no change in battery mass happens due to its discharge. The relative zero-fuel weight of this design is around 93\%, two points higher than the reference aircraft as less fuel is stored on-board while the fixed mass is increased by the battery.

Alongside the power usage, it is also interesting to investigate how the efficiency of the propulsive system changes as altitude, speed and power requirements change. In figure 6.16 the electric motor map of one of the two motors installed in the parallel architecture is shown. Iso-efficiency lines are drawn in black and iso-power lines, normalized by the maximum power rating of the motor, are drawn in blue.

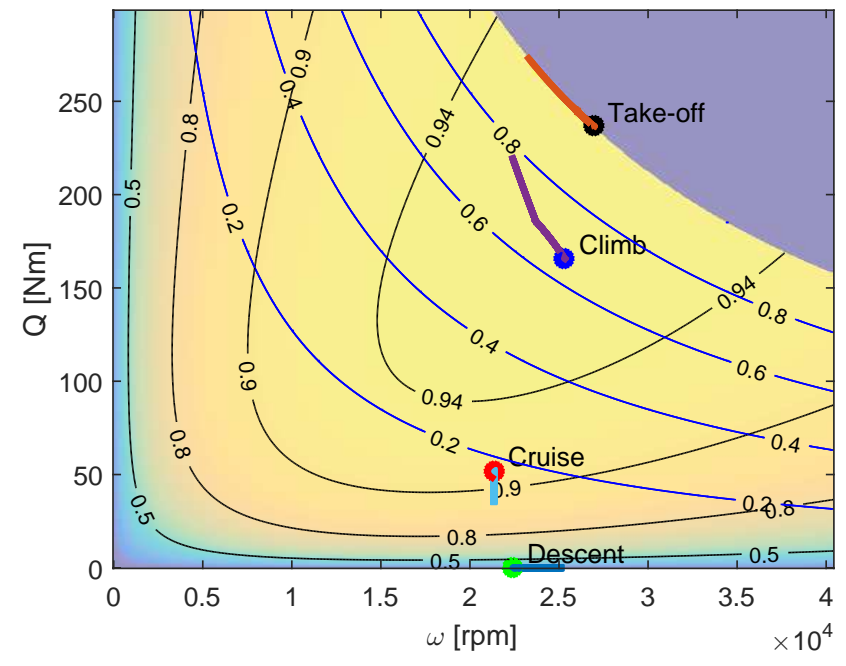

Figure 6.16: Electric motor map with operational requirement overlay for parallel HEP. 
For the parallel architecture, the motor is assumed as mechanically connected on the turbo-shaft power turbine. Definitive numbers for the turbine shaft speed could not be found so a value of $25000 \mathrm{rpm}$ was assumed for this engine that in combination with a gearbox ratio of 22.5 can drive the propeller at the same tip Mach number as the reference design $\left(M_{t i p, \max }=0.76\right)$. As the Mach number changes with altitude and speed, the propeller shaft speed is regulated accordingly so to not exceed the operational constraint. For the resulting $4.13 \mathrm{~m}$ propeller of the parallel design, changes in propeller rotational speed $10-15 \%$ lower than the reference value at take-off conditions are seen. This is in accordance with values found during the validation of the ATR72 propeller as reported in [90].

The mechanical gearbox couples the engine/motor system to the propeller so to avoid over-speed issues, the motor shaft speed is changed accordingly. Since the electric motor shaft power is computed from the performance requirement, the torque can be then derived and used to compute the electric motor efficiency using the method described in chapter 4.2.4. Segments that indicate the change in shaft speed, torque, power and efficiency for each flight condition are overlain on top of the map. On-ground and diversion segments have been omitted to facilitate the reading of the graph. The resulting graph shows that the take-off and climb segments are characterized by the highest motor efficiency while the low power requirement $(20 \%$ of the rating power) of the cruise condition decreases the motor efficiency by 5 percentage points from around $95 \%$ to $90 \%$. Even with this change, this result shows that the electric machines remain highly efficient over a wide range of power outputs. The descent is carried out with no electrification in this case, so the motor efficiency remains at $0 \%$.

Figures $6.17 \mathrm{a}$ and $6.17 \mathrm{~b}$ show the power requirements at the energy sources and the battery discharge characteristics as function of the mission time and of the degree of discharge. The motors in the parallel HEP are completely powered by the battery so no electric generator is present in this architecture.

The first graph shows that large fluctuations in the battery power happens as the mission proceeds as the electric motors need to supply the power necessary to keep the engines at constant operation point. This change is clearly represented in the State Of Charge graph where the high-power requirements compound with a lower efficiency to discharge the battery faster. The take-off and climb condition contribute to $25 \%$ of the battery discharge while accounting for only a $10 \%$ of the total mission time.

The battery is sized with a $20 \%$ SOC limit to avoid damages. This limit is added on top of the diversion energy requirements leading to around $10 \%$ of battery energy that would not be used in normal operation. This clearly has a detrimental effect on the design due to the low specific energy of the battery as any excess of energy has large impact on the performance of the aircraft as seen in the sensitivity study of chapter 6.4. It could be argued that the SOC limit should be set at the harmonic mission end thus assuming that the battery would be completely drained in case of emergency. The choice of having residual energy at the end of the diversion is justified in this study by recognizing that no regulations yet exist and fully discharging the battery is both less safe and irreparable damage to the energy source would require its substitution.

The discharge characteristic of this battery is shown in figure $6.17 \mathrm{~b}$. Differently from the previous figures, the battery output voltage is not graphed as a function of time but as a function of the degree of discharge. This means that if no energy is extracted from the battery during a segment, it will not be visible on the graph. The system voltage is set at $3 \mathrm{kV}$ leading to a requirement of 738 cells in series while the capacity requirements lead to the necessity of six modules in parallel for a total of 4428 electrochemical cells. As seen above, in this design the electric motors are used mostly in take-off and climb with low power requirements in cruise. This leads to the large jumps in battery voltage highlighting also the fact that the chosen control operation favours more a high-power battery than a highly energetic one. Finally, it is interesting to notice that, even though the battery power diminishes as the aircraft gets lighter during the cruise phase, the voltage of the battery, and consequently its efficiency, gets worse.

\section{SERIES / PARALLEL HYBRID ARCHITECTURE}

The second architecture considered uses a parallel hybrid architecture characterized by a power degree of hybridization of $47 \%$ and an energy DoH of $18 \%, 11 \%$ higher than the previously considered parallel HEP configuration. This higher electrification of the powertrain is visible in figure 6.18 where a power shaving operational approach is used to minimize the fuel-based system mass as in the previous design. As the turboshaft engines are less powerful than in the parallel configuration, the power lapse effect is also reduced and 

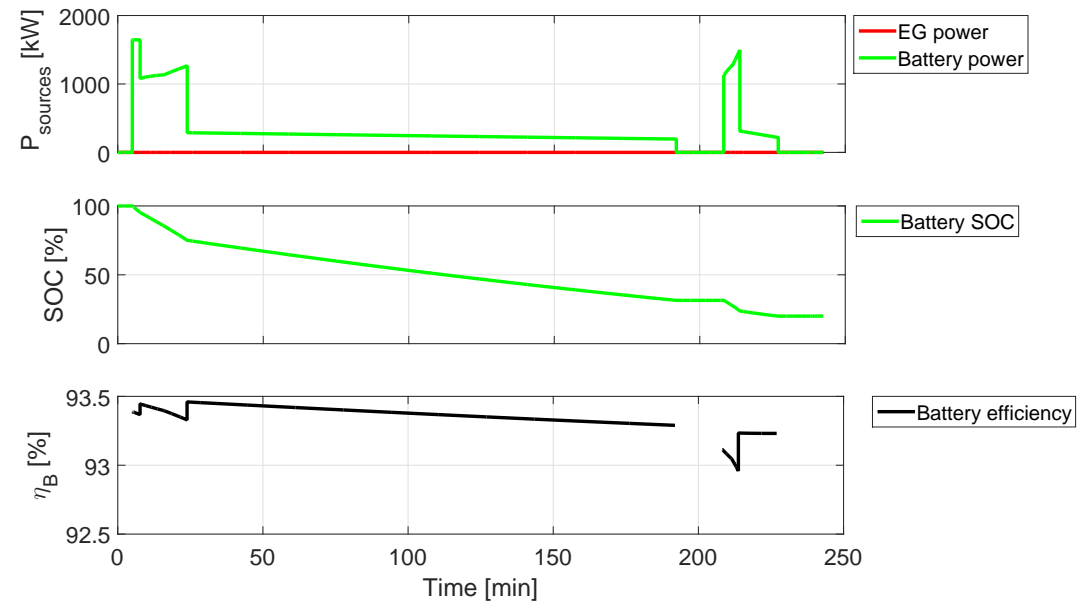

(a) Electric generator and battery power vs mission time.

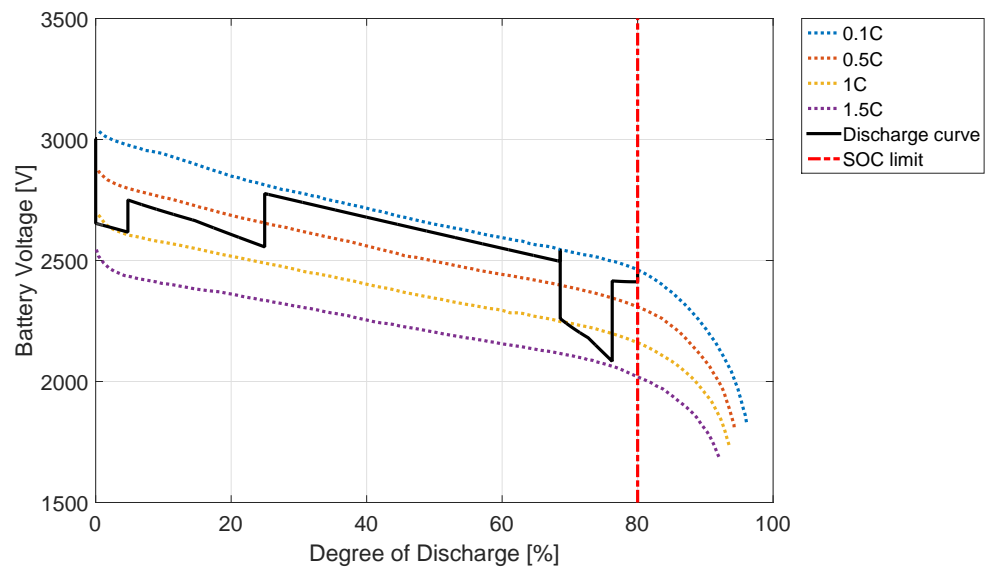

(b) Battery discharge characteristics.

Figure 6.17: System power and battery discharge for parallel HEP.

the requirement variation for the electric motors is less severe. Moreover, the climb segment time is reduced from the reference case by around five minutes possibly thanks to the reduced induced drag.

The aircraft mass change shown in the lower part of figure 6.18 has a similar trend as the one previously discussed but the final relative weight is slightly higher at $95 \%$ of the MTOW. As seen with the higher energy degree of hybridization, less fuel is required to perform the reference mission with respect to its maximum weight.

For this design it is assumed that the electric motor is mechanically connected to the propeller shaft. For this reason, the maximum shaft speed is severely reduced when compared to the parallel architecture results. Therefore, the motor torque needs to be an order of magnitude higher to provide the required power in takeoff and climb as shown in figure 6.19.

Interestingly, the sizing power for this architecture's motors is the top of climb condition and not the take-off phase. As that power is used only for a fraction of the mission, the motors could be down-sized to the take-off requirement and used in an over-powered setting during the climb to optimize the design. When this change was implemented little to no effect was registered as the small reduction in mass was cancelled by a lower motor efficiency, so the change was omitted.

Looking at the motor characteristics during the cruise phase in figure 6.19 it can be noticed that the machine efficiency is higher than in the parallel design case. This is thanks to the smaller utilization gap between climb and cruise conditions that derives from the higher electrification of the cruise segment. Also in this case the 

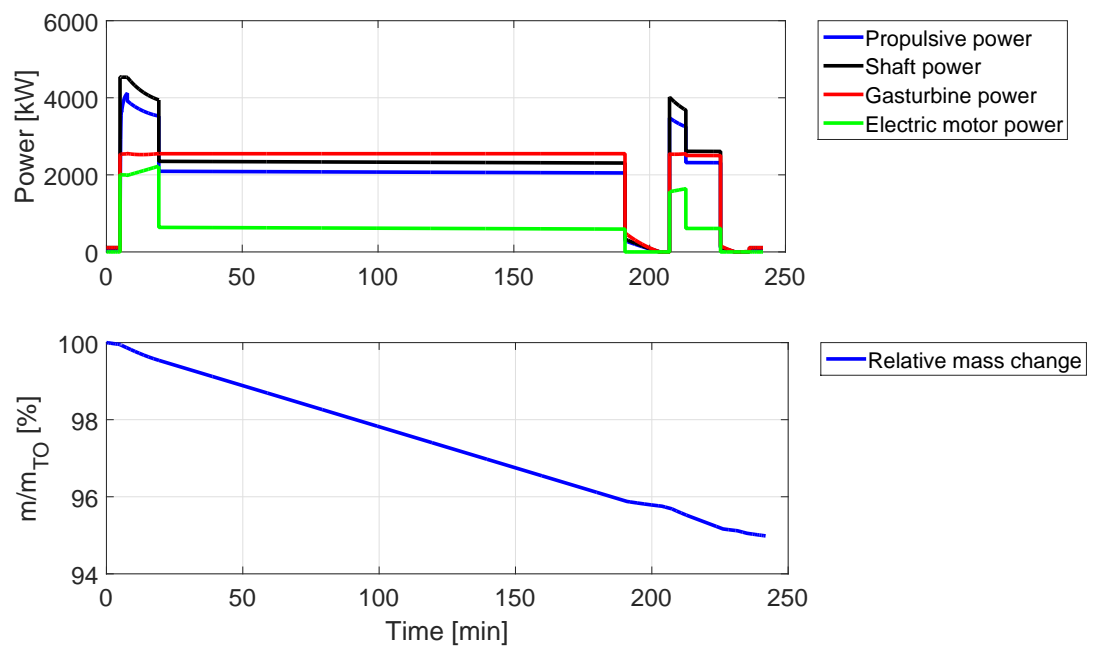

Figure 6.18: Power usage and mass change along mission for series/parallel HEP design.

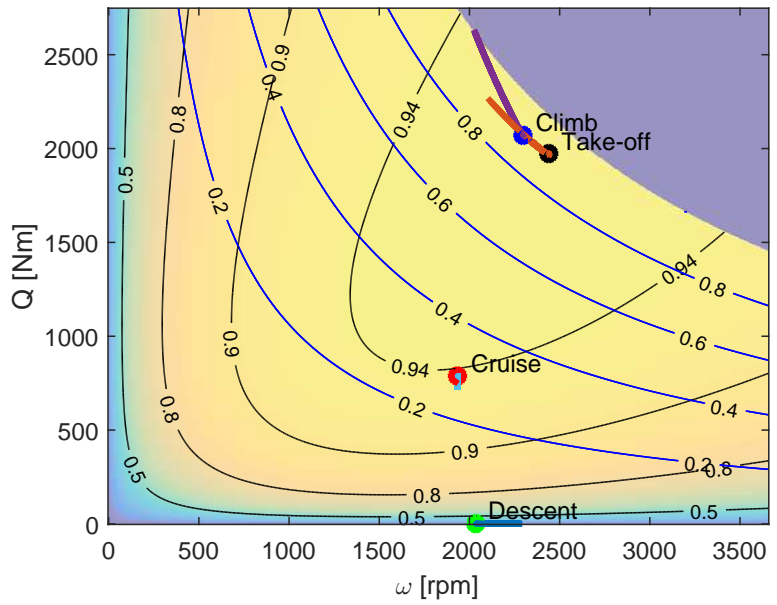

Figure 6.19: Electric motor map with operational requirement overlay for series/parallel HEP.

descent is done without the use of electric power, so the segment efficiency remains null.

Figure 6.20 a shows the battery power utilization, state of charge and efficiency as the mission progress. No generators are used in this architecture as the electric propulsive system is kept completely separated from the fuel-based one. This has potential benefits for the aircraft safety as the electric system can provide around half of the propulsive power required in climb and $100 \%$ of the cruise requirement. The resulting battery for this design has the same number of cells in series as the previously studied design as the system and cell open circuit voltage are kept unchanged. On the other hand, the higher energy requirements lead to the need of nine modules in parallel. The current passing through each cell is equal to the current requirement of the battery divided by the number of modules. Therefore, even though the power requirements are higher, the cell discharge current is decreased by around $30 \%$ with respect to the parallel architecture case. The lower current leads to a slower discharge rate as seen in figure $6.20 \mathrm{~b}$ and higher battery efficiency as shown in the bottom graph of figure 6.20 a.

\section{SERIES ARCHITECTURE}

The distributed series architecture is characterized by a $D o H_{P}=100 \%$ and a similar energy degree of hybridization to the series/parallel architecture at $17 \%$. The peculiar power degree of hybridization is clearly visible in figure 6.21 where the entirety of the propulsive power is produced by the electric motors. A conse- 

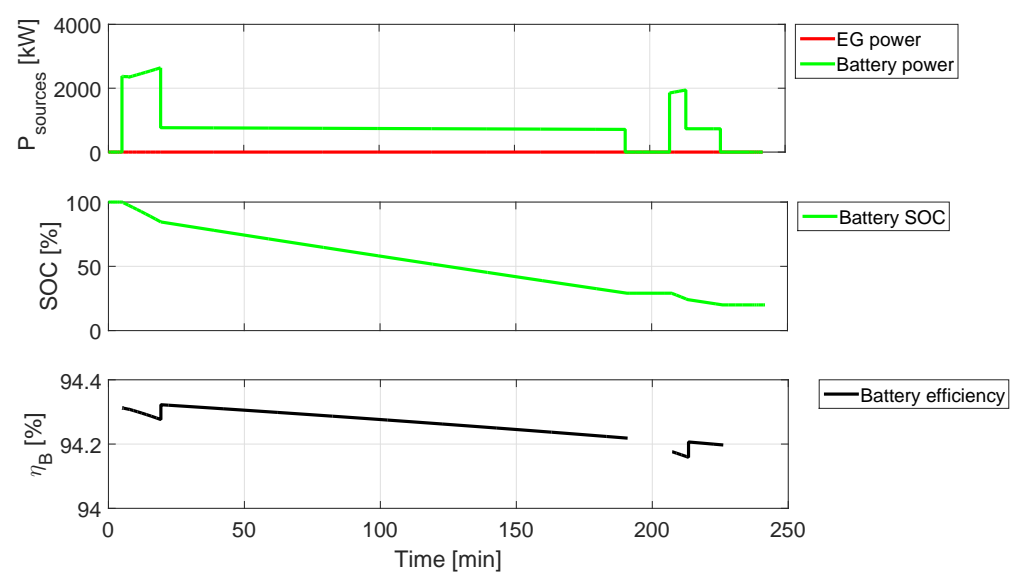

(a) Electric generator and battery power vs mission time.

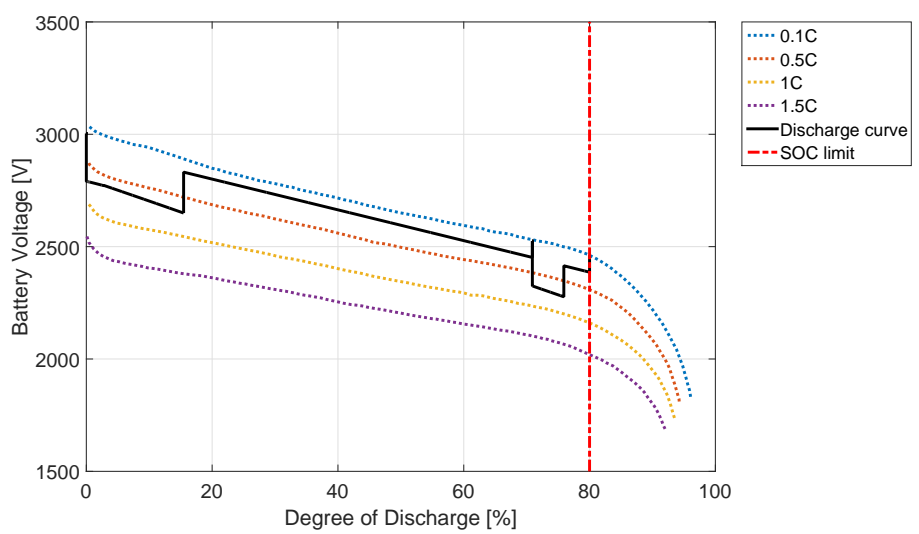

(b) Battery discharge characteristics.

Figure 6.20: System power and battery discharge for series/parallel HEP.

quence of this control strategy is that the propulsive power does not significantly change with altitude thus leading to a faster climb phase. The reduced fuel mass and large battery lead to an end of mission relative mass of 95\%, the same as for the parallel hybrid architecture. This indicates that the zero-fuel weight is more affected by the HEP hybridization than the architecture itself.

The configuration under consideration is characterized by ten propellers of equal size powered by as many motors. It is assumed that the motors' shaft speed is equal to the propeller speed as using a gearbox would only add weight and decrease the system efficiency. The propellers are smaller than in the previous cases as each must provide only part of the propulsive power. However, since the entirety of the propulsion is provided electrically, the motors have sizing requirements comparable to the previous designs (around 500 $\mathrm{kW}$ ). The resulting propeller diameter is half the size of the reference ATR72 propeller at only $1.88 \mathrm{~m}$. The small diameter also means that the propeller can turn faster before its tip reach the maximum allowable Mach number resulting in a nominal rotation speed of $2100 \mathrm{rpm}$, almost double the reference value. This high speed helps in keeping the torque requirement of the electric motor lower as shown in figure 6.22.

Among the motors analysed so far, the results for the series architecture show the best characteristics. The climb and take-off condition are carried out at maximum power rating close to the highest efficiency. Differently from before, also the cruise phase is characterized by the high efficiency since the cruise propulsive power is $100 \%$ electric and the different conditions can be carried out with a smaller power utilization range. The descent segment is the only one that suffers in terms of efficiency as the torque requirements are low. However, since the power output is small, the impact on the energy consumption is negligible.

In the distributed series architecture the thrust is produced entirely electrically, however, the energy is partially sourced from electric generators connected to fuel burning engines as a universally electric aircraft 

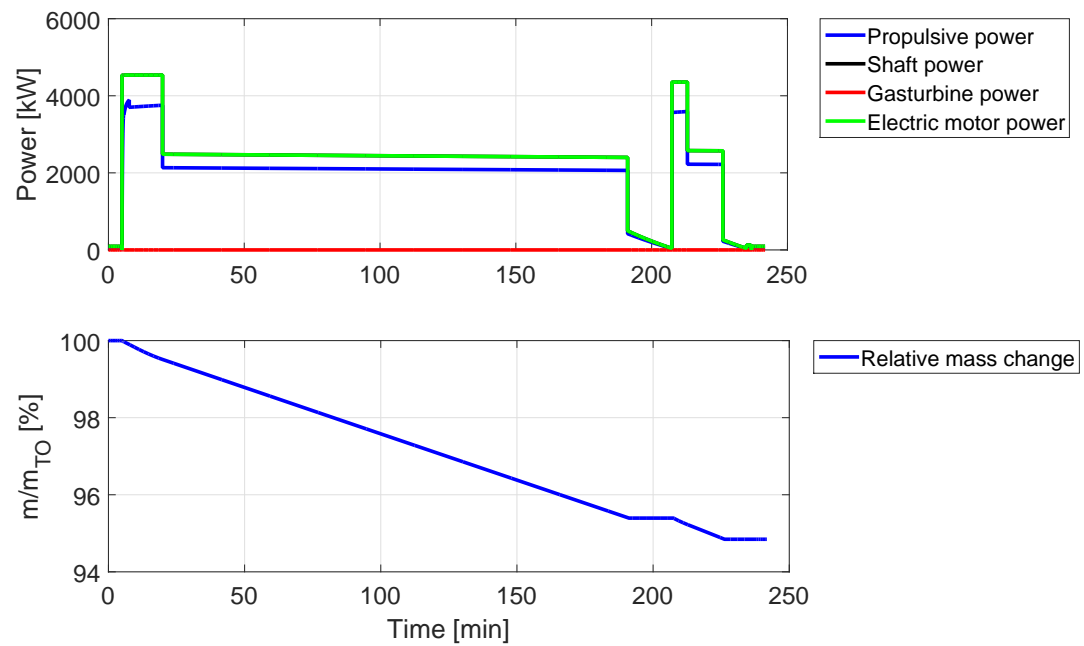

Figure 6.21: Power usage and mass change along mission for series HEP design.

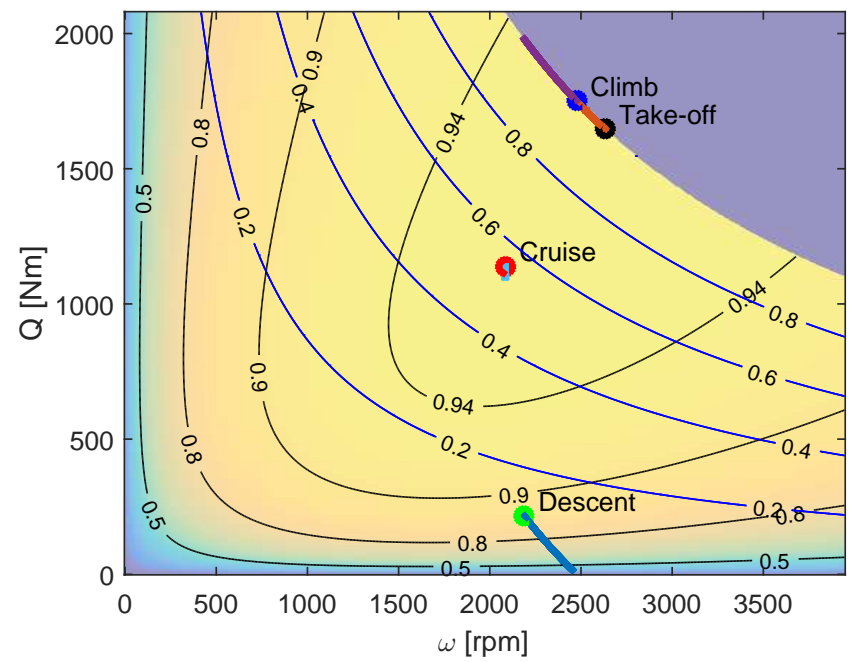

Figure 6.22: Electric motor map with operational requirement overlay for series HEP.

would be too heavy and not competitive in terms of energy consumption as seen in the design space exploration of chapter 6.2. The system gas-turbine (i.e. connected only to the electric generator) and the battery power requirements are shown in figure $6.23 \mathrm{a}$.

As for the previous designs, a power shaving approach is followed in the definition of the control parameters. This results in a constant gas-turbine power and a variable battery output to cover the reduction of turboshaft power due to the reduced air density. Due to the heavier design deriving from the use of a fully electric propulsive system, the requirements at the battery are the largest so far. The number of batteries module required in parallel is 12, double the amount used in the parallel architecture while the number of cells in series remains the same as the system voltage is kept at $3 \mathrm{kV}$ also for this design.

The larger number of modules in parallel has a positive effect on the cell current as seen previously. Moreover, the total resistance of the battery is also decreased as explained in chapter 4.2.7. Both these effects improve the efficiency characteristics of the battery as the Ohmic losses are reduced. This is visible in the bottom graph of figure 6.23 a where the efficiency changes only by a couple of decimal points even when the maximum power is drawn during the diversion climb phase.

Finally, the discharge characteristics of the series architecture are shown in figure $6.23 \mathrm{~b}$. Similarly to the se- 


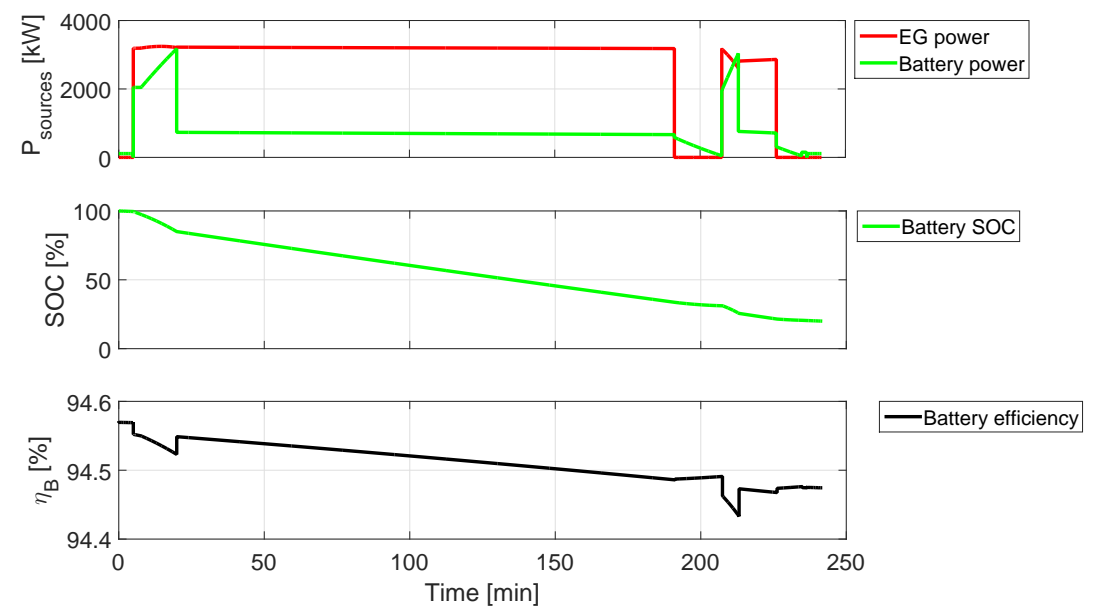

(a) Electric generator and battery power vs mission time.

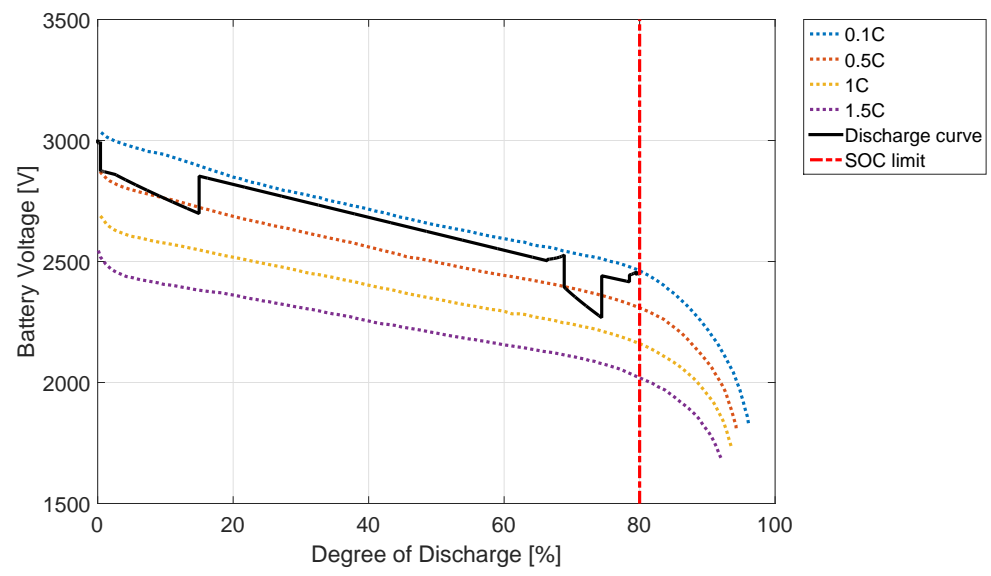

(b) Battery discharge characteristics.

Figure 6.23: System power and battery discharge for series HEP.

ries/parallel hybrid, the low cell current leads to a low discharge rate throughout the mission with the peak during the diversion climb. As the battery is drained, the voltage decreases leading to higher current requirements to supply the power needed for propulsion. As in the previous cases, this leads to a decreasing efficiency. As for the other architectures, the battery still retains $20 \%$ of the capacity at the end of the diversion segments thus leading to an over-sizing of the battery for safety purposes.

\subsubsection{RESULTING DESIGNS COMPARISON}

In this section the three architectures are compared in terms of weights and energy consumption thus finalizing the assessment of the optimized control strategies. In figure 6.24, the mass fractions of the resulting designs can be compared to the baseline aircraft. The MTOW of an aircraft is usually subdivided into fuel, payload and Operative Empty Weight. In the case of a hybrid electric aircraft it makes sense to differentiate between the battery mass and the rest of the OEW even though this mass does not change during the mission and cannot be easily changed unless a modular battery design is used to allow more flexibility in the mission selection. Moreover, since the electrification of the propulsive system has consequences on its mass, the OEW is further subdivided in propulsion group weight (in light blue), wing group (in teal) and other structures (in blue) comprehending everything else that is not directly affected by the hybridization of the propulsive system (fuselage, empennage, landing gear etc.). The payload is the same for all four designs and it is set to 7500 $\mathrm{kg}$ as indicated in the literature for the ATR72 at full-load. 
ATR72 MTOM = 23000kg

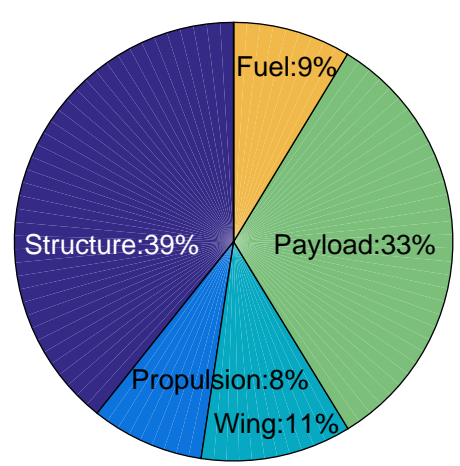

Hybrid MTOM $=28523 \mathrm{~kg}$

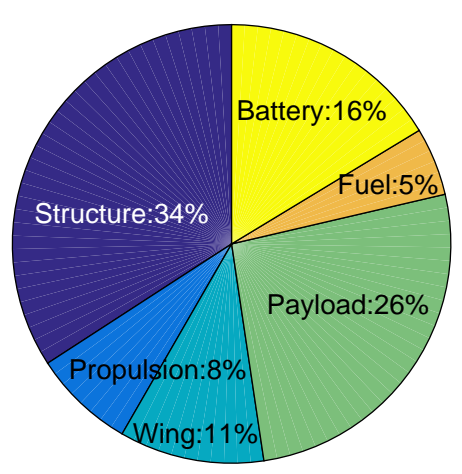

Parallel MTOM = 25614kg

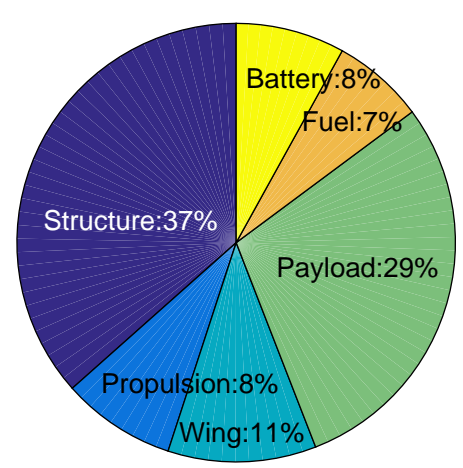

Series MTOM $=28756 \mathrm{~kg}$

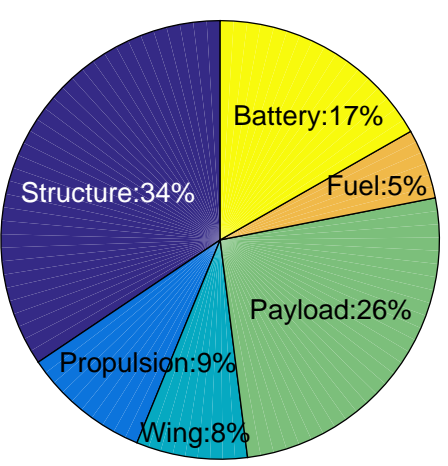

Figure 6.24: Resulting designs mass fractions.

All three hybrid design have a higher MTOM than the baseline aircraft as expected from the low specific energy of the second energy source. Looking at the mass fractions of figure 6.24 , it can be noticed how the battery mass has a predominant place in hybrid electric designs. For example, even though the parallel architecture has a low energy degree of hybridization of $6 \%$, the battery mass accounts for $8 \%$ of the MTOM. This result clearly derives from the different specific energy of fuel and batteries. Nevertheless, as the electrification increases, the fuel mass fraction is reduced. As seen in the design space exploration, this happens only when the correct HEP control laws are used to avoid over-sizing both the fuel-based and electric propulsive systems.

For the first three aircraft, the propulsive group fraction remains constant indicating that even though many components are added in parallel to the fuel-based system, the lower weight of the conventional system balances out the electrical one. This is particularly true for the technology level assumption studied in this chapter as the motors and engine specific weights are comparable and the increase of weight due to the power management and distribution system is not excessive. It is expected that for less optimistic technology maturity assumptions, the propulsion group would make up a larger share of the total.

The series architecture is the only outlier as the share of the propulsion group is higher than in the other cases. This is to be expected as the large number of propulsive units and electric components drives up the total mass of the aircraft. This increase is however balanced out by the lighter wing deriving from this design high lift capabilities that result in a 33\% higher wing loading when compared to the baseline.

Overall, it can be seen how the electrification of the propulsive system allows reductions of fuel consumption with the trade-off of a smaller payload percentage for increasing hybridization ratios. Further studies should 
be conducted in investigating if these new designs can become competitive as even if the fuel load is diminished, the cost of maintaining two systems, the cost of the electricity and batteries, and the higher mass with consequent higher operational costs could lead to un-economically results.

Table 6.2 summarizes the important parameters of the resulting designs in terms of mass, energy requirement and other key performance indicators. The relative change with respect to the baseline design presented in chapter 5 are also shown to better understand the implications of using a hybrid electric powertrain.

\begin{tabular}{|c|c|c|c|c|c|c|c|c|}
\hline \multirow{2}{*}{$\begin{array}{l}\text { Configuration } \\
\text { Power to mass }\end{array}$} & \multirow[b]{2}{*}{$\mathrm{kW} / \mathrm{kg}$} & \multirow{2}{*}{$\begin{array}{c}\text { Baseline } \\
0.176\end{array}$} & \multicolumn{2}{|c|}{ Parallel } & \multicolumn{2}{|c|}{ Hybrid } & \multicolumn{2}{|c|}{ Series } \\
\hline & & & 0.174 & $-1.1 \%$ & 0.168 & $-4.6 \%$ & 0.158 & $-10.2 \%$ \\
\hline Wing loading & $\mathrm{kg} / \mathrm{m}^{2}$ & 377 & 377 & $+0.0 \%$ & 377 & $+0.0 \%$ & 500 & $+32.6 \%$ \\
\hline \multicolumn{9}{|l|}{ Wing } \\
\hline Area & $m^{2}$ & 61.0 & 67.9 & $+11.3 \%$ & 75.7 & $+24.1 \%$ & 57.5 & $-5.7 \%$ \\
\hline Span & $\mathrm{m}$ & 27.0 & 28.6 & $+5.9 \%$ & 30.1 & $+11.8 \%$ & 26.3 & $-2.6 \%$ \\
\hline \multicolumn{9}{|l|}{ Aerodynamics } \\
\hline$C_{D, 0}$ & - & 0.0307 & 0.0303 & $-1.3 \%$ & 0.0293 & $-4.6 \%$ & 0.0327 & $+6.5 \%$ \\
\hline$C_{L, \max }$ & - & 1.3 & 1.3 & $+0.0 \%$ & 1.3 & $+0.0 \%$ & 1.6 & $+23.1 \%$ \\
\hline$C_{L, \max , T O}$ & - & 2.2 & 2.2 & $+0.0 \%$ & 2.2 & $+0.0 \%$ & 3.1 & $+40.9 \%$ \\
\hline$C_{L, \max , L}$ & - & 3.2 & 3.2 & $+0.0 \%$ & 3.2 & $+0.0 \%$ & 4.2 & $+31.3 \%$ \\
\hline Lift to drag ratio & - & 16.9 & 17.2 & $+1.8 \%$ & 18.8 & $+11.2 \%$ & 17.4 & $+2.9 \%$ \\
\hline \multicolumn{9}{|l|}{ Weights } \\
\hline Max. take-off mass & $\mathrm{kg}$ & 23000 & 25600 & $+11.3 \%$ & 28500 & $+23.9 \%$ & 28800 & $+25.2 \%$ \\
\hline Zero-fuel mass & $\mathrm{kg}$ & 21000 & 21800 & $+\mathbf{3 . 8 \%}$ & 22400 & $+6.7 \%$ & 22400 & $+6.7 \%$ \\
\hline Operative empty mass & $\mathrm{kg}$ & 13500 & 14300 & $+\mathbf{5 . 9 \%}$ & 14900 & $+10.4 \%$ & 15000 & $+11.1 \%$ \\
\hline Max. payload mass & $\mathrm{kg}$ & 7500 & 7500 & $+0.0 \%$ & 7500 & $+0.0 \%$ & 7500 & $+0.0 \%$ \\
\hline Design fuel mass & $\mathrm{kg}$ & 2000 & 1740 & $-13.0 \%$ & 1430 & $-28.5 \%$ & 1490 & $-25.5 \%$ \\
\hline Battery mass & $\mathrm{kg}$ & 0 & 2060 & NA & 4660 & NA & 4820 & NA \\
\hline \multicolumn{9}{|l|}{ Propulsion } \\
\hline PPU number & - & 2 & 2 & NA & 6 & NA & 10 & NA \\
\hline Gas-turbine power & $\mathrm{kW}$ & 4050 & 3130 & $-22.7 \%$ & 2560 & $-36.8 \%$ & 0 & $-100.0 \%$ \\
\hline Motor power & $\mathrm{kW}$ & 0 & 1330 & NA & 2230 & NA & 4540 & NA \\
\hline Generator power & $\mathrm{kW}$ & 0 & 0 & NA & 0 & NA & 3260 & NA \\
\hline Battery power & $\mathrm{kW}$ & 0 & 1650 & NA & 2640 & NA & 3180 & NA \\
\hline $\mathrm{DoH}_{P}$ & $\%$ & 0 & 29.9 & NA & 46.6 & NA & 100 & NA \\
\hline Do $H_{P, \text { Sources }}$ & $\%$ & 0 & 34.5 & NA & 50.8 & NA & 49.4 & NA \\
\hline \multicolumn{9}{|l|}{ Energy } \\
\hline Fuel energy & GJ & 85.6 & 74.5 & $-12.3 \%$ & 61.4 & $-28.3 \%$ & 63.6 & $-25.7 \%$ \\
\hline Battery energy & GJ & 0 & 5.1 & NA & 12.3 & NA & 13.0 & NA \\
\hline Total energy & GJ & 85.6 & 79.6 & $-7.0 \%$ & 73.9 & $-13.7 \%$ & 76.6 & $-10.5 \%$ \\
\hline $\mathrm{DoH}_{E}$ & $\%$ & 0 & 6.4 & NA & 17.0 & NA & 17.0 & NA \\
\hline \multicolumn{9}{|l|}{ Efficiency } \\
\hline Avg. system efficiency & $\%$ & 26.4 & 30.9 & $+17.0 \%$ & 35.2 & $+33.3 \%$ & 40.8 & $+\mathbf{5 4 . 6 \%}$ \\
\hline Avg. ESAR & $\mathrm{m} / \mathrm{MJ}$ & 19.1 & 20.4 & $+6.8 \%$ & 22.2 & $+16.2 \%$ & 25.7 & $+34.6 \%$ \\
\hline \multicolumn{9}{|l|}{ Emissions } \\
\hline Flight emissions & $\mathrm{CO}_{2}$ eq. $\mathrm{kg}$ & 6430 & 5610 & $-12.8 \%$ & 4620 & $-28.2 \%$ & 4800 & $-25.4 \%$ \\
\hline Well-to-Propeller & $\mathrm{CO}_{2}$ eq. $\mathrm{kg}$ & 7650 & 7190 & $-6.0 \%$ & 6770 & $-11.5 \%$ & 7030 & $-8.1 \%$ \\
\hline
\end{tabular}

Table 6.2: Summary and comparison with respect to the baseline design of the three HEP analysed.

From the table it can be seen that the three architectures each excel in one of three areas. The parallel design is the one that is most similar to the baseline having the same number of propulsion units, same wing loading and comparable power loading. This architecture is the least electrified leading to the lowest MTOM increase. However, the low electrification and the lack of aerodynamic improvements make so that this design offers the least improvements in terms of fuel burn and emissions.

The series/parallel hybrid architecture leverage the use of tip propellers to improve the drag characteristics 
thus improving its maximum lift to drag ratio by $11 \%$ when compared to the estimated baseline aerodynamic efficiency. Due to the high level of electrification both in terms of power and energy, the design is heavy, and the wing is the largest of the three design. Nevertheless, the wing span remains less than the airport constraint of $36 \mathrm{~m}$ meaning that this design could still operate from the same airports as the baseline aircraft. The higher propulsion distribution has moved the sizing constraint from the OEI condition to the take-off constraint for this design allowing an increase of the power loading of around $5 \%$ but, due to the higher total weight of the aircraft, the overall installed power is still $18 \%$ larger. The increased electrification allows for a more efficient energy conversion as shown by the improved mission average system efficiency. This improvement in addition to the better aerodynamic characteristics lead to this design being the best in terms of fuel and well-to-propeller emissions reduction with implements of $28 \%$ and $12 \%$ respectively.

The series architecture is the one that allows the largest change in propulsive system configuration both internally and externally. In this study, this characteristic is used as the enabler technology for distributed propulsion. This novel propulsive system integration allows for the use of the propellers as a mean of augmenting lift capability of the aircraft thus allowing for a drastic downsize of the wing area. An increase of $32 \%$ of the wing loading is estimated thanks to a $C_{L, \max }$ in landing of 4.2. Higher values for the high lift coefficients have been found in literature but it was decided to use a more conservative estimation as the complexity of the aero-propulsive interactions, during critical phases such as take-off and landing, increase the uncertainty associated with these choices. Even though this design is the heaviest of the three, the high wing loading leads to a smaller wing when compared to the baseline thus reducing its weight fraction as seen before. However, the higher lift capability does not translate to a better aerodynamic efficiency as it was assumed that the turbulent flow of the propeller slip stream would increase the wing zero-lift drag, in particular its friction drag, as seen in the $6.5 \%$ increase of $C_{D, 0}$. An interesting opportunity allowed by the more homogeneous mass distribution along the span and lower wing size is to increase the wing aspect ratio leading to improvements in aerodynamic efficiency and energy consumption.

Among the three designs, the series architecture shows the highest improvement in average system efficiency and ESAR as up to $50 \%$ of the energy is provided by the efficient battery-motor system as shown in the source power degree of hybridization. However, as seen in the design space exploration, a fuel burning engine is still required to avoid excessive increases in terms of aircraft mass. Overall, this design shows interesting improvements in terms of fuel consumption reduction but due to the higher MTOW and lower aerodynamic efficiency, its benefits falls $3 \%$ short of the series/parallel architecture.

\subsection{SENSITIVITY ANALYSIS}

\begin{tabular}{lcccc}
\hline Parameter & Unit & Reference value & Minimum value & Maximum value \\
\hline Battery specific energy & {$[\mathrm{Wh} / \mathrm{kg}]$} & 750 & 300 & 1200 \\
Battery specific power & {$[\mathrm{kW} / \mathrm{kg}]$} & 0.8 & 0.3 & 1.3 \\
EM/EG/EC & {$[\mathrm{kW} / \mathrm{kg}]$} & $9 / 20 / 15$ & $3.5 / 7.8 / 5.8$ & $15 / 33 / 25$ \\
specific power & {$[\mathrm{V}]$} & 3000 & 500 & 5000 \\
System voltage & 170 & 70 & 270 \\
Propeller disk loading & {$\left[\mathrm{kW} / \mathrm{m}^{2}\right]$} & 1530 & 1200 & 1800 \\
Harmonic range & {$[\mathrm{km}]$} & 5500 & 4500 & 7600 \\
Cruise altitude & {$[\mathrm{m}]$} & &
\end{tabular}

Table 6.3: Parameter variations for sensitivity analysis

Up to this point, the design evaluations have been done with fixed characteristics to allow for the comparison between architectures. In this section, the three proposed HEP configurations and their control strategies are kept unchanged while the sizing values for the main components and characteristics of the propulsive system are varied to assess the sensitivity of the model to these changes. Table 6.3 summarizes the parameters used for this analysis along the upper and lower bound considered. Only one parameter is changed at a time while the other are kept constant at their reference value, also reported in table 6.3. The sensitivity of the designs is studied in terms of MTOM and total energy and the results are reported in figure 6.25 as relative changes with respect to the baseline results. The latter three parameters (propeller disk loading, range and altitude) have an impact also on the baseline design characteristics so the results are normalized with the results found for 
a given value of the parameter. Therefore, the results in the latter three graphs of figure 6.25 show the change with respect to a new baseline design for each value studied.

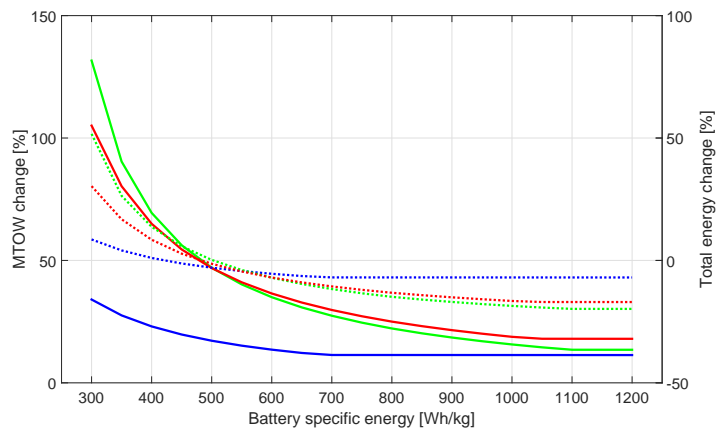

(a) Sensitivity to battery specific energy.

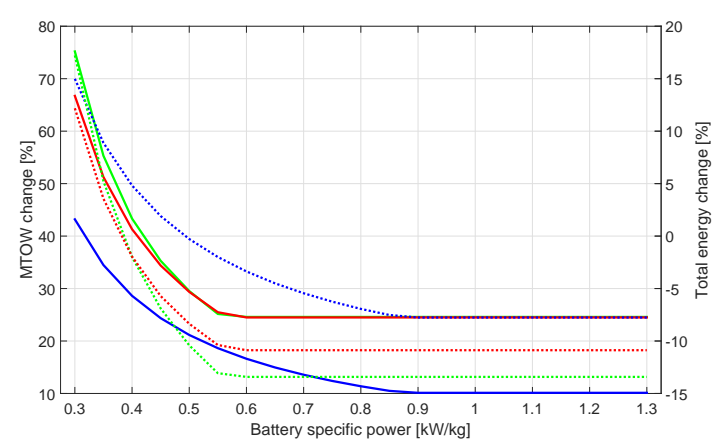

(b) Sensitivity to battery specific power.

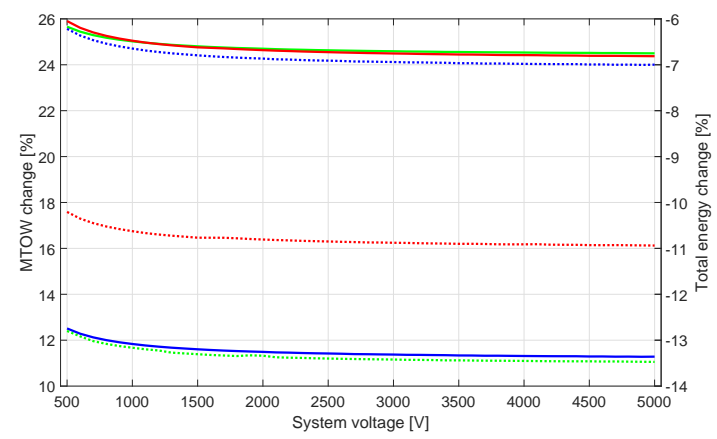

(d) Sensitivity to system voltage.

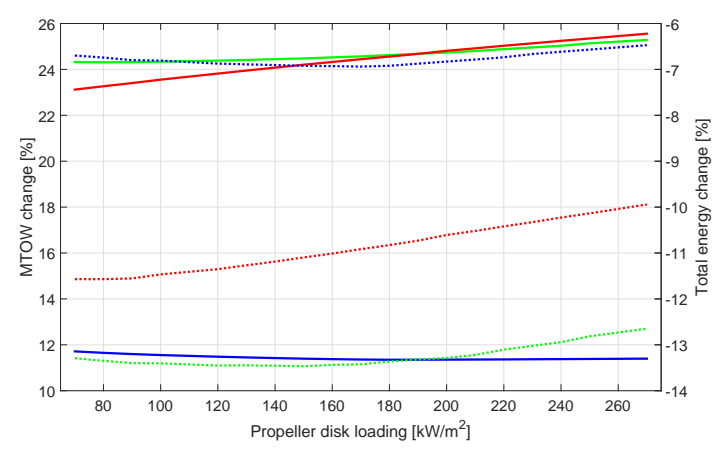

(f) Sensitivity to propeller disk loading
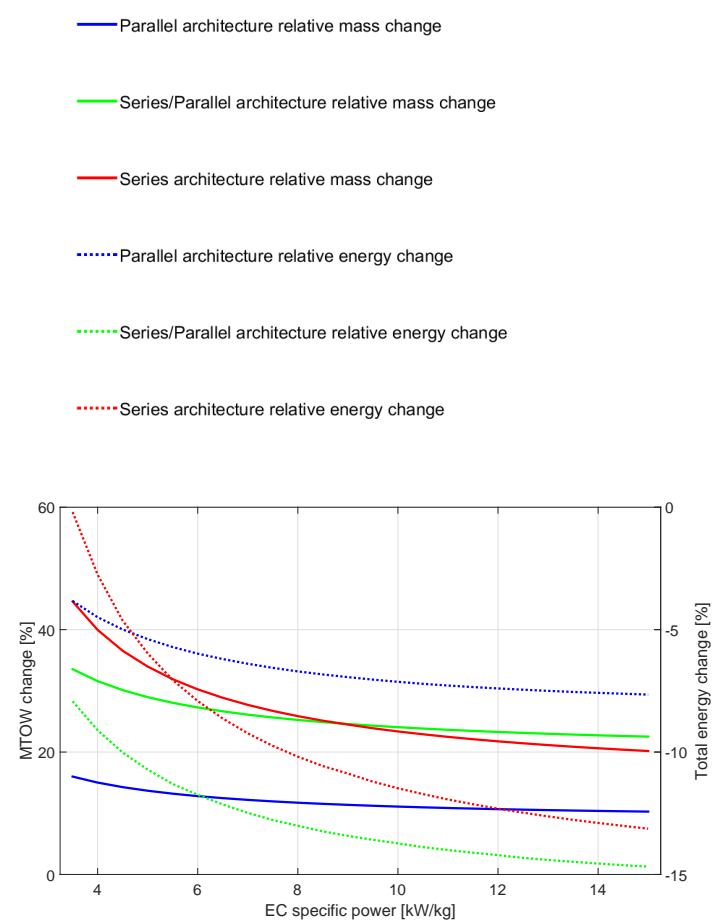

(c) Sensitivity to electric components specific power.

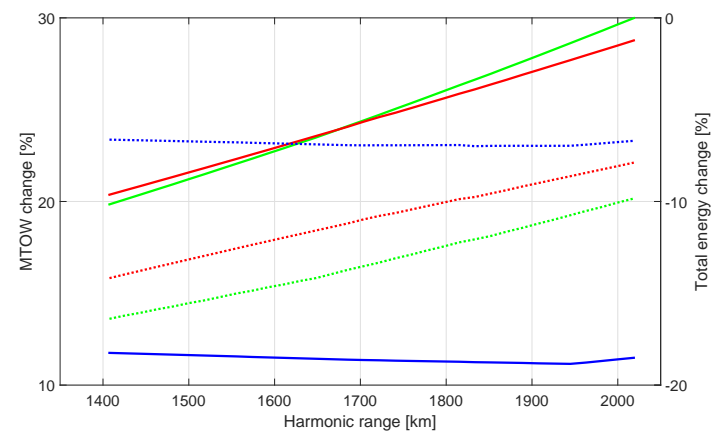

(e) Sensitivity to harmonic range.

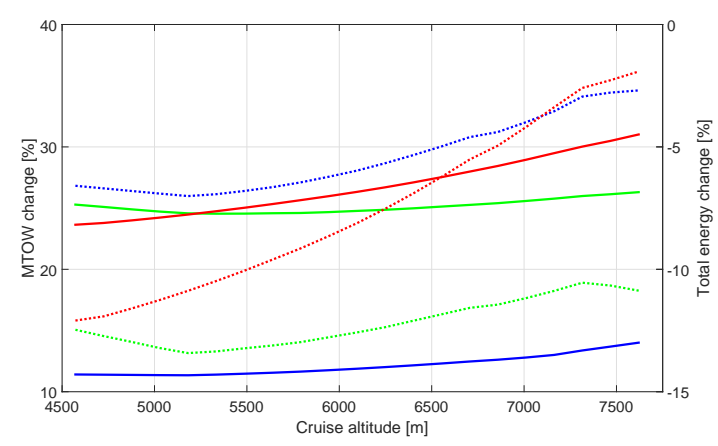

(g) Sensitivity to cruise altitude.

Figure 6.25: Sensitivity study results in terms of MTOW and total energy changes relative to baseline design.

Figure 6.25 a shows the impact of the battery specific energy on the aircraft mass and total energy for the three 
architectures considered. The range varies between 300 and $1200 \mathrm{Wh} / \mathrm{kg}$, where the lowest value could be attained soon while the upper one is theoretically reachable only with highly energetic chemistries such as the open-cycle lithium-air battery. The results show a strong sensitivity to this parameter for all architectures with also a strong correlation to the $\mathrm{DoH}_{E}$. In fact, the hybridization controls assumed for the parallel architecture result in a low electric energy requirement and, as a direct consequence, the lowest sensitivity to the specific energy parameter. Changes of around $25 \%$ for the MTOW and of $10 \%$ for the total energy when comparing the worst and best-case scenarios. It can be also noticed that there is little to no improvement after a specific energy of $700 \mathrm{Wh} / \mathrm{kg}$ as the sizing constraints for the parallel configuration battery changes from the energy to the power requirement. Series/parallel and series configuration show a stronger sensitivity to this parameter with a change of more than $100 \%$ of the mass and around $50 \%$ for the total energy. These two architectures become more energy effective than the parallel one for gravimetric specific energy over $550 \mathrm{Wh} / \mathrm{kg}$ but never match the low mass of the parallel architecture in the range studied.

Similarly, the three architectures show a strong sensitivity to the battery specific power as shown in figure 6.25b. The largest change is seen by the two architectures that are characterized by the higher power sources degree of hybridization (i.e. the battery power over the sum of fuel and battery power) as this implies a higher utilization of the battery during high power segments. The flat-line shown by series-parallel and series architecture's results signifies that after the $550 \mathrm{~W} / \mathrm{h}$ mark, their batteries are sized by the mission energy requirement. On the other hand, the parallel architecture keeps improving until the battery specific power reaches $900 \mathrm{~W} / \mathrm{kg}$ as this design uses the battery mostly during take-off and climb.

The third study aims to capture the sensitivity of the designs to the specific power of the electric components of the propulsive system. A fixed ratio is assumed between the electric motor, generator and PMAD components so that the change of the main variable, the electric motor specific power, is applied to the rest of the parameters. The results are visualized in figure $6.25 \mathrm{c}$. The parallel architecture shows a low sensitivity to these parameters as changes of less than $5 \%$ are seen over the considered range going from 3.5 to $15.5 \mathrm{~kW} / \mathrm{kg}$. The hybrid architecture shows a higher sensitivity but the DEP one is the most affected of the three. This is explained by comparing the three architectures power degree of hybridization. A higher $\mathrm{DoH}_{P}$ means that more of the propulsive power is generated with energy flowing through the electric parts of the hybrid propulsive system. The series architecture surpasses the parallel configuration already for motor specific power of $5 \mathrm{~kW} / \mathrm{kg}$, a value that is already attainable with today' state of the art machines. However, it is important to keep in mind that this result was obtained assuming that the battery would be sized with $750 \mathrm{Wh} / \mathrm{kg}$, a value that will be realistically difficult to attain by 2035 .

The last parameter studied that directly impacts the electric components is the system voltage. The system voltage has two main effects on the studied system. Firstly, a higher voltage means that a smaller current is required to supply the electric power necessary for propulsion. The current has a direct effect on the system efficiency as the Ohmic losses grow with its square. On the other hand, to safely maintain a higher electric potential, the insulator thickness of the cables must be thicker, thus increasing the cable system mass. Moreover, to produce a high battery voltage, many cells must be connected in series. This increases the overall resistance of the battery and its heat losses. The results in figure $6.25 \mathrm{~d}$ show that the aircraft mass and total energy improve as the system voltage is increased thus indicating that the weight and efficiency savings allowed by the lower current outweighs the losses due to higher battery resistance and heavier insulation. In general however, the designs show a low sensitivity to this parameter, so a very high system voltage should be avoided as the increased risk of electric arches is not supported by a comparable improvement in performance.

Figure 6.25e shows the design sensitivity to the sizing mission range normalized by the results of the baseline design as for increasing range the total mass and energy requirements increases for all aircraft considered. The parallel architecture shows that it is not highly affected by the increase of range meaning that its increase of mass and energy have comparable trends as the one seen for the reference design. On the other hand, the other two architectures become relatively heavier and less energy effective as the range increases. This can be explained by the difference in energy and power degree of hybridization. For the parallel architecture, the electric system is mostly used for moving the sizing point of the conventional system from the take-off to the cruise phase, so a longer range does not lead to a large impact as the change in reference values is comparable to the change seen by this design. The other two architecture however use a large amount of electric battery energy during the cruise, so a longer mission leads to worse performances. This study indicates that a low $\mathrm{DoH}_{E}$ design could be used on a wide range of missions while the more electric solutions would have larger 
trade-off between payload and range.

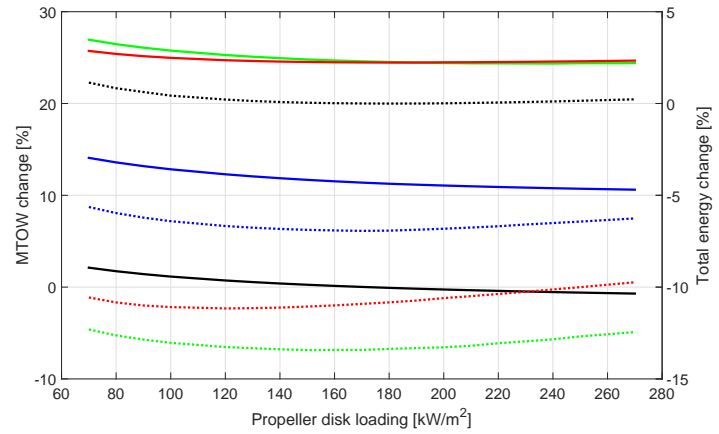

(a) Sensitivity to propeller disk loading.

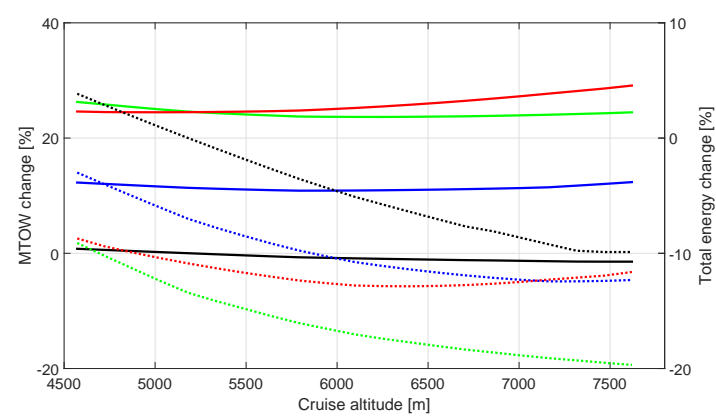

(b) Sensitivity to cruise altitude.

Figure 6.26: Sensitivity study results in terms of MTOW and total energy changes.

In figure $6.25 \mathrm{f}$ the results obtained through the variation of the propeller disk loading are visualized. When this parameter is increased, the propeller can provide a larger power with the same area, or more appropriately for this study, the same propulsive power with a smaller area. One direct consequence is that the smaller propeller will weigh less. However, the higher loading implies that the air mass needs to be accelerated by a larger amount to provide the same level of thrust. This has detrimental effects on the propulsive efficiency and is mirrored in the actuator disk theory where for decreasing propeller diameters the efficiency decreases. These two effects can be seen in figure $6.26 \mathrm{a}$ where the results are normalized by the sizing point of each architecture as the MTOW diminishes for each configuration considered as the disk loading increases. Looking at the total energy, an optimum exists for each configuration. For the reference, parallel and hybrid configurations the reference loading of $170 \mathrm{~kW} / \mathrm{m}^{2}$ is already optimal while for the series one, a lower disk loading is suggested for improved energy consumption. This is because the DEP configuration has already the smallest propellers, so the increase of efficiency given by the smaller disk loading is not nullified by the higher mass.

Figures $6.25 \mathrm{~g}$ and $6.26 \mathrm{~b}$ show the results for the sensitivity to the cruise altitude. In the first figure the results are normalized by the reference design values while in the second they are normalized by the sizing point of each configuration. The change in altitude has little effect on the overall mass of the aircraft as a small amount of additional fuel and electric energy are required to climb to the new altitudes. However, a larger impact is seen for the total energy where the higher altitude leads to better results in terms of energy consumption. This can be explained by the lower air resistance that needs to be overcome as the air density decreases with altitude. When looking at the normalized results with respect to the baseline, the energy improvement is larger for the conventional design meaning that a decreasing return in terms of energy benefits is attained by flying these designs at higher heights. The results of this last analysis are more complex to explain as the cruise altitude has direct effect on the constrain analysis, conventional engines sizing and aerodynamic proprieties of the aircraft. Therefore it is suggested that further investigations on the topic of optimal mission profile is conducted for hybrid electric aircraft. 



\section{CONCLUSIONS \& RECOMMENDATION}

\subsection{CONCLUSIONS}

A method for the conceptual design of a generic hybrid electric powertrain has been proposed in this thesis to enable the study of novel aircraft configurations accounting for new challenges posed by the co-existence of multiple energy sources. The proposed method is based on a basic building unit, the propulsion power unit, that encloses the main components necessary to model a hybrid propulsive system. With the use of few control parameters such as the number of propulsive units, the propulsive power share, the shaft power ratio and the electric power ratio, these units can be combined and modelled into any of the most studied propulsive system architectures.

The control parameters can be supplied by segment specific control laws so that not only the impact of the HEP architecture but also of the control strategy can be assessed and optimized for a series of constraints and mission requirements. The proposed method integrates the main conceptual design procedures, the constraint analysis, the mission evaluation and the system sizing, into an integrated framework that allows the sharing of information between these modules. This ensures coherence in the different modules results. It was found that the electrification of the propulsive system and novel architectures impact the power loading requirements of the designs in a beneficial way. The main drivers for these changes are the opportunity to spread the requirement over many propulsive units and improved power-lapse characteristics.

The main devices of the hybrid propulsive system are sized using mass specific parameters that depend on the technology maturity level expectations. In this way, the components are "rubberised", and they can be easily scaled to the required power rating. The lower fidelity of this approach is a trade-off that enables a quick design space exploration and simplifies the use of these components in vastly diverse propulsive system architectures. The performance characteristics of these devices are modelled with a higher level of fidelity. The energy conversion and power transmission efficiencies change depending on the power requirement, the aircraft state throughout the mission and the component specific performance parameters.

The proposed method is first used in a design space exploration exercise to evaluate the impact of propulsive system architectures, technology levels and control strategies on few key performance indicators. For the most conservative technology assumption, the more conventional architectures studied, parallel and series/parallel, show little to no improvements in terms of total energy and fuel consumption with around $5 \%$ improvements possible only for the optimal control strategy. The more electrified architecture, the distributed series configuration, is characterized by a large increase of mass and energy consumption. The best control strategy for this design still leads to an increase of $40 \%$ of the energy requirements.

For more optimistic technology assumptions, the parallel and series designs are characterized by fuel mass reductions in the order of 10 and $20 \%$ with corresponding total energy savings of 6 and $11 \%$. The hybrid architecture shows better results thanks to the improved aerodynamic efficiency and relatively smaller mass increase when compared to the series architecture. Fuel mass reductions of $26 \%$ corresponding to a total energy saving of $12 \%$ can be realised with a $50 \%$ level of hybridization in terms of power and a $20 \%$ electrification of the energy sources. 
For futuristic technology assumptions, considering high temperature superconductors devices as ready for use in commercial applications, the energy requirements are further improved. The optimal control strategy for the parallel architecture suggests a $50 \%$ hybridization of power and energy leading to fuel savings in the range of $30-40 \%$ over the baseline reference. The design space exploration indicates that the universally electric approach becomes the most effective for the series architecture. The high efficiency and power density of the super-conductive electric system lead to total energy reductions in the range of $30-35 \%$ with a $100 \%$ reduction in fuel load and zero-emissions in-flight. Similarly, the series/parallel configuration optimal control strategy indicates that the fully electric option leads to the largest improvements in terms of total energy and ESAR thus making this configuration a de-facto series architecture. This design does not achieve the same results as the distributed series one since it is encumbered by a heavier wing and reduced overall efficiency due to the low level of power distribution and consequent high electric current related losses.

Changing the control laws from constant for each segment to linearly changing allows for a more "fine-tuned" control strategy that leads to improvements in terms of mass and energy consumption as the over-sizing of propulsive devices is (partially) avoided. Using an optimizer with the HEP control parameters as the design vector could lead to the optimization of the control strategy with respect to the mission requirements and HEP architecture chosen. Due to the lack of this option, a power shaving approach has been chosen as an interesting option for the HEP configurations considered. This control strategy has the advantage of sizing the conventional fuel-based propulsive system for the cruise condition so that it can be run at constant power and maximum efficiency for most of the mission. With this strategy, the configurations MTOM is reduced by around $3 \%$ and the total energy by $5 \%$ with respect to the starting points selected during the design space exploration.

The constraint analysis of the three architectures indicates that propulsive units number, segment specific electrification and changes to aerodynamic characteristics have a large impact on the power and wing loading. The number of PPUs makes the OEI constraints less severe while it does not influence the AEO requirements. The electrification of the propulsive system has a beneficial impact on requirements that need to be satisfied at high altitude such as the cruise Mach and service ceiling requirements. The reduction of the drag coefficient lessens the severity of all the power loading constraints while the higher lift coefficient resulting from the assumption of propulsion enhanced lift leads to a smaller wing requirement. All three designs considered see improvements in power loading with the most conventional architecture, the twin engine parallel HEP, seeing the smallest benefits at $1.1 \%$ reduction and the DEP design seeing the largest at $10 \%$. Parallel and series/parallel hybrid are characterized by the same wing loading as the reference design while the series configuration sees a $33 \%$ reduction in wing loading leading to a $6 \%$ area reduction with respect to the baseline. All three designs have wing span less than the $36 \mathrm{~m}$ constraint meaning that they can be used in the same airports as the reference design.

The comparison of the designs' mass fractions shows that using hybrid electric powertrain based on battery technologies as energy source leads to a smaller paying mass fraction. Moreover, the costs related to the MTOW will be higher for this type of aircraft. However, it is possible that the lower cost of electricity and the lower fuel consumption could lead to profitable designs. This analysis is not developed in this study, but it could be an interesting future development of the work. The parallel and series/parallel configurations maintain propulsion group and wing mass fractions like the reference aircraft while the series one is characterized by a smaller wing but heavier propulsion group. The many components and fully sized electric and fuel-based systems (i.e. two engines along their fuel system and tanks are still required even if the $D o H_{P}$ is equal to 1) make this design the heaviest of the three studied with a $25 \%$ increase in MTOM.

A sensitivity analysis is used to assess the impact of the main performance parameters assumptions. The results show that the most critical obstacles in making HEP designs feasible are the battery sizing characteristics. If battery technology does not reach at least $500 \mathrm{Wh} / \mathrm{kg}$ and $0.5 \mathrm{~kW} / \mathrm{kg}$ capabilities, it seems that no benefits can be obtained from the electrification of the propulsion system. These results are in line with results found in literature. Depending on the level of hybridization, a large sensitivity to the specific power of the propulsive units is also observed. Fully electric architectures such as the series one are the configurations that are most negatively impacted if the motor and battery performances do not improve from today's state of the art technology, while the parallel architecture seems a safer option in terms of technology uncertainty as it is less affected by these parameters. The resulting designs do not show a high sensitivity to the distribution voltage if at least $1 \mathrm{kV}$ is used to provide the required power for the propulsion system. Below that, the current related losses and weight increase of the cabling system impact negatively the aircraft performances 
so developments in high-power cables for aircraft system is seen as another critical technology.

When comparing the key performance indicators, the series/parallel and series designs show similar results in terms of fuel load and in-flight emissions reductions of 29 and $26 \%$ respectively, with the former being the best by 3\% thanks to the lower MTOW and higher lift-to-drag ratio. The parallel architecture is characterized by more modest results with a fuel reduction of around $13 \%$ as the low level of hybridization does not lead to a large improvement of the propulsive system efficiency. Although large, these improvements fall short of the 2035 objective of a $60 \%$ reduction in $\mathrm{CO}_{2}$ emissions wanted by governmental and international agencies. However, these changes to the propulsive system would not be implemented alone in a future design but they would complement other innovations in aircraft design such as lighter structures, better air-breathing engines, greener fuels and possibly better aerodynamic characteristics. It seems then possible that the objective could be accomplished in the considered time-frame.

From these results, it is concluded that the series/hybrid configuration is the most promising in reducing fuel consumption and emissions as it is also a simpler configuration when compared to the DEP architecture and its complex control requirements that couple lift production to the propulsive power. If reality does not match the performance expectations of the electric components, a low hybridization architecture using parallel configuration is instead seen as the only feasible option due to its lower sensitivity to electric components technology advancement.

\subsection{LIMITATIONS AND RECOMMENDATION}

The presented method allows for the conceptual design of a large variety of hybrid propulsive systems with an acceptable level of detail. However, a series of assumptions have been made to simplify the model when the work would have exceeded the scope of the project. These simplifications can be worked upon if a higher level of detail is required:

- Detailed turbo-shaft engine performance model: the fuel flow computation has been handled with the use of fuel maps. These maps are normalized with the reference shaft power allowing their use for any power rating resulting from the point mass analysis. However, engine efficiency changes with its size so these maps would realistically lose validity when straying away from the baseline reference engine. A future development of this project could implement a model that accounts for these differences, so the fuel computation uncertainty can be reduced.

- Detailed propeller model: on a similar note, the propeller is modelled with a low fidelity method. The actuator disk theory is not suitable for estimating high power, low advance ratio and high advance ratio conditions as the complex losses that happen in these conditions are not modelled. If the method capability is to be improved from a purely conceptual to a preliminary level, the use of a higher order method for the propeller efficiency estimation is advisable. A good solution is offered by the Blade Element Method that scarifies part of the scalability and adaptability of the ADT approach for a more refined estimation based on the geometry of the propeller blades (e.g. element lift polars, twist distribution and pitch) and propeller operational characteristics (e.g. shaft speed, flight velocity).

- Heat management for electric components: the sizing parameters used in the sizing of the electric components considers also the cooling equipment requirements. However, the amount of heat produced might require novel approaches efficiency dissipation and control. This could be particularly problematic for the cabling and battery systems as these components could be more integrated into the structure and have less access to fresh air when compared to the motors. Therefore, further research could be conducted in assessing if cooling requirements are critical for HEP architectures.

- Volumetric requirements of HEP systems: The volumetric requirements have been omitted from this study, but it is possible that for high degree of energy hybridization, the space to store the battery packs could be insufficient. Moreover, when geometry is taken into consideration, it could be interesting to assess the optimal placement of a battery in the aircraft airframe. Trade-off between an easily accessible and swappable battery or a highly integrated one that allows weight savings (e.g. by incorporating it into the wing structure as a mean of lift load alleviation) could be performed as part of this study.

- In-depth failure analysis of propulsive system: OEI constraints have been considered in this study and the mitigation of a generator/battery pack failure can be modelled with the use of parameters that in- 
dicate a required redundancy and over-sizing deemed safe. However, differently from fuel distribution system, an electric system can be reconfigured with the use of high-power breaks to change the capacity and power capability of the system. This capability could offer less demanding failure modes so an investigation into this argument could lead to further improvement of the HEP designs.

- Aero-propulsive interaction model: in this study, assumptions on the possible improvements that can be obtained with the use of tip-propellers and distributed propulsion have been modelled with simple segment specific aerodynamic coefficients corrections. A higher order method to simulate these interactions that can still be applied in a conceptual design environment would improve the validity of the sizing process and possibly lead to more insights on the optimal combination of external and internal HEP configurations and hybridization control strategy. 


\section{BIBLIOGRAPHY}

[1] K. R. Antcliff and F. M. Capristan, Conceptual Design of the Parallel Electric-Gas Architecture with Synergistic Utilization Scheme (PEGASUS) Concept, in 18th AIAA/ISSMO Multidisciplinary Analysis and Optimization Con (2017).

[2] P. Busquin, P. Argüelles, M. Bischoff, B. Droste, S. Evans, W. Kröll, J.-L. Lagardère, A. Lina, J. Lumsden, D. Ranque, S. Rasmussen, P. Reutlinger, S. Robins, H. Terho, and A. Wittlöw, European aeronautics: a vision for 2020 - a synopsis, Tech. Rep. 3-4 (Air \& Space Europe, 2001).

[3] ACARE (Institution), Strategic Research and Innovation Agenda, Tech. Rep. June (Advisory Council for Aviation Research and Innovation in Europe, 2016).

[4] K. A. Thole, W. Whitlow, M. J. Benzakein, and S. J. Csonka, National Academies Press (2016) p. 122.

[5] S. W. Ashcraft, A. S. Padron, and K. A. Pascioni, Review of Propulsion Technologies for N+3 Subsonic Vehicle Concepts, Tech. Rep. October (Glenn Research Center, NASA, Cleveland, Ohio, 2011).

[6] C. Pornet and A. T. Isikveren, Conceptual design of hybrid-electric transport aircraft, Progress in Aerospace Sciences 79, 114 (2015).

[7] M. Hepperle, Electric Flight-Potential and Limitations, Tech. Rep. (German Aerospace Center, Braunschweig, 2012).

[8] N. K. Madavan, R. Del Rosario, and A. L. Jankovsky, Hybrid-Electric and Distributed Propulsion Technologies for Large Commercial Air Transports : A NASA Perspective " Advanced Air Transport Technology Project", (2015).

[9] S. Stückl, J. van Toor, and H. Lobentanzer, VOLTAIR - The All Electric Propulsion Concept Platform - A Vision For Atmospheric Friendly Flight, in 28th Congress of the International Council of the Aeronautical Sciences (2012) pp. 1-11.

[10] Rolls-Royce and Airbus Group, E-Thrust - Electrical Distributed Propulsion System Concept for Lower Fuel Consumption, Fewer Emissions and Less Noise, (2014).

[11] M. K. Bradley and C. K. Droney, Subsonic Ultra Green Aircraft Research: Phase I, Tech. Rep. April (2011).

[12] B. Schiltgen, A. R. Gibson, M. Green, and J. Freeman, More Electric Aircraft: "Tube and Wing" Hybrid Electric Distributed Propulsion with Superconducting and Conventional Electric Machines, in SAE Technical Paper 2013-01-2306 (2013).

[13] A. T. Isikveren, A. Seitz, P. C. Vratny, C. Pornet, K. O. Plötner, M. Hornung, and B. L. V, Conceptual studies of universally-electric systems architectures suitable for transport aircraft, in Deutscher Luft- und Raumfahrtkongress 2012, June 2016 (2012) pp. Document-ID: 281368.

[14] IEEE Industry Applications Society. Annual Meeting., Energy Storage Systems for High Power Applications, in 51st Annual Meeting of the IEEE Industry Applications Society (IAS) (Institute of Electrical and Electronics Engineers, 2015).

[15] R. Todd, D. Wu, J. A. dos Santos Girio, M. Poucand, and A. J. Forsyth, Supercapacitor-based energy management for future aircraft systems, in 2010 Twenty-Fifth Annual IEEE Applied Power Electronics Conference and Exposition (APEC) (IEEE, 2010) pp. 1306-1312.

[16] D. Shin, K. Lee, and N. Chang, Fuel economy analysis of fuel cell and supercapacitor hybrid systems, International Journal of Hydrogen Energy 41, 1381 (2016). 
[17] G. Cinar, D. N. Mavris, M. Emeneth, A. Schneegans, and Y. Fefermann, Development of Parametric Power Generation and Distribution Subsystem Models at Conceptual Aircraft Design Stage, in 55th AIAA Aerospace Sciences Meeting (American Institute of Aeronautics and Astronautics, Reston, Virginia, 2017) pp. 1-22.

[18] S. J. Gerssen-Gondelach and A. P. C. Faaij, Performance of batteries for electric vehicles on short and longer term, Journal of Power Sources 212, 111 (2012).

[19] B. Scrosati and J. Garche, Lithium batteries: Status, prospects and future, Journal of Power Sources 195, 2419 (2010), arXiv:97/0302-O23 [0935-9648] .

[20] M. M. Thackeray, C. Wolverton, and E. D. Isaacs, Electrical energy storage for transportation-approaching the limits of, and going beyond, lithium-ion batteries, Energy \& Environmental Science 5, 7854 (2012).

[21] B. Scrosati, J. Hassoun, and Y.-K. Sun, Lithium-ion batteries. A look into the future, Energy \& Environmental Science 4, 3287 (2011).

[22] L. F. Nazar, M. Cuisinier, and Q. Pang, Lithium-sulfur batteries, MRS Bulletin 39, 436 (2014).

[23] Y. Deng, J. Li, T. Li, X. Gao, and C. Yuan, Life cycle assessment of lithium sulfur battery for electric vehicles, Journal of Power Sources 343, 284 (2017).

[24] M. Liu, X. Qin, Y. He, B. Li, and F. Kang, Recent innovative configurations in high-energy lithium-sulfur batteries, Journal of Materials Chemistry A 5, 5222 (2017).

[25] K. G. Gallagher, S. Goebel, T. Greszler, M. Mathias, W. Oelerich, D. Eroglu, and V. Srinivasan, Quantifying the promise of lithium-air batteries for electric vehicles, Energy \& Environmental Science 7, 1555 (2014).

[26] M. Zackrisson, K. Fransson, J. Hildenbrand, G. Lampic, and C. O’Dwyer, Life cycle assessment of lithiumair battery cells, Journal of Cleaner Production 135, 299 (2016).

[27] P. G. Bruce, S. a. Freunberger, L. J. Hardwick, and J.-M. Tarascon, Li-O2 and Li-S batteries with high energy storage, Nature Materials 11, 172 (2011).

[28] M. K. Bradley and C. K. Droney, Subsonic Ultra Green Aircraft Research: Phase II - Volume II - Hybrid Electric Design Exploration, Tech. Rep. (Boeing Research and Technology, Huntington Beach, California, 2011).

[29] C. E. Jones, P. J. Norman, S. J. Galloway, M. J. Armstrong, and A. M. Bollman, Comparison of Candidate Architectures for Future Distributed Propulsion Aircraft, IEEE Transactions on Applied Superconductivity 26 (2016), 10.1109/TASC.2016.2530696.

[30] C. A. Luongo, P. J. Masson, T. Nam, D. Mavris, H. D. Kim, G. V. Brown, M. Waters, and D. Hall, Next generation more-electric aircraft: a potential application for hts superconductors, IEEE Transactions on Applied Superconductivity 19, 1055 (2009).

[31] R. Schiferl, A. Flory, S. Umans, and W. Livoti, High Temperature Superconducting Synchronous Motors: Economic Issues for Industrial Applications, in 2006 Record of Conference Papers - IEEE Industry Applications Society 53rd Annual Petroleum and Chemical Industry Conference, Vol. 15 (2006) pp. 1-9.

[32] K. Sivasubramaniam, T. Zhang, M. Lokhandwalla, E. T. Laskaris, J. W. Bray, B. Gerstler, M. R. Shah, and J. P. Alexander, Development of a high speed HTS generator for airborne applications, in IEEE Transactions on Applied Superconductivity, Vol. 19 (2009) pp. 1656-1661.

[33] C. A. Masson, Philippe J., Luongo, High Power Density Superconducting Motor for All-Electric Aircraft Propulsion, IEEE Transactions on Applied Superconductivity 15, 2226 (2005).

[34] G. Brown, Weights and Efficiencies of Electric Components of a Turboelectric Aircraft Propulsion System, in 49th AIAA Aerospace Sciences Meeting including the New Horizons Forum and Aerospace Exposition, January (2011) pp. 1-18. 
[35] A. Lowe and D. Mavris, Technology Selection for Optimal Power Distribution Efficiency in a Turboelectric Propulsion System, SAE International Journal of Aerospace 5, 425 (2012).

[36] H. J. M. Ter Brake and G. F. M. Wiegerinck, Low-power cryocooler survey, Cryogenics 42, 705 (2002).

[37] H. X. Xi, W. Z. Gong, Y. Zhang, Y. F. Bi, H. K. Ding, H. Wen, B. Hou, and Y. Xin, China's 33.5 m, 35 kV/2 kA HTS ac power cable's operation in power grid, Physica C: Superconductivity and its Applications 448, 1054 (2006).

[38] P. J. Masson, G. V. Brown, D. S. Soban, and C. A. Luongo, HTS machines as enabling technology for allelectric airborne vehicles, Superconductor Science and Technology 20, 748 (2007).

[39] K. Petermaier, Electric propulsion components with high power densities for aviation, (2015).

[40] Siemens AG, World-record electric motor for aircraft, (2015).

[41] H. Kuhn, A. Seitz, L. Lorenz, A. Isikveren, and A. Sizmann, Progress and perspectives of electric air transport, in 28th Congress of the International Council of the Aeronautical Sciences 2012, ICAS 2012, Vol. 6 (2012).

[42] L. Lorenz, A. Seitz, H. Kuhn, and A. Sizmann, Hybrid Power Trains for Future Mobility, in Deutscher Luftund Raumfahrtkongress (2013) pp. 1-17, arXiv:301316.

[43] M. Hornung, A. T. Isikveren, M. Cole, and A. Sizmann, Ce-Liner - Case Study for eMobility in Air Transportation, in 2013 Aviation Technology, Integration, and Operations Conference (American Institute of Aeronautics and Astronautics, Reston, Virginia, 2013) pp. 1-11.

[44] J. Felder, H. Kim, and G. Brown, Turboelectric Distributed Propulsion Engine Cycle Analysis for HybridWing-Body Aircraft, in 47th AIAA Aerospace Sciences Meeting including The New Horizons Forum and Aerospace Exposition, January (2009) pp. AIAA 2009-1132.

[45] J. Y. Hung and L. F. Gonzalez, On parallel hybrid-electric propulsion system for unmanned aerial vehicles, Progress in Aerospace Sciences 51, 1 (2012).

[46] R. Jagannath, S. Bane, M. R. Nalim, and J. Khan, A Simplified Method to Calculate the Fuel Burn of a Hybrid-Electric Airplane, in 50th AIAA/ASME/SAE/ASEE Joint Propulsion Conference (AIAA Propulsion and Energy Forum, 2014) pp. 1-11.

[47] C. Friedrich and P. A. Robertson, Hybrid-electric propulsion for automotive and aviation applications, CEAS Aeronautical Journal 6, 279 (2015).

[48] K. T. Chau and Y. S. Wong, Overview of power management in hybrid electric vehicles, Energy Conversion and Management 43, 1953 (2002).

[49] C. C. Chan, A. Bouscayrol, and K. Chen, Electric, hybrid, and fuel-cell vehicles: Architectures and modeling, IEEE Transactions on Vehicular Technology 59, 589 (2010).

[50] C. Pornet, S. Kaiser, A. Isikveren, and M. Hornung, Integrated fuel-battery hybrid for a narrow-body sized transport aircraft, Aircraft Engineering and Aerospace Technology 86, 568 (2014).

[51] G. Cinar, D. N. Mavris, M. Emeneth, A. Schneegans, C. Riediger, Y. Fefermann, and A. Isikveren, Sizing, Integration and Performance Evaluation of Hybrid Electric Propulsion Subsystem Architectures, in 55th AIAA Aerospace Sciences Meeting, January (2017) pp. 1-22.

[52] T. Nam, D. Soban, and D. Mavris, Power Based Sizing Method for Aircraft Consuming Unconventional Energy, 43rd AIAA Aerospace Sciences Meeting and Exhibit, 1 (2005).

[53] C. Pornet, C. Gologan, P. C. Vratny, A. Seitz, O. Schmitz, A. T. Isikveren, and M. Hornung, Methodology for Sizing and Performance Assessment of Hybrid Energy Aircraft, Journal of Aircraft 52, 341 (2015).

[54] K. R. Antcliff, M. D. Guynn, T. Marien, D. P. Wells, S. J. Schneider, and M. J. Tong, Mission Analysis and Aircraft Sizing of a Hybrid-Electric Regional Aircraft, in 54th AIAA Aerospace Sciences Meeting (2016). 
[55] A. Isikveren, S. Kaiser, C. Pornet, and P. Vratny, Pre-design strategies and sizing techniques for dual-energy aircraft, Aircraft Engineering and Aerospace Technology 86, 525 (2014).

[56] Y. Fefermann, C. Maury, C. Level, K. Zarati, J.-P. Salanne, C. Pornet, B. Thoraval, and A. T. Isikveren, Hybrid-Electric Motive Power Systems for Commuter Transport Applications, 30th Congress of the International Council of the Aeronautical Sciences , 1 (2016).

[57] J. Roskam, Airplane Design (DARcorporation, 1985) p. 2615.

[58] N. K. Borer, M. D. Patterson, J. K. Viken, M. D. Moore, J. Bevirt, A. M. Stoll, and A. R. Gibson, Design and Performance of the NASA SCEPTOR Distributed Electric Propulsion Flight Demonstrator, in 16th AIAA Aviation Technology, Integration, and Operations Conference (2016).

[59] A. M. Stoll and G. Veble Mikic, Design Studies of Thin-Haul Commuter Aircraft with Distributed Electric Propulsion, in 16th AIAA Aviation Technology, Integration, and Operations Conference (2016).

[60] R. de Vries, M. T. Brown, and R. Vos, A Preliminary Sizing Method for Hybrid-Electric Aircraft Including Aero-Propulsive Interaction Effects, in 2018 Aviation Technology, Integration, and Operations Conference (American Institute of Aeronautics and Astronautics, Reston, Virginia, 2018).

[61] D. Howe, Aircraft conceptual design synthesis, Professional Engineering (2000), 10.1002/9781118352700, arXiv:arXiv:1011.1669v3 .

[62] E. Torenbeek, Synthesis of subsonic airplane design (Delft University Press, Delft, 1979) arXiv:arXiv:1011.1669v3 .

[63] G. V. Brown, A. F. Kascak, B. Ebihara, D. Johnson, B. Choi, M. Siebert, and C. Buccieri, NASA Glenn Research Center Program in High Power Density Motors for Aeropropulsion, (2005).

[64] S. Stückl, Methods for the Design and Evaluation of Future Aircraft Concepts Utilizing Electric Propulsion Systems, Phd dissertation, Munich Technical University (2016).

[65] N. E. Anderson, S. H. Loewenthal, and J. D. Black, An Analytical Method To Predict Efficiency of Aircraft Gearboxes, Nasa Tm-83716, Usaavscom Tr 84-C-8 (1984), 10.1115/1.3258750.

[66] D. P. Raymer, Aircraft design: A conceptual approach (AIAA Education Series, 2012).

[67] J. Larminie, J. Lowry, and J. Wiley, Electric Vehicle Technology Explained, Tech. Rep.

[68] R. A. McDonald, Electric Propulsion Modeling for Conceptual Aircraft Design, in 52nd Aerospace Sciences Meeting (2014).

[69] T. J. Haugan, J. D. Long, L. A. Hampton, and P. N. Barnes, Design of compact, lightweight power transmission devices for specialized high power applications, SAE International Journal of Aerospace 1, 1088 (2009).

[70] G. Brown, Materials Aspects of Turboelectric Aircraft Propulsion, (2009).

[71] M. Gouge, J. Demko, B. McConnell, and O. J. M. Pfotenhauer, Cryogenics Assessment Report, Oak Ridge National Laboratory USA, 1 (2002).

[72] A. Seitz, A. T. Isikveren, and M. Hornung, Pre-Concept Performance Investigation of Electrically Powered Aero-Propulsion Systems, 49th AIAA/ASME/SAE/ASEE Joint Propulsion Conference , 1 (2013).

[73] W. Tahil, How Much Lithium does a LiIon EV battery really need ? Meridian , 1 (2010).

[74] P. C. Vratny, C. Pornet, C. Gologan, A. T. Isikveren, and M. Hornung, Battery Pack Modeling and Optimization Within Universall-Electric Aircraft, in Ceas 2013 (2013).

[75] M. Chen and G. A. Rincón-Mora, Accurate electrical battery model capable of predicting runtime and I-V performance, IEEE Transactions on Energy Conversion 21, 504 (2006).

[76] A. Seitz, O. Schmitz, A. T. Isikveren, and M. Hornung, Electrically Powered Propulsion: Comparison and Contrast to Gas Turbines, Deutscher Luft- und Raumfahrtkongress 2012, 1 (2012). 
[77] A. S. Gohardani, A synergistic glance at the prospects of distributed propulsion technology and the electric aircraft concept for future unmanned air vehicles and commercial/military aviation, Progress in Aerospace Sciences 57, 25 (2013).

[78] L. Mirande and J. Brennan, Aerodynamic effects of wingtip-mounted propellers and turbines, in 4th Applied Aerodynamics Conference (1986).

[79] J. Thauvin, G. Barraud, M. Budinger, X. Roboam, D. Leray, and B. Sareni, Hybrid Regional Aircraft: A Comparative Review of New Potentials Enabled by Electric Power, in 52nd AIAA/SAE/ASEE Joint Propulsion Conference (2016).

[80] G. J. J. Ruijgrok, Elements of airplane performance (Delft University Press, 2009) p. 378.

[81] ICAO Annex 16 - Environmental Protection, Volume II - Aircraft Engine Emissions, Tech. Rep. (International Civil Aviation Organization, 2008).

[82] H. Mulder, AE4462 Aircraft Emissions and Climate Effects, (2018).

[83] ICAO Engine Exhaust Emissions Data Bank, (2018).

[84] I. P. O. C. Change, Climate change: the IPCC scientific assessment, Mass, Cambridge , 364 (1990).

[85] J. S. Fuglestvedt, I. S. Isaksen, and W. C. Wang, Estimates of indirect global warming potentials for CH4, CO and NOx, Climatic Change 34, 405 (1996).

[86] F. Svensson, A. Hasselrot, and J. Moldanova, Reduced environmental impact by lowered cruise altitute for liquid hydrogen-fuelled aircraft, Aerospace Science and Technology 8, 307 (2004).

[87] J. Hoelzen, Y. Liu, B. Bensmann, C. Winnefeld, A. Elham, J. Friedrichs, and R. Hanke-Rauschenbach, Conceptual design of operation strategies for hybrid electric aircraft, Energies 11, 1 (2018).

[88] W.-C. Wang, L. Tao, J. Markham, Y. Zhang, E. Tan, L. Batan, M. Biddy, W.-C. Wang, L. Tao, Y. Zhang, E. Tan, E. Warner, and M. Biddy, Review of Biojet Fuel Conversion Technologies, www.nrel.gov/publications , 98 (2016).

[89] C. E. Till, Y. I. L. Chang, K. Sridharan, Seai, C. Mcmillan, R. Boardman, M. Mckellar, P. Sabharwall, M. Ruth, S. Bragg-sitton, C. Mcmillan, R. Boardman, M. Mckellar, P. Sabharwall, M. Ruth, S. Bragg-sitton, A. C. Kadak, S. A. Effective, N.-1. W. Reactor, M. Readiness, M. Ding, J. L. Kloosterman, T. Kooijman, R. Linssen, T. Abram, B. Marsden, T. Wickham, J. Conca, S. Bureau, and W. E. Balances, Recommended citation : NREL ( National Renewable Energy Laboratory ). 2016 . 2016 Annual Technology Baseline . Golden , CO : National Renewable Energy Laboratory. International Energy Agency 812, 86 (2016).

[90] A. Filippone, Advanced Aircraft Flight Performance (Cambridge University Press, Cambridge, 2012).

[91] A. Filippone and Z. Mohamed-Kassim, Multi-disciplinary simulation of propeller-turboprop aircraft flight, Aeronautical Journal 116, 985 (2012).

[92] P. Della Vecchia, Development of Methodologies for the Aerodynamic Design and Optimization of New Regional Turboprop Aircraft, Tech. Rep. (2014).

[93] D. Verstraete, A. Gong, D. D.-C. Lu, and J. L. Palmer, Experimental investigation of the role of the battery in the AeroStack hybrid, fuel-cell-based propulsion system for small unmanned aircraft systems, International Journal of Hydrogen Energy 40, 1598 (2015).

[94] R. Jansen, C. Bowman, A. Jankovsky, R. Dyson, and J. Felder, Overview of NASA Electrified Aircraft Propulsion (EAP) Research for Large Subsonic Transports, 53rd AIAA/SAE/ASEE Joint Propulsion Conference , 1 (2017).

[95] M. Strack, G. Pinho Chiozzotto, M. Iwanizki, M. Plohr, and M. Kuhn, Conceptual Design Assessment of Advanced Hybrid Electric Turboprop Aircraft Configurations, in 17th AIAA Aviation Technology, Integration, and Operations Conference (2017). 
[96] M. H. Snyder, Jr. and G. W. Zumwalt, Effects of wingtip-mounted propellers on wing lift and induced drag. Journal of Aircraft 6, 392 (1969).

[97] J. C. Patterson and G. R. Bartlett, Evaluation of installed performance of a wing-tip-mounted pusher turboprop on a semispan wing, NASA Technical paper (1987).

[98] A. M. Stoll, J. Bevirt, M. D. Moore, W. J. Fredericks, and N. K. Borer, Drag Reduction Through Distributed Electric Propulsion, in 14th AIAA Aviation Technology, Integration, and Operations Conference (2014) pp. 16-20.

[99] M. Hepperle, Aspects of Distributed Propulsion - A View on Regional Aircraft, (2016). 


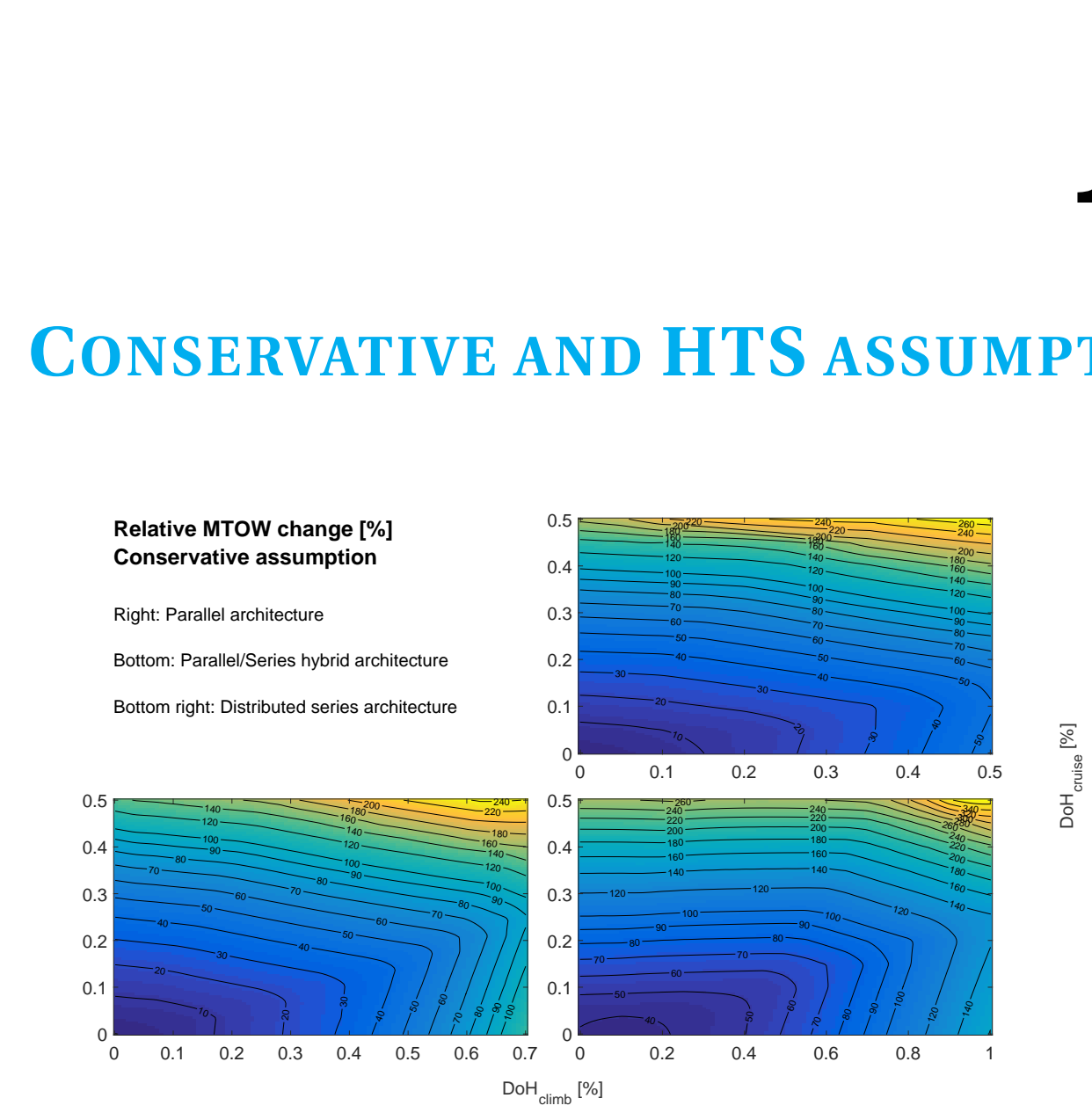

A

Relative MTOW change [\%]

Right: Parallel architecture

Bottom: Parallel/Series hybrid architecture

Bottom right: Distributed series architecture

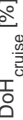

Figure A.1: MTOM response surface conservative assumption.

Relative MTOW change [\%]

HTS technology assumption

Right: Parallel architecture

Bottom: Parallel/Series hybrid architecture

Bottom right: Distributed series architecture
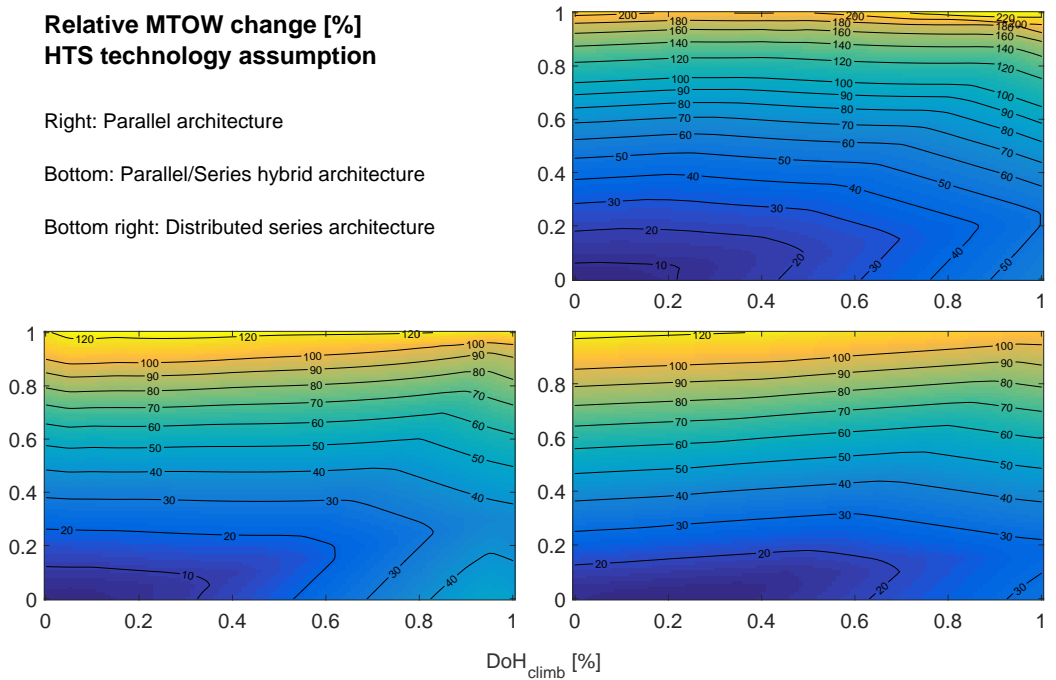

$\frac{0}{\circ}$
$\frac{0}{3}$
$\frac{.0}{0}$
$\frac{0}{\circ}$

Figure A.2: MTOM response surface futuristic assumption. 
Electrical Energy surplus [\%]

Conservative assumption

Right: Parallel architecture

Bottom: Parallel/Series hybrid architecture

Bottom right: Distributed series architecture
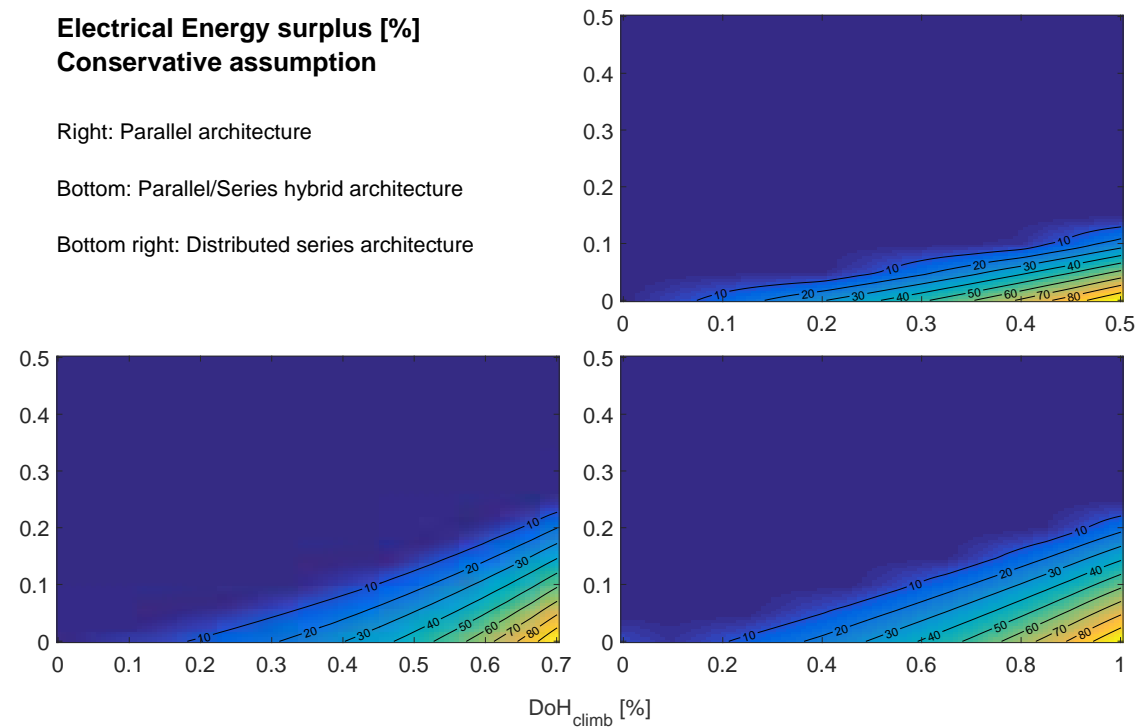

Figure A.3: Battery sizing requirements response surface conservative assumption.

Electrical Energy surplus [\%]

HTS technology assumption

Right: Parallel architecture

Bottom: Parallel/Series hybrid architecture

Bottom right: Distributed series architecture
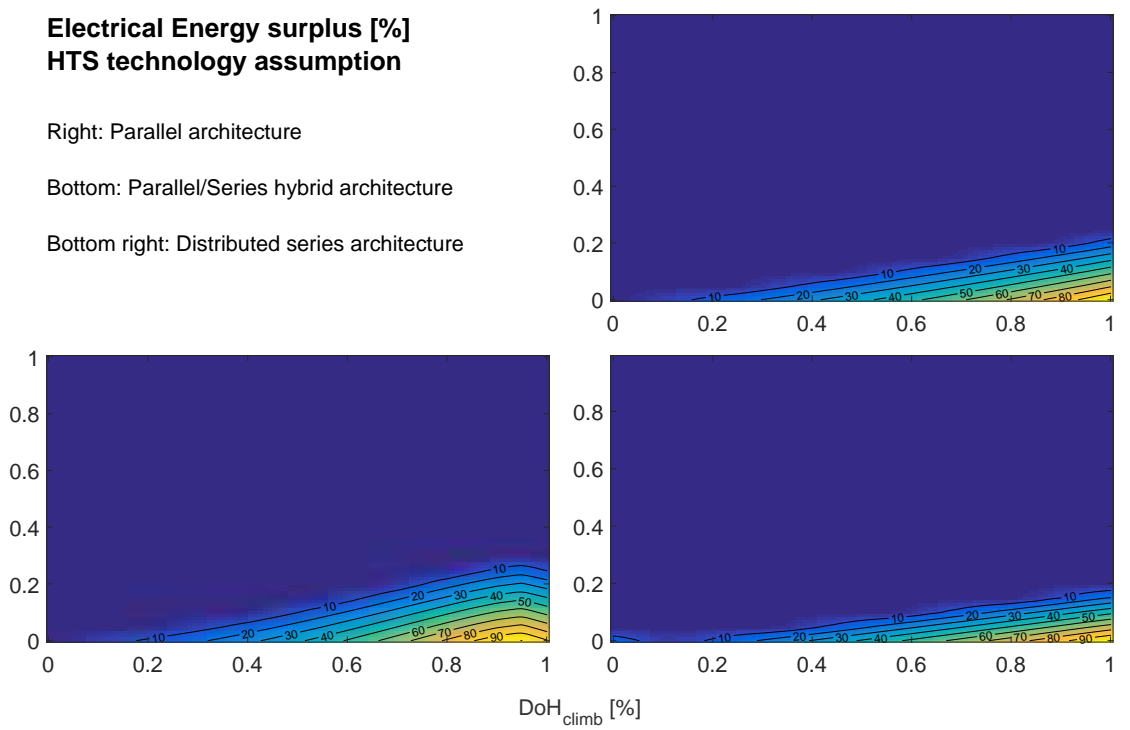

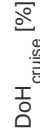

Figure A.4: Battery sizing requirements response surface futuristic assumption. 
Relative fuel mass change [\%]

Conservative assumption

Right: Parallel architecture

Bottom: Parallel/Series hybrid architecture

Bottom right: Distributed series architecture
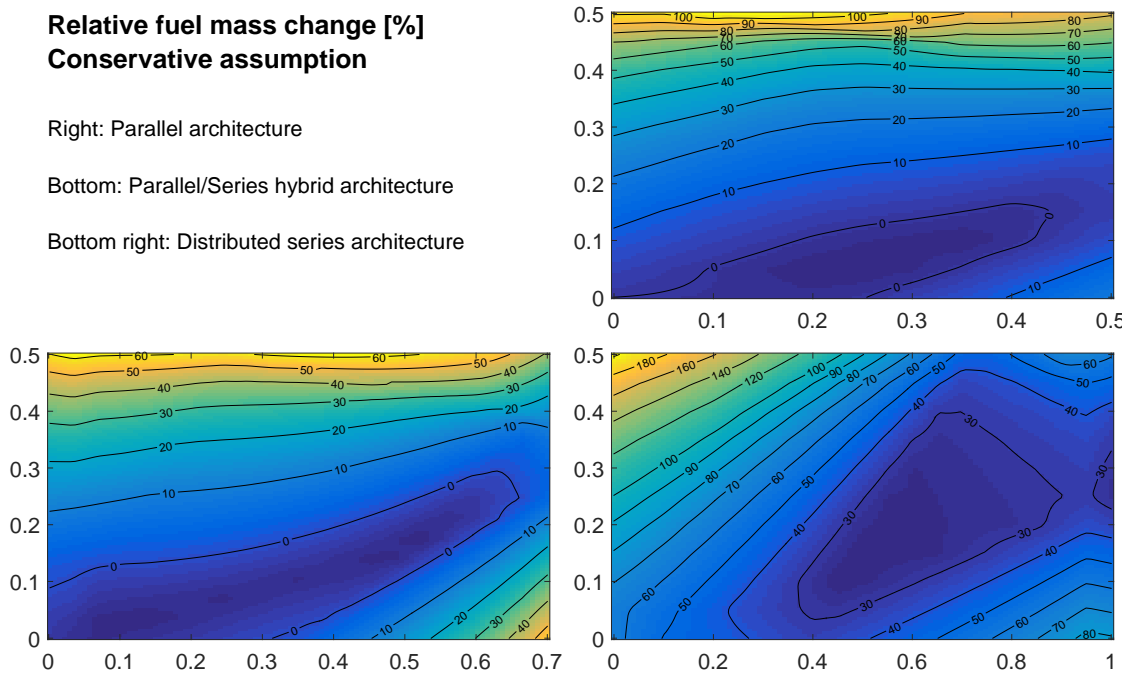

Figure A.5: Fuel response surface conservative assumption.

Relative fuel mass change [\%]

HTS technology assumption

Right: Parallel architecture

Bottom: Parallel/Series hybrid architecture

Bottom right: Distributed series architecture
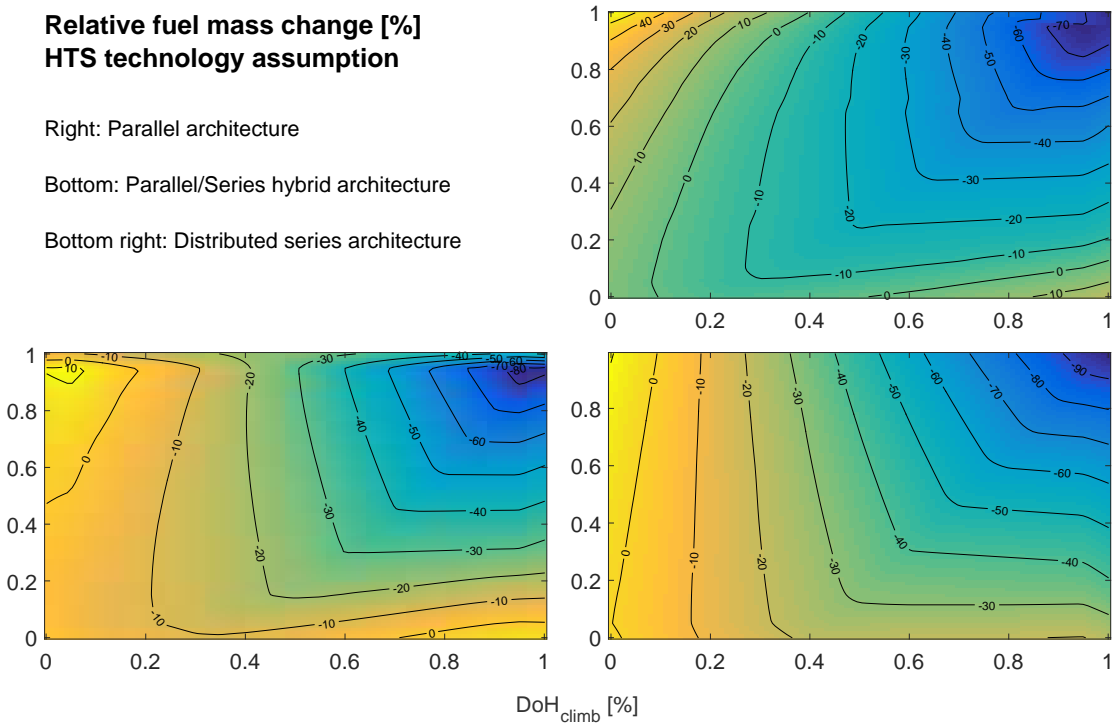

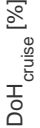

Figure A.6: Fuel response surface futuristic assumption. 
Relative Energy change [\%]

Conservative assumption

Right: Parallel architecture

Bottom: Parallel/Series hybrid architecture

Bottom right: Distributed series architecture
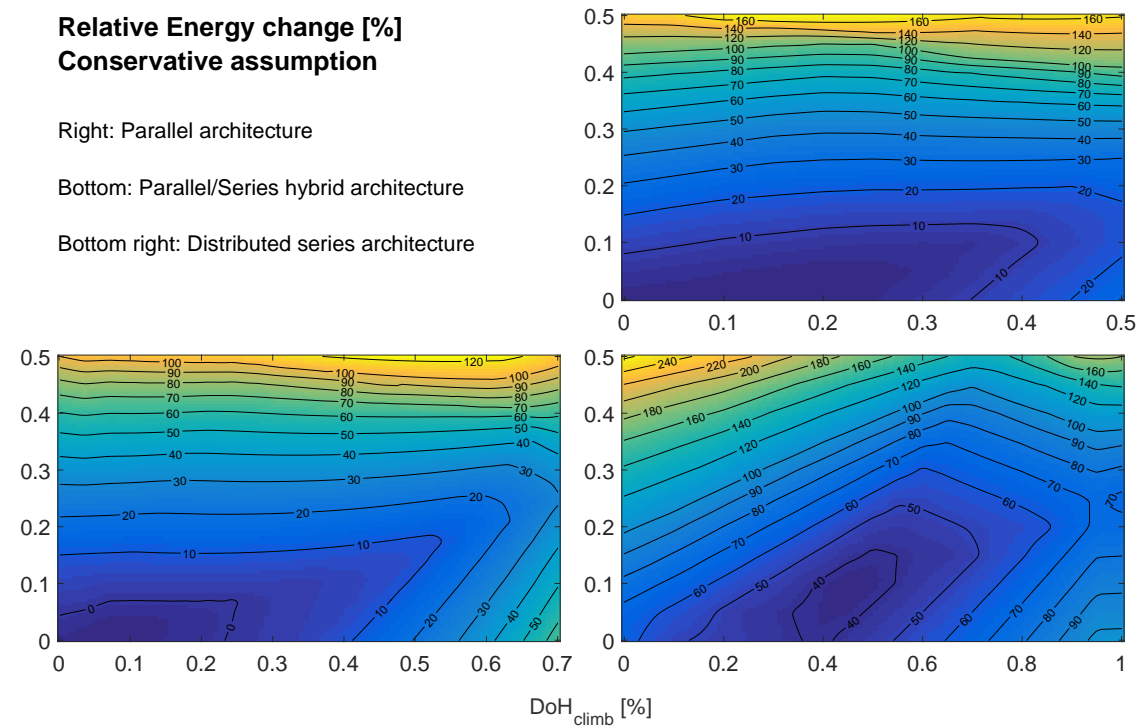

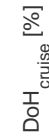

Figure A.7: Total energy response surface conservative assumption.

Relative Energy change [\%]

HTS technology assumption

Right: Parallel architecture

Bottom: Parallel/Series hybrid architecture

Bottom right: Distributed series architecture
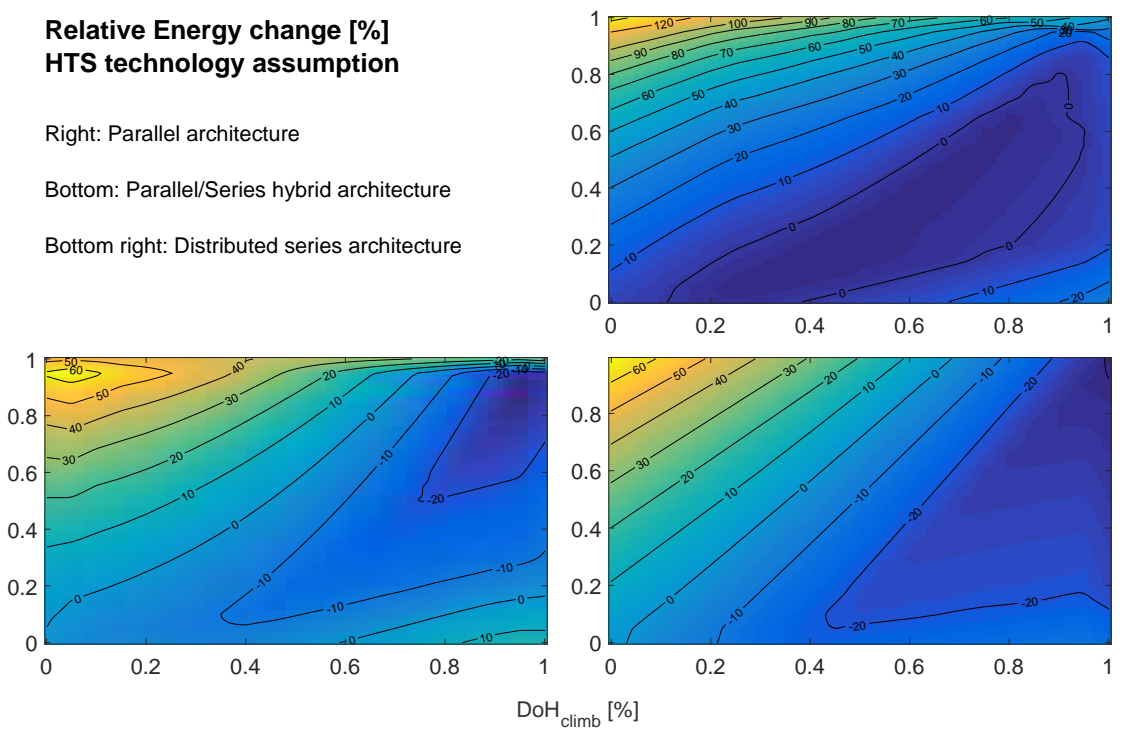

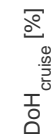

Figure A.8: Total energy response surface futuristic assumption. 
HEP overall efficiency change [\%] Conservative assumption

Right: Parallel architecture

Bottom: Parallel/Series hybrid architecture

Bottom right: Distributed series architecture
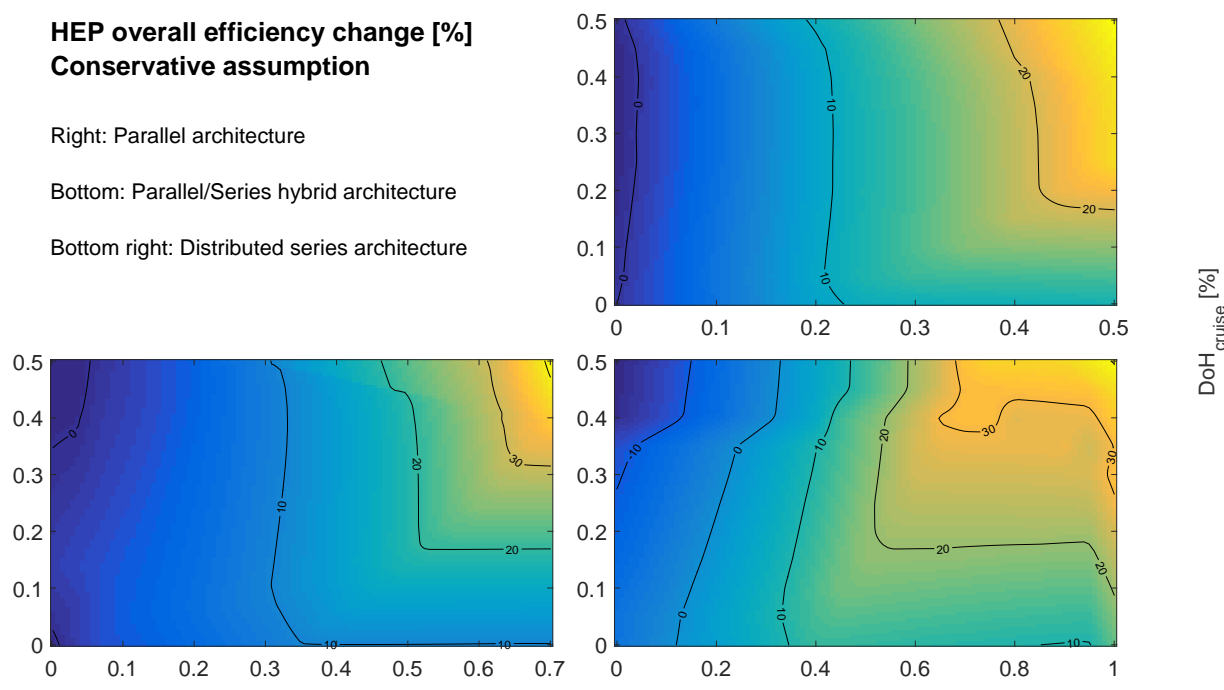

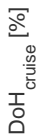

Figure A.9: System efficiency response surface conservative assumption.

HEP overall efficiency change [\%]

HTS technology assumption

Right: Parallel architecture

Bottom: Parallel/Series hybrid architecture

Bottom right: Distributed series architecture
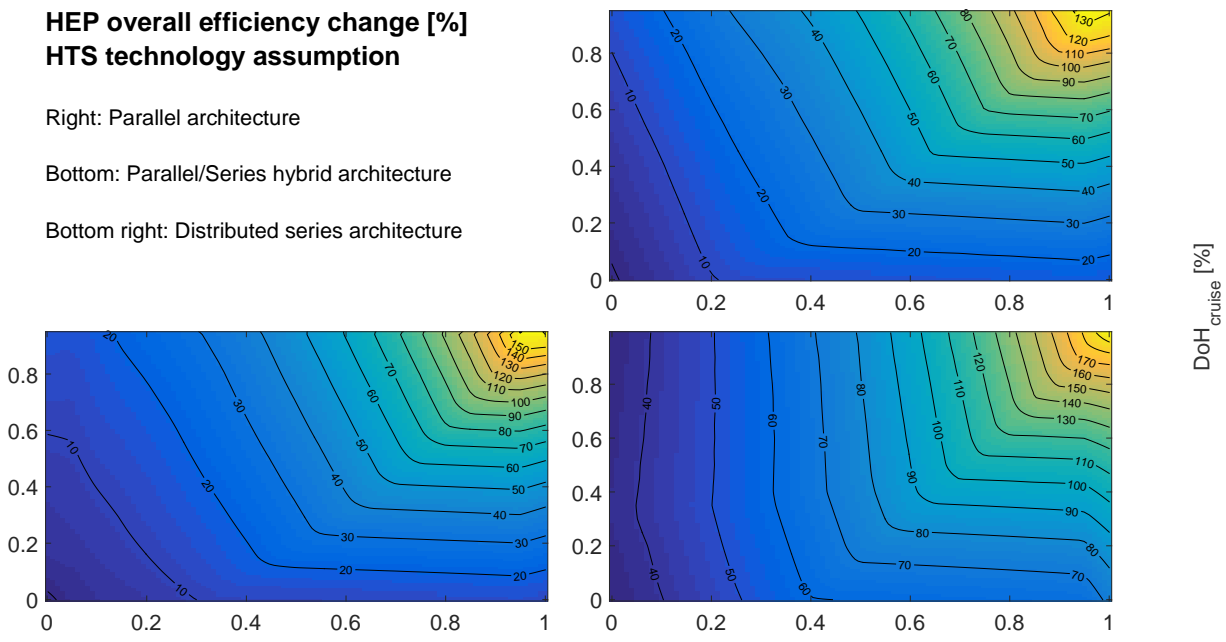

Figure A.10: System efficiency response surface futuristic assumption. 
Relative ESAR change [\%]

Conservative assumption

Right: Parallel architecture

Bottom: Parallel/Series hybrid architecture

Bottom right: Distributed series architecture
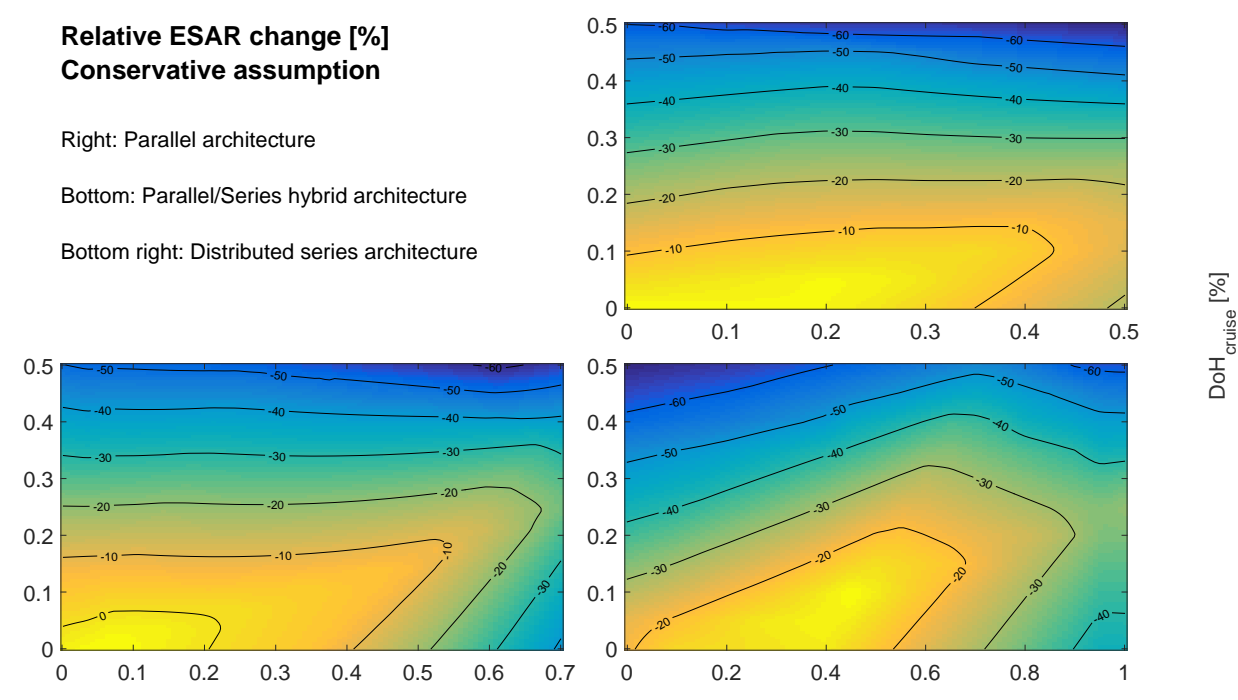

Figure A.1 1: ESAR response surface conservative assumption.

Relative ESAR change [\%]

Conservative assumption

Right: Parallel architecture

Bottom: Parallel/Series hybrid architecture

Bottom right: Distributed series architecture

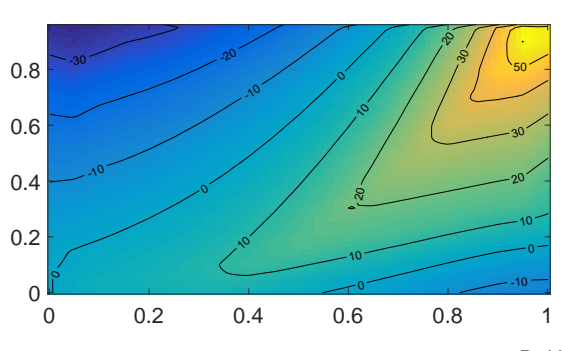

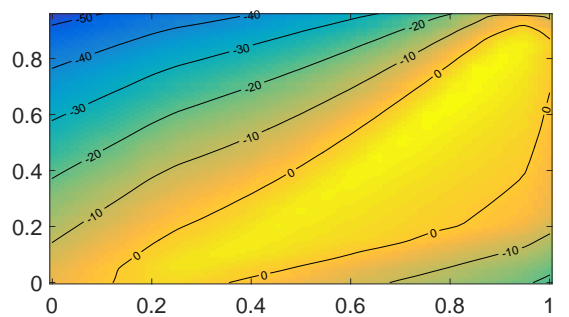

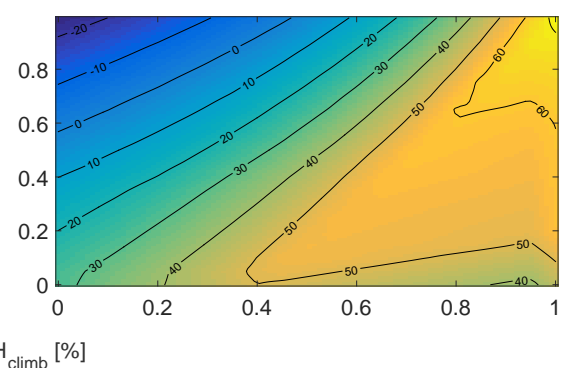

Figure A.12: ESAR response surface futuristic assumption. 
Relative Emissions change [\%]

Conservative assumption

Right: Parallel architecture

Bottom: Parallel/Series hybrid architecture

Bottom right: Distributed series architecture
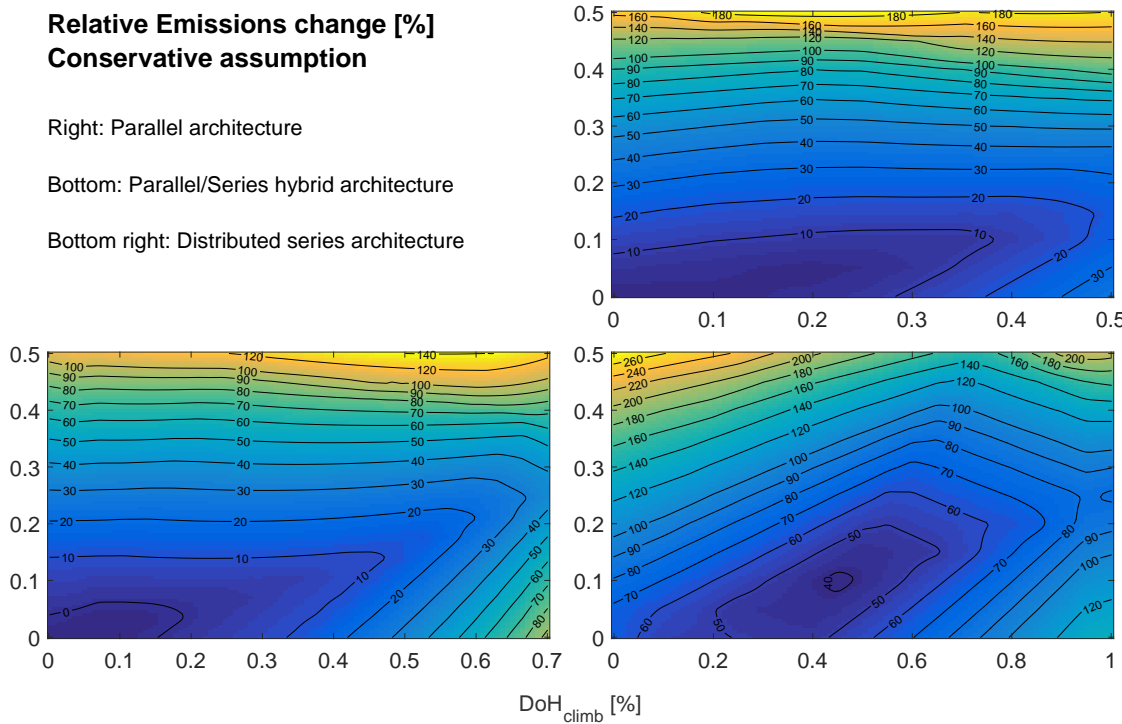

Figure A.13: Emissions response surface conservative assumption.

Relative Emissions change [\%] HTS technology assumption

Right: Parallel architecture

Bottom: Parallel/Series hybrid architecture

Bottom right: Distributed series architecture
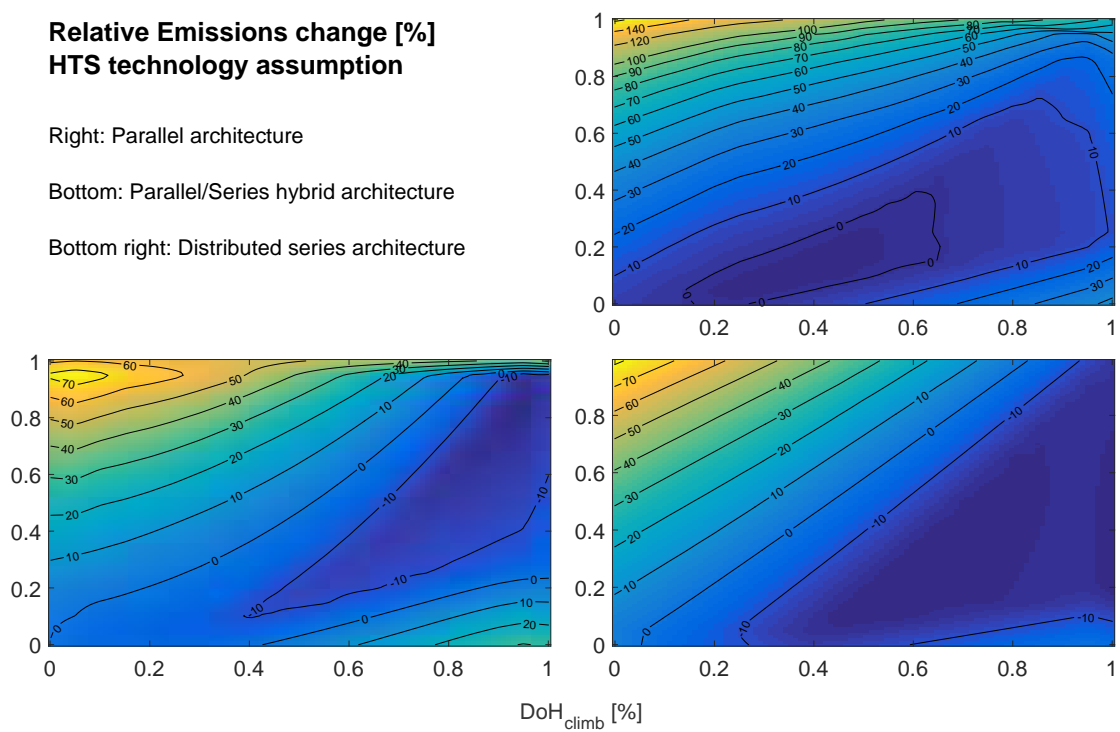

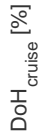

Figure A.14: Emissions response surface futuristic assumption. 



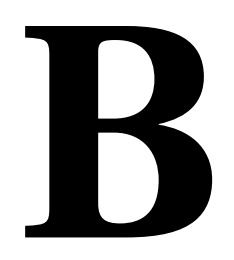

Hybrid ElECTRIC Aircraft SCHEMATICS 


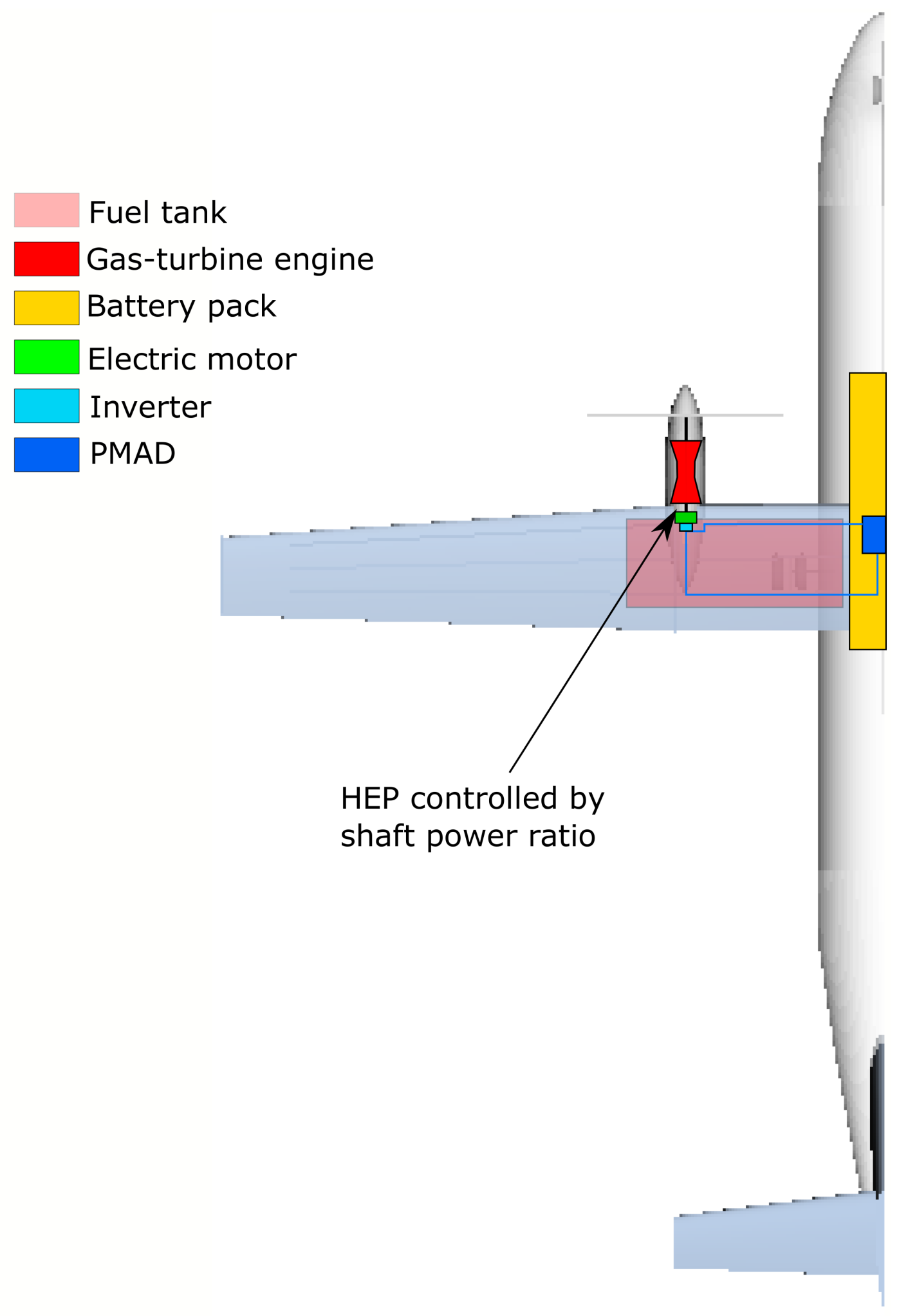

Figure B.1: Parallel architecture schematic. 


\section{Fuel tank}

Gas-turbine engine Battery pack

Electric motor

Inverter

PMAD

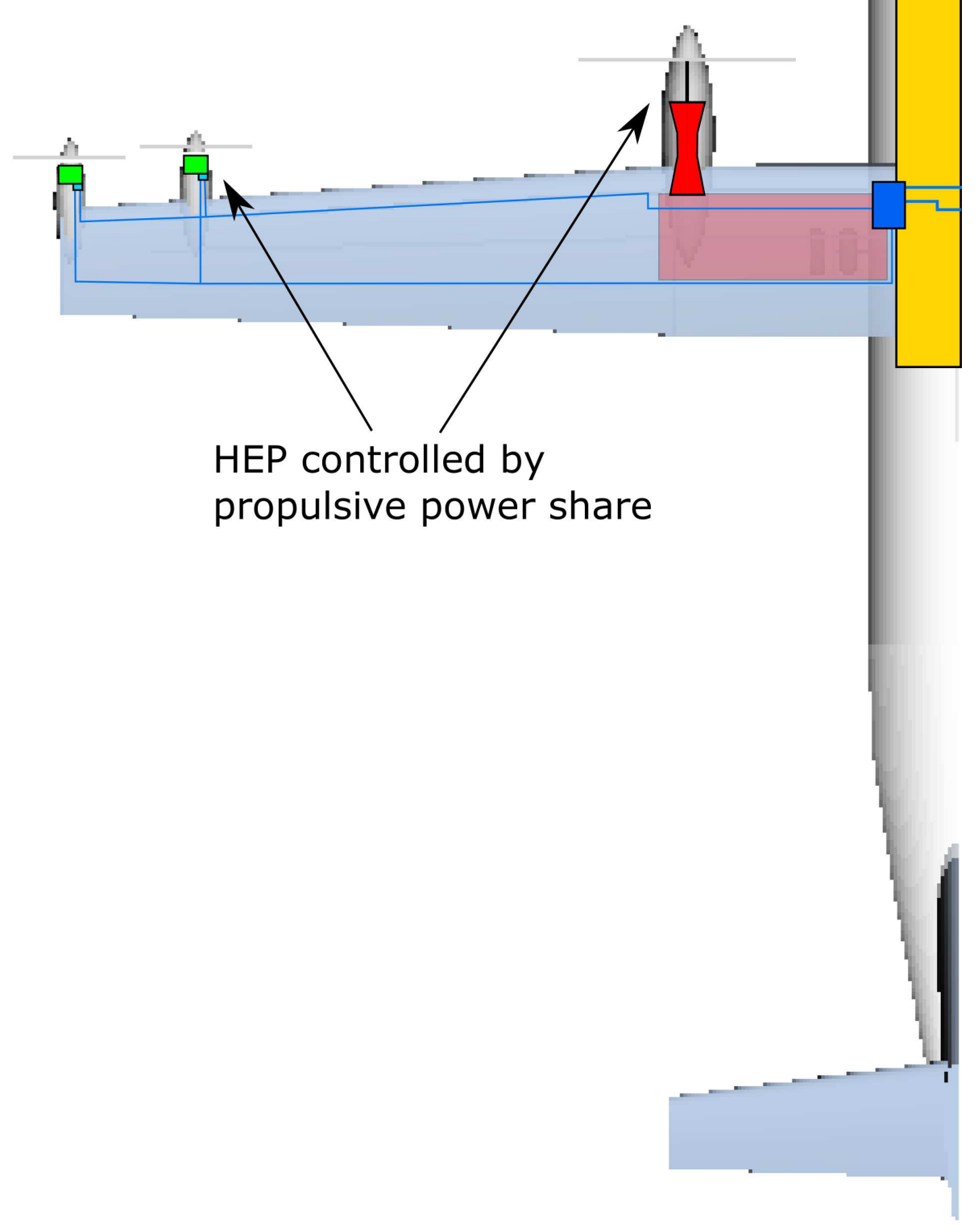

Figure B.2: Series/parallel architecture schematic. 


\section{Fuel tank}

Gas-turbine engine

Battery pack

Electric motor

\section{Inverter}

PMAD

Electric generator

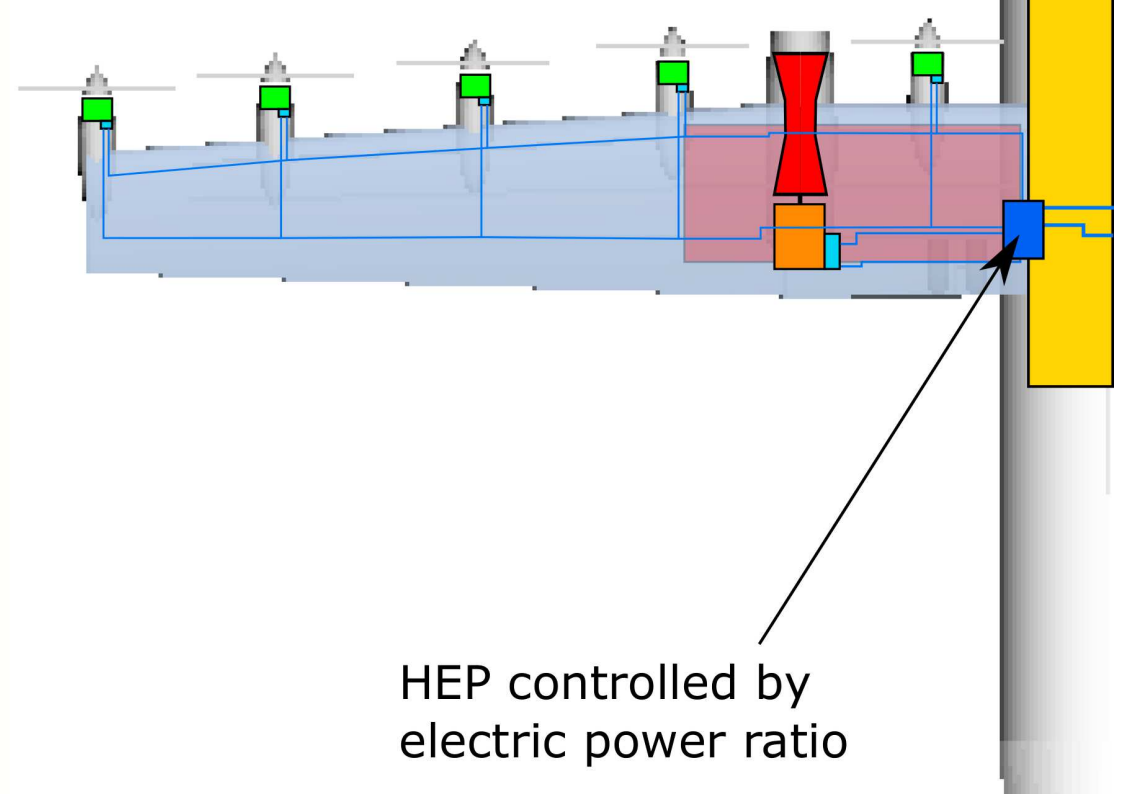

Figure B.3: Series architecture schematic. 